\title{
Compilation of Data on Radionuclide Data for Specific Activity, Specific Heat andFission Product Yields
}

by

B. Boggs (Contact)

Westinghouse Savannah River Company

Savannah River Site

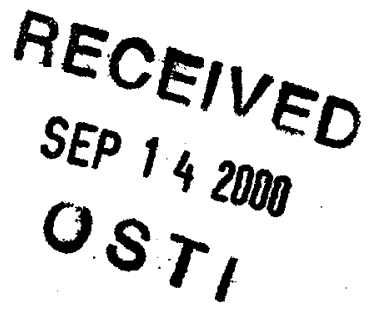

Aiken, South Carolina 29808

A. Gibbs

R. S. Thomason

This paper was prepared in connection with work done under the above contract number with the U. S. Department of Energy. By acceptance of this paper, the publisher and/or recipient acknowledges the U.S. Government's right to retain a nonexclusive, royalty-free license in and to any copyright covering this paper, along with the right to reproduce and to authorize others to reproduce all or part of the copyrighted paper. 


\section{DISCLAIMER}

This report was prepared as an account of work sponsored by an agency of the United States Government. Neither the United States Government nor any agency thereof, nor any of their employees, makes any warranty, express or implied, or assumes any legal liability or responsibility for the accuracy, completeness, or usefulness of any information, apparatus, product or process disclosed, or represents that its use would not infringe privately owned rights. Reference herein to any specific commercial product, process or service by trade name, trademark, manufacturer, or otherwise does not necessarily constitute or imply its endorsement, recommendation, or favoring by the United States Government or any agency thereof. The views and opinions of authors expressed herein do not necessarily state or reflect those of the United States Government or any agency thereof.

This report has been reproduced directly from the best available copy.

Available for sale to the public, in paper, from: U.S. Department of Commerce, National Technical Information Service, 5285 Port Royal Road, Springfield, VA 22161

phone: (800) 553-6847

fax: (703) 605-6900

email: orders@ntis.fedworld.gov

online ordering: http://www.ntis.gov/ordering.htm

Available electronically at http://www.doe.gov/bridge

Available for a processing fee to U.S. Department of Energy and its contractors, in paper, from: U.S. Department of Energy, Office of Scientific and Technical Information, P.O. Box 62, Oak Ridge, TN 37831-0062

phone: (865)576-8401

fax: $(\mathbf{8 6 5}) 576-5728$

email: reports@adonis.osti.gov 


\section{DISCLAIMER}

Portions of this document may be illegible in electronic image products. Images are produced from the best available original document. 
WSRC-TR-99-00344

October, 1999

COMPILATION OF RECENT RADIONUCLIDE DATA FOR SPECIFIC ACTIVITY, SPECIFIC HEAT AND FISSION PRODUCT YIELDS

compiled by

Ann Gibbs

reviewed by

R. S. Thomason 
WSRC-TR-99-00344

October, 1999

COMPILATION OF RECENT RADIONUCLIDE DATA FOR SPECIFIC ACTIVITY, SPECIFIC HEAT AND FISSION PRODUCT YIELDS

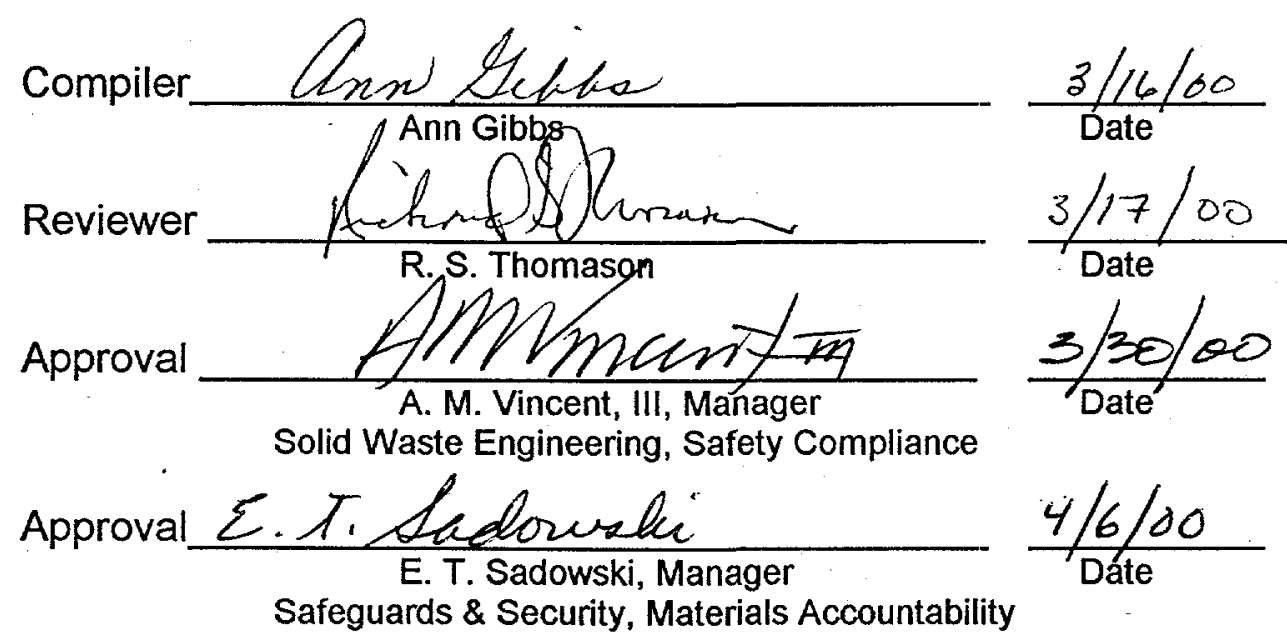


WSRC-TR-99-00344

October, 1999

page iii

\section{Table of Contents}

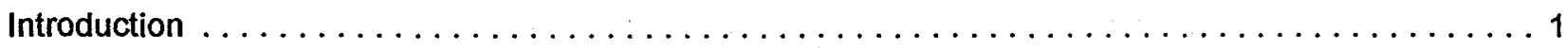

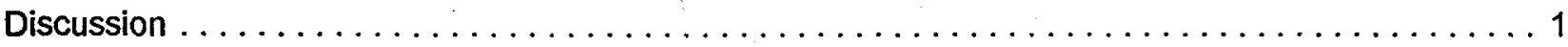

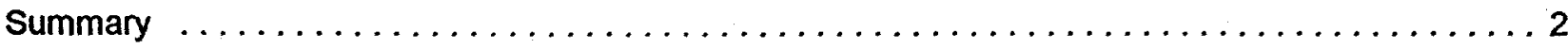

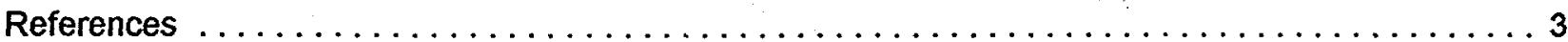




\section{Introduction}

This compilation was undertaken to update the data used in calculation of curie and heat loadings of waste containers in the Solid Waste Management Facility. The data has broad general use and has been cross-checked extensively in order to be of use in the Materials Accountability arena. The fission product cross-sections have been included because they are of use in the Environmental Remediation and Waste Management areas where radionuclides which are not readily detectable need to be calculated from the relative fission yields and material dispersion data.

This document was reviewed and cross-checked by R. B. Thomason in the following manner:

Half lives (seconds): Each value was reviewed in comparison to Reference 6 or Reference 7. Reference 6 was available in an Access ${ }^{\circ}$ database, and that was converted to Excel ${ }^{\circ}$ Spreadsheet format and aligned row wise with the data to be reviewed. A spreadsheet formula was used to compare the report half life and the reference 6 half life for each row that had data from both sources, and to flag the row if the two values differed by more than four percent. The flagged half lives and those that had no corresponding value from reference 6 were compared with half lives from reference 7. Those rows that did not agree with reference 7 , or for which reference 7 had no value, were flagged for further verification by the author.

Half lives (other units): These values were entered directly, and their unit (second, minute, hour, day, year) was entered in an adjacent column. A formula based on the unit was used to calculate each half life in units of seconds, and that value was validated as per the above. The review consisted of validating the formula.

Specific activities and inverses: The formula for calculating the specific activity was compared with a formula known to be correct, and representative specific activities were calculated using the two formulas and shown to be equal. The inverse of the specific activity was calculated by formula, the inverse of the specific activity cell.

Atomic weights: The atomic weight was compared with the atomic number for each radionuclide and flagged if the two numbers differed by more than 0.2 units. This review would have identified gross typographical errors, but it would not have caught errors in the second or higher decimal positions.

Watts/Ci: Not reviewed by RST.

Fission product yields: Not reviewed by RST.

This is the complete set of radionuclides existing in verified data files as of March 15, 2000. There are subsets available, at request from the compiler, for radionuclides which have been produced or brought to SRS and for radionuclides with half-lives greater than whatever breakpoint needed.

\section{Discussion}

There are continual changes in nuclear data which occur due to more exact measurement of half-lives and atomic masses. These changes have not been reflected in the tables used at SRS in Materials Accountability for some 14 years. These tables are used throughout the site to report waste disposal and 
transportation and environmental concentrations of radioisotopes. These quantities are reported in the public media. While the differences between present values and those used 14 years ago are not great, it is important in dealing with the public to present the best data available. Since the data are available, this compilation was made to update SRS tables.

The original version of this compilation was made in 1991-92 to prove that the number of radioisotopes that need to be considered for performance assessments of waste disposal is not in the thousands. The complete compilation has a field for whether or not the radioisotope could have been produced or used in research at SRS. This field has not been included in the printout, although it has been continued in the spreadsheet.

The information for this compilation comes from the referenced sources, most of which have assembled their data from the ENSDF (Evaluated Nuclear Structure Data File) tabulation maintained at the Brookhaven National Laboratory in the United States. Values used in this compilation were originally taken from the 1996 Edition of the Table of Isotopes, but were checked with the latest Internet sources during the June-August, 1999 time period and confirmed in March, 2000 after the review by RST.

The half-lives were converted to seconds to simplify the calculations of specific activities to one formula. Half-lives reported in years are sidereal years so the conversion to days used is 365.26. A sidereal year is the time required for one complete revolution of the earth about the sun, relative to the fixed stars. It is equal to 365 days, 6 hours, 9 minutes, 9.54 seconds ( 365.25636 days) in calendar time units.

The formula for calculation of the specific activity is:

$\mathrm{Ci} / \mathrm{g}=(6.0221 \mathrm{E}+23) /(3.7 \mathrm{E}+10)($ Atomic Mass $)(\lambda)$

where:

$\lambda=\ln 2 /$ half-life in seconds $\quad$ units $=\left(\mathrm{sec}^{-1}\right)$

$6.0221 \mathrm{E}+23=$ Avogadro's constant units $=\left(\mathrm{mol}^{-1}\right)$

$\left\{\right.$ NIST CODATA File Value, $\left.1999=6.02214199 \mathrm{E}+23 \mathrm{~mol}^{-1}\right\}$

Curie $(\mathrm{Ci})=3.7 \mathrm{E}+10$ disintegrations /second

Atomic Mass $=$ grams $/ \mathrm{mol}$

The atomic masses are entered into the spreadsheet rounded to 6 decimal places. Avogadro's value is entered to 4 places. The natural $\log$ of 2 is entered to 5 places. Calculated values are rounded to 4 decimal places. Fission product yields are entered and specific heat values are entered as reported in the literature.

\section{Summary}

An updated tabulation of radionuclidic specific activities and heat has been compiled. The use of the table is dependent upon the user. Subsets can be generated by the compiler upon request. 


\section{References}

1. Table of Isotopes, $8^{\text {th }}$ Edition, R. B. Firestone, et al, John Wiley \& Sons, Inc., New York, NY, 1996.

2. The 1995 Update to the Atomic Mass Evaluation, G. Audi and A. H. Wapstra, Nuclear Physics, A595, Vol. 4, 409-480, Dec. 25, 1995.

3. Integrated Data Base Report - 1995: U. S. Spent Nuclear Fuel and Radioactive Waste Inventories, Projections, and Characteristics, Table B.1, DOE/RW-0006, Rev. 12, December 1996.

4. http://www.dne.bni.gov/CoN/index.html, Chart of the Nuclides, Brookhaven National Laboratory and Korean Atomic Energy Agency.

5. http://ie.Ibl.gov/toi.htm , LBNL Isotopes Project - LUNDS Universitet, Nuclear Data Dissemination, Lawrence Berkeley National Laboratory.

6. Database from Nuclide Navigator $\odot$ by EGG/Ortec, now Perkin Elmer, containing data from Topical Presentations in Nuclear Chemistry, Volume 7, The Gamma Rays of the Radionuclides, Verlag Chemie, Weinheim - New York, 1979

7. Nuclides and Isotopes, Fifteenth Edition: Chart of the Nuclides, General Electric Co. and KAPL, Inc., 1996. 


\begin{tabular}{|c|c|c|c|c|c|c|c|c|c|}
\hline Isotope & $\begin{array}{l}\text { Atomic } \\
\text { Mass }\end{array}$ & Halflife & $\begin{array}{l}\text { Time } \\
\text { Units }\end{array}$ & $t_{1 / 2}, \mathrm{sec}$ & Curies/gram & Grams/ Curie & $\begin{array}{c}\text { Watts/Cl } \\
\text { (Ref.3) }\end{array}$ & $\begin{array}{c}\text { Individual }^{1} \\
\text { Fission } \\
\text { Product Yleld } \\
\text { (Ref.4) }\end{array}$ & $\begin{array}{c}\text { Cumulative }^{1} \\
\text { Fission Product } \\
\text { Yleld (Ref.4) }\end{array}$ \\
\hline $\mathrm{H3}$ & 3.0161 & $1.233 E+01$ & year & $3.89 \mathrm{E}+08$ & $9.6130 E+03$ & $1.0403 \mathrm{E}-04$ & 0.000034 & & \\
\hline $\mathrm{He} 6$ & 6.0189 & $8.067 \mathrm{E}-01$ & second & 8.07E-01 & $2.3235 \mathrm{E}+12$ & $4.3038 \mathrm{E}-13$ & & & \\
\hline $\mathrm{He} 8$ & 8.0339 & $1.190 \mathrm{E}-01$ & second & 1.19E-01 & $1.1801 \mathrm{E}+13$ & $8.4742 E-14$ & & & \\
\hline Li 8 & 8.0225 & $8.380 \mathrm{E}-01$ & second & $8.38 \mathrm{E}-01$ & $1.6781 E+12$ & $5.9590 \mathrm{E}-13$ & & & \\
\hline Li 9 & 9.0268 & $1.780 \mathrm{E}-01$ & second & $1.78 \mathrm{E}-01$ & $7.0214 E+12$ & $1.4242 \mathrm{E}-13$ & & & \\
\hline Li 11 & 11.0438 & $8.500 E-03$ & second & $8.50 \mathrm{E}-03$ & $1.2018 \mathrm{E}+14$ & $8.3207 \mathrm{E}-15$ & & & \\
\hline $\mathrm{Be} 7$ & 7.0169 & $5.329 E+01$ & day & $4.60 \mathrm{E}+06$ & $3.4920 E+05$ & $2.8637 \mathrm{E}-06$ & & & \\
\hline Be 10 & 10.0135 & $1.600 \mathrm{E}+06$ & year & $5.05 E+13$ & $2.2313 \mathrm{E}-02$ & $4.4817 \mathrm{E}+01$ & & & \\
\hline $\mathrm{Be} 11$ & 11.0217 & $1.381 \mathrm{E}+01$ & second & $1.38 \mathrm{E}+01$ & $7.4120 \mathrm{E}+10$ & $1.3492 \mathrm{E}-11$ & & & \\
\hline $\mathrm{Be} 12$ & 12.0269 & $2.360 \mathrm{E}-02$ & second & $2.36 \mathrm{E}-02$ & $3.9748 \mathrm{E}+13$ & $2.5159 \mathrm{E}-14$ & & & \\
\hline B 8 & 8.0246 & $7.700 \mathrm{E}-01$ & second & $7.70 E-01$ & $1.8258 \mathrm{E}+12$ & $5.4769 \mathrm{E}-13$ & & & \\
\hline B 12 & 12.0144 & $2.020 \mathrm{E}-02$ & second & $2.02 \mathrm{E}-02$ & $4.6486 \mathrm{E}+13$ & $2.1512 \mathrm{E}-14$ & & & \\
\hline B 13 & 13.0178 & $1.736 \mathrm{E}-02$ & second & $1.74 \mathrm{E}-02$ & $4.9922 \mathrm{E}+13$ & $2.0031 \mathrm{E}-14$ & & & \\
\hline B 14 & 14.0425 & $1.380 \mathrm{E}-02$ & second & $1.38 \mathrm{E}-02$ & $5.8217 E+13$ & $1.7177 \mathrm{E}-14$ & & & \\
\hline C 9 & 9.0310 & $1.265 \mathrm{E}-01$ & second & $1.27 \mathrm{E}-01$ & $9.8753 \mathrm{E}+12$ & $1.0126 \mathrm{E}-13$ & & & \\
\hline$C 10$ & 10.0169 & $1.926 \mathrm{E}+01$ & second & $1.93 \mathrm{E}+01$ & $5.8493 E+10$ & $1.7096 \mathrm{E}-11$ & & & \\
\hline C 11 & 11.0114 & $2.039 E+01$ & minute & $1.22 E+03$ & $8.3746 E+08$ & 1.1941E-09 & & & \\
\hline C 14 & 14.0032 & $5.730 E+03$ & year & $1.81 \mathrm{E}+11$ & $4.4553 E+00$ & $2.2445 \mathrm{E}-01$ & 0.000293 & & \\
\hline C 15 & 15.0106 & $2.449 \mathrm{E}+00$ & second & $2.45 \mathrm{E}+00$ & $3.0689 \mathrm{E}+11$ & $3.2584 \mathrm{E}-12$ & & & \\
\hline C 16 & 16.0147 & $7.470 \mathrm{E}-01$ & second & $7.47 \mathrm{E}-01$ & $9.4306 \mathrm{E}+11$ & $1.0604 \mathrm{E}-12$ & & & \\
\hline N 12 & 12.0186 & $1.100 \mathrm{E}-02$ & second & $1.10 \mathrm{E}-02$ & $8.5335 E+13$ & $1.1718 \mathrm{E}-14$ & & & \\
\hline N13 & 13.0057 & $9.965 E+00$ & minute & $5.98 \mathrm{E}+02$ & $1.4508 E+09$ & $6.8927 E-10$ & & & \\
\hline N 16 & 16.0061 & $7.130 \mathrm{E}+00$ & second & $7.13 E+00$ & $9.8856 \mathrm{E}+10$ & $1.0116 \mathrm{E}-11$ & & & \\
\hline N17 & 17.0085 & $4.173 E+00$ & second & $4.17 E+00$ & $1.5895 E+11$ & $6.2912 E-12$ & & & \\
\hline N18 & 18.0141 & $6.240 \mathrm{E}-01$ & second & $6.24 \mathrm{E}-01$ & $1.0036 E+12$ & $9.9637 \mathrm{E}-13$ & & & \\
\hline 013 & 13.0248 & $8.580 \mathrm{E}-03$ & second & $8.58 \mathrm{E}-03$ & $1.0095 \mathrm{E}+14$ & $9.9056 \mathrm{E}-15$ & & & \\
\hline 014 & 14.0086 & $7.061 \mathrm{E}+01$ & second & $7.06 \mathrm{E}+01$ & $1.1406 \mathrm{E}+10$ & $8.7672 \mathrm{E}-11$ & & & \\
\hline 015 & 15.0031 & $2.037 E+00$ & minute & $1.22 \mathrm{E}+02$ & $6.1525 E+09$ & $1.6253 \mathrm{E}-10$ & & & \\
\hline 019 & 19.0036 & $2.691 \mathrm{E}+01$ & second & $2.69 \mathrm{E}+01$ & $2.2061 \mathrm{E}+10$ & $4.5329 \mathrm{E}-11$ & & & \\
\hline 020 & 20.0040 & $1.352 E+01$ & second & $1.35 \mathrm{E}+01$ & $4.1729 E+10$ & $2.3964 \mathrm{E}-11$ & & & \\
\hline F 17 & 17.0021 & $6.449 E+01$ & second & $6.45 E+01$ & $1.0289 \mathrm{E}+10$ & $9.7189 \mathrm{E}-11$ & & & \\
\hline F 18 & 18.0009 & $1.830 \mathrm{E}+00$ & hour & $6.59 \mathrm{E}+03$ & $9.5158 \mathrm{E}+07$ & $1.0509 \mathrm{E}-08$ & & & \\
\hline$F 20$ & 20.0000 & $1.100 \mathrm{E}+01$ & second & $1.10 E+01$ & $5.1281 \mathrm{E}+10$ & $1.9501 \mathrm{E}-11$ & & & \\
\hline$F 21$ & 20.9999 & $4.158 \mathrm{E}+00$ & second & $4.16 \mathrm{E}+00$ & $1.2920 \mathrm{E}+11$ & $7.7397 E-12$ & & & \\
\hline F22 & 22.0030 & $4.230 \mathrm{E}+00$ & second & $4.23 \mathrm{E}+00$ & $1.2121 \mathrm{E}+11$ & $8.2498 \mathrm{E}-12$ & & & \\
\hline
\end{tabular}

WSRC-TR-99-00344

October, 1999

page 4 


\begin{tabular}{|c|c|c|c|c|c|c|c|c|c|}
\hline Isotope & $\begin{array}{l}\text { Atomic } \\
\text { Mass }\end{array}$ & Halflife & $\begin{array}{l}\text { Time } \\
\text { Units }\end{array}$ & $t_{1 / 2}, \sec$ & Curies/gram & Grams/ Curie & $\begin{array}{c}\text { Watts/Ci } \\
\text { (Ref.3) }\end{array}$ & $\begin{array}{c}\text { Individual }^{1} \\
\text { Fission } \\
\text { Product Yield } \\
\text { (Ref.4) }\end{array}$ & $\begin{array}{c}\text { Cumulative }^{1} \\
\text { Fission Product } \\
\text { Yield (Ref.4) }\end{array}$ \\
\hline F 23 & 23.0036 & $2.230 E+00$ & second & $2.23 E+00$ & $2.1993 E+11$ & $4.5470 \mathrm{E}-12$ & & & \\
\hline $\mathrm{Ne} 17$ & 17.0177 & $1.092 \mathrm{E}-01$ & second & $1.09 \mathrm{E}-01$ & $6.0709 E+12$ & $1.6472 \mathrm{E}-13$ & & & \\
\hline$\overline{\mathrm{Ne} 18}$ & 18.0057 & $1.672 E+00$ & second & $1.67 \mathrm{E}+00$ & $3.7474 E+11$ & $2.6685 \mathrm{E}-12$ & & & \\
\hline$\overline{\mathrm{Ne} 19}$ & 19.0014 & $1.735 E+01$ & second & $1.73 E+01$ & $3.4223 E+10$ & $2.9220 \mathrm{E}-11$ & & & \\
\hline $\mathrm{Ne} 23$ & 22.9945 & $3.724 \mathrm{E}+01$ & second & $3.72 \mathrm{E}+01$ & $1.3175 E+10$ & $7.5903 E-11$ & & & \\
\hline $\mathrm{Ne} 24$ & 23.9936 & $3.380 E+00$ & minute & $2.03 E+02$ & $2.3185 E+09$ & $4.3131 \mathrm{E}-10$ & & & \\
\hline $\mathrm{Ne} 25$ & 24.9978 & $6.020 \mathrm{E}-01$ & second & $6.02 \mathrm{E}-01$ & $7.4968 \mathrm{E}+11$ & $1.3339 \mathrm{E}-12$ & & & \\
\hline $\mathrm{Na} 20$ & 20.0073 & $4.479 \mathrm{E}-01$ & second & $4.48 E-01$ & $1.2589 \mathrm{E}+12$ & $7.9432 E-13$ & & & \\
\hline $\mathrm{Na} 21$ & 20.9977 & $2.249 E+01$ & second & $2.25 E+01$ & $2.3890 \mathrm{E}+10$ & $4.1859 \mathrm{E}-11$ & & & \\
\hline$\overline{\mathrm{Na} 22}$ & 21.9944 & $2.602 E+00$ & year & $8.21 E+07$ & $6.2468 \mathrm{E}+03$ & $1.6008 E-04$ & & & \\
\hline $\mathrm{Na} 24$ & 23.9910 & $1.496 E+01$ & hour & $5.39 E+04$ & $8.7322 \mathrm{E}+06$ & $1.1452 \mathrm{E}-07$ & & & \\
\hline $\mathrm{Na} 24 \mathrm{~m}$ & 23.9910 & $2.020 \mathrm{E}-02$ & second & $2.02 E-02$ & $2.3280 E+13$ & $4.2956 \mathrm{E}-14$ & & & \\
\hline $\mathrm{Na} 25$ & 24.9900 & $5.910 E+01$ & second & $5.91 E+01$ & $7.6388 E+09$ & $1.3091 \mathrm{E}-10$ & & & \\
\hline $\mathrm{Na} 26$ & 25.9926 & $1.072 E+00$ & second & $1.07 E+00$ & $4.0489 E+11$ & $2.4698 \mathrm{E}-12$ & & & \\
\hline $\mathrm{Na} 27$ & 26.9940 & $3.010 \mathrm{E}-01$ & second & $3.01 \mathrm{E}-01$ & $1.3885 E+12$ & $7.2021 \mathrm{E}-13$ & & & \\
\hline $\mathrm{Na} 28$ & 27.9989 & $3.050 E-02$ & second & $3.05 E-02$ & $1.3211 \mathrm{E}+13$ & $7.5694 E-14$ & & & \\
\hline $\mathrm{Na29}$ & 29.0028 & $4.490 \mathrm{E}-02$ & second & $4.49 \mathrm{E}-02$ & $8.6634 \mathrm{E}+12$ & $1.1543 \mathrm{E}-13$ & & & \\
\hline $\mathrm{Na} 30$ & 30.0092 & $4.800 E-02$ & second & $4.80 \mathrm{E}-02$ & $7.8321 E+12$ & $1.2768 \mathrm{E}-13$ & & & \\
\hline$\overline{\mathrm{Na} 31}$ & 31.0136 & $1.700 \mathrm{E}-02$ & second & $1.70 \mathrm{E}-02$ & $2.1398 E+13$ & $4.6733 \mathrm{E}-14$ & & & \\
\hline $\mathrm{Na} 32$ & 32.0196 & $1.320 \mathrm{E}-02$ & second & $1.32 E-02$ & $2.6692 E+13$ & $3.7464 \mathrm{E}-14$ & & & \\
\hline $\mathrm{Na} 33$ & 33.0274 & $8.200 \mathrm{E}-03$ & second & $8.20 E-03$ & $4.1657 \mathrm{E}+13$ & $2.4006 E-14$ & & & \\
\hline $\mathrm{Mg} 20$ & 20.0189 & $9.500 \mathrm{E}-02$ & second & $9.50 \mathrm{E}-02$ & $5.9322 \mathrm{E}+12$ & $1.6857 \mathrm{E}-13$ & & & \\
\hline $\mathrm{Mg} 21$ & 21.0117 & $1.220 \mathrm{E}-01$ & second & $1.22 E-01$ & $4.4010 E+12$ & $2.2722 \mathrm{E}-13$ & & & \\
\hline $\mathrm{Mg} 22$ & 21.9996 & $3.857 E+00$ & second & $3.86 E+00$ & $1.3296 \mathrm{E}+11$ & $7.5212 \mathrm{E}-12$ & & & \\
\hline $\mathrm{Mg} 23$ & 22.9941 & $1.117 E+01$ & second & $1.12 E+01$ & $4.3924 E+10$ & $2.2766 \mathrm{E}-11$ & & & \\
\hline$\overline{\mathrm{Mg} 27}$ & 26.9843 & $9.462 E+00$ & minute & $5.68 E+02$ & $7.3643 E+08$ & $1.3579 \mathrm{E}-09$ & & & \\
\hline $\mathrm{Mg} 28$ & 27.9839 & $2.130 E+01$ & hour & $7.67 E+04$ & $5.2576 \mathrm{E}+06$ & $1.9020 \mathrm{E}-07$ & & & \\
\hline$M g 29$ & 28.9886 & $1.300 \mathrm{E}-03$ & second & $1.30 \mathrm{E}-03$ & $2.9937 E+14$ & $3.3404 \mathrm{E}-15$ & & & \\
\hline $\mathrm{Mg} 30$ & 29.9905 & $3.300 \mathrm{E}-01$ & second & $3.30 \mathrm{E}-01$ & $1.1399 \mathrm{E}+12$ & $8.7724 \mathrm{E}-13$ & & & \\
\hline $\mathrm{Mg} 31$ & 30.9965 & $2.500 \mathrm{E}-01$ & second & $2.50 E-01$ & $1.4559 \mathrm{E}+12$ & $6.8687 \mathrm{E}-13$ & & & \\
\hline Al 24 & 23.9999 & $2.053 E+00$ & second & $2.05 E+00$ & $2.2897 \mathrm{E}+11$ & $4.3674 \mathrm{E}-12$ & & & \\
\hline$A \mid 24 m$ & 23.9999 & $1.313 \mathrm{E}-01$ & second & $1.31 \mathrm{E}-01$ & $3.5802 E+12$ & $2.7932 \mathrm{E}-13$ & & & \\
\hline$\overline{A l 25}$ & 24.9904 & $7.183 E+00$ & second & $7.18 \mathrm{E}+00$ & $6.2849 \mathrm{E}+10$ & $1.5911 \mathrm{E}-11$ & & & \\
\hline Al 26 & 25.9869 & $7: 400 E+05$ & year & $2.34 E+13$ & $1.8590 \mathrm{E}-02$ & $5.3793 E+01$ & 0.018250 & & \\
\hline Al $26 \mathrm{~m}$ & 25.9869 & $6.345 \mathrm{E}+00$ & second & $6.35 \mathrm{E}+00$ & $6.8419 \mathrm{E}+10$ & $1.4616 \mathrm{E}-11$ & & & \\
\hline
\end{tabular}

WSRC-TR-99-00344

October, 1999

page 5 
Radionuclide Specific Data

\begin{tabular}{|c|c|c|c|c|c|c|c|c|c|}
\hline Isotope & $\begin{array}{l}\text { Atomic } \\
\text { Mass }\end{array}$ & Halflife & $\begin{array}{l}\text { Time } \\
\text { Units }\end{array}$ & $t_{12}, \sec$ & Curies/gram & Grams/ Curle & $\begin{array}{c}\text { Watts/Ci } \\
\text { (Ref.3) }\end{array}$ & $\begin{array}{c}\text { Individual }^{1} \\
\text { Flssion } \\
\text { Product Yield } \\
(\text { Ref.4) } \\
\end{array}$ & $\begin{array}{l}\text { Cumulative }^{9} \\
\text { Fission Product } \\
\text { Yield (Ref.4) }\end{array}$ \\
\hline Al 28 & 27.9819 & $2.241 E+00$ & minute & $1.34 \mathrm{E}+02$ & $2.9980 \mathrm{E}+09$ & $3.3356 \mathrm{E}-10$ & & & \\
\hline Al 29 & 28.9804 & $6.560 E+00$ & minute & $3.94 E+02$ & $9.8905 \mathrm{E}+08$ & $1.0111 \mathrm{E}-09$ & & & \\
\hline A) 30 & 29.9830 & $3.600 E+00$ & second & $3.60 E+00$ & $1.0452 E+11$ & $9.5675 \mathrm{E}-12$ & & & \\
\hline Al 31 & 30.9839 & $6.440 \mathrm{E}-01$ & second & $6.44 \mathrm{E}-01$ & $5.6540 \mathrm{E}+11$ & $1.7687 \mathrm{E}-12$ & & & \\
\hline Si 25 & 25.0041 & $2.200 \mathrm{E}-01$ & second & $2.20 \mathrm{E}-01$ & $2.0509 \mathrm{E}+12$ & $4.8759 \mathrm{E}-13$ & & & \\
\hline Si 26 & 25.9923 & $2.234 \mathrm{E}+00$ & second & $2.23 E+00$ & $1.9429 \mathrm{E}+11$ & $5.1470 \mathrm{E}-12$ & & & \\
\hline Si 27 & 26.9867 & $4.160 E+00$ & second & $4.16 \mathrm{E}+00$ & $1.0049 \mathrm{E}+11$ & $9.9510 \mathrm{E}-12$ & & & \\
\hline Si 31 & 30.9754 & $2.622 \mathrm{E}+00$ & hour & $9.44 E+03$ & $3.8586 \mathrm{E}+07$ & $2.5916 \mathrm{E}-08$ & & & \\
\hline Si 32 & 31.9741 & $1.500 E+02$ & year & $4.73 \mathrm{E}+09$ & $7.4537 \mathrm{E}+01$ & $1.3416 \mathrm{E}-02$ & 0.001245 & & \\
\hline Si 33 & 32.9780 & $6.180 E+00$ & second & $6.18 \mathrm{E}+00$ & $5.5356 E+10$ & $1.8065 \mathrm{E}-11$ & & & \\
\hline$P 28$ & 27.9923 & $2.703 E-01$ & second & $2.70 \mathrm{E}-01$ & $1.4910 \mathrm{E}+12$ & $6.7067 \mathrm{E}-13$ & & & \\
\hline $\mathrm{P} 29$ & 28.9818 & $4.140 \mathrm{E}+00$ & second & $4.14 E+00$ & $9.4027 \mathrm{E}+10$ & $1.0635 \mathrm{E}-11$ & & & \\
\hline P30 & 29.9783 & $2.498 \mathrm{E}+00$ & minute & $1.50 \mathrm{E}+02$ & $2.5109 \mathrm{E}+09$ & $3.9827 \mathrm{E}-10$ & & & \\
\hline P 32 & 31.9739 & $1.426 \mathrm{E}+01$ & day & $1.23 \mathrm{E}+06$ & $2.8634 \mathrm{E}+05$ & $3.4923 \mathrm{E}-06$ & 0.005120 & & \\
\hline$P 33$ & 32.9717 & $2.534 \mathrm{E}+01$ & day & $2.19 \mathrm{E}+06$ & $1.5628 \mathrm{E}+05$ & $6.3986 \mathrm{E}-06$ & & & \\
\hline P34 & 33.9736 & $1.243 E+01$ & second & $1.24 E+01$ & $2.6716 \mathrm{E}+10$ & $3.7431 \mathrm{E}-11$ & & & \\
\hline P35 & 34.9733 & $4.730 E+01$ & second & $4.73 E+01$ & $6.8199 \mathrm{E}+09$ & $1.4663 \mathrm{E}-10$ & & & \\
\hline 529 & 28.9966 & $1.870 E-01$ & second & $1.87 \mathrm{E}-01$ & $2.0806 \mathrm{E}+12$ & $4.8063 \mathrm{E}-13$ & & & \\
\hline 530 & 29.9849 & $1.178 \mathrm{E}+00$ & second & $1.18 \mathrm{E}+00$ & $3.1940 \mathrm{E}+11$ & $3.1309 \mathrm{E}-12$ & & & \\
\hline 531 & 30.9796 & $2.572 \mathrm{E}+00$ & second & $2.57 E+00$ & $1.4159 \mathrm{E}+11$ & $7.0627 \mathrm{E}-12$ & & & \\
\hline S 35 & 34.9690 & $8.751 E+01$ & day & $7.56 \mathrm{E}+06$ & $4.2670 \mathrm{E}+04$ & $2.3436 \mathrm{E}-05$ & 0.000288 & & \\
\hline 537 & 36.9711 & $5.050 \mathrm{E}+00$ & minute & $3.03 E+02$ & $1.0071 \mathrm{E}+09$ & $9.9295 \mathrm{E}-10$ & & & \\
\hline 538 & 37.9712 & $2.838 E+00$ & hour. & $1.02 E+04$ & $2.9081 E+07$ & $3.4387 \mathrm{E}-08$ & & & \\
\hline $\mathrm{Cl} 32$ & 31.9857 & $2.980 E-01$ & second & $2.98 \mathrm{E}-01$ & $1.1836 \mathrm{E}+12$ & $8.4488 \mathrm{E}-13$ & & & \\
\hline Cl 33 & 32.9775 & $2.511 \mathrm{E}+00$ & second & $2.51 E+00$ & $1.3624 E+11$ & $7.3399 \mathrm{E}-12$ & & & \\
\hline $\mathrm{Cl} 34$ & 33.9738 & $1.526 \mathrm{E}+00$ & second & $1.53 E+00$ & $2.1755 \mathrm{E}+11$ & $4.5966 \mathrm{E}-1.2$ & & & \\
\hline $\mathrm{Cl} 34 \mathrm{~m}$ & 33.9738 & $3.200 E+0.1$ & minute & $1.92 \mathrm{E}+03$ & $1.7295 E+08$ & $5.7819 \mathrm{E}-09$ & & & \\
\hline Cl 36 & 35.9683 & $3.010 E+05$ & year & $9.50 \mathrm{E}+12$ & $3.3020 \mathrm{E}-02$ & $3.0285 \mathrm{E}+01$ & 0.001458 & & \\
\hline Cl 38 & 37.9680 & $3.724 E+01$ & minute & $2.23 E+03$ & $1.3298 \mathrm{E}+08$ & $7.5197 \mathrm{E}-09$ & & & \\
\hline $\mathrm{Cl} 38 \mathrm{~m}$ & 37.9680 & $7.150 \mathrm{E}-01$ & second & $7.15 \mathrm{E}-01$ & $4.1558 \mathrm{E}+11$ & $2.4063 \mathrm{E}-12$ & & & \\
\hline Cl 39 & 38.9680 & $5.560 \mathrm{E}+01$ & minute & $3.34 \mathrm{E}+03$ & $8.6785 E+07$ & $1.1523 E-08$ & & & \\
\hline $\mathrm{Cl} 40$ & 39.9704 & $1.350 E+00$ & minute & $8.10 \mathrm{E}+01$ & $3.4846 \mathrm{E}+09$ & $2.8698 \mathrm{E}-10$ & & & \\
\hline Cl 41 & 40.9707 & $3.840 E+01$ & second & $3.84 E+01$ & $7.1709 E+09$ & 1.3945E-10 & & & \\
\hline $\operatorname{Ar} 33$ & 32.9899 & $1.730 \mathrm{E}-01$ & second & $1.73 \mathrm{E}-01$ & $1.9767 \mathrm{E}+12$ & $5.0588 \mathrm{E}-13$ & & & \\
\hline $\operatorname{Ar} 34$ & 33.9803 & $8.440 \mathrm{E}-01$ & second & $8.44 E-01$ & $3.9338 \mathrm{E}+11$ & $2.5421 \mathrm{E}-12$ & & & \\
\hline
\end{tabular}

WSRC-TR-99-00344

October, 1999

page 6 


\begin{tabular}{|c|c|c|c|c|c|c|c|c|c|}
\hline Isotope & $\begin{array}{c}\text { Atomic } \\
\text { Mass }\end{array}$ & Halflife & $\begin{array}{l}\text { Time } \\
\text { Units }\end{array}$ & $t_{1 / 2}, \mathrm{sec}$ & Curies/ gram & Grams/ Curie & $\begin{array}{c}\text { Watts/Ci } \\
\text { (Ref.3) }\end{array}$ & \begin{tabular}{|c|} 
Individual $^{4}$ \\
Fission \\
Product Yield \\
(Ref.4) \\
\end{tabular} & $\begin{array}{c}\text { Cumulative }^{4} \\
\text { Fission Product } \\
\text { Yield (Ref.4) }\end{array}$ \\
\hline $\operatorname{Ar} 35$ & 34.9753 & $1.775 E+00$ & second & $1.78 E+00$ & $1.8173 E+11$ & $5.5028 \mathrm{E}-12$ & & & \\
\hline $\operatorname{Ar} 37$ & 36.9668 & $3 . \overline{504 E+01}$ & day & $3.03 E+06$ & $1.0081 E+05$ & $9.9200 \mathrm{E}-06$ & & & \\
\hline $\operatorname{Ar} 39$ & 38.9643 & $2.690 \mathrm{E}+02$ & year & $8.49 E+09$ & $3.4107 \mathrm{E}+01$ & $2.9320 \mathrm{E}-02$ & & & \\
\hline $\operatorname{Ar} 41$ & 40.9645 & $1.822 \mathrm{E}+00$ & hour & $6.56 \mathrm{E}+03$ & $4.1980 \mathrm{E}+07$ & $2.3821 \mathrm{E}-08$ & & & \\
\hline$\overline{\operatorname{Ar} 42}$ & 41.9630 & $3.290 E+01$ & year & $1.04 E+09$ & $2.5894 E+02$ & $3.8619 E-03$ & & & \\
\hline $\operatorname{Ar} 43$ & 42.9657 & $5.370 \mathrm{E}+00$ & minute & $3.22 E+02$ & $8.1495 \mathrm{E}+08$ & $1.2271 \mathrm{E}-09$ & & & \\
\hline Ar 44 & 43.9654 & $1.187 E+01$ & minute & $7.12 \mathrm{E}+02$ & $3.6030 \mathrm{E}+08$ & $2.7755 \mathrm{E}-09$ & & & \\
\hline $\operatorname{Ar} 45$ & 44.9607 & $2.148 E+01$ & second & $2.15 E+01$ & $1.1682 E+10$ & $8.5603 \mathrm{E}-11$ & & & \\
\hline $\operatorname{Ar} 46$ & 45.9681 & $8.400 E+00$ & second & $8.40 \mathrm{E}+00$ & $2.9217 \mathrm{E}+10$ & $3.4226 \mathrm{E}-11$ & & & \\
\hline $\mathrm{K} 35$ & 34.9880 & $1.900 \mathrm{E}-01$ & second & $1.90 \mathrm{E}-01$ & $1.6971 \mathrm{E}+12$ & $5.8925 \mathrm{E}-13$ & & & \\
\hline K 36 & 35.9813 & $3.420 \mathrm{E}-01$ & second & $3.42 \mathrm{E}-01$ & $9.1680 E+11$ & $1.0908 \mathrm{E}-12$ & & & \\
\hline K 37 & 36.9734 & $1.226 \mathrm{E}+00$ & second & $1.23 E+00$ & $2.4888 E+11$ & $4.0179 \mathrm{E}-12$ & & & \\
\hline K38 & 37.9691 & $7.636 \mathrm{E}+00$ & minute & $4.58 E+02$ & $6.4853 E+08$ & $1.5420 \mathrm{E}-09$ & & & \\
\hline $\mathrm{K} 38 \mathrm{~m}$ & 37.9691 & $9.239 \mathrm{E}-01$ & second & $9.24 \mathrm{E}-01$ & $3.2160 \mathrm{E}+11$ & $3.1094 \mathrm{E}-12$ & & & \\
\hline $\mathrm{K} 40$ & 39.9640 & $1.277 E+09$ & year & $4.03 E+16$ & $7.0049 \mathrm{E}-06$ & $1.4276 E+05$ & 0.003620 & & \\
\hline $\mathrm{K} 42$ & 41.9624 & $1.236 \mathrm{E}+01$ & hour & $4.45 E+04$ & $6.0422 \mathrm{E}+06$ & $1.6550 \mathrm{E}-07$ & & & \\
\hline $\mathrm{K} 43$ & 42.9607 & $2.230 E+01$ & hour & $8.03 E+04$ & $3.2711 \mathrm{E}+06$ & $3.0570 \mathrm{E}-07$ & & & \\
\hline $\mathrm{K} 44$ & 43.9616 & $2.213 E+01$ & minute & $1.33 E+03$ & $1.9327 \mathrm{E}+08$ & $5.1740 \mathrm{E}-09$ & & & \\
\hline K 45 & 44.9607 & $1.730 E+01$ & minute & $1.04 \mathrm{E}+03$ & $2.4174 \mathrm{E}+08$ & $4.1367 \mathrm{E}-09$ & & & \\
\hline $\mathrm{K} 46$ & 45.9620 & $1.750 \mathrm{E}+00$ & minute & $1.05 \mathrm{E}+02$ & $2.3377 E+09$ & $4.2777 \mathrm{E}-10$ & & & \\
\hline $\mathrm{K} 47$ & 46.9617 & $1.750 E+01$ & second & $1.75 \mathrm{E}+01$ & $1.3728 \mathrm{E}+10$ & $7.2846 \mathrm{E}-11$ & & & \\
\hline K 48 & 47.9655 & $6.800 \mathrm{E}+00$ & second & $6.80 E+00$ & $3.4589 \mathrm{E}+10$ & $2.8911 \mathrm{E}-11$ & & & \\
\hline K49 & 48.9675 & $1.260 E+00$ & second & $1.26 E+00$ & $1.8285 E+11$ & $5.4689 \mathrm{E}-12$ & & & \\
\hline K50 & 49.9728 & $4.720 \mathrm{E}-01$ & second & $4.72 \mathrm{E}-01$ & $4.7830 \mathrm{E}+11$ & $2.0907 \mathrm{E}-12$ & & & \\
\hline K51 & 50.9764 & $3.650 \mathrm{E}-01$ & second & $3.65 \mathrm{E}-01$ & $6.0634 \mathrm{E}+11$ & $1.6492 \mathrm{E}-12$ & & & \\
\hline K 52 & 51.9826 & $1.050 \mathrm{E}-01$ & second & $1.05 E-01$ & $2.0669 E+12$ & $4.8381 \mathrm{E}-13$ & & & \\
\hline $\mathrm{K} 53$ & 52.9871 & $3.000 \mathrm{E}-02$ & second & $3.00 \mathrm{E}-02$ & $7.0972 \mathrm{E}+12$ & $1.4090 \mathrm{E}-13$ & & & \\
\hline K54 & 53.9940 & $1.000 \mathrm{E}-02$ & second & $1.00 \mathrm{E}-02$ & $2.0894 \mathrm{E}+13$ & $4.7860 \mathrm{E}-14$ & & & \\
\hline $\mathrm{Ca} 37$ & 36.9859 & $1.750 \mathrm{E}-01$ & second & $1.75 \mathrm{E}-01$ & $1.7430 \mathrm{E}+12$ & $5.7372 \mathrm{E}-13$ & & & \\
\hline $\mathrm{Ca} 38$ & 37.9763 & $4.400 \mathrm{E}-01$ & second & $4.40 \mathrm{E}-01$ & $6.7517 \mathrm{E}+11$ & $1.4811 E-12$ & & & \\
\hline $\mathrm{Ca} 39$ & 38.9707 & $8.596 \mathrm{E}-01$ & second & $8.60 \mathrm{E}-01$ & $3.3678 \mathrm{E}+11$ & $2.9693 \mathrm{E}-12$ & & & \\
\hline $\mathrm{Ca} 41$ & 40.9623 & $1.030 E+05$ & year & $3.25 \mathrm{E}+12$ & $8.4730 \mathrm{E}-02$ & $1.1802 \mathrm{E}+01$ & & & \\
\hline $\mathrm{Ca} 45$ & 44.9562 & $1.638 \mathrm{E}+02$ & day & $1.42 E+07$ & $1.7732 E+04$ & $5.6395 \mathrm{E}-05$ & 0.000456 & & \\
\hline $\mathrm{Ca} 47$ & 46.9545 & $4.536 \mathrm{E}+00$ & day & $3.92 \mathrm{E}+05$ & $6.1307 \mathrm{E}+05$ & $1.6311 \mathrm{E}-06$ & & & \\
\hline Ca 49 & 48.9557 & $8.715 E+00$ & minute & $5.23 E+02$ & $4.4071 \mathrm{E}+08$ & $2.2691 \mathrm{E}-09$ & & & \\
\hline
\end{tabular}

WSRC-TR-99-00344

October, 1999

page 7 
Radionuclide Specific Data

\begin{tabular}{|c|c|c|c|c|c|c|c|c|c|}
\hline Isotope & $\begin{array}{c}\text { Atomic } \\
\text { Mass }\end{array}$ & Halflife & $\begin{array}{l}\text { Time } \\
\text { Units }\end{array}$ & $t_{1 / 2}$, sec & Curies/gram & Grams/ Curie & $\begin{array}{c}\text { Watts/Cl } \\
\text { (Ref.3) }\end{array}$ & $\begin{array}{c}\text { Individual }^{1} \\
\text { Fission } \\
\text { Product Yield } \\
\text { (Ref.4) }\end{array}$ & $\begin{array}{l}\text { Cumulative } \\
\text { Fission Product } \\
\text { Yield (Ref.4) }\end{array}$ \\
\hline Ca 50 & 49.9575 & $1.390 E+01$ & second & $1.39 \mathrm{E}+01$ & $1.6247 \mathrm{E}+10$ & $6.1552 E-11$ & & & \\
\hline Sc 40 & 39.9780 & $1.823 E-01$ & second & $1.82 E-01$ & $1.5480 \mathrm{E}+12$ & $6.4600 \mathrm{E}-13$ & & & \\
\hline Sc 41 & 40.9693 & $5.963 E-01$ & second & $5.96 E-01$ & $4.6180 \mathrm{E}+11$ & $2.1654 E-12$ & & & \\
\hline $\mathrm{Sc} 42$ & 41.9655 & $6.813 \mathrm{E}-01$ & second & $6.81 \mathrm{E}-01$ & $3.9459 \mathrm{E}+11$ & $2.5343 E-12$ & & & \\
\hline Sc 43 & 42.9612 & $3.891 E+00$ & hour & $1.40 E+04$ & $1.8747 \mathrm{E}+07$ & $5.3341 E-08$ & & & \\
\hline Sc 44 & 43.9594 & $3.927 E+00$ & hour & $1.41 E+04$ & $1.8154 \mathrm{E}+07$ & $5.5086 \mathrm{E}-08$ & & & \\
\hline Sc $44 \mathrm{~m}$ & 43.9594 & $2.440 E+00$ & day & $2.11 E+05$ & $1.2174 \mathrm{E}+06$ & $8.2145 E-07$ & & & \\
\hline Sc 46 & 45.9952 & $8.379 E+01$ & day & $7.24 E+06$ & $3.3881 \mathrm{E}+04$ & $2.9515 \mathrm{E}-05$ & 0.012570 & & \\
\hline Sc $46 \mathrm{~m}$ & 45.9952 & $1.875 E+01$ & second & $1.88 E+01$ & $1.3082 E+10$ & $7.6443 E-11$ & & & \\
\hline Sc 47 & 46.9524 & $3.345 E+00$ & day & $2.89 E+05$ & $8.3140 \mathrm{E}+05$ & $1.2028 \mathrm{E}-06$ & & & \\
\hline So 48 & 47.9522 & $1.820 E+00$ & day & $1.57 \mathrm{E}+05$ & $1.4965 E+06$ & $6.6822 \mathrm{E}-07$ & & & \\
\hline Sc 49 & 48.9500 & $5.720 E+01$ & minute & $3.43 E+03$ & $6.7155 E+07$ & $1.4891 \mathrm{E}-08$ & & & \\
\hline Sc 50 & 49.9522 & $1.708 E+00$ & minute & $1.02 E+02$ & $2.2035 E+09$ & $4.5383 \mathrm{E}-10$ & & & \\
\hline Sc $50 \mathrm{~m}$ & 49.9522 & $3.500 E-01$ & second & $3.50 E-01$ & $6.4529 \mathrm{E}+11$ & $1.5497 \mathrm{E}-12$ & & & \\
\hline Sc 51 & 50.9536 & $1.240 \mathrm{E}+01$ & second & $1.24 \mathrm{E}+01$ & $1.7856 \mathrm{E}+10$ & $5.6004 \mathrm{E}-11$ & & & \\
\hline $\mathrm{Ti} 41$ & 40.9831 & 8.000E-02 & second & $8.00 E-02$ & $3.4410 \mathrm{E}+12$ & $2.9062 E-13$ & & & \\
\hline $\mathrm{Ti} 42$ & 41.9730 & 1.990 E-01 & second & 1.99E-01 & $1.3507 \mathrm{E}+12$ & $7.4037 \mathrm{E}-13$ & & & \\
\hline $\mathrm{Ti} 43$ & 42.9685 & 5.090E-01 & second & 5.09E-01 & $5.1583 E+11$ & $1.9386 \mathrm{E}-12$ & & & \\
\hline $\mathrm{Ti} 44$ & 43.9597 & $4.900 \mathrm{E}+01$ & year & $1.55 \mathrm{E}+09$ & $1.6596 \mathrm{E}+02$ & $6.0255 \mathrm{E}-03$ & & & \\
\hline $\mathrm{Ti} 45$ & 44.9581 & $3.080 E+00$ & hour & $1.11 E+04$ & $2.2632 E+07$ & $4.4186 \mathrm{E}-08$ & & & \\
\hline $\mathrm{Ti} 51$ & 50.9466 & $5.760 E+00$ & minute & $3.46 E+02$ & $6.4075 E+08$ & $1.5607 \mathrm{E}-09$ & & & \\
\hline Ti 52 & 51.9469 & $1.700 E+00$ & minute & $1.02 E+02$ & $2.1292 E+09$ & $4.6966 \mathrm{E}-10$ & & & \\
\hline $\mathrm{Ti} 53$ & 52.9497 & $3.270 \mathrm{E}+01$ & second & $3.27 \mathrm{E}+01$ & $6.5158 \mathrm{E}+09$ & $1.5347 \mathrm{E}-10$ & & & \\
\hline V44 & 43.9744 & $9.000 \mathrm{E}-02$ & second & $9.00 E-02$ & $2.8506 E+12$ & $3.5081 \mathrm{E}-13$ & & & \\
\hline$V 46$ & 45.9602 & 4.224E-01 & second & $4.22 E-01$ & $5.8117 \mathrm{E}+11$ & $1.7207 \mathrm{E}-12$ & & & \\
\hline V47 & 46.9549 & $3.260 \mathrm{E}+01$ & minute & $1.96 \mathrm{E}+03$ & $1.2284 \mathrm{E}+08$ & 8.1409E-09 & & & \\
\hline V 48 & 47.9523 & 1.597E+01 & day & $1.38 E+06$ & $1.7047 \mathrm{E}+05$ & $5.8661 \mathrm{E}-06$ & & & \\
\hline V 49 & 48.9485 & $3.300 E+02$ & day & $2.85 \mathrm{E}+07$ & $8.0837 E+03$ & 1.2371E-04 & & & \\
\hline \begin{tabular}{|l}
$\mathrm{V} 52$ \\
\end{tabular} & 51.9448 & $3.750 \mathrm{E}+00$ & minute & $2.25 E+02$ & $9.6528 \mathrm{E}+08$ & 1.0360 E-09 & & & \\
\hline V53 & 52.9443 & $1.610 \mathrm{E}+00$ & minute & $9.66 E+01$ & $2.2059 E+09$ & $4.5334 \mathrm{E}-10$ & & & \\
\hline V 54 & 53.9464 & $4.980 E+01$ & second & $4.98 E+01$ & $4.1994 \mathrm{E}+09$ & $2.3813 E-10$ & & & \\
\hline $\mathrm{Cr} 45$ & 44.9792 & 5.000E-02 & second & $5.00 \mathrm{E}-02$ & $5.0164 E+12$ & $1.9934 \mathrm{E}-13$ & & & \\
\hline $\mathrm{Cr} 46$ & 45.9684 & $2.600 \mathrm{E}-01$ & second & 2.60E-01 & $9.4394 \mathrm{E}+11$ & $1.0594 E-12$ & & & \\
\hline $\operatorname{Cr} 47$ & 46.9629 & 5.000E-01 & second & $5.00 \mathrm{E}-01$ & $4.8045 \mathrm{E}+11$ & $2.0814 \mathrm{E}-12$ & & & \\
\hline $\mathrm{Cr} 48$ & 47.9540 & $2.300 \mathrm{E}+01$ & hour & $8.28 E+04$ & $2.8413 E+06$ & 3.5195E-07 & & & \\
\hline
\end{tabular}

WSRC-TR-99-00344

October, 1999

page 8 


\begin{tabular}{|c|c|c|c|c|c|c|c|c|c|}
\hline Isotope & $\begin{array}{l}\text { Atomic } \\
\text { Mass }\end{array}$ & Halflife & $\begin{array}{l}\text { Time } \\
\text { Units }\end{array}$ & $t_{1 / 2}, \sec$ & Curies/gram & Grams/ Curie & $\begin{array}{c}\text { Watts/Cl } \\
\text { (Ref.3) }\end{array}$ & $\begin{array}{c}\text { Individual }^{1} \\
\text { Fission }^{-} \\
\text {Product Yield } \\
\text { Ref.4) }^{-}\end{array}$ & $\begin{array}{l}\text { Cumulative } \\
\text { Fission Product } \\
\text { Yield (Ref.4) }\end{array}$ \\
\hline Cr 49 & 48.9513 & $4.230 \mathrm{E}+01$ & minute & $2.54 E+03$ & $9.0807 \mathrm{E}+07$ & $1.1012 \mathrm{E}-08$ & & & \\
\hline $\mathrm{Cr} 51$ & 50.9448 & $2.770 E+01$ & day & $2.39 E+06$ & $9.2523 E+04$ & $1.0808 \mathrm{E}-05$ & 0.000211 & & \\
\hline Cr55 & 54.9408 & $3.497 E+00$ & minute & $2.10 \mathrm{E}+02$ & $9.7867 \mathrm{E}+08$ & $1.0218 \mathrm{E}-09$ & & & \\
\hline Cr 56 & 55.9406 & $5.940 \mathrm{E}+00$ & minute & $3.56 \mathrm{E}+02$ & $5.6586 \mathrm{E}+08$ & 1.7672E-09 & & & \\
\hline Cr 57 & 56.9438 & $2.110 E+01$ & second & $2: 11 E+01$ & $9.3896 E+09$ & $1.0650 \mathrm{E}-10$ & & & \\
\hline $\mathrm{Mn} 49$ & 48.9596 & $3.820 \mathrm{E}-01$ & second & $3.82 E-01$ & $6.0322 E+11$ & $1.6578 \mathrm{E}-12$ & & & \\
\hline $\operatorname{Mn} 50$ & 49.9542 & $2.830 E-01$ & second & $2.83 \mathrm{E}-01$ & $7.9803 E+11$ & $1.2531 \mathrm{E}-12$ & & & \\
\hline Mn 50m & 49.9542 & $1.750 E+00$ & minute & $1.05 \mathrm{E}+02$ & $2.1509 \mathrm{E}+09$ & $4.6493 \mathrm{E}-10$ & & & \\
\hline $\mathrm{Mn} 51$ & 50.9482 & $4.620 E+01$ & minute & $2.77 E+03$ & $7.9883 E+07$ & $1.2518 \mathrm{E}-08$ & & & \\
\hline Mn 52 & 51.9456 & $5: 591 E+00$ & day & $4.83 E+05$ & $4.4960 \mathrm{E}+05$ & $2.2242 \mathrm{E}-06$ & & & \\
\hline$M n 52 m$ & 51.9456 & $2.110 E+01$ & minute & $1.27 \mathrm{E}+03$ & $1.7155 E+08$ & $5.8292 \mathrm{E}-09$ & & & \\
\hline $\mathrm{Mn} 53$ & 52.9413 & $3.740 E+06$ & year & $1.18 \mathrm{E}+14$ & $1.8055 \mathrm{E}-03$ & $5.5387 \mathrm{E}+02$ & & & \\
\hline Mn 54 & 53.9404 & $3.123 \mathrm{E}+02$ & day & $2.70 \mathrm{E}+07$ & $7.7513 E+03$ & $1.2901 \mathrm{E}-04$ & 0.004975 & & \\
\hline $\mathrm{Mn} 56$ & 55.9389 & $2.579 E+00$ & hour & $9.28 \mathrm{E}+03$ & $2.1727 \mathrm{E}+07$ & $4.6026 \mathrm{E}-08$ & & & \\
\hline Mn 57 & 56.9383 & $8.540 \mathrm{E}+01$ & second & $8.54 \mathrm{E}+01$ & $2.3201 \mathrm{E}+09$ & $4.3101 \mathrm{E}-10$ & & & \\
\hline $\mathrm{Mn} 58$ & 57.9400 & $3.000 E+00$ & second & $3.00 E+00$ & $2.9819 \mathrm{E}+07$ & $3.3535 E-08$ & & & \\
\hline Mn 58m & 57.9400 & $1.088 \mathrm{E}+02$ & minute & $6.53 E+03$ & $6.4905 \mathrm{E}+10$ & $1.5407 \mathrm{E}-11$ & & & \\
\hline Mn 59 & 58.9404 & $4.600 E+00$ & second & $4.60 E+00$ & $4.1611 \mathrm{E}+10$ & $2.4032 \mathrm{E}-11$ & & & \\
\hline $\operatorname{Mn} 60$ & 59.9432 & $5.100 E+01$ & second & $5.10 \mathrm{E}+01$ & $3.6903 E+09$ & $2.7098 \mathrm{E}-10$ & & & \\
\hline $\mathrm{Mn} 60 \mathrm{~m}$ & 59.9432 & $1.770 E+00$ & second & $1.77 E+00$ & $1.0633 E+11$ & $9.4045 \mathrm{E}-12$ & & & \\
\hline $\mathrm{Mn} 62$ & 61.9480 & $8.800 \mathrm{E}-01$ & second & 8.80 E-01 & $2.0695 E+11$ & $4.8321 \mathrm{E}-12$ & & & \\
\hline $\mathrm{Fe} 49$ & 48.9736 & $7.500 \mathrm{E}-02$ & second & $7.50 \mathrm{E}-02$ & $3.0715 \mathrm{E}+12$ & $3.2557 \mathrm{E}-13$ & & & \\
\hline Fe 51 & 50.9568 & $3.050 \mathrm{E}-01$ & second & 3.05E-01 & $7.2590 E+11$ & $1.3776 \mathrm{E}-12$ & & & \\
\hline $\mathrm{Fe} 52$ & 51.9481 & $8.275 E+00$ & hour & $2.98 E+04$ & $7.2901 \mathrm{E}+06$ & 1.3717E-07 & & & \\
\hline Fe $52 m$ & 51.9481 & $4.590 E+01$ & second & $4.59 \mathrm{E}+01$ & $4.7314 E+09$ & $2.1135 \mathrm{E}-10$ & & & \\
\hline $\mathrm{Fe} \mathrm{53}$ & 52.9453 & $8.510 E+00$ & minute & $5.11 E+02$ & $4.1732 E+08$ & $2.3962 \mathrm{E}-09$ & & & \\
\hline $\mathrm{Fe} 53 \mathrm{~m}$ & 52.9453 & $2.580 E+00$ & minute & $1.55 \mathrm{E}+02$ & $1.3765 E+09$ & $7.2648 \mathrm{E}-10$ & & & \\
\hline $\mathrm{Fe} 55$ & 54.9383 & $2.730 E+00$ & year & $8.62 \mathrm{E}+07$ & $2.3835 \mathrm{E}+03$ & 4.1954E-04 & 0.000032 & & \\
\hline Fe 59 & 58.9349 & $4.450 E+01$ & day & $3.85 \mathrm{E}+06$ & $4.9785 E+04$ & $2.0086 \mathrm{E}-05$ & 0.007741 & & \\
\hline $\mathrm{Fe} 60$ & 59.9341 & $1.500 \mathrm{E}+06$ & year & $4.73 \mathrm{E}+13$ & $3.9765 \mathrm{E}-03$ & $2.5148 E+02$ & & & \\
\hline $\mathrm{Fe} 61$ & 60.9367 & $5.980 \mathrm{E}+00$ & minute & $3.59 \mathrm{E}+02$ & $5.1599 E+08$ & 1.9380 E-09 & & & \\
\hline $\mathrm{Fe} 62$ & 61.9368 & $6.800 E+01$ & second & $6.80 \mathrm{E}+01$ & $2.6787 E+09$ & $3.7332 \mathrm{E}-10$ & & & \\
\hline $\mathrm{Fe} 63$ & 62.9401 & $6.100 E+00$ & second & $6.10 E+00$ & $2.9385 \mathrm{E}+10$ & $3.4031 \mathrm{E}-11$ & & & \\
\hline Co 53 & 52.9542 & $2.400 \mathrm{E}-01$ & second & $2.40 \mathrm{E}-01$ & $8.8770 \mathrm{E}+11$ & $1.1265 \mathrm{E}-12$ & & & \\
\hline Co 53m & 52.9542 & $2.470 \mathrm{E}-01$ & second & $2.47 \mathrm{E}-01$ & $8.6254 \mathrm{E}+11$ & 1.1594E-12 & & & \\
\hline
\end{tabular}

WSRC-TR-99-00344

October, 1999

page 9 


\begin{tabular}{|c|c|c|c|c|c|c|c|c|c|}
\hline Isotope & $\begin{array}{l}\text { Atomic } \\
\text { Mass }\end{array}$ & Halflife & $\begin{array}{l}\text { Time } \\
\text { Units }\end{array}$ & $t_{1 / 2}, \sec$ & Curies/gram & Grams/Curie & $\begin{array}{c}\text { Watts/Cl } \\
\text { (Ref.3) }\end{array}$ & $\begin{array}{c}\text { Individual' }^{1} \\
\text { Fission } \\
\text { Product Yield } \\
\text { (Ref.4) }\end{array}$ & $\begin{array}{l}\text { Cumulative } \\
\text { Fission Product } \\
\text { Yleld (Ref.4) }\end{array}$ \\
\hline Co 54 & 53.9485 & $1.933 \mathrm{E}-01$ & second & $1.93 E-01$ & $1.0819 E+12$ & $9.2430 \mathrm{E}-13$ & & & \\
\hline Co $54 m$ & 53.9485 & $1.480 \mathrm{E}+00$ & minute & $8.88 \mathrm{E}+01$ & $2.3550 \mathrm{E}+09$ & $4.2463 \mathrm{E}-10$ & & & \\
\hline Co55 & 54.9420 & $1.753 E+01$ & hour & $6.31 E+04$ & $3.2538 E+06$ & $3.0734 \mathrm{E}-07$ & & & \\
\hline Co 56 & 55.9398 & $7.727 \mathrm{E}+01$ & day & $6.68 E+06$ & $3.0209 E+04$ & $3.3103 \mathrm{E}-05$ & & & \\
\hline Co 57 & 56.9363 & $2.718 E+02$ & day & $2.35 E+07$ & $8.4380 E+03$ & 1.1851E-04 & 0.846400 & & \\
\hline Co 58 & $57: 9358$ & $7.082 E+01$ & day & $6.12 \mathrm{E}+06$ & $3.1824 E+04$ & $3.1422 \mathrm{E}-05$ & 0.005990 & & \\
\hline Co $58 \mathrm{~m}$ & 57.9358 & $9.150 E+00$ & hour & $3.29 E+04$ & $5.9116 E+06$ & 1.6916E-07 & & & \\
\hline 0060 & 59.9338 & $5.271 \mathrm{E}+00$ & year & $1.66 E+08$ & $1.1315 E+03$ & $8.8377 \mathrm{E}-04$ & 0.015410 & & \\
\hline $\mathrm{Co} 60 \mathrm{~m}$ & 59.9338 & $1.047 \mathrm{E}+01$ & minute & $6.28 \mathrm{E}+02$ & $2.9964 \mathrm{E}+08$ & $3.3373 \mathrm{E}-09$ & 0.000357 & & \\
\hline $\operatorname{Co61}$ & 60.9325 & $1.650 E+00$ & hour & $5.94 E+03$ & $3.1170 E+07$ & $3.2082 E-08$ & & & \\
\hline Co 62 & 61.9341 & $1.500 E+00$ & minute & $9.00 \mathrm{E}+01$ & $2.0240 E+09$ & $4.9408 \mathrm{E}-10$ & & & \\
\hline Co $62 \mathrm{~m}$. & 61.9341 & $1.391 \mathrm{E}+01$ & minute & $8.35 E+02$ & $2.1826 E+08$ & $4.5818 \mathrm{E}-09$ & & & \\
\hline 0063 & 62.9336 & $2.740 E+01$ & second & $2.74 E+01$ & $6.5425 E+09$ & $1.5285 \mathrm{E}-10$ & & & \\
\hline Co64 & 63.9358 & $3.000 \mathrm{E}-01$ & second & $3.00 \mathrm{E}-01$ & $5.8818 \mathrm{E}+11$ & $1.7002 \mathrm{E}-12$ & & & \\
\hline Ni 53 & 52.9685 & $4.500 \mathrm{E}-02$ & second & $4.50 \mathrm{E}-02$ & $4.7331 E+12$ & $2.1128 \mathrm{E}-13$ & & & \\
\hline Ni 55 & 54.9513 & $2.121 E-01$ & second & $2.12 \mathrm{E}-01$ & $9.6796 \mathrm{E}+11$ & $1.0331 \mathrm{E}-12$ & & & \\
\hline Ni 56 & 55.9421 & $5.900 E+00$ & day & $5.10 \mathrm{E}+05$ & $3.9561 E+05$ & $2.5277 \mathrm{E}-06$ & & & \\
\hline Ni 57 & 56.9398 & $1.483 E+00$ & day & $1.28 \mathrm{E}+05$ & $1.5460 E+06$ & $6.4682 \mathrm{E}-07$ & & & \\
\hline Ni 59 & 58.9344 & $7.600 E+04$ & year & $2.40 \mathrm{E}+12$ & $7.9814 \mathrm{E}-02$ & $1.2529 \mathrm{E}+01$ & 0.000040 & & \\
\hline $\mathrm{Ni} 63$ & 62.9297 & $1.001 E+02$ & year & $3.16 \mathrm{E}+09$ & $5.6751 \mathrm{E}+01$ & 1.7621E-02 & 0.000101 & & \\
\hline $\mathrm{Ni} 65$ & 64.9301 & $2.517 \mathrm{E}+00$ & hour & $9.06 \mathrm{E}+03$ & $1.9174 E+07$ & $5.2154 \mathrm{E}-08$ & & & \\
\hline $\mathrm{Ni} 66$ & 65.9291 & $2.275 E+00$ & day & $1.97 \mathrm{E}+05$ & $8.7057 E+05$ & $1.1487 E-06$ & & $2.129 \mathrm{E}-11$ & $7.622 \mathrm{E}-10$ \\
\hline Ni 67 & 66.9316 & $2.100 E+01$ & second & $2.10 \mathrm{E}+01$ & $8.0265 \mathrm{E}+09$ & $1.2459 \mathrm{E}-10$ & & $6.029 E-10$ & $3.908 \mathrm{E}-09$ \\
\hline Cu 58 & 57.9445 & $3.204 E+00$ & second & $3.20 E+00$ & $6.0768 \mathrm{E}+10$ & $1.6456 \mathrm{E}-11$ & & & \\
\hline Cu 59 & 58.9395 & $1.358 \mathrm{E}+00$ & minute & $8.15 E+01$ & $2.3492 E+09$ & $4.2568 \mathrm{E}-10$ & & & \\
\hline $\mathrm{Cu} 60$ & 59.9374 & $2.370 \mathrm{E}+01$ & minute & $1.42 E+03$ & $1.3237 E+08$ & $7.5548 \mathrm{E}-09$ & & & \\
\hline Cu 61 & 60.9335 & $3.333 \mathrm{E}+00$ & hour & $1.20 \mathrm{E}+04$ & $1.5431 \mathrm{E}+07$ & $6.4806 \mathrm{E}-08$ & & & \\
\hline Cu 62 & 61.9326 & $9.740 E+00$ & minute & $5.84 E+02$ & $3.1171 \mathrm{E}+08$ & $3.2081 \mathrm{E}-09$ & & & \\
\hline Cu 64 & 63.9298 & $1.270 E+01$ & hour & $4.57 \mathrm{E}+04$ & $3.8598 \mathrm{E}+06$ & $2.5908 \mathrm{E}-07$ & & & \\
\hline Cu 66 & 65.9289 & $5.088 E+00$ & minute & $3.05 E+02$ & $5.6053 E+08$ & $1.7840 \mathrm{E}-09$ & & $7.808 E-14$ & $7.623 \mathrm{E}-10$ \\
\hline Cu 67 & 66.9278 & $2.576 E+00$ & day & $2.23 \mathrm{E}+05$ & $7.5730 E+05$ & $1.3205 \mathrm{E}-06$ & & $5.619 E-12$ & $3.913 E-09$ \\
\hline Cu 68 & 67.9296 & $3.110 E+01$ & second & $3.11 \mathrm{E}+01$ & $5.3402 \mathrm{E}+09$ & $1.8726 \mathrm{E}-10$ & & $1.921 \mathrm{E}-11$ & $6.598 E-09$ \\
\hline $\mathrm{Cu} 68 \mathrm{~m}$ & 67.9296 & $3.750 \mathrm{E}+00$ & minute & $2.25 \mathrm{E}+02$ & $7.3813 E+08$ & $1.3548 \mathrm{E}-09$ & & $4.498 \mathrm{E}-11$ & $4.498 \mathrm{E}-11$ \\
\hline Cu 69 & 68.9294 & $2.850 E+00$ & minute & $1.71 \mathrm{E}+02$ & $9.5714 \mathrm{E}+08$ & $1.0448 \mathrm{E}-09$ & & $7.028 \mathrm{E}-10$ & $1.432 \mathrm{E}-08$ \\
\hline Cu 70 & 69.9324 & $4.500 E+00$ & second & $4.50 \mathrm{E}+00$ & $3.5850 E+10$ & $2.7894 \mathrm{E}-11$ & & $1.090 \mathrm{E}-09$ & $2.962 E-08$ \\
\hline
\end{tabular}

WSRC-TR-99-00344

October, 1999

page 10 


\begin{tabular}{|c|c|c|c|c|c|c|c|c|c|}
\hline Isotope & $\begin{array}{l}\text { Atomic } \\
\text { Mass }\end{array}$ & Halflife & $\begin{array}{l}\text { Time } \\
\text { Units }\end{array}$ & $t_{1 / 2}, \sec$ & Curies/ gram & Grams/Curie & $\begin{array}{c}\text { Watts/Ci } \\
\text { (Ref.3) }\end{array}$ & $\begin{array}{c}\text { Individual }^{1} \\
\text { Fission } \\
\text { Product Yield } \\
\text { (Ref.4) }\end{array}$ & $\begin{array}{l}\text { Cumulative }^{4} \\
\text { Fission Product } \\
\text { Yield (Ref.4) }\end{array}$ \\
\hline $\mathrm{Cu} 70 \mathrm{~m}$ & 69.9324 & $4.700 \mathrm{E}+01$ & second & $4.70 E+01$ & $3.4324 E+09$ & $2.9134 \mathrm{E}-10$ & & $3.289 \mathrm{E}-09$ & $3.289 \mathrm{E}-09$ \\
\hline $\mathrm{Cu} 71$ & 70.9326 & $1.950 \mathrm{E}+01$ & second & $1.95 \mathrm{E}+01$ & $8.1564 E+09$ & $1.2260 \mathrm{E}-10$ & & $2.579 \mathrm{E}-08$ & $7.473 E-08$ \\
\hline $\mathrm{Cu} 72$ & 71.9355 & $6.600 \mathrm{E}+00$ & second & $6.60 \mathrm{E}+00$ & $2.3762 E+10$ & $4.2083 \mathrm{E}-11$ & & $1.249 \mathrm{E}-07$ & $2.290 E-07$ \\
\hline $\mathrm{Cu} 73$ & 72.9365 & $3.900 \mathrm{E}+00$ & second & $3.90 E+00$ & $3.9661 \mathrm{E}+10$ & $2.5213 \mathrm{E}-11$ & & $5.999 \mathrm{E}-07$ & $7.540 \mathrm{E}-07$ \\
\hline$\widehat{C u ~} 74$ & 73.9402 & $1.594 \mathrm{E}+00$ & second & $1.59 E+00$ & $9.5721 \mathrm{E}+10$ & $1.0447 \mathrm{E}-11$ & & $1.060 \mathrm{E}-06$ & $1.177 \mathrm{E}-06$ \\
\hline Cu 75 & 74.9417 & $1.224 \mathrm{E}+00$ & second & $1.22 E+00$ & $1.2299 \mathrm{E}+11$ & $8.1307 \mathrm{E}-12$ & & $1.760 \mathrm{E}-06$ & $1.815 \mathrm{E}-06$ \\
\hline $2 n 57$ & 56.9649 & $4.000 \mathrm{E}-02$ & second & $4.00 \mathrm{E}-02$ & $4.9512 E+12$ & $2.0197 \mathrm{E}-13$ & & & \\
\hline $\operatorname{Zn} 59$ & 58.9493 & $1.820 \mathrm{E}-01$ & second & $1.82 \mathrm{E}-01$ & $1.0515 E+12$ & $9.5098 \mathrm{E}-13$ & & & \\
\hline $2 \ln 60$ & 59.9418 & $2.380 \mathrm{E}+00$ & minute & $1.43 E+02$ & $1.3180 E+09$ & $7.5872 \mathrm{E}-10$ & & & \\
\hline $\operatorname{Zn} 61$ & 60.9395 & $1.485 \mathrm{E}+00$ & minute & $8.91 E+01$ & $2.0778 \mathrm{E}+09$ & $4.8128 \mathrm{E}-10$ & & & \\
\hline $\operatorname{Zn} 62$ & 61.9343 & $9.186 E+00$ & hour & $3.31 E+04$ & $5.5083 \mathrm{E}+06$ & $1.8154 \mathrm{E}-07$ & & & \\
\hline $2 n 63$ & 62.9332 & $3.847 E+01$ & minute & $2.31 E+03$ & $7.7665 \mathrm{E}+07$ & $1.2876 \mathrm{E}-08$ & & & \\
\hline $2 n 65$ & 64.9292 & $2.443 E+02$ & day & $2.11 E+07$ & $8.2332 E+03$ & $1.2146 \mathrm{E}-04$ & 0.0 & & \\
\hline $2 n 69$ & 68.9266 & $5.640 \mathrm{E}+01$ & minute & $3.38 E+03$ & $4.8368 E+07$ & $2.0675 \mathrm{E}-08$ & & $1.375 \mathrm{E}-12$ & $1.433 E-08$ \\
\hline $2 n 69 m$ & 68.9266 & $1.376 \mathrm{E}+01$ & hour & $4.95 E+04$ & $3.3042 E+06$ & $3.0264 \mathrm{E}-07$ & & $5.944 \mathrm{E}-12$ & $5.9439 d-12$ \\
\hline $\operatorname{Zn~} 71$ & 70.9277 & $2.450 E+00$ & minute & $1.47 \mathrm{E}+02$ & $1.0820 E+09$ & $9.2418 \mathrm{E}-10$ & & $4.497 \mathrm{E}-10$ & $6.809 \mathrm{E}-08$ \\
\hline $2 \mathrm{n} 71 \mathrm{~m}$ & 70.9277 & $3.960 \mathrm{E}+00$ & hour & $1.43 E+04$ & $1.1157 E+07$ & $8.9627 \mathrm{E}-08$ & & $1.945 \mathrm{E}-09$ & $9.044 \mathrm{E}-09$ \\
\hline $\operatorname{Zn~} 72$ & 71.9269 & $1.938 \mathrm{E}+00$ & day & $1.67 \mathrm{E}+05$ & $9.3698 E+05$ & $1.0673 \mathrm{E}-06$ & & $3.889 \mathrm{E}-08$ & $2.679 \mathrm{E}-07$ \\
\hline $\operatorname{Zn} 73$ & 72.9298 & $2.350 \mathrm{E}+01$ & second & $2.35 E+01$ & $6.5827 E+09$ & $1.5191 \mathrm{E}-10$ & & $4.139 \mathrm{E}-07$ & $1.168 \mathrm{E}-06$ \\
\hline $2 n 73 m$ & 72.9298 & $5.800 \mathrm{E}+00$ & second & $5.80 \mathrm{E}+00$ & $2.6671 \mathrm{E}+10$ & $3.7494 \mathrm{E}-11$ & & & \\
\hline $2 n 74$ & 73.9295 & $1.600 \mathrm{E}+00$ & minute & $9.60 \mathrm{E}+01$ & $1.5896 E+09$ & $6.2909 \mathrm{E}-10$ & & $2.310 \mathrm{E}-06$ & $3.550 \mathrm{E}-06$ \\
\hline $\operatorname{Zn~} 75$ & 74.9329 & $1.020 \mathrm{E}+01$ & second & $1.02 E+01$ & $1.4761 \mathrm{E}+10$ & $6.7748 \mathrm{E}-11$ & & $8.348 \mathrm{E}-06$ & $1.015 \mathrm{E}-05$ \\
\hline $2 n 76$ & 75.9334 & $5.700 \mathrm{E}+00$ & second & $5.70 \mathrm{E}+00$ & $2.6066 \mathrm{E}+10$ & $3.8365 \mathrm{E}-11$ & & $2.538 \mathrm{E}-05$ & $2.699 \mathrm{E}-05$ \\
\hline $\operatorname{Zn} 77$ & 76.9371 & $2.080 E+00$ & second & $2.08 E+00$ & $7.0498 E+10$ & $1.4185 \mathrm{E}-11$ & & $3.059 \mathrm{E}-05$ & $3.141 \mathrm{E}-05$ \\
\hline $\mathrm{Zn} \mathrm{77m}$ & 76.9371 & $1.050 \mathrm{E}+00$ & second & $1.05 E+00$ & $1.3965 \mathrm{E}+11$ & $7.1606 \mathrm{E}-12$ & & & \\
\hline $2 n 78$ & 77.9386 & $1.470 E+00$ & second & $1.47 E+00$ & $9.8471 E+10$ & $1.0155 \mathrm{E}-11$ & & & \\
\hline Ga 62 & 61.9442 & $1.161 \mathrm{E}-01$ & second & $1.16 \mathrm{E}-01$ & $1.5684 E+12$ & $6.3757 \mathrm{E}-13$ & & & \\
\hline$\overline{\mathrm{Ga} 63}$ & 62.9391 & $3.240 E+01$ & second & $3.24 E+01$ & $5.5324 E+09$ & $1.8075 \mathrm{E}-10$ & & & \\
\hline Ga 64 & 63.9368 & $2.630 \mathrm{E}+00$ & minute & $1.58 \mathrm{E}+02$ & $1.1182 \mathrm{E}+09$ & $8.9430 \mathrm{E}-10$ & & & \\
\hline Ga 65 & 64.9327 & $1.520 E+01$ & minute & $9.12 E+02$ & $1.9051 \mathrm{E}+08$ & $5.2491 \mathrm{E}-09$ & & & \\
\hline Ga 66 & 65.9316 & $9.490 \mathrm{E}+00$ & hour & $3.42 E+04$ & $5.0086 \mathrm{E}+06$ & $1.9966 \mathrm{E}-07$ & & & \\
\hline Ga 67 & 66.9282 & $3.261 E+00$ & day & $2.82 E+05$ & $5.9824 \mathrm{E}+05$ & $1.6716 \mathrm{E}-06$ & 0.001115 & & \\
\hline Ga 68 & 67.9280 & $1.127 E+00$ & hour & $4.06 E+03$ & $4.0930 E+07$ & $2.4432 \mathrm{E}-08$ & & $3.762 \mathrm{E}-18$ & $3.762 \mathrm{E}-18$ \\
\hline Ga 70 & 69.9260 & $2.114 \mathrm{E}+01$ & minute & $1.27 \mathrm{E}+03$ & $1.2720 \mathrm{E}+08$ & $7.8617 \mathrm{E}-09$ & & $1.130 \mathrm{E}-13$ & $1.130 \mathrm{E}-13$ \\
\hline Ga 72 & 71.9264 & $1.410 \mathrm{E}+01$ & hour & $5.08 E+04$ & $3.0901 E+06$ & $3.2362 E-07$ & & $2.609 \mathrm{E}-10$ & $2.681 \mathrm{E}-07$ \\
\hline
\end{tabular}

WSRC-TR-99-00344

October, 1999

page 11 


\begin{tabular}{|c|c|c|c|c|c|c|c|c|c|}
\hline Isotope & $\begin{array}{c}\text { Atomic } \\
\text { Mass }\end{array}$ & Halflife & $\begin{array}{l}\text { Time } \\
\text { Units }\end{array}$ & $t_{1 / 2}, \sec$ & Curies/ gram & Grams/ Curie & $\begin{array}{c}\text { Watts/Ci } \\
\text { (Ref.3) }\end{array}$ & $\begin{array}{c}\text { Individual }^{4} \\
\text { Fission } \\
\text { Product Yield } \\
\text { (Ref.4) }\end{array}$ & $\begin{array}{l}\text { Cumulative }^{1} \\
\text { Fission Product } \\
\text { Yield (Ref.4) }\end{array}$ \\
\hline Ga $72 m$ & 71.9264 & $3.970 E-02$ & second & $3.97 \mathrm{E}-02$ & $3.9509 E+12$ & $2.5311 \mathrm{E}-13$ & & & \\
\hline Ga 73 & 72.9252 & $4.860 \mathrm{E}+00$ & hour & $1.75 E+04$ & $8.8422 E+06$ & $1.1309 \mathrm{E}-07$ & & $9.998 \mathrm{E}-09$ & $1.178 \mathrm{E}-06$ \\
\hline Ga 74 & 73.9269 & $8.120 E+00$ & minute & $4.87 \mathrm{E}+02$ & $3.1323 E+08$ & $3.1925 \mathrm{E}-09$ & & $1.074 \mathrm{E}-07$ & $3.683 E-06$ \\
\hline Ga 74m & 73.9269 & $9.500 E+00$ & second & $9.50 E+00$ & $1.6064 \mathrm{E}+10$ & $6.2252 \mathrm{E}-11$ & & $2.555 E-08$ & $3.114 \mathrm{E}-06$ \\
\hline Ga 75 & 74.9265 & $2.100 E+00$ & minute & $1.26 E+02$ & $1.1950 \mathrm{E}+09$ & $8.3682 \mathrm{E}-10$ & & $1.580 E-06$ & 1.173E-05 \\
\hline Ga 76 & 75.9289 & $3.260 E+01$ & second & $3.26 E+01$ & $4.5578 \mathrm{E}+09$ & $2.1941 \mathrm{E}-10$ & & 1.051E-05 & 3.750 E-05 \\
\hline Ga 77 & 76.9293 & $1.320 E+01$ & second & $1.32 E+01$ & $1.1110 \mathrm{E}+10$ & $9.0010 E-11$ & & $4.077 E-05$ & $7.218 E-05$ \\
\hline Ga 78 & 77.9317 & $5.090 \mathrm{E}+00$ & second & $5.09 E+00$ & $2.8441 E+10$ & $3.5161 \mathrm{E}-11$ & & $1.077 \mathrm{E}-04$ & $1.428 \mathrm{E}-04$ \\
\hline Ga 79 & 78.9329 & $2.847 E+00$ & second & $2.85 E+00$ & $5.0203 E+10$ & $1.9919 \mathrm{E}-11$ & & $1.710 E-04$ & 1.877E-04 \\
\hline Ga 80 & 79.9366 & $1.697 E+00$ & second & $1.70 E+00$ & $8.3167 E+10$ & $1.2024 \mathrm{E}-11$ & & 1.057E-04 & $1.093 E-04$ \\
\hline Ga 81 & 80.9378 & $1.217 E+00$ & second & $1.22 \mathrm{E}+00$ & $1.1453 E+11$ & $8.7310 E-12$ & & 7.030 E-05 & $7.094 E-05$ \\
\hline Ga 82 & 81.9432 & $5.990 E-01$ & second & $5.99 \mathrm{E}-01$ & $2.2985 E+11$ & $4.3507 \mathrm{E}-12$ & & $6.885 E-05$ & $6.908 E-05$ \\
\hline Ga 83 & 82.9469 & $3.100 E-01$ & second & $3.10 \mathrm{E}-0.1$ & $4.3875 E+11$ & $2.2792 E-12$ & & $3.729 E-06$ & $3.732 E-06$ \\
\hline Ge 64 & 63.9416 & $1.062 E+00$ & minute & $6.37 E+01$ & $2.7697 \mathrm{E}+09$ & $3.6104 E-10$ & & & \\
\hline Ge 65 & 64.9394 & $3.090 E+01$ & second & $3.09 E+01$ & $5.6222 E+09$ & $1.7787 \mathrm{E}-10$ & & & \\
\hline Ge 66 & 65.9338 & $2.260 E+00$ & hour & $8.14 E+03$ & $2.1031 E+07$ & $4.7549 \mathrm{E}-08$ & & & \\
\hline Ge 67 & 66.9327 & $1.890 \mathrm{E}+01$ & minute & $1.13 E+03$ & $1.4864 E+08$ & $6.7278 \mathrm{E}-09$ & & & \\
\hline Ge 68 & 67.9281 & $2.708 \mathrm{E}+02$ & day & $2.34 \mathrm{E}+07$ & $7.0979 E+03$ & $1.4089 \mathrm{E}-04$ & & & \\
\hline $\mathrm{Ge} 69$ & 68.9280 & $1.627 \mathrm{E}+00$ & day & $1.41 \mathrm{E}+05$ & $1.1643 E+06$ & $8.5890 \mathrm{E}-07$ & & $8.006 \mathrm{E}-21$ & $8.005 \mathrm{E}-21$ \\
\hline Ge 71 & 70.9250 & $1.143 \mathrm{E}+01$ & day & $9.88 \mathrm{E}+05$ & $1.6107 \mathrm{E}+05$ & $6.2084 \mathrm{E}-06$ & & $4.056 \mathrm{E}-16$ & $4.056 \mathrm{E}-16$ \\
\hline Ge $71 m$ & 70.9250 & $2.040 E-02$ & second & $2.04 E-02$ & $7.7974 E+12$ & $1.2825 \mathrm{E}-13$ & & $5.353 E-12$ & $1.168 \mathrm{E}-06$ \\
\hline $\mathrm{Ge} 73 \mathrm{~m}$ & 72.9235 & $4.990 \mathrm{E}-01$ & second & 4.99E-01 & $3.1003 E+11$ & $3.2255 \mathrm{E}-12$ & & $2.315 \mathrm{E}-11$ & $1.178 \mathrm{E}-06$ \\
\hline Ge 75 & 74.9229 & $1.380 E+00$ & hour & $4.97 \mathrm{E}+03$ & $3.0316 E+07$ & $3.2986 \mathrm{E}-08$ & & $5.774 \mathrm{E}-09$ & 1.177E-05 \\
\hline Ge $75 m$ & 74.9229 & $4.770 E+01$ & second & $4.77 \mathrm{E}+01$ & $3.1568 \mathrm{E}+09$ & $3.1678 \mathrm{E}-10$ & & $3.778 \mathrm{E}-08$ & $5.421 \mathrm{E}-07$ \\
\hline Ge 77 & 76.9235 & $1.130 E+01$ & hour & $4.07 E+04$ & $3.6053 E+06$ & $2.7737 \mathrm{E}-07$ & & $1.045 \mathrm{E}-05$ & $2.602 E-05$ \\
\hline $\mathrm{Ge} 77 \mathrm{~m}$ & 76.9235 & $5.290 E+01$ & second & $5.29 \mathrm{E}+01$ & $2.7724 E+09$ & $3.6069 \mathrm{E}-10$ & & $1.597 \mathrm{E}-06$ & $7.378 \mathrm{E}=05$ \\
\hline Ge 78 & 77.9229 & $1.467 \mathrm{E}+00$ & hour & $5.28 E+03$ & $2.7421 E+07$ & $3.6469 \mathrm{E}-08$ & & $7.362 E-05$ & $2.166 E-04$ \\
\hline $\mathrm{Ge} 79$ & 78.9254 & $1.910 E+01$ & second & $1.91 \mathrm{E}+01$ & $7.4839 E+09$ & $1.3362 \mathrm{E}-10$ & & $3.148 \mathrm{E}-05$ & $2.190 \mathrm{E}-04$ \\
\hline Ge $79 m$ & 78.9254 & $3.900 E+01$ & second & $3.90 \mathrm{E}+01$ & $3.6652 \mathrm{E}+09$ & $2.7284 E-10$ & & $2.060 \mathrm{E}-04$ & $2.069 E-04$ \\
\hline $\mathrm{Ge} 80$ & 79.9252 & $2.950 \mathrm{E}+01$ & second & $2.95 \mathrm{E}+01$ & $4.7849 \mathrm{E}+09$ & $2.0899 \mathrm{E}-10$ & & $1.079 \mathrm{E}-03$ & $1.196 \mathrm{E}-03$ \\
\hline Ge 81 & 80.9288 & $7.600 \mathrm{E}+00$ & second & $7.60 E+00$ & $1.8343 E+10$ & $5.4518 \mathrm{E}-11$ & & $1.230 \mathrm{E}-03$ & $1.307 \mathrm{E}-03$ \\
\hline Ge $81 m$ & 80.9288 & $7.600 E+00$ & second & $7.60 \mathrm{E}+00$ & $1.8343 E+10$ & $5.4518 \mathrm{E}-11$ & & & \\
\hline Ge 82 & 81.9296 & $4.600 \mathrm{E}+00$ & second & $4.60 \mathrm{E}+00$ & $2.9935 E+10$ & $3.3406 \mathrm{E}-11$ & & $1.279 \mathrm{E}-03$ & $1.335 \mathrm{E}-03$ \\
\hline $\mathrm{Ge} 83$ & 82.9345 & $1.850 E+00$ & second & $1.85 \mathrm{E}+00$ & $7.3531 \mathrm{E}+10$ & $1.3600 \mathrm{E}-11$ & & $4.982 \mathrm{E}-04$ & $5.361 \mathrm{E}-04$ \\
\hline $\mathrm{Ge} 84$ & 83.9373 & $1.200 E+00$ & second & $1.20 \mathrm{E}+00$ & $1.1201 \mathrm{E}+11$ & $8.9281 \mathrm{E}-12$ & & $2.226 \mathrm{E}-04$ & $2.787 \mathrm{E}-04$ \\
\hline
\end{tabular}




\begin{tabular}{|c|c|c|c|c|c|c|c|c|c|}
\hline Isotope & $\begin{array}{c}\text { Atomic } \\
\text { Mass }\end{array}$ & Halflife & $\begin{array}{l}\text { Time } \\
\text { Units }\end{array}$ & $t_{1 / 2}, \sec$ & Curies/gram & Grams/ Curie & $\begin{array}{c}\text { Watts/Ci } \\
\text { (Ref.3) }\end{array}$ & $\begin{array}{c}\text { Individual }^{1} \\
\text { Fission } \\
\text { Product Yield } \\
\text { (Ref.4) }\end{array}$ & $\begin{array}{c}\text { Cumulative } \\
\text { Fission Product } \\
\text { Yield (Ref.4) }\end{array}$ \\
\hline As 67 & 66.9392 & $4.250 E+01$ & second & $4.25 E+01$ & $3.9656 E+09$ & $2.5217 \mathrm{E}-10$ & & & \\
\hline As 68 & 67.9368 & $2.527 \mathrm{E}+00$ & minute & $1.52 E+02$ & $1.0954 \mathrm{E}+09$ & $9.1289 \mathrm{E}-10$ & & & \\
\hline As 69 & 68.9323 & $1.520 \mathrm{E}+01$ & minute & $9.12 E+02$ & $1.7946 E+08$ & $5.5724 \mathrm{E}-09$ & & & \\
\hline As 70 & 69.9309 & $5.260 \mathrm{E}+01$ & minute & $3.16 E+03$ & $5.1118 \mathrm{E}+07$ & $1.9563 \mathrm{E}-08$ & & & \\
\hline As 71 & 70.9271 & $2.720 E+00$ & day & $2.35 E+05$ & $6.7683 E+05$ & $1.4775 \mathrm{E}-06$ & & & \\
\hline As 72 & 71.9268 & $1.083 E+00$ & day & $9.36 \mathrm{E}+04$ & $1.6758 \mathrm{E}+06$ & $5.9673 \mathrm{E}-07$ & & $8.330 \mathrm{E}-19$ & $8.330 \mathrm{E}-19$ \\
\hline As 73 & 72.9238 & $8.030 E+01$ & day & $6.94 E+06$ & $2.2299 \mathrm{E}+04$ & $4.4846 \mathrm{E}-05$ & & $4.212 \mathrm{E}-16$ & $4.212 \mathrm{E}-16$ \\
\hline As 74 & 73.9239 & $1.777 \mathrm{E}+01$ & day & $1.54 E+06$ & $9.9401 E+04$ & $1.0060 \mathrm{E}-05$ & & $2.555 \mathrm{E}-13$ & $2.555 \mathrm{E}-13$ \\
\hline As 76 & 75.9224 & $1.097 E+00$ & day & $9.48 E+04$ & $1.5682 \mathrm{E}+06$ & $6.3767 E-07$ & & 1.910E-09 & $1.910 \mathrm{E}-09$ \\
\hline As 77 & 76.9206 & $1.618 \mathrm{E}+00$ & day & $1.40 E+05$ & $1.0492 \mathrm{E}+06$ & $9.5309 \mathrm{E}-07$ & & $9.688 \mathrm{E}-08$ & 8.433E-05 \\
\hline As 78 & 77.9218 & $1.512 \mathrm{E}+00$ & hour & $5.44 E+03$ & $2.6604 \mathrm{E}+07$ & $3.7588 \mathrm{E}-08$ & & $1.620 \mathrm{E}-06$ & $2.182 \mathrm{E}-04$ \\
\hline As 79 & 78.9209 & $9.010 \mathrm{E}+00$ & minute & $5.41 E+02$ & $2.6443 E+08$ & $3.7817 \mathrm{E}-09$ & & $2.713 \mathrm{E}-05$ & $4.530 \mathrm{E}-04$ \\
\hline As 80 & 79.9226 & $1.520 \mathrm{E}+01$ & second & $1.52 \mathrm{E}+01$ & $9.2867 E+09$ & $1.0768 \mathrm{E}-10$ & & $1.109 \mathrm{E}-04$ & 1.307E-03 \\
\hline As 81 & 80.9221 & $3.330 \mathrm{E}+01$ & second & $3.33 E+01$ & $4.1866 \mathrm{E}+09$ & $2.3886 \mathrm{E}-10$ & & $5.653 \mathrm{E}-04$ & $1.873 \mathrm{E}-03$ \\
\hline As 82 & 81.9245 & $1.910 \mathrm{E}+01$ & second & $1.91 E+01$ & $7.2099 \mathrm{E}+09$ & $1.3870 \mathrm{E}-10$ & & $4.008 \mathrm{E}-04$ & $1.736 \mathrm{E}-03$ \\
\hline As $82 \mathrm{~m}$ & 81.9245 & $1.360 \mathrm{E}+01$ & second & $1.36 \mathrm{E}+01$ & $1.0126 \mathrm{E}+10$ & $9.8759 \mathrm{E}-11$ & & $1.209 \mathrm{E}-03$ & 1.209E-03 \\
\hline As 83 & 82.9250 & $1.340 \mathrm{E}+01$ & second & $1.34 E+01$ & $1.0153 \mathrm{E}+10$ & $9.8495 \mathrm{E}-11$ & & $2.960 \mathrm{E}-03$ & $3.509 \mathrm{E}-03$ \\
\hline As 84 & 83.9291 & $5.500 \mathrm{E}+00$ & second & $5.50 \mathrm{E}+00$ & $2.4440 \mathrm{E}+10$ & $4.0917 \mathrm{E}-11$ & & 5.435E-04 & $8.086 \mathrm{E}-04$ \\
\hline As $84 \mathrm{~m}$ & 83.9291 & $6.500 \mathrm{E}-01$ & second & $6.50 \mathrm{E}-01$ & $2.0680 \mathrm{E}+11$ & $4.8356 \mathrm{E}-12$ & & $1.640 \mathrm{E}-03$ & $1.645 \mathrm{E}-03$ \\
\hline As 85 & 84.9318 & $2.028 \mathrm{E}+00$ & second & $2.03 E+00$ & $6.5500 E+10$ & $1.5267 \mathrm{E}-11$ & & $1.521 \mathrm{E}-03$ & $1.560 \mathrm{E}-03$ \\
\hline As 86 & 85.9243 & $9.000 \mathrm{E}-01$ & second & $9.00 \mathrm{E}-01$ & $1.4589 \mathrm{E}+11$ & $6.8546 \mathrm{E}-12$ & & 8.925E-04 & $9.472 \mathrm{E}-04$ \\
\hline As 87 & 86.9396 & $4.800 \mathrm{E}-01$ & second & $4.80 \mathrm{E}-01$ & $2.7034 \mathrm{E}+11$ & $3.6990 \mathrm{E}-12$ & & $4.949 \mathrm{E}-04$ & $6.448 \mathrm{E}-04$ \\
\hline Se 68 & 67.9419 & $3.550 \mathrm{E}+01$ & second & $3.55 \mathrm{E}+01$ & $4.6775 E+09$ & $2.1379 \mathrm{E}-10$ & & & \\
\hline Se 69 & 68.9396 & $2.740 \mathrm{E}+01$ & second & $2.74 \mathrm{E}+01$ & $5.9725 \mathrm{E}+09$ & $1.6743 \mathrm{E}-10$ & & & \\
\hline Se 70 & 69.9335 & $4.110 \mathrm{E}+01$ & minute & $2.47 E+03$ & $6.5418 \mathrm{E}+07$ & $1.5286 \mathrm{E}-08$ & & & \\
\hline Se 71 & 70.9323 & $4.740 \mathrm{E}+00$ & minute & $2.84 E+02$ & $5.5925 E+08$ & $1.7881 \mathrm{E}-09$ & & & \\
\hline Se 72 & 71.9271 & $8.400 \mathrm{E}+00$ & day & $7.26 \mathrm{E}+05$ & $2.1612 E+05$ & $4.6271 \mathrm{E}-06$ & & & \\
\hline Se 73 & 72.9268 & $7.150 \mathrm{E}+00$ & hour & $2.57 E+04$ & $6.0101 \mathrm{E}+06$ & $1.6639 \mathrm{E}-07$ & & & \\
\hline Se $73 m$ & 72.9268 & $3.980 \mathrm{E}+01$ & minute & $2.39 E+03$ & $6.4782 E+07$ & $1.5436 E-08$ & & & \\
\hline $\mathrm{Se} 75$ & 74.9225 & $1.198 \mathrm{E}+02$ & day & $1.03 E+07$ & $1.4550 \mathrm{E}+04$ & $6.8727 \mathrm{E}-05$ & 0.002410 & $4.360 \mathrm{E}-16$ & $4.359 \mathrm{E}-16$ \\
\hline Se $77 \mathrm{~m}$ & 76.9199 & $1.736 \mathrm{E}+01$ & second & $1.74 E+01$ & $8.4487 \mathrm{E}+09$ & $1.1836 \mathrm{E}-10$ & & $1.044 \mathrm{E}-11$ & $8.433 \mathrm{E}-05$ \\
\hline Se 79 & 78.9185 & $6.500 E+04$ & year & $2.05 E+12$ & $6.9690 \mathrm{E}-02$ & $1.4349 \mathrm{E}+01$ & 0.000313 & 1.644E-07 & $4.532 \mathrm{E}-04$ \\
\hline Se $79 m$ & 78.9185 & $3.910 \mathrm{E}+00$ & minute & $2.35 \mathrm{E}+02$ & $6.0935 \mathrm{E}+08$ & $1.6411 \mathrm{E}-09$ & & $2.513 \mathrm{E}-08$ & $4.482 \mathrm{E}-04$ \\
\hline Se 81 & 80.9180 & $1.845 \mathrm{E}+01$ & minute & $1.11 \mathrm{E}+03$ & $1.2595 E+08$ & 7.9399E-09 & & $1.050 \mathrm{E}-05$ & 1.952E-03 \\
\hline Se $81 \mathrm{~m}$ & 80.9180 & $5.728 \mathrm{E}+01$ & minute & $3.44 \mathrm{E}+03$ & $4.0567 \mathrm{E}+07$ & $2.4650 \mathrm{E}-08$ & & $6.867 \mathrm{E}-05$ & $9.320 \mathrm{E}-05$ \\
\hline
\end{tabular}

WSRC-TR-99-00344

October, 1999

page 13 


\begin{tabular}{|c|c|c|c|c|c|c|c|c|c|}
\hline Isotope & $\begin{array}{l}\text { Atomic } \\
\text { Mass }\end{array}$ & Halflife & $\begin{array}{l}\text { Time } \\
\text { Units }\end{array}$ & $t_{1 / 2}, \sec$ & Curies/ gram & Grams/ Curle & $\begin{array}{c}\text { Watts/CI } \\
\text { (Ref.3) }\end{array}$ & $\begin{array}{c}\text { Individual }^{1} \\
\text { Fission } \\
\text { Product Yield } \\
\text { (Ref.4) } \\
\end{array}$ & $\begin{array}{c}\text { Cumulative' } \\
\text { Fission Product } \\
\text { Yield (Ref.4) }\end{array}$ \\
\hline Se 83 & 82.9191 & $2.230 E+01$ & minute & $1.34 \mathrm{E}+03$ & $1.0169 E+08$ & $9.8341 \mathrm{E}-09$ & & $1.363 \mathrm{E}-03$ & $2.170 \mathrm{E}-03$ \\
\hline Se $83 m$ & 82.9191 & $1.168 \mathrm{E}+00$ & minute & $7.01 E+01$ & $1.9410 \mathrm{E}+09$ & $5.1521 E-10$ & & $3.152 \mathrm{E}-04$ & $3.018 \mathrm{E}-03$ \\
\hline Se 84 & 83.9185 & $3.100 E+00$ & minute & $1.86 \mathrm{E}+02$ & $7.2278 \mathrm{E}+08$ & $1.3835 \mathrm{E}-09$ & & $6.621 E-03$ & $9.854 \mathrm{E}-03$ \\
\hline Se 85 & 84.9222 & $3.170 E+01$ & second & $3.17 E+01$ & $4.1908 \mathrm{E}+09$ & $2.3862 E-10$ & & $9.219 \mathrm{E}-03$ & $1.011 \mathrm{E}-02$ \\
\hline Se 86 & 85.9243 & $1.530 E+01$ & second & $1.53 \mathrm{E}+01$ & $8.5816 E+09$ & $1.1653 \mathrm{E}-10$ & & $1.179 \mathrm{E}-02$ & $1.290 \mathrm{E}-02$ \\
\hline Se 87 & 86.9285 & $5.850 \mathrm{E}+00$ & second & $5.85 \mathrm{E}+00$ & $2.2185 \mathrm{E}+10$ & $4.5076 \mathrm{E}-11$ & & $7.120 \mathrm{E}-03$ & $7.591 \mathrm{E}-03$ \\
\hline Se 88 & 87.9314 & $1.520 \mathrm{E}+00$ & second & $1.52 \mathrm{E}+00$ & $8.4409 \mathrm{E}+10$ & $1.1847 \mathrm{E}-11$ & & $3.337 \mathrm{E}-03$ & $3.564 E-03$ \\
\hline Se 89 & 88.9360 & $4.100 \mathrm{E}-01$ & second & $4.10 E-01$ & $3.0940 \mathrm{E}+11$ & $3.2321 \mathrm{E}-12$ & & $1.203 E-03$ & $1.205 \mathrm{E}-03$ \\
\hline Se 91 & 90.9454 & $2.700 \mathrm{E}-01$ & second & $2.70 E-01$ & $4.5944 \mathrm{E}+11$ & $2.1765 \mathrm{E}-12$ & & $1.614 \mathrm{E}-05$ & $1.614 \mathrm{E}-05$ \\
\hline $\mathrm{Br} 72$ & 71.9365 & $1.310 E+00$ & minute & $7.86 \mathrm{E}+01$ & $1.9953 \mathrm{E}+09$ & $5.0118 E-10$ & & & \\
\hline $\mathrm{Br} 72 \mathrm{~m}$ & 71.9365 & $1.060 \mathrm{E}+01$ & second & $1.06 \mathrm{E}+01$ & $1.4795 E+10$ & $6.7589 \mathrm{E}-11$ & & & \\
\hline Br 73 & 72.9318 & $3.400 \mathrm{E}+00$ & minute & $2.04 E+02$ & $7.5828 \mathrm{E}+08$ & $1.3188 \mathrm{E}-09$ & & & \\
\hline $\mathrm{Br} 74$ & 73.9299 & $2.540 \mathrm{E}+01$ & minute & $1.52 E+03$ & $1.0013 \mathrm{E}+08$ & $9.9869 \mathrm{E}-09$ & & & \\
\hline $\mathrm{Br} 74 \mathrm{~m}$ & 73.9299 & $4.600 E+01$ & minute & $2.76 E+03$ & $5.5290 \mathrm{E}+07$ & $1.8086 \mathrm{E}-08$ & & & \\
\hline Br 75 & 74.9258 & $1.612 E+00$ & hour & $5.80 E+03$ & $2.5951 E+07$ & $3.8534 \mathrm{E}-08$ & & & \\
\hline $\mathrm{Br} 76$ & 75.9245 & $1.620 \mathrm{E}+01$ & hour & $5.83 E+04$ & $2.5479 E+06$ & $3.9249 \mathrm{E}-07$ & & & \\
\hline $\mathrm{Br} 76 \mathrm{~m}$ & 75.9245 & $1.310 E+00$ & second & $1.31 E+00$ & $1.1343 \mathrm{E}+11$ & $8.8161 \mathrm{E}-12$ & & & \\
\hline Br 77 & 76.9214 & $2.377 E+00$ & day & $2.05 E+05$ & $7.1430 \mathrm{E}+05$ & 1.4000 E- 06 & & & \\
\hline $\mathrm{Br} 77 \mathrm{~m}$ & 76.9214 & $4.280 E+00$ & minute. & $2.57 \mathrm{E}+02$ & $5.7113 \mathrm{E}+08$ & $1.7509 E-09$ & & & \\
\hline $\mathrm{Br} 78$ & 77.9211 & $6.460 \mathrm{E}+00$ & minute & $3.88 \mathrm{E}+02$ & $3.7354 \mathrm{E}+08$ & $2.6771 \mathrm{E}-09$ & & $2.190 \mathrm{E}-13$ & \\
\hline $\mathrm{Br} 79 \mathrm{~m}$ & 78.9183 & $4.860 E+00$ & second & $4.86 E+00$ & $2.9415 \mathrm{E}+10$ & $3.3997 E-11$ & & & \\
\hline $\mathrm{Br} 80$ & 79.9185 & $1.768 \mathrm{E}+01$ & minute & $1.06 E+03$ & $1.3307 \mathrm{E}+08$ & $7.5146 \mathrm{E}-09$ & & $1.944 \mathrm{E}-10$ & $7.808 \mathrm{E}-10$ \\
\hline $\mathrm{Br} 80 \mathrm{~m}$ & 79.9185 & $4.421 E+00$ & hour, & $1.59 E+04$ & $8.8706 \mathrm{E}+06$ & 1.1273E-07 & & $5.864 \mathrm{E}-10$ & $5.864 \mathrm{E}-10$ \\
\hline $\mathrm{Br} 82$ & 81.9168 & $1.471 \mathrm{E}+00$ & day & $1.27 E+05$ & $1.0837 \mathrm{E}+06$ & $9.2273 \mathrm{E}-07$ & & $4.309 \mathrm{E}-07$ & $6.104 \mathrm{E}-07$ \\
\hline $\mathrm{Br} 82 \mathrm{~m}$ & 81.9168 & $6.130 \mathrm{E}+00$ & minute & $3.68 \mathrm{E}+02$ & $3.7445 \mathrm{E}+08$ & $2.6706 E-09$ & & $1.840 \mathrm{E}-07$ & $1.840 E-07$ \\
\hline $\mathrm{Br} 83$ & 82.9152 & $2.400 \mathrm{E}+00$ & hour & $8.64 E+03$ & $1.5748 \mathrm{E}+07$ & $6.3500 \mathrm{E}-08$ & & $1.921 \mathrm{E}-04$ & $5.380 \mathrm{E}-03$ \\
\hline $\mathrm{Br} 84$ & 83.9165 & $3.180 E+01$ & minute & $1.91 E+03$ & $7.0461 \mathrm{E}+07$ & $1.4192 \mathrm{E}-08$ & & $1.768 \mathrm{E}-04$ & $1.003 E-02$ \\
\hline $\mathrm{Br} 84 \mathrm{~m}$ & 83.9165 & $6.000 \mathrm{E}+00$ & minute & $3.60 \mathrm{E}+02$ & $3.7344 \mathrm{E}+08$ & $2.6778 \mathrm{E}-09$ & & $3.181 \mathrm{E}-04$ & $3.181 \mathrm{E}-04$ \\
\hline $\mathrm{Br} 85$ & 84.9156 & $2.900 E+00$ & minute & $1.74 E+02$ & $7.6355 \mathrm{E}+08$ & $1.3097 \mathrm{E}-09$ & & $2.520 \mathrm{E}-03$ & $1.263 \mathrm{E}-02$ \\
\hline $\mathrm{Br} 86$ & 85.9188 & $5.510 \mathrm{E}+01$ & second & $5.51 \mathrm{E}+01$ & $2.3831 \mathrm{E}+09$ & $4.1963 \mathrm{E}-10$ & & $5.254 \mathrm{E}-03$ & $1.816 \mathrm{E}-02$ \\
\hline $\mathrm{Br} 87$ & 86.9207 & $5.560 \mathrm{E}+01$ & second & $5.56 \mathrm{E}+01$ & $2.3344 E+09$ & $4.2837 \mathrm{E}-10$ & & 1.257E-02 & $2.018 E-02$ \\
\hline $\mathrm{Br} 88$ & 87.9241 & $1.650 \mathrm{E}+01$ & second & $1.65 \mathrm{E}+01$ & $7.7765 E+09$ & $1.2859 \mathrm{E}-10$ & & $1.672 \mathrm{E}-02$ & $2.034 \mathrm{E}-02$ \\
\hline $\mathrm{Br} 89$ & 88.9264 & $4.400 \mathrm{E}+00$ & second & $4.40 E+00$ & $2.8833 E+10$ & $3.4682 E-11$ & & $1.206 \mathrm{E}-02$ & $1.319 E-02$ \\
\hline $\mathrm{Br} 90$ & 89.9306 & $1.910 \mathrm{E}+00$ & second & $1.91 \mathrm{E}+00$ & $6.5680 \mathrm{E}+10$ & $1.5225 \mathrm{E}-11$ & & $6.617 \mathrm{E}-03$ & $6.841 \mathrm{E}-03$ \\
\hline $\mathrm{Br} 91$ & 90.9340 & $5.410 \mathrm{E}-01$ & second & $5.41 E-01$ & $2.2933 \mathrm{E}+11$ & $4.3606 \mathrm{E}-12$ & & $2.446 \mathrm{E}-03$ & $2.458 \mathrm{E}-03$ \\
\hline
\end{tabular}




\begin{tabular}{|c|c|c|c|c|c|c|c|c|c|}
\hline Isotope & $\begin{array}{l}\text { Atomic } \\
\text { Mass }\end{array}$ & Halflife & $\begin{array}{l}\text { Time } \\
\text { Units }\end{array}$ & $t_{1 / 2}, \sec$ & Curies/gram & Grams/Curie & $\begin{array}{l}\text { Watts/Ci } \\
\text { (Ref.3) }\end{array}$ & $\begin{array}{c}\text { Individual }^{1} \\
\text { Fission } \\
\text { Product Yield } \\
\text { (Ref.4) } \\
\end{array}$ & $\begin{array}{l}\text { Cumulative } \\
\text { Fission Product } \\
\text { Yleld (Ref.4) }\end{array}$ \\
\hline $\mathrm{Br} 92$ & 91.9393 & $3.430 \mathrm{E}-01$ & second & $3.43 \mathrm{E}-01$ & $3.5775 E+11$ & $2.7952 E-12$ & & $3.605 \mathrm{E}-04$ & $3.614 \mathrm{E}-04$ \\
\hline $\mathrm{Kr} 72$ & 71.9419 & $1.720 \mathrm{E}+01$ & second & $1.72 \mathrm{E}+01$ & $9.1173 E+09$ & $1.0968 \mathrm{E}-10$ & & & \\
\hline $\mathrm{Kr} 73$ & 72.9389 & $2.700 E+01$ & second & $2.70 E+01$ & $5.7287 E+09$ & $1.7456 \mathrm{E}-10$ & & & \\
\hline Kr 74 & 73.9333 & $1.150 \mathrm{E}+01$ & minute & $6.90 \mathrm{E}+02$ & $2.2115 E+08$ & $4.5218 \mathrm{E}-09$ & & & \\
\hline $\mathrm{Kr} 75$ & 74.9310 & $4.300 \mathrm{E}+00$ & minute & $2.58 E+02$ & $5.8357 E+08$ & $1.7136 \mathrm{E}-09$ & & & \\
\hline $\mathrm{Kr} 76$ & 75.9259 & $1.480 E+01$ & hour & $5.33 E+04$ & $2.7888 E+06$ & 3.5857E-07 & & & \\
\hline $\mathrm{Kr} 77$ & 76.9247 & $1.240 \mathrm{E}+00$ & hour & $4.46 E+03$ & $3.2854 E+07$ & $3.0438 \mathrm{E}-08$ & & & \\
\hline $\mathrm{Kr} 79$ & 78.9201 & $1.460 \mathrm{E}+00$ & day & $1.26 E+05$ & $1.1332 E+06$ & $8.8242 E-07$ & & & \\
\hline $\mathrm{Kr} 79 \mathrm{~m}$ & 78.9201 & $5.000 E+01$ & second & $5.00 \mathrm{E}+01$ & $2.8590 E+09$ & $3.4977 \mathrm{E}-10$ & & & \\
\hline Kr 81 & 80.9166 & $2.290 E+05$ & year & $7.23 E+12$ & $1.9292 E-02$ & $5.1834 E+01$ & & $3.240 \mathrm{E}-11$ & $3.735 \mathrm{E}-11$ \\
\hline $\mathrm{Kr} 81 \mathrm{~m}$ & 80.9166 & $1.310 E+01$ & second & $1.31 E+01$ & $1.0643 E+10$ & $9.3958 \mathrm{E}-11$ & & $4.952 \mathrm{E}-12$ & $4.952 E-12$ \\
\hline $\mathrm{Kr} 83 \mathrm{~m}$ & 82.9141 & $1.830 E+00$ & hour & $6.59 E+03$ & $2.0654 E+07$ & $4.8418 \mathrm{E}-08$ & & 2.805E-08 & 5.379E-03 \\
\hline $\mathrm{Kr} 85$ & 84.9125 & $1.076 E+01$ & year & $3.39 \mathrm{E}+08$ & $3.9142 E+02$ & $2.5548 \mathrm{E}-03$ & 0.001500 & $5.664 E-05$ & $2.743 E-03$ \\
\hline $\mathrm{Kr} 85 \mathrm{~m}$ & 84.9125 & $4.480 E+00$ & hour & $1.61 E+04$ & $8.2380 E+06$ & $1.2139 \mathrm{E}-07$ & & $1.310 \mathrm{E}-05$ & $1.262 \mathrm{E}-02$ \\
\hline $\mathrm{Kr} 87$ & 86.9134 & $7.630 E+01$ & minute & $4.58 \mathrm{E}+03$ & $2.8354 E+07$ & $3.5268 \mathrm{E}-08$ & & $4.196 \mathrm{E}-03$ & $2.515 \mathrm{E}-02$ \\
\hline $\mathrm{Kr} 88$ & 87.9144 & $2.840 E+00$ & hour & $1.02 \mathrm{E}+04$ & $1.2551 \mathrm{E}+07$ & $7.9672 \mathrm{E}-08$ & & $1.475 \mathrm{E}-02$ & $3.568 \mathrm{E}-02$ \\
\hline $\mathrm{Kr} 89$ & 88.9176 & $3.150 \mathrm{E}+00$ & minute & $1.89 \mathrm{E}+02$ & $6.7132 E+08$ & $1.4896 \mathrm{E}-09$ & & $3.309 E-02$ & $4.611 \mathrm{E}-02$ \\
\hline $\mathrm{Kr} 90$ & 89.9195 & $3.232 \mathrm{E}+01$ & second & $3.23 E+01$ & $3.8820 E+09$ & $2.5760 \mathrm{E}-10$ & & $4.483 \mathrm{E}-02$ & $5.042 \mathrm{E}-02$ \\
\hline Kr 91 & 90.9234 & $8.570 E+00$ & second & $8.57 \mathrm{E}+00$ & $1.4478 E+10$ & $6.9069 \mathrm{E}-11$ & & $3.183 E-02$ & $3.396 \mathrm{E}-02$ \\
\hline $\mathrm{Kr} 92$ & 91.9262 & $1.840 \mathrm{E}+00$ & second & $1.84 E+00$ & $6.6699 E+10$ & $1.4993 \mathrm{E}-11$ & & $1.693 \mathrm{E}-02$ & $1.719 \mathrm{E}-02$ \\
\hline $\mathrm{Kr} 93$ & 92.9313 & $1.286 E+00$ & second & $1.29 \mathrm{E}+00$ & $9.4400 E+10$ & $1.0593 \mathrm{E}-11$ & & $5.245 \mathrm{E}-03$ & $5.266 \mathrm{E}-03$ \\
\hline $\mathrm{Kr} 94$ & 93.9344 & $2.000 \mathrm{E}-01$ & second & $2.00 E-01$ & $6.0051 E+11$ & $1.6652 \mathrm{E}-12$ & & $2.216 \mathrm{E}-03$ & $2.218 \mathrm{E}-03$ \\
\hline Kr95 & 94.9293 & $7.800 \mathrm{E}-01$ & second & $7.80 \mathrm{E}-01$ & $1.5236 E+11$ & $6.5632 \mathrm{E}-12$ & & $6.970 \mathrm{E}-05$ & $6.970 \mathrm{E}-05$ \\
\hline Kr97 & 96.9486 & $1.500 \mathrm{E}-07$ & second & $1.50 \mathrm{E}-07$ & $7.7579 E+17$ & $1.2890 \mathrm{E}-18$ & & $7.984 E-08$ & $7.984 \mathrm{E}-08$ \\
\hline $\mathrm{Rb} 75$ & 74.9386 & $1.900 \mathrm{E}+01$ & second & $1.90 \mathrm{E}+01$ & $7.9235 E+09$ & $1.2621 \mathrm{E}-10$ & & & \\
\hline $\mathrm{Rb} 76$ & 75.9351 & $3.650 \mathrm{E}+01$ & second & $3.65 \mathrm{E}+01$ & $4.0704 E+09$ & $2.4567 \mathrm{E}-10$ & & & \\
\hline $\mathrm{Rb} 77$ & 76.9304 & $3.750 \mathrm{E}+00$ & minute & $2.25 E+02$ & $6.5177 \mathrm{E}+08$ & $1.5343 \mathrm{E}-09$ & & & \\
\hline $\mathrm{Rb} 78$ & 77.9281 & $1.766 \mathrm{E}+01$ & minute & $1.06 \mathrm{E}+03$ & $1.3663 E+08$ & $7.3191 \mathrm{E}-09$ & & & \\
\hline $\mathrm{Rb} 78 \mathrm{~m}$ & 77.9281 & $5.740 \mathrm{E}+00$ & minute & $3.44 \mathrm{E}+02$ & $4.2036 E+08$ & $2.3789 \mathrm{E}-09$ & & & \\
\hline $\mathrm{Rb} 79$ & 78.9240 & $2.290 E+01$ & minute & $1.37 \mathrm{E}+03$ & $1.0404 E+08$ & $9.6121 \mathrm{E}-09$ & & & \\
\hline $\mathrm{Rb} 80$ & 79.9225 & $3.400 \mathrm{E}+01$ & second & $3.40 \mathrm{E}+01$ & $4.1517 \mathrm{E}+09$ & $2.4086 \mathrm{E}-10$ & & & \\
\hline $\mathrm{Rb} 81$ & 80.9190 & $4.576 \mathrm{E}+00$ & hour & $1.65 E+04$ & $8.4633 E+06$ & $1.1816 \mathrm{E}-07$ & & & \\
\hline $\mathrm{Rb} 81 \mathrm{~m}$ & 80.9190 & $3.050 \mathrm{E}+01$ & minute & $1.83 E+03$ & $7.6186 E+07$ & $1.3126 \mathrm{E}-08$ & & & \\
\hline $\mathrm{Rb} 82$ & 81.9182 & $1.273 \mathrm{E}+00$ & minute & $7.64 \mathrm{E}+01$ & $1.8031 E+09$ & $5.5460 \mathrm{E}-10$ & & & \\
\hline $\mathrm{Rb} 82 \mathrm{~m}$ & 81.9182 & $6.472 E+00$ & hour & $2.33 E+04$ & $5.9109 E+06$ & $1.6918 \mathrm{E}-07$ & & & \\
\hline
\end{tabular}




\begin{tabular}{|c|c|c|c|c|c|c|c|c|c|}
\hline Isotope & $\begin{array}{c}\text { Atomic } \\
\text { Mass }\end{array}$ & Halflife & $\begin{array}{l}\text { Time } \\
\text { Units }\end{array}$ & $t_{1 / 2}, \sec$ & Curies/gram & Grams/ Curie & $\begin{array}{c}\text { Watts/Ci } \\
\text { (Ref.3) }\end{array}$ & $\begin{array}{l}\text { Individual }^{1} \\
\text { Fission } \\
\text { Product Yield } \\
\text { (Ref.4) }\end{array}$ & $\begin{array}{c}\text { Cumulative }^{1} \\
\text { Fission Product } \\
\text { Yield (Ref.4) }\end{array}$ \\
\hline $\mathrm{Rb} 83$ & 82.9151 & $8.620 \mathrm{E}+01$ & day & $7.45 \mathrm{E}+06$ & $1.8269 E+04$ & $5.4737 \mathrm{E}-05$ & & & \\
\hline $\mathrm{Rb} 84$ & 83.9144 & $3.277 \mathrm{E}+01$ & day & $2.83 E+06$ & $4.7484 E+04$ & 2.1060E-05 & & & \\
\hline $\mathrm{Rb} 84 \mathrm{~m}$ & 83.9144 & $2.026 E+01$ & minute & $1.22 \mathrm{E}+03$ & $1.1060 E+08$ & 9.0417E-09 & & & \\
\hline $\mathrm{Rb} 86$ & 85.9112 & $1.863 \mathrm{E}+01$ & day & $1.61 \mathrm{E}+06$ & $8.1579 E+04$ & $1.2258 \mathrm{E}-05$ & 0.004520 & & \\
\hline $\mathrm{Rb} 86 \mathrm{~m}$ & 85.9112 & $1.017 \mathrm{E}+00$ & minute & $6.10 \mathrm{E}+01$ & $2.1521 E+09$ & $4.6467 E-10$ & & & \\
\hline $\mathrm{Rb} 87$ & 86.9092 & $4.750 E+10$ & year & $1.50 E+18$ & $8.6597 \mathrm{E}-08$ & $1.1548 E+07$ & & & \\
\hline $\mathrm{Rb} 88$ & 87.9113 & $1.778 \mathrm{E}+01$ & minute & $1.07 E+03$ & $1.2030 E+08$ & 8.3129E-09 & & $8.096 \mathrm{E}-04$ & $3.649 \mathrm{E}-02$ \\
\hline $\mathrm{Rb} 89$ & 88.9123 & $1.515 \mathrm{E}+01$ & minute & $9.09 E+02$ & $1.3959 \mathrm{E}+08$ & $7.1639 \mathrm{E}-09$ & & $2.396 \mathrm{E}-03$ & $4.851 E-02$ \\
\hline $\mathrm{Rb} 90$ & 89.9148 & $2.633 E+00$ & minute & $1.58 \mathrm{E}+02$ & $7.9413 E+08$ & $1.2592 E-09$ & & $1.589 \mathrm{E}-03$ & $4.641 \mathrm{E}-02$ \\
\hline $\mathrm{Rb} 90 \mathrm{~m}$ & 89.9148 & $4.300 \mathrm{E}+00$ & minute & $2.58 \mathrm{E}+02$ & $4.8632 E+08$ & 2.0562E-09 & & $6.681 \mathrm{E}-03$ & $1.283 E-02$ \\
\hline $\mathrm{Rb} 91$ & 90.9165 & $5.840 \mathrm{E}+01$ & second & $5.84 E+01$ & $2.1248 E+09$ & $4.7063 E-10$ & & $2.262 E-02$ & $5.658 \mathrm{E}-02$ \\
\hline $\mathrm{Rb} 92$ & 91.9197 & $4.492 E+00$ & second & $4.49 \mathrm{E}+00$ & $2.7323 E+10$ & $3.6599 \mathrm{E}-11$ & & $3.147 \mathrm{E}-02$ & $4.875 \mathrm{E}-02$ \\
\hline $\mathrm{Rb} 93$ & 92.9220 & $5.840 \mathrm{E}+00$ & second & $5.84 E+00$ & $2.0790 E+10$ & $4.8101 \mathrm{E}-11$ & & $3.084 \mathrm{E}-02$ & $3.613 E-02$ \\
\hline $\mathrm{Rb} 94$ & 93.9264 & $2.702 E+00$ & second & $2.70 \mathrm{E}+00$ & $4.4453 E+10$ & $2.2496 E-11$ & & $1.572 \mathrm{E}-02$ & $1.781 \mathrm{E}-02$ \\
\hline $\mathrm{Rb} 95$ & 94.9293 & $3.775 E-01$ & second & $3.78 \mathrm{E}-01$ & $3.1482 E+11$ & $3.1764 E-12$ & & $8.120 E-03$ & $8.183 E-03$ \\
\hline $\mathrm{Rb} 96$ & 95.9343 & 1.990E-01 & second & $1.99 \mathrm{E}-01$ & $5.9095 \mathrm{E}+11$ & $1.6922 \mathrm{E}-12$ & & $2.049 \mathrm{E}-03$ & $2.063 E-03$ \\
\hline $\mathrm{Rb} 97$ & 96.9373 & 1.699E-01 & second & $1.70 E-01$ & $6.8500 E+11$ & $1.4598 E-12$ & & $7.805 \mathrm{E}-04$ & $7.806 \mathrm{E}-04$ \\
\hline $\mathrm{Rb} 98$ & 97.9417 & 1.140E-01. & second & $1.14 \mathrm{E}-01$ & $1.0104 E+12$ & $9.8968 \mathrm{E}-13$ & & $2.444 \mathrm{E}-05$ & $2.445 E-05$ \\
\hline Rb $98 \mathrm{~m}^{\circ}$ & 97.9417 & $9.600 E-02$ & second & $9.60 \mathrm{E}-02$ & $1.1999 \mathrm{E}+12$ & $8.3342 E-13$ & & & \\
\hline $\mathrm{Rb} 99$ & 98.9454 & $5.030 E-02$ & second & $5.03 \mathrm{E}-02$ & $2.2668 \mathrm{E}+12$ & $4.4115 \mathrm{E}-13$ & & $3.953 \mathrm{E}-07$ & $3.953 \mathrm{E}-07$ \\
\hline $\mathrm{Rb} 100$ & 99.9499 & $5.100 E-02$ & second & $5.10 \mathrm{E}-02$ & $2.2132 E+12$ & $4.5183 E-13$ & & $7.668 \mathrm{E}-08$ & $7.669 \mathrm{E}-08$ \\
\hline $\mathrm{Rb} 101$ & 100.9532 & 3.200E-02 & second & $3.20 \mathrm{E}-02$ & $3.4923 E+12$ & $2.8635 \mathrm{E}-13$ & & $2.918 \mathrm{E}-10$ & $2.921 \mathrm{E}-10$ \\
\hline $\mathrm{Rb} 102$ & 101.9430 & $3.700 E-02$ & second & $3.70 \mathrm{E}-02$ & $2.9910 E+12$ & $3.3434 E-13$ & & $1.526 \mathrm{E}-12$ & $1.528 \mathrm{E}-12$ \\
\hline Sr 78 & 77.9322 & $2.500 \mathrm{E}+00$ & minute & $1.50 \mathrm{E}+02$ & $9.6509 E+08$ & $1.0362 E-09$ & & & \\
\hline Sr 79 & 78.9297 & $2.250 E+00$ & minute & $1.35 \mathrm{E}+02$ & $1.0588 \mathrm{E}+09$ & $9.4449 E-10$ & & & \\
\hline $\mathrm{Sr} 80$ & 79.9245 & $1.063 E+02$ & minute & $6.38 \mathrm{E}+03$ & $2.2132 E+07$ & 4.5184E-08 & & & \\
\hline Sr 81 & 80.9232 & $2.230 \mathrm{E}+01$ & minute & $1.34 \mathrm{E}+03$ & $1.0420 E+08$ & $9.5974 E-09$ & & & \\
\hline Sr 82 & 81.9184 & $2.555 E+01$ & day & $2.21 \mathrm{E}+06$ & $6.2386 \mathrm{E}+04$ & 1.6029E-05 & & & \\
\hline Sr 83 & 82.9176 & $1.350 \mathrm{E}+00$ & day & $1.17 \mathrm{E}+05$ & $1.1661 \mathrm{E}+06$ & $8.5752 E-07$ & & & \\
\hline $\mathrm{Sr} 83 \mathrm{~m}$ & 82.9176 & $4.950 \mathrm{E}+00$ & second & $4.95 E+00$ & $2.7487 E+10$ & $3.6381 \mathrm{E}-11$ & & & \\
\hline Sr 85 & 84.9129 & $6.484 E+01$ & day & $5.60 E+06$ & $2.3716 E+04$ & $4.2165 \mathrm{E}-05$ & & $3.938 \mathrm{E}-12$ & $4.732 \mathrm{E}-12$ \\
\hline $\mathrm{Sr} 85 \mathrm{~m}$ & 84.9129 & $1.127 \mathrm{E}+00$ & hour & $4.06 E+03$ & $3.2742 E+07$ & $3.0541 E-08$ & & $9.107 \mathrm{E}-13$ & $9.107 E-13$ \\
\hline $\mathrm{Sr} 87 \mathrm{~m}$ & 86.9089 & $2.803 E+00$ & hour & $1.01 \mathrm{E}+04$ & $1.2864 \mathrm{E}+07$ & $7.7734 E-08$ & & $3.599 \mathrm{E}-08$ & $2.554 \mathrm{E}-02$ \\
\hline Sr 89 & 88.9075 & $5.053 \mathrm{E}+01$ & day & $4.37 E+06$ & $2.9065 E+04$ & 3.4405E-05 & 0.003460 & $3.417 \mathrm{E}-04$ & $4.885 \mathrm{E}-02$ \\
\hline Sr 90 & 89.9077 & $2.878 \mathrm{E}+01$ & year & $9.08 \mathrm{E}+08$ & $1.3816 E+02$ & $7.2381 \mathrm{E}-03$ & 0.001160 & $3.563 E-04$ & $5.904 E-02$ \\
\hline
\end{tabular}

WSRC-TR-99-00344

October, 1999

page 16 


\begin{tabular}{|c|c|c|c|c|c|c|c|c|c|}
\hline Isotope & $\begin{array}{l}\text { Atomic } \\
\text { Mass }\end{array}$ & Halflife & $\begin{array}{l}\text { Time } \\
\text { Units }\end{array}$ & $t_{1 / 2}, \sec$ & Curies/gram & Grams/ Curie & $\begin{array}{c}\text { Watts/Ci } \\
\text { (Ref.3) }\end{array}$ & $\begin{array}{c}\text { Individual }^{1} \\
\text { Fission } \\
\text { Product Yield } \\
\text { (Ref.4) }\end{array}$ & $\begin{array}{l}\text { Cumulative }^{1} \\
\text { Fission Product } \\
\text { Yield (Ref.4) }\end{array}$ \\
\hline Sr 91 & 90.9102 & $9.630 \mathrm{E}+00$ & hour & $3.47 \mathrm{E}+04$ & $3.5796 \mathrm{E}+06$ & $2.7936 \mathrm{E}-07$ & & $2.592 \mathrm{E}-03$ & $5.918 \mathrm{E}-02$ \\
\hline $\mathrm{Sr} 92$ & 91.9110 & $2.710 \mathrm{E}+00$ & hour & $9.76 \mathrm{E}+03$ & $1.2582 \mathrm{E}+07$ & $7.9481 E-08$ & & $9.845 E-03$ & $5.908 \mathrm{E}-02$ \\
\hline Sr 93 & 92.9140 & $7.423 E+00$ & minute & $4.45 E+02$ & $2.7262 E+08$ & $3.6681 \mathrm{E}-09$ & & $2.508 \mathrm{E}-02$ & $6.254 \mathrm{E}-02$ \\
\hline Sr 94 & 93.9140 & $1.255 E+00$ & minute & $7.53 \mathrm{E}+01$ & $1.5953 E+09$ & $6.2683 \mathrm{E}-10$ & & $4.344 \mathrm{E}-02$ & $6.014 \mathrm{E}-02$ \\
\hline Sr 95 & 94.9194 & $2.390 \mathrm{E}+01$ & second & $2.39 \mathrm{E}+01$ & $4.9731 E+09$ & $2.0108 \mathrm{E}-10$ & & $4.614 \mathrm{E}-02$ & $5.391 \mathrm{E}-02$ \\
\hline Si 96 & 95.9217 & $1.070 E+00$ & second & $1.07 E+00$ & $1.0992 \mathrm{E}+11$ & $9.0975 E-12$ & & $3.699 \mathrm{E}-02$ & $3.897 E-02$ \\
\hline Sr 97 & 96.9261 & $4.260 \mathrm{E}-01$ & second & $4.26 \mathrm{E}-01$ & $2.7323 E+11$ & $3.6599 \mathrm{E}-12$ & & $1.971 \mathrm{E}-02$ & $2.028 \mathrm{E}-02$ \\
\hline Sr 98 & 97.9285 & $6.530 \mathrm{E}-01$ & second & $6.53 \mathrm{E}-01$ & $1.7642 \mathrm{E}+11$ & $5.6682 E-12$ & & $6.832 E-03$ & $6.853 \mathrm{E}-03$ \\
\hline Sr 99 & 98.9333 & $2.690 \mathrm{E}-01$ & second & $2.69 E-01$ & $4.2392 \mathrm{E}+11$ & $2.3589 \mathrm{E}-12$ & & $3.530 \mathrm{E}-03$ & $3.530 \mathrm{E}-03$ \\
\hline Sr 100 & 99.9354 & $2.020 \mathrm{E}-01$ & second & $2.02 \mathrm{E}-01$ & $5.5886 \mathrm{E}+11$ & $1.7893 E-12$ & & $1.519 \mathrm{E}-04$ & $1.519 E-04$ \\
\hline Sr 101 & 100.9405 & $1.180 \mathrm{E}-01$ & second & $1.18 \mathrm{E}-01$ & $9.4717 \mathrm{E}+11$ & $1.0558 \mathrm{E}-12$ & & $3.347 \mathrm{E}-05$ & $3.347 \mathrm{E}-05$ \\
\hline Si 102 & 101.9430 & $6.900 \mathrm{E}-02$ & second & $6.90 \mathrm{E}-02$ & $1.6039 \mathrm{E}+12$ & $6.2349 \mathrm{E}-13$ & & $8.708 \mathrm{E}-06$ & $8.708 \mathrm{E}-06$ \\
\hline Y 80 & 79.9343 & $3.500 \mathrm{E}+01$ & second & $3.50 \mathrm{E}+01$ & $4.0325 E+09$ & $2.4798 \mathrm{E}-10$ & & & \\
\hline $\bar{Y} 81$ & 80.9291 & $1.173 E+00$ & minute & $7.04 E+01$ & $1.9807 E+09$ & $5.0487 \mathrm{E}-10$ & & & \\
\hline Y 82 & 81.9268 & $9.500 \mathrm{E}+00$ & second & $9.50 \mathrm{E}+00$ & $1.4495 \mathrm{E}+10$ & $6.8988 \mathrm{E}-11$ & & & \\
\hline Y 83 & 82.9224 & $7.080 E+00$ & minute & $4.25 \mathrm{E}+02$ & $3.2027 \mathrm{E}+08$ & $3.1223 E-09$ & & & \\
\hline Y $83 m$ & 82.9224 & $2.850 \mathrm{E}+00$ & minute & $1.71 E+02$ & $7.9563 E+08$ & $1: 2569 \mathrm{E}-09$ & & & \\
\hline Y 84 & 83.9204 & $4.600 \mathrm{E}+00$ & second & $4.60 \mathrm{E}+00$ & $2.9225 \mathrm{E}+10$ & $3.4218 \mathrm{E}-11$ & & & \\
\hline Y 84m & 83.9204 & $3.950 \mathrm{E}+01$ & minute & $2.37 \mathrm{E}+03$ & $5.6723 E+07$ & $1.7629 \mathrm{E}-08$ & & & \\
\hline Y 85 & 84.9164 & $2.680 E+00$ & hour & $9.65 E+03$ & $1.3770 E+07$ & $7.2619 E-08$ & & & \\
\hline Y $85 m$ & 84.9164 & $4.860 E+00$ & hour & $1.75 E+04$ & $7.5936 E+06$ & $1.3169 \mathrm{E}-07$ & & & \\
\hline Y 86 & 85.9149 & $1.474 E+01$ & hour & $5.31 E+04$ & $2.4746 E+06$ & $4.0410 \mathrm{E}-07$ & & & \\
\hline Y $86 \mathrm{~m}$ & 85.9149 & $4.800 \mathrm{E}+01$ & minute & $2.88 E+03$ & $4.5595 \mathrm{E}+07$ & $2.1932 \mathrm{E}-08$ & & & \\
\hline Y 87 & 86.9109 & $3.325 E+00$ & day & $2.87 E+05$ & $4.5185 E+05$ & $2.2131 \mathrm{E}-06$ & & & \\
\hline Y $87 \mathrm{~m}$ & 86.9109 & $1.337 E+01$ & hour & $4.81 \mathrm{E}+04$ & $2.6969 \mathrm{E}+06$ & $3.7079 \mathrm{E}-07$ & & & \\
\hline Y 88 & 87.9095 & $1.067 E+02$ & day & $9.21 \mathrm{E}+06$ & $1.3927 E+04$ & $7.1802 E-05$ & & & \\
\hline Y89m & 88.9058 & $1.606 \mathrm{E}+01$ & second & $1.61 E+01$ & $7.9013 \mathrm{E}+09$ & $1.2656 \mathrm{E}-10$ & & $2.505 E-09$ & $4.885 \mathrm{E}-02$ \\
\hline$Y 90$ & 89.9072 & $2.671 E+00$ & day & $2.31 \mathrm{E}+05$ & $5.4374 E+05$ & $1.8391 \mathrm{E}-06$ & 0.005540 & 1.777E-07 & $5.905 E-02$ \\
\hline Y90m & 89.9072 & $3.190 E+00$ & hour & $1.15 \mathrm{E}+04$ & $1.0927 \mathrm{E}+07$ & $9.1519 \mathrm{E}-08$ & & $2.522 \mathrm{E}-07$ & $2.522 E-07$ \\
\hline Y91 & 90.9073 & $5.851 E+01$ & day & $5.06 \mathrm{E}+06$ & $2.4549 \mathrm{E}+04$ & $4.0735 \mathrm{E}-05$ & 0.003600 & $1.835 \mathrm{E}-06$ & $5.919 \mathrm{E}-02$ \\
\hline$\gamma 91 \mathrm{~m}$ & 90.9073 & $4.971 E+01$ & minute & $2.98 \mathrm{E}+03$ & $4.1609 E+07$ & $2.4034 \mathrm{E}-08$ & & 7.933E-06 & $3.380 \mathrm{E}-02$ \\
\hline Y92 & 91.9089 & $3.540 \mathrm{E}+00$ & hour & $1.27 E+04$ & $9.6319 \mathrm{E}+06$ & 1.0382E-07 & & $5.380 \mathrm{E}-04$ & $5.962 \mathrm{E}-02$ \\
\hline $\begin{array}{l}93 \\
\end{array}$ & 92.9096 & $1.018 \mathrm{E}+01$ & hour & $3.66 \mathrm{E}+04$ & $3.3133 E+06$ & $3.0181 \mathrm{E}-07$ & & $4.229 \mathrm{E}-06$ & $6.390 \mathrm{E}-02$ \\
\hline$\overline{Y 93 m}$ & 92.9096 & $8.200 \mathrm{E}-01$ & second & $8.20 \mathrm{E}-01$ & $1.4808 \mathrm{E}+11$ & $6.7530 \mathrm{E}-12$ & & & \\
\hline Y94 & 93.9116 & $1.870 E+01$ & minute & $1.12 E+03$ & $1.0707 E+08$ & $9.3398 \mathrm{E}-09$ & & $3.872 \mathrm{E}-03$ & $6.401 \mathrm{E}-02$ \\
\hline
\end{tabular}




\begin{tabular}{|c|c|c|c|c|c|c|c|c|c|}
\hline Isotope & $\begin{array}{l}\text { Atomic } \\
\text { Mass }\end{array}$ & Halflife & $\begin{array}{l}\text { Time } \\
\text { Units }\end{array}$ & $t_{1 / 2}, \mathbf{s e c}$ & Curies/gram & Grams/ Curle & $\begin{array}{c}\text { Watts/Ci } \\
\text { (Ref.3) }\end{array}$ & $\begin{array}{c}\text { Individual }^{9} \\
\text { Fission } \\
\text { Product Yield } \\
\text { (Ref.4) } \\
\end{array}$ & $\begin{array}{c}\text { Cumulative' } \\
\text { Fission Product } \\
\text { Yield (Ref.4) }\end{array}$ \\
\hline Y95 & 94.9128 & $1.030 \mathrm{E}+01$ & minute & $6.18 E+02$ & $1.9234 E+08$ & $5.1992 E-09$ & & $1.029 \mathrm{E}-02$ & $6.421 \mathrm{E}-02$ \\
\hline$Y 96$ & 95.9159 & $5.340 \mathrm{E}+00$ & second & $5.34 \mathrm{E}+00$ & $2.2026 E+10$ & $4.5400 \mathrm{E}-11$ & & $3.213 \mathrm{E}-03$ & $4.218 \mathrm{E}-02$ \\
\hline Y96m & 95.9159 & $9.600 \mathrm{E}+00$ & second & $9.60 \mathrm{E}+00$ & $1.2252 E+10$ & $8.1618 \mathrm{E}-11$ & & $1.790 \mathrm{E}-02$ & $1.790 \mathrm{E}-02$ \\
\hline Y97 & 96.9181 & $3.750 \mathrm{E}+00$ & second & $3.75 E+00$ & $3.1041 \mathrm{E}+10$ & $3.2215 \mathrm{E}-11$ & & $5.974 \mathrm{E}-03$ & $2.643 E-02$ \\
\hline Y97m1 & 96.9181 & $1.170 \mathrm{E}+00$ & second & $1.17 \mathrm{E}+00$ & $9.9491 \mathrm{E}+10$ & $1.0051 \mathrm{E}-11$ & & $2.583 \mathrm{E}-02$ & $2.586 E-02$ \\
\hline Y97m2 & 96.9181 & $1.420 \mathrm{E}-01$ & second & $1.42 \mathrm{E}-01$ & $8.1975 E+11$ & $1.2199 \mathrm{E}-12$ & & & \\
\hline Y98 & 97.9222 & $5.480 E-01$ & second & $5.48 \mathrm{E}-01$ & $2.1024 \mathrm{E}+11$ & $4.7565 \mathrm{E}-12$ & & $7.254 \mathrm{E}-03$ & $1.409 \mathrm{E}-02$ \\
\hline Y 98m & 97.9222 & $2.000 \mathrm{E}+00$ & second & $2.00 E+00$ & $5.7606 \mathrm{E}+10$ & $1.7359 \mathrm{E}-11$ & & $2.188 \mathrm{E}-02$ & $2.190 \mathrm{E}-02$ \\
\hline Y99 & 98.9246 & $1.470 E+00$ & second & $1.47 E+00$ & $7.7581 E+10$ & $1.2890 \mathrm{E}-11$ & & $2.018 \mathrm{E}-02$ & $2.370 E-02$ \\
\hline$Y 100$ & 99.9278 & $7.350 \mathrm{E}-01$ & second & 7.35E-01 & $1.5360 \mathrm{E}+11$ & $6.5102 \mathrm{E}-12$ & & $1.297 \mathrm{E}-03$ & $1.444 \mathrm{E}-03$ \\
\hline$Y 100 \mathrm{~m}$ & 99.9278 & $9.400 \mathrm{E}-01$ & second & $9.40 \mathrm{E}-01$ & $1.2011 \mathrm{E}+11$ & $8.3260 \mathrm{E}-12$ & & $3.914 \mathrm{E}-03$ & $3.915 \mathrm{E}-03$ \\
\hline$Y 101$ & 100.9303 & $4.480 \mathrm{E}-01$ & second & $4.48 \mathrm{E}-01$ & $2.4950 E+11$ & $4.0080 \mathrm{E}-12$ & & $4.013 E-03$ & $4.046 E-03$ \\
\hline Y102 & 101.9336 & $3.000 \mathrm{E}-01$ & second & $3.00 \mathrm{E}-01$ & $3.6892 \mathrm{E}+11$ & $2.7106 \mathrm{E}-12$ & & $2.589 \mathrm{E}-03$ & $2.597 \mathrm{E}-03$ \\
\hline$Y 102 m$ & 101.9336 & $3.600 \mathrm{E}-01$ & second & $3.60 \mathrm{E}-01$ & $3.0744 \mathrm{E}+11$ & $3.2527 \mathrm{E}-12$ & & & \\
\hline $\mathrm{Zr} 81$ & 80.9368 & $1.500 \mathrm{E}+01$ & second & $1.50 \mathrm{E}+01$ & $9.2926 \mathrm{E}+09$ & $1.0761 \mathrm{E}-10$ & & & \\
\hline $\operatorname{Zr} 82$ & 81.9311 & $3.200 \mathrm{E}+01$ & second & $3.20 E+01$ & $4.3031 E+09$ & $2.3239 \mathrm{E}-10$ & & & \\
\hline $\mathrm{Zr} 83$ & 82.9287 & $4.400 E+01$ & second & $4.40 E+01$ & $3.0919 \mathrm{E}+09$ & $3.2343 E-10$ & & & \\
\hline Zr 84 & 83.9233 & $2.590 E+01$ & minute & $1.55 \mathrm{E}+03$ & $8.6505 E+07$ & $1.1560 \mathrm{E}-08$ & & & \\
\hline $\mathrm{Zr} 85$ & 84.9215 & $7.860 \mathrm{E}+00$ & minute & $4.72 E+02$ & $2.8170 E+08$ & $3.5499 \mathrm{E}-09$ & & & \\
\hline $\mathrm{Zr} 85 \mathrm{~m}$ & 84.9215 & $1.090 E+01$ & second & $1.09 E+01$ & $1.2188 E+10$ & $8.2048 E-11$ & & & \\
\hline $\operatorname{Zr~} 86$ & 85.9165 & $1.650 \mathrm{E}+01$ & hour & $5.94 E+04$ & $2.2106 \mathrm{E}+06$ & $4.5236 \mathrm{E}-07$ & & & \\
\hline Zr 87 & 86.9148 & $1.680 \mathrm{E}+00$ & hour & $6.05 \mathrm{E}+03$ & $2.1462 E+07$ & $4.6594 \mathrm{E}-08$ & & & \\
\hline $\mathrm{Zr} 87 \mathrm{~m}$ & 86.9148 & $1.400 E+01$ & second & $1.40 \mathrm{E}+01$ & $9.2716 E+09$ & $1.0786 \mathrm{E}-10$ & & & \\
\hline $\mathrm{Zr} 88$ & 87.9102 & $8.340 E+01$ & day & $7.21 \mathrm{E}+06$ & $1.7810 E+04$ & $5.6149 \mathrm{E}-05$ & & & \\
\hline $\mathrm{Zr} 89$ & 88.9089 & $3.267 \mathrm{E}+00$ & day & $2.82 E+05$ & $4.4953 E+05$ & $2.2246 E-06$ & & $3.337 \mathrm{E}-13$ & $4.056 \mathrm{E}-13$ \\
\hline Zr 89m & 88.9089 & $4.180 \mathrm{E}+00$ & minute & $2.51 E+02$ & $5.0595 E+08$ & $1.9765 \mathrm{E}-09$ & & $7.717 E-14$ & $7.717 \mathrm{E}-14$ \\
\hline $\mathrm{Zrg0m}$ & 89.9047 & $8.092 \mathrm{E}-01$ & second & $8.09 \mathrm{E}-01$ & $1.5507 \mathrm{E}+11$ & $6.4485 \mathrm{E}-12$ & & $4.434 \mathrm{E}-11$ & $6.452 \mathrm{E}-11$ \\
\hline $\operatorname{Zr} 93$ & 92.9064 & $1.530 E+06$ & year & $4.83 E+13$ & $2.5149 \mathrm{E}-03$ & $3.9763 \mathrm{E}+02$ & 0.000290 & $4.229 \mathrm{E}-06$ & $6.390 \mathrm{E}-02$ \\
\hline Zr95 & 94.9080 & $6.402 E+01$ & day & $5.53 E+06$ & $2.1490 \mathrm{E}+04$ & $4.6532 \mathrm{E}-05$ & 0.005060 & $7.540 \mathrm{E}-04$ & $6.496 \mathrm{E}-02$ \\
\hline Zr96 & 95.9083 & $1.000 \mathrm{E}+18$ & year & $3.16 \mathrm{E}+25$ & $3.7274 \mathrm{E}-15$ & $2.6828 \mathrm{E}+14$ & & $2.518 E-03$ & $6.264 \mathrm{E}-02$ \\
\hline Zr97 & 96.9110 & $1.691 \mathrm{E}+01$ & hour & $6.09 E+04$ & $1.9123 E+06$ & $5.2293 \mathrm{E}-07$ & & $7.181 \mathrm{E}-03$ & $5.995 \mathrm{E}-02$ \\
\hline $\operatorname{Zr98}$ & 97.9127 & $3.070 \mathrm{E}+01$ & second & $3.07 E+01$ & $3.7532 \mathrm{E}+09$ & $2.6644 \mathrm{E}-10$ & & $3.268 \mathrm{E}-02$ & $5.619 \mathrm{E}-02$ \\
\hline $\mathrm{Zr99}$ & 98.9165 & $2.100 \mathrm{E}+00$ & second & $2.10 \mathrm{E}+00$ & $5.4311 E+10$ & $1.8412 \mathrm{E}-11$ & & $3.268 E-02$ & $5.619 E-02$ \\
\hline $\operatorname{Zr} 100$ & 99.9178 & $7.100 E+00$ & second & $7.10 E+00$ & $1.5903 E+10$ & $6.2882 \mathrm{E}-11$ & & $5.245 \mathrm{E}-02$ & $5.778 \mathrm{E}-02$ \\
\hline $\mathrm{Zr} 101$ & 100.9211 & $2.100 \mathrm{E}+00$ & second & $2.10 E+00$ & $5.3232 E+10$ & $1.8786 \mathrm{E}-11$ & & $2.642 E-02$ & $3.055 E-02$ \\
\hline
\end{tabular}

WSRC-TR-99-00344

October, 1999

page 18 


\begin{tabular}{|c|c|c|c|c|c|c|c|c|c|}
\hline Isotope & $\begin{array}{l}\text { Atomic } \\
\text { Mass }\end{array}$ & Halflife & $\begin{array}{l}\text { Time } \\
\text { Units }\end{array}$ & $t_{1 / 2}, \sec$ & Curies/ gram & Grams/ Curie & $\begin{array}{c}\text { Watts/Ci } \\
\text { (Ref.3) }\end{array}$ & $\begin{array}{c}\text { Individual }^{9} \\
\text { Fission } \\
\text { Product Yield } \\
\text { (Ref.4) }\end{array}$ & $\begin{array}{c}\text { Cumulative } \\
\text { Fission Product } \\
\text { Yield (Ref.4) }\end{array}$ \\
\hline Zr 102 & 101.9230 & $2.900 E+00$ & second & $2.90 E+00$ & $3.8169 \mathrm{E}+10$ & $2.6200 \mathrm{E}-11$ & & $2.081 \mathrm{E}-02$ & $2.341 \mathrm{E}-02$ \\
\hline $2 \times 103$ & 102.9266 & $1.300 E+00$ & second & $1.30 E+00$ & $8.4315 E+10$ & $1.1860 \mathrm{E}-11$ & & $5.413 \mathrm{E}-03$ & $6.061 \mathrm{E}-03$ \\
\hline Zr 104 & 103.9288 & $1.200 \mathrm{E}+00$ & second & $1.20 \mathrm{E}+00$ & $9.0461 E+10$ & $1.1055 \mathrm{E}-11$ & & $1.129 \mathrm{E}-03$ & $1.132 \mathrm{E}-03$ \\
\hline $\mathrm{Nb} 86$ & 85.9250 & $1.467 E+00$ & minute & $8.80 E+01$ & $1.4920 \mathrm{E}+09$ & $6.7025 E-10$ & & & \\
\hline Nb 87 & 86.9204 & $2.600 E+00$ & minute & $1.56 \mathrm{E}+02$ & $8.3201 E+08$ & $1.2019 \mathrm{E}-09$ & & & \\
\hline $\mathrm{Nb} 87 \mathrm{~m}$ & 86.9204 & $3.700 \mathrm{E}+00$ & minute & $2.22 E+02$ & $5.8466 \mathrm{E}+08$ & $1.7104 \mathrm{E}-09$ & & & \\
\hline $\mathrm{Nb} 88$ & 87.9180 & $1.450 \mathrm{E}+01$ & minute & $8.70 E+02$ & $1.4750 E+08$ & $6.7799 \mathrm{E}-09$ & & & \\
\hline Nb 88m & 87.9180 & $7.800 E+00$ & minute & $4.68 E+02$ & $2.7419 E+08$ & $3.6471 \mathrm{E}-09$ & & & \\
\hline $\mathrm{Nb} 89$ & 88.9135 & $1.900 E+00$ & hour & $6.84 E+03$ & $1.8550 \mathrm{E}+07$ & $5.3907 \mathrm{E}-08$ & & & \\
\hline $\mathrm{Nb} 89 \mathrm{~m}$ & 88.9135 & $1.180 E+00$ & hour & $4.25 \mathrm{E}+03$ & $2.9869 \mathrm{E}+07$ & $3.3479 \mathrm{E}-08$ & & & \\
\hline $\mathrm{Nb} 90$ & 89.9113 & $1.460 \mathrm{E}+01$ & hour & $5.26 E+04$ & $2.3873 E+06$ & $4.1888 \mathrm{E}-07$ & & & \\
\hline $\mathrm{Nb} 90 \mathrm{~m}$ & 89.9113 & $1.881 \mathrm{E}+01$ & second & $1.88 \mathrm{E}+01$ & $6.6707 \mathrm{E}+09$ & $1.4991 \mathrm{E}-10$ & & & \\
\hline$\overline{\mathrm{Nb} 91}$ & 90.9070 & $6.800 E+02$ & year & $2.15 E+10$ & $5.7830 E+00$ & $1.7292 \mathrm{E}-01$ & & & \\
\hline $\mathrm{Nb} 91 \mathrm{~m}$ & 90.9070 & $6.086 \mathrm{E}+01$ & day & $5.26 E+06$ & $2.3601 \mathrm{E}+04$ & $4.2371 \mathrm{E}-05$ & & & \\
\hline $\mathrm{Nb} 92$ & 91.9072 & $3.470 \mathrm{E}+07$ & year & $1.10 \mathrm{E}+15$ & $1.1209 \mathrm{E}-04$ & $8.9211 E+03$ & & & \\
\hline $\mathrm{Nb} 92 \mathrm{~m}$ & 91.9072 & $1.015 E+01$ & day & $8.77 \mathrm{E}+05$ & $1.3997 E+05$ & $7.1442 \mathrm{E}-06$ & & $1.517 \mathrm{E}-12$ & $1.517 \mathrm{E}-12$ \\
\hline $\mathrm{Nb} 93 \mathrm{~m}$ & 92.9064 & $1.613 E+01$ & year & $5.09 E+08$ & $2.3855 E+02$ & $4.1920 \mathrm{E}-03$ & 0.000177 & $7.531 \mathrm{E}-11$ & $6.071 \mathrm{E}-02$ \\
\hline Nb 94 & 93.9073 & $2.030 E+04$ & year & $6.41 E+11$ & $1.8753 \mathrm{E}-01$ & $5.3325 E+00$ & 0.010180 & $9.900 \mathrm{E}-09$ & $1.684 \mathrm{E}-08$ \\
\hline $\mathrm{Nb} 94 \mathrm{~m}$ & 93.9073 & $6.263 E+00$ & minute & $3.76 E+02$ & $3.1970 E+08$ & $3.1279 \mathrm{E}-09$ & & $6.976 E-09$ & $6.976 E-09$ \\
\hline Nb 95 & 94.9068 & $3: 498 \mathrm{E}+01$ & day & $3.02 E+06$ & $3.9338 \mathrm{E}+04$ & $2.5421 \mathrm{E}-05$ & 0.004788 & $1.032 E-06$ & $6.495 \mathrm{E}-02$ \\
\hline Nb 95m & 94.9068 & $8.660 E+01$ & hour & $3.12 \mathrm{E}+05$ & $3.8129 \mathrm{E}+05$ & $2.6227 \mathrm{E}-06$ & & $2.387 \mathrm{E}-07$ & $5.849 \mathrm{E}-04$ \\
\hline Nb 96 & 95.9081 & $2.335 E+01$ & hour & $8.41 E+04$ & $1.3994 E+06$ & $7.1461 \mathrm{E}-07$ & & $5.589 \mathrm{E}-06$ & $5.589 \mathrm{E}-06$ \\
\hline $\mathrm{Nb} 97$ & 96.9081 & $1.202 \mathrm{E}+00$ & hour, & $4.33 \mathrm{E}+03$ & $2.6910 \mathrm{E}+07$ & $3.7161 \mathrm{E}-08$ & & $1.035 \mathrm{E}-04$ & $6.008 \mathrm{E}-02$ \\
\hline $\mathrm{Nb} 97 \mathrm{~m}$ & 96.9081 & $5.270 \mathrm{E}+01$ & second & $5.27 \mathrm{E}+01$ & $2.2091 \mathrm{E}+09$ & $4.5268 \mathrm{E}-10$ & & $2.394 \mathrm{E}-05$ & $5.836 \mathrm{E}-02$ \\
\hline Nb 98 & 97.9103 & $2.860 E+00$ & second & $2.86 E+00$ & $4.0289 E+10$ & $2.4821 \mathrm{E}-11$ & & $1.049 \mathrm{E}-04$ & $5.676 \mathrm{E}-02$ \\
\hline Nb 98m & 97.9103 & $5.130 E+01$ & minute & $3.08 E+03$ & $3.7435 E+07$ & $2.6713 E-08$ & & $3.165 E-04$ & $3.165 E-04$ \\
\hline Nb 99 & 98.9116 & $1.500 \mathrm{E}+01$ & second & $1.50 \mathrm{E}+01$ & $7.6039 \mathrm{E}+09$ & $1.3151 \mathrm{E}-10$ & & $3.357 \mathrm{E}-03$ & $3.932 \mathrm{E}-02$ \\
\hline $\mathrm{Nb} 99 \mathrm{~m}$ & 98.9116 & $2.600 \mathrm{E}+00$ & minute & $1.56 E+02$ & $7.3115 \mathrm{E}+08$ & $1.3677 \mathrm{E}-09$ & & $7.762 \mathrm{E}-04$ & $2.101 \mathrm{E}-02$ \\
\hline Nb 100 & 99.9142 & $1.500 \mathrm{E}+00$ & second & $1.50 E+00$ & $7.5276 \mathrm{E}+10$ & $1.3284 \mathrm{E}-11$ & & $9.863 \mathrm{E}-04$ & $5.761 \mathrm{E}-02$ \\
\hline $\mathrm{Nb} 100 \mathrm{~m}$ & 99.9142 & $2.990 E+00$ & second & $2.99 E+00$ & $3.7764 E+10$ & $2.6480 \mathrm{E}-11$ & & $2.976 \mathrm{E}-03$ & 4.131E-03 \\
\hline $\mathrm{Nb} 101$ & 100.9153 & $7.100 E+00$ & second & $7.10 \mathrm{E}+00$ & $1.5746 \mathrm{E}+10$ & $6.3509 \mathrm{E}-11$ & & $1.878 \mathrm{E}-02$ & $4.933 \mathrm{E}-02$ \\
\hline $\mathrm{Nb} 102$ & 101.9180 & $1.300 E+00$ & second & $1.30 \mathrm{E}+00$ & $8.5149 \mathrm{E}+10$ & $1.1744 \mathrm{E}-11$ & & $3.905 \mathrm{E}-03$ & $2.731 \mathrm{E}-02$ \\
\hline $\mathrm{Nb} 102 \mathrm{~m}$ & 101.9180 & $4.300 \mathrm{E}+00$ & second & $4.30 \mathrm{E}+00$ & $2.5743 \mathrm{E}+10$ & $3.8846 \mathrm{E}-11$ & & $1.178 \mathrm{E}-02$ & $1.178 \mathrm{E}-02$ \\
\hline $\mathrm{Nb} 103$ & 102.9191 & $1.500 E+00$ & second & $1.50 E+00$ & $7.3078 E+10$ & $1.3684 \mathrm{E}-11$ & & $1.621 \mathrm{E}-02$ & $2.227 \mathrm{E}-02$ \\
\hline $\mathrm{Nb} 104$ & 103.9225 & $4.800 \mathrm{E}+00$ & second & $4.80 E+00$ & $2.2617 \mathrm{E}+10$ & $4.4215 \mathrm{E}-11$ & & $4.423 \mathrm{E}-03$ & $5.557 \mathrm{E}-03$ \\
\hline
\end{tabular}




\begin{tabular}{|c|c|c|c|c|c|c|c|c|c|}
\hline Isotope & $\begin{array}{l}\text { Atomic } \\
\text { Mass }\end{array}$ & Halflife & $\begin{array}{l}\text { Time } \\
\text { Units }\end{array}$ & $t_{1 / 2}, \sec$ & Curies/ gram & Grams/ Curie & $\begin{array}{c}\text { Watts/CI } \\
\text { (Ref.3) }\end{array}$ & $\begin{array}{c}\text { Individual }^{1} \\
\text { Fission } \\
\text { Product Yield } \\
\text { (Ref.4) }\end{array}$ & $\begin{array}{c}\text { Cumulative } \\
\text { Fission Product } \\
\text { Yield (Ref.4) }\end{array}$ \\
\hline $\mathrm{Nb} 104 \mathrm{~m}$ & 103.9225 & $9.200 \mathrm{E}-01$ & second & $9.20 \mathrm{E}-01$ & $1.1800 \mathrm{E}+11$ & $8.4746 \mathrm{E}-12$ & & $1.889 \mathrm{E}-03$ & $1.889 \mathrm{E}-03$ \\
\hline $\mathrm{Nb} 105$ & 104.9239 & $2.950 E+00$ & second & $2.95 \mathrm{E}+00$ & $3.6449 \mathrm{E}+10$ & $2.7436 \mathrm{E}-11$ & & $1.701 \mathrm{E}-03$ & $2.587 \mathrm{E}-03$ \\
\hline $\mathrm{Nb} 106$ & 105.9282 & $1.020 \mathrm{E}+00$ & second & $1.02 E+00$ & $1.0442 E+11$ & $9.5771 \mathrm{E}-12$ & & $1.453 \mathrm{E}-04$ & $1.472 E-04$ \\
\hline $\mathrm{Nb} 107$ & 106.9303 & $3.300 \mathrm{E}-01$ & second & $3.30 \mathrm{E}-01$ & $3.1971 \mathrm{E}+11$ & $3.1278 \mathrm{E}-12$ & & $2.051 \mathrm{E}-05$ & $2.053 E-05$ \\
\hline Mo 88 & 87.9220 & $8.000 E+00$ & minute & $4.80 E+02$ & $2.6732 E+08$ & $3.7408 \mathrm{E}-09$ & & & \\
\hline Mo 89 & 88.9195 & $2.040 E+00$ & minute & $1.22 E+02$ & $1.0366 E+09$ & $9.6472 \mathrm{E}-10$ & & & \\
\hline Mo $89 \mathrm{~m}$ & 88.9195 & $1.900 \mathrm{E}-01$ & second & $1.90 \mathrm{E}-01$ & $6.6777 E+11$ & $1.4975 \mathrm{E}-12$ & & & \\
\hline Mo 90 & 89.9139 & $5.670 E+00$ & hour & $2.04 E+04$ & $6.1470 E+06$ & $1.6268 \mathrm{E}-07$ & & & \\
\hline Mo91 & 90.9118 & $1.549 E+01$ & minute & $9.29 \mathrm{E}+02$ & $1.3352 E+08$ & $7.4894 \mathrm{E}-09$ & & & \\
\hline Mo91m & 90.9118 & $1.083 E+00$ & minute & $6.50 E+01$ & $1: 9098 E+09$ & $5.2363 \mathrm{E}-10$ & & & \\
\hline Mo 93 & 92.9068 & $4.000 E+03$ & year & $1.26 E+11$ & $9.6195 \mathrm{E}-01$ & $1.0396 \mathrm{E}+00$ & 0.000094 & $3.473 E-16$ & $5.593 \mathrm{E}-16$ \\
\hline Mo 93m & 92.9068 & $6.850 \mathrm{E}+00$ & hour & $2.47 E+04$ & $4.9242 \mathrm{E}+06$ & $2.0308 \mathrm{E}-07$ & & $2.122 \mathrm{E}-16$ & $2.122 E-16$ \\
\hline Mo 99 & 98.9077 & $2.748 \mathrm{E}+00$ & day & $2.37 E+05$ & $4.8050 \mathrm{E}+05$ & $2.0812 E-06$ & 0.004028 & $7.778 E-04$ & $6.110 \mathrm{E}-02$ \\
\hline Mo 101 & 100.9103 & $1.461 \mathrm{E}+01$ & minute & $8.77 E+02$ & $1.2754 E+08$ & $7.8408 \mathrm{E}-09$ & & $1.483 \mathrm{E}-03$ & $5.081 \mathrm{E}-02$ \\
\hline Mo 102 & 101.9103 & $1.130 E+01$ & minute & $6.78 \mathrm{E}+02$ & $1.6328 \mathrm{E}+08$ & $6.1245 \mathrm{E}-09$ & & $3.198 \mathrm{E}-03$ & $4.229 \mathrm{E}-02$ \\
\hline Mo 103 & 102.9132 & $1.125 E+00$ & minute & $6.75 E+01$ & $1.6241 E+09$ & $6.1574 \mathrm{E}-10$ & & $7.227 E-03$ & $2.953 E-02$ \\
\hline Mo 104 & 103.9138 & $1.000 \mathrm{E}+00$ & minute & $6.00 \mathrm{E}+01$ & $1.8095 E+09$ & $5.5265 \mathrm{E}-10$ & & $1.032 \mathrm{E}-02$ & $1.785 \mathrm{E}-02$ \\
\hline Mo 105 & 104.9170 & $3.560 \mathrm{E}+01$ & second & $3.56 \mathrm{E}+01$ & $3.0205 E+09$ & $3.3107 \mathrm{E}-10$ & & $6.865 \mathrm{E}-03$ & $9.340 \mathrm{E}-03$ \\
\hline Mo 106 & 105.9181 & $8.400 E+00$ & second & $8.40 E+00$ & $1.2680 \mathrm{E}+10$ & $7.8863 \mathrm{E}-11$ & & $3.555 E-03$ & $3.700 \mathrm{E}-03$ \\
\hline Mo 107 & 106.9217 & $3.500 \mathrm{E}+00$ & second & $3.50 E+00$ & $3.0147 E+10$ & $3.3171 \mathrm{E}-11$ & & $1.012 \mathrm{E}-03$ & $1.030 \mathrm{E}-03$ \\
\hline Mo 108 & 107.9236 & $1.500 E+00$ & second & $1.50 E+00$ & $6.9690 \mathrm{E}+10$ & $1.4349 \mathrm{E}-11$ & & $5.076 \mathrm{E}-05$ & $5.096 \mathrm{E}-05$ \\
\hline Mo 109 & 108.9278 & $5.300 \mathrm{E}-01$ & second & $5.30 \mathrm{E}-01$ & $1.9542 E+11$ & $5.1173 \mathrm{E}-12$ & & $3.809 \mathrm{E}-06$ & $3.814 E-06$ \\
\hline TC 89 & 88.9275 & $1.280 E+01$ & second & $1.28 E+01$ & $9.9113 E+09$ & $1.0090 \mathrm{E}-10$ & & & \\
\hline Tc $89 \mathrm{~m}$ & 88.9275 & $1.290 E+01$ & second & $1.29 \mathrm{E}+01$ & $9.8345 E+09$ & $1.0168 \mathrm{E}-10$ & & & \\
\hline To 90 & 89.9236 & $8.700 E+00$ & second & $8.70 E+00$ & $1.4421 \mathrm{E}+10$ & $6.9345 \mathrm{E}-11$ & & & \\
\hline Tc $90 \mathrm{~m}$ & 89.9236 & $4.920 \mathrm{E}+01$ & second & $4.92 \mathrm{E}+01$ & $2.5500 \mathrm{E}+09$ & $3.9216 \mathrm{E}-10$ & & & \\
\hline TC 91 & 90.9184 & $3.140 E+00$ & minute & $1.88 E+02$ & $6.5863 E+08$ & $1.5183 \mathrm{E}-09$ & & & \\
\hline Tc 91m & 90.9184 & $3.300 E+00$ & minute & $1.98 \mathrm{E}+02$ & $6.2670 \mathrm{E}+08$ & $1.5957 \mathrm{E}-09$ & & & \\
\hline TC 92 & 91.9153 & $4.230 \mathrm{E}+00$ & minute & $2.54 \mathrm{E}+02$ & $4.8361 E+08$ & $2.0678 \mathrm{E}-09$ & & & \\
\hline Tc 93 & 92.9102 & $2.750 E+00$ & hour & $9.90 E+03$ & $1.2265 \mathrm{E}+07$ & $8.1531 E-08$ & & & \\
\hline Tc 93m & 92.9102 & $4.350 \mathrm{E}+01$ & minute & $2.61 E+03$ & $4.6523 \mathrm{E}+07$ & $2.1495 \mathrm{E}-08$ & & & \\
\hline TC 94 & 93.9097 & $4.883 E+00$ & hour & $1.76 \mathrm{E}+04$ & $6.8336 \mathrm{E}+06$ & $1.4634 \mathrm{E}-07$ & & & \\
\hline Tc $94 \mathrm{~m}$ & 93.9097 & $5.200 \mathrm{E}+01$ & minute & $3.12 \mathrm{E}+03$ & $3.8505 \mathrm{E}+07$ & $2.5971 \mathrm{E}-08$ & & & \\
\hline TC95 & 94.9077 & $2.000 E+01$ & hour & $7.20 E+04$ & $1.6510 \mathrm{E}+06$ & $6.0570 \mathrm{E}-07$ & & & \\
\hline Te $95 \mathrm{~m}$ & 94.9077 & $6.100 \mathrm{E}+01$ & day & $5.27 E+06$ & $2.2554 E+04$ & $4.4337 \mathrm{E}-05$ & & & \\
\hline
\end{tabular}




\begin{tabular}{|c|c|c|c|c|c|c|c|c|c|}
\hline Isotope & $\begin{array}{l}\text { Atomic } \\
\text { Mass }\end{array}$ & Halflife & $\begin{array}{l}\text { Time } \\
\text { Units }\end{array}$ & $t_{1 / 2}, \sec$ & Curies/gram & Grams/Curie & $\begin{array}{c}\text { Watts/Cl } \\
\text { (Ref.3) }\end{array}$ & $\begin{array}{c}\text { Individual }^{1} \\
\text { Fission } \\
\text { Product Yield } \\
\text { (Ref.4) }^{\text {Ref }} \\
\end{array}$ & $\begin{array}{l}\text { Cumulative }^{i} \\
\text { Fission Product } \\
\text { Yield (Ref.4) }\end{array}$ \\
\hline Tc 96 & 95.9079 & $4.280 \mathrm{E}+00$ & day & $3.70 E+05$ & $3.1810 E+05$ & $3.1437 \mathrm{E}-06$ & & & \\
\hline Tc 96m & 95.9079 & $5.150 E+01$ & minute & $3.09 E+03$ & $3.8068 E+07$ & $2.6269 \mathrm{E}-08$ & & & \\
\hline TC 97 & 96.9064 & $2.600 E+06$ & year & $8.21 E+13$ & $1.4188 \mathrm{E}-03$ & $7.0480 E+02$ & & & \\
\hline Tc $97 \mathrm{~m}$. & 96.9064 & $9.010 E+01$ & day & $7.78 \mathrm{E}+06$ & $1.4955 E+04$ & $6.6867 \mathrm{E}-05$ & & $8.581 \mathrm{E}-13$ & $8.581 E-13$ \\
\hline Tc 98 & 97.9072 & $4.200 \mathrm{E}+06$ & year & $1.33 E+14$ & $8.6935 E-04$ & $1.1503 E+03$ & & $7.228 \mathrm{E}-09$ & 7.228E-09 \\
\hline TC 99 & 98.9063 & $2.111 E+05$ & year & $6.66 E+12$ & $1.7122 \mathrm{E}-02$ & $5.8405 E+01$ & 0.000501 & 1.173E-08 & $6.110 \mathrm{E}-02$ \\
\hline To 99m & 98.9063 & $6.010 \mathrm{E}+00$ & hour & $2.16 E+04$ & $5.2720 E+06$ & $1.8968 \mathrm{E}-07$ & 0.000819 & $2.713 E-09$ & $5.316 \mathrm{E}-02$ \\
\hline TC 100 & 99.9077 & $1.580 \mathrm{E}+01$ & second & $1.58 \mathrm{E}+01$ & $7.1469 E+09$ & $1.3992 \mathrm{E}-10$ & & $3.029 \mathrm{E}-07$ & $3.029 \mathrm{E}-07$ \\
\hline TC 101 & 100.9073 & $1.422 E+01$ & minute & $8.53 E+02$ & $1.3104 E+08$ & 7.6313E-09 & & $4.579 \mathrm{E}-06$ & $5.081 \mathrm{E}-02$ \\
\hline TC 102 & 101.9092 & $5.280 \mathrm{E}+00$ & second & $5.28 \mathrm{E}+00$ & $2.0967 E+10$ & $4.7695 \mathrm{E}-11$ & & $9.926 \mathrm{E}-06$ & $4.230 \mathrm{E}-02$ \\
\hline Tc $102 \mathrm{~m}$ & 101.9092 & $4.350 E+00$ & minute & $2.61 E+02$ & $4.2415 E+08$ & $2.3576 E-09$ & & $2.995 E-05$ & $2.995 \mathrm{E}-05$ \\
\hline Tc 103 & 102.9092 & $5.420 \mathrm{E}+01$ & second & $5.42 \mathrm{E}+01$ & $2.0227 E+09$ & $4.9440 \mathrm{E}-10$ & & 7.411E-04 & $3.027 \mathrm{E}-02$ \\
\hline Tc 104 & 103.9114 & $1.830 E+01$ & minute & $1.10 E+03$ & $9.8881 \mathrm{E}+07$ & $1.0113 \mathrm{E}-08$ & & $5.463 \mathrm{E}-04$ & $1.839 \mathrm{E}-02$ \\
\hline Tc 105 & 104.9117 & $7.600 \mathrm{E}+00$ & minute & $4.56 \mathrm{E}+02$ & $2.3582 E+08$ & $4.2405 \mathrm{E}-09$ & & $2.720 \mathrm{E}-04$ & $9.612 \mathrm{E}-03$ \\
\hline TC 106 & 105.9144 & $3.560 E+01$ & second & $3.56 \mathrm{E}+01$ & $2.9921 \mathrm{E}+09$ & $3.3422 \mathrm{E}-10$ & & $2.278 \mathrm{E}-04$ & $3.928 \mathrm{E}-03$ \\
\hline Tc 107 & 106.9151 & $2.120 \mathrm{E}+01$ & second & $2.12 E+01$ & $4.9774 E+09$ & $2.0091 \mathrm{E}-10$ & & $1.750 E-04$ & $1.205 \mathrm{E}-03$ \\
\hline TC 108 & 107.9185 & $5.170 E+00$ & second & $5.17 \mathrm{E}+00$ & $2.0220 \mathrm{E}+10$ & $4.9455 \mathrm{E}-11$ & & $2.645 E-04$ & $3.154 \mathrm{E}-04$ \\
\hline TC 109 & 108.9196 & $8.700 \mathrm{E}-01$ & second & $8.70 E-01$ & $1.1906 \mathrm{E}+11$ & $8.3994 \mathrm{E}-12$ & & $7.604 \mathrm{E}-05$ & $7.985 \mathrm{E}-05$ \\
\hline TC 110 & 109.9234 & $9.200 \mathrm{E}-01$ & second & $9.20 \mathrm{E}-01$ & $1.1156 E+11$ & $8.9640 \mathrm{E}-12$ & & $2.604 \mathrm{E}-05$ & $2.667 E-05$ \\
\hline TC 111 & 110.9251 & $3.000 \mathrm{E}-01$ & second & $3.00 \mathrm{E}-01$ & $3.3902 E+11$ & $2.9497 \mathrm{E}-12$ & & $7.028 \mathrm{E}-06$ & 7.084E-06 \\
\hline TC 113 & 112.9313 & $1.300 \mathrm{E}-01$ & second & $1.30 \mathrm{E}-01$ & $7.6846 \mathrm{E}+11$ & $1.3013 \mathrm{E}-12$ & & $3.569 \mathrm{E}-07$ & $3.569 \mathrm{E}-07$ \\
\hline Ru 90 & 89.9298 & $1.300 E+01$ & second & $1.30 E+01$ & $9.6501 \mathrm{E}+09$ & $1.0363 \mathrm{E}-10$ & & & \\
\hline Ru 91 & 90.9264 & $9.000 \mathrm{E}+00$ & second & $9.00 \mathrm{E}+00$ & $1.3786 E+10$ & $7.2536 \mathrm{E}-11$ & & & \\
\hline Ru 91m & 90.9264 & $7.600 \mathrm{E}+00$ & second & $7.60 \mathrm{E}+00$ & $1.6326 \mathrm{E}+10$ & $6.1253 \mathrm{E}-11$ & & & \\
\hline Ru92 & 91.9201 & $3.650 \mathrm{E}+00$ & minute & $2.19 E+02$ & $5.6043 E+08$ & $1.7843 \mathrm{E}-09$ & & & \\
\hline Ru93 & 92.9171 & $5.970 E+01$ & second & $5.97 \mathrm{E}+01$ & $2.0338 \mathrm{E}+09$ & 4.9169E-10 & & & \\
\hline Ru 93m & 92.9171 & $1.080 E+01$ & second & $1.08 E+01$ & $1.1242 E+10$ & $8.8949 \mathrm{E}-11$ & & & \\
\hline Ru 94 & 93.9114 & $5.180 E+01$ & minute & $3.11 \mathrm{E}+03$ & $3.8652 \mathrm{E}+07$ & $2.5872 E-08$ & & & \\
\hline Ru 95 & 94.9104 & $1.643 E+00$ & hour & $5.91 E+03$ & $2.0097 \mathrm{E}+07$ & 4.9760 E- 08 & & & \\
\hline Ru 97 & 96.9076 & $2.900 E+00$ & day & $2.51 E+05$ & $4.6463 \mathrm{E}+05$ & $2.1523 \mathrm{E}-06$ & & & \\
\hline Ru 103 & 102.9063 & $3.926 E+01$ & day & $3.39 E+06$ & $3.2320 \mathrm{E}+04$ & 3.0941E-05 & 0.003530 & $5.799 \mathrm{E}-07$ & $3.027 \mathrm{E}-02$ \\
\hline $\mathrm{Ru} 103 \mathrm{~m}$ & 102.9063 & $1.690 \mathrm{E}-03$ & second & $1.69 \mathrm{E}-03$ & $6.4871 \mathrm{E}+13$ & 1.5415E-14 & & & \\
\hline Ru 105 & 104.9078 & $4.440 \mathrm{E}+00$ & hour & $1.60 E+04$ & $6.7280 \mathrm{E}+06$ & $1.4863 E-07$ & & $2.683 E-05$ & $9.638 \mathrm{E}-03$ \\
\hline Ru 106 & 105.9073 & $3.736 E+02$ & day & $3.23 E+07$ & $3.3002 E+03$ & $3.0301 E-04$ & 0.000595 & $9.553 E-05$ & $4.023 E-03$ \\
\hline Ru 107 & 106.9099 & $3.750 \mathrm{E}+00$ & minute & $2.25 \mathrm{E}+02$ & $4.6900 \mathrm{E}+08$ & 2.1322 E-09 & & $1.960 \mathrm{E}-04$ & $1.401 \mathrm{E}-03$ \\
\hline
\end{tabular}




\begin{tabular}{|c|c|c|c|c|c|c|c|c|c|}
\hline Isotope & $\begin{array}{c}\text { Atomic } \\
\text { Mass }\end{array}$ & Halflife & $\begin{array}{l}\text { Time } \\
\text { Units }\end{array}$ & $t_{12}, \sec$ & Curies/ gram & Grams/ Curie & $\begin{array}{c}\text { Watts/Ci } \\
\text { (Ref.3) }\end{array}$ & $\begin{array}{c}\text { Individual }^{1} \\
\text { Fission }^{-} \\
\text {Product Yield } \\
\text { (Ref,4) }\end{array}$ & $\begin{array}{l}\text { Cumulative' } \\
\text { Fission Product } \\
\text { Yield (Ref.4) }\end{array}$ \\
\hline Ru 108 & 107.9102 & $4.550 E+00$ & minute & $2.73 E+02$ & $3.8296 \mathrm{E}+08$ & $2.6113 E-09$ & & $3.425 \mathrm{E}-04$ & $6.581 E-04$ \\
\hline Ru 109 & 108.9132 & $3.450 E+01$ & second & $3.45 E+01$ & $3.0025 \mathrm{E}+09$ & $3.3306 \mathrm{E}-10$ & & $6.899 \mathrm{E}-05$ & $1.487 \mathrm{E}-04$ \\
\hline Ru 110 & 109.9140 & $1.460 E+01$ & second & $1.46 \mathrm{E}+01$ & $7.0302 E+09$ & $1.4224 \mathrm{E}-10$ & & $1.666 \mathrm{E}-04$ & $1.667 \mathrm{E}-04$ \\
\hline Ru 111 & 110.9176 & $2.120 E+00$ & second & $2.12 E+00$ & $4.7978 \mathrm{E}+10$ & $2.0843 E-11$ & & 1.147E-04 & $1.215 \mathrm{E}-04$ \\
\hline Ru 112 & 111.9186 & $1.750 E+00$ & second & $1.75 \mathrm{E}+00$ & $5.7602 \mathrm{E}+10$ & $1.7361 \mathrm{E}-11$ & & 6.687E-05 & $6.839 \mathrm{E}-05$ \\
\hline Ru 113 & 112.9225 & $8.000 \mathrm{E}-01$ & second & $8.00 E-01$ & $1.2488 E+11$ & $8.0074 \mathrm{E}-12$ & & $3.102 \mathrm{E}-05$ & $3.138 \mathrm{E}-05$ \\
\hline Ru 114 & 113.9240 & $5.000 \mathrm{E}-01$ & second & $5.00 E-01$ & $1.9806 \mathrm{E}+11$ & $5.0490 \mathrm{E}-12$ & & $1.105 \mathrm{E}-05$ & 1.109E-05 \\
\hline Ru 115 & 114.9283 & $4.000 E-01$ & second & $4.00 \mathrm{E}-01$ & $2.4541 E+11$ & $4.0748 \mathrm{E}-12$ & & 1.879E-06 & $1.880 \mathrm{E}-06$ \\
\hline $\begin{array}{l}\operatorname{Rh} 94 \\
\end{array}$ & 93.9217 & $2.580 E+01$ & second & $2.58 E+01$ & $4.6558 \mathrm{E}+09$ & $2.1479 \mathrm{E}-10$ & & & \\
\hline $\operatorname{Rh~} 94 \mathrm{~m}$ & 93.9217 & $7.060 E+01$ & second & $7.06 \mathrm{E}+01$ & $1.7014 E+09$ & $5.8775 \mathrm{E}-10$ & & & \\
\hline Rh 95 & 94.9159 & $5.020 E+00$ & minute & $3.01 E+02$ & $3.9462 E+08$ & $2.5341 E-09$ & & & \\
\hline Rh 95m & 94.9159 & $1.960 \mathrm{E}+00$ & minute & $1.18 \mathrm{E}+02$ & $1.0107 \mathrm{E}+09$ & $9.8940 \mathrm{E}-10$ & & & \\
\hline$\overline{R h ~ 96}$ & 95.9145 & $9.900 E+00$ & minute & $5.94 \mathrm{E}+02$ & $1.9802 E+08$ & $5.0500 \mathrm{E}-09$ & & & \\
\hline Rh 96m & 95.9145 & $1.510 E+00$ & minute & $9.06 E+01$ & $1.2983 E+09$ & $7.7026 \mathrm{E}-10$ & & & \\
\hline Rh 97 & 96.9113 & $3.070 E+01$ & minute & $1.84 E+03$ & $6.3199 \mathrm{E}+07$ & $1.5823 \mathrm{E}-08$ & & & \\
\hline $\mathrm{Rh} 97 \mathrm{~m}$ & 96.9113 & $4.620 \mathrm{E}+01$ & minute & $2.77 E+03$ & $4.1996 \mathrm{E}+07$ & $2.3812 \mathrm{E}-08$ & & & \\
\hline $\operatorname{Rh} 98$ & 97.9107 & $8.700 E+00$ & minute & $5.22 E+02$ & $2.2074 E+08$ & $4.5303 \mathrm{E}-09$ & & & \\
\hline Rh 98m & 97.9107 & $3.500 E+00$ & minute & $2.10 \mathrm{E}+02$ & $5.4869 \mathrm{E}+08$ & $1.8225 \mathrm{E}-09$ & & & \\
\hline Rh 99 & 98.9081 & $1.610 E+01$ & day & $1.39 E+06$ & $8.1998 E+04$ & $1.2195 \mathrm{E}-05$ & & & \\
\hline Rh 99m & 98.9081 & $4.700 E+00$ & hour & $1.69 E+04$ & $6.7413 E+06$ & $1.4834 \mathrm{E}-07$ & & & \\
\hline Rh 100 & 99.9081 & $2.080 E+01$ & hour & $7.49 E+04$ & $1.5080 \mathrm{E}+06$ & $6.6312 \mathrm{E}-07$ & & & \\
\hline Rh 101 & 100.9062 & $3.300 \mathrm{E}+00$ & year & $1.04 E+08$ & $1.0736 \mathrm{E}+03$ & $9.3147 \mathrm{E}-04$ & & & \\
\hline $\mathrm{Rh} 101 \mathrm{~m}$ & 100.9062 & $4.340 E+00$ & day & $3.75 E+05$ & $2.9816 \mathrm{E}+05$ & $3.3539 E-06$ & & & \\
\hline $\mathrm{Rh} 102$ & 101.9068 & $2.070 E+02$ & day & $1.79 E+07$ & $6.1900 \mathrm{E}+03$ & $1.6155 \mathrm{E}-04$ & & $1.555 \mathrm{E}-09$ & $1.556 \mathrm{E}-09$ \\
\hline Rh $102 \mathrm{~m}$ & 101.9068 & $2.890 \mathrm{E}+00$ & year & $9.12 E+07$ & $1.2138 \mathrm{E}+03$ & $8.2384 E-04$ & & $8.645 \mathrm{E}-10$ & $8.645 \mathrm{E}-10$ \\
\hline Rh $103 \mathrm{~m}$ & 102.9055 & $5.612 E+01$ & minute & $3.37 \mathrm{E}+03$ & $3.2559 \mathrm{E}+07$ & $3.0714 \mathrm{E}-08$ & 0.000232 & $2.881 \mathrm{E}-11$ & $3.020 \mathrm{E}-02$ \\
\hline Rh 104 & 103.9067 & $4.230 \mathrm{E}+01$ & second & $4.23 E+01$ & $2.5668 \mathrm{E}+09$ & $3.8959 \mathrm{E}-10$ & & $1.839 \mathrm{E}-10$ & $7.377 \mathrm{E}-10$ \\
\hline Rh $104 \mathrm{~m}$ & 103.9067 & $4.340 \mathrm{E}+00$ & minute & $2.60 \mathrm{E}+02$ & $4.1696 \mathrm{E}+08$ & $2.3983 E-09$ & & $5.549 \mathrm{E}-10$ & $5.549 E-10$ \\
\hline Rh 105 & 104.9057 & $1.473 E+00$ & day & $1.27 E+05$ & $8.4501 E+05$ & 1.1834E-06 & & $1.475 \mathrm{E}-08$ & $9.638 \mathrm{E}-03$ \\
\hline Rh 105m & 104.9057 & $4.500 \mathrm{E}+01$ & second & $4.50 \mathrm{E}+01$ & $2.3898 E+09$ & $4.1844 \mathrm{E}-10$ & & $2.255 \mathrm{E}-09$ & $2.641 \mathrm{E}-03$ \\
\hline Rh 106 & 105.9073 & $2.980 \mathrm{E}+01$ & second & $2.98 \mathrm{E}+01$ & $3.5747 E+09$ & $2.7975 \mathrm{E}-10$ & 0.018940 & $5.169 \mathrm{E}-08$ & $4.023 \mathrm{E}-03$ \\
\hline $\mathrm{Rh} 106 \mathrm{~m}$ & 105.9073 & $2.183 E+00$ & hour & $7.86 E+03$ & $1.3553 E+07$ & $7.3785 \mathrm{E}-08$ & & $1.211 \mathrm{E}-07$ & $1.211 \mathrm{E}-07$ \\
\hline Rh 107 & 106.9068 & $2.170 E+01$ & minute & $1.30 \mathrm{E}+03$ & $8.1051 E+07$ & $1.2338 \mathrm{E}-08$ & & $1.21 .1 \mathrm{E}-07$ & $1.403 \mathrm{E}-03$ \\
\hline Rh 108 & 107.9087 & $1.680 \mathrm{E}+01$ & second & $1.68 \mathrm{E}+01$ & $6.2232 E+09$ & $1.6069 \mathrm{E}-10$ & & $3.368 \mathrm{E}-06$ & $6.614 \mathrm{E}-04$ \\
\hline Rh 108m & 107.9087 & $6.000 \mathrm{E}+00$ & minute & $3.60 \mathrm{E}+02$ & $2.9041 E+08$ & $3.4434 \mathrm{E}-09$ & & $7.889 \mathrm{E}-06$ & $7.889 \mathrm{E}-06$ \\
\hline
\end{tabular}

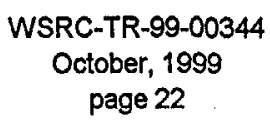


Radionuclide Specific Data

\begin{tabular}{|c|c|c|c|c|c|c|c|c|c|}
\hline Isotope & $\begin{array}{l}\text { Atomic } \\
\text { Mass }\end{array}$ & Halflife & $\begin{array}{l}\text { Time } \\
\text { Units }\end{array}$ & $t_{12}, \sec$ & Curies/gram & Grams/Curie & $\begin{array}{c}\text { Watts/Ci } \\
\text { (Ref.3) }\end{array}$ & \begin{tabular}{|} 
Individual $^{4}$ \\
Fission \\
Product Yield \\
(Ref.4)
\end{tabular} & $\begin{array}{l}\text { Cumulative }^{1} \\
\text { Fission Product } \\
\text { Yield (Ref.4) }\end{array}$ \\
\hline Rh 109 & 108.9087 & $1.333 E+00$ & minute & $8.00 E+01$ & $1.2952 E+09$ & $7.7209 E-10$ & & $2.891 \mathrm{E}-05$ & 3.443E-04 \\
\hline $\operatorname{Rh~} 110$ & 109.9110 & $3.200 E+00$ & second & $3.20 \mathrm{E}+00$ & $3.2076 \mathrm{E}+10$ & $3.1176 \mathrm{E}-11$ & & $1.562 \mathrm{E}-05$ & $2.650 \mathrm{E}-04$ \\
\hline $\mathrm{Rh} 110 \mathrm{~m}$ & 109.9110 & $2.850 E+01$ & second & $2.85 E+01$ & $3.6016 \mathrm{E}+09$ & $2.7766 \mathrm{E}-10$ & & $3.657 \mathrm{E}-05$ & $3.657 \mathrm{E}-05$ \\
\hline Rh 111 & 110.9117 & $1.100 E+01$ & second & $1.10 E+01$ & $9.2471 E+09$ & $1.0814 E-10$ & & $7.130 \mathrm{E}-05$ & $1.928 \mathrm{E}-04$ \\
\hline $\operatorname{Rh} 112$ & 111.9146 & $2.100 E+00$ & second & $2.10 \mathrm{E}+00$ & $4.8003 \mathrm{E}+10$ & $2.0832 E-11$ & & $7.220 \mathrm{E}-05$ & $1.406 \mathrm{E}-04$ \\
\hline Rh $112 \mathrm{~m}$ & 111.9146 & $6.800 \mathrm{E}+00$ & second & $6.80 E+00$ & $1.4825 \mathrm{E}+10$ & $6.7456 \mathrm{E}-11$ & & & \\
\hline Rh 113 & 112.8154 & $2.800 E+00$ & second & $2.80 E+00$ & $3.5683 \mathrm{E}+10$ & $2.8024 E-11$ & & $8.754 \mathrm{E}-05$ & $1.189 \mathrm{E}-04$ \\
\hline Rh 114 & 113.9188 & $1.850 E+00$ & second & $1.85 E+00$ & $5.3531 E+10$ & $1.8681 \mathrm{E}-11$ & & $5.608 \mathrm{E}-05$ & $6.717 \mathrm{E}-05$ \\
\hline Rh114m & 113.9188 & $1.850 E+00$ & second & $1.85 E+00$ & $5.3531 \mathrm{E}+10$ & $1.8681 \mathrm{E}-11$ & & & \\
\hline Rh 115 & 114.9201 & $9.900 \mathrm{E}-01$ & second & $9.90 \mathrm{E}-01$ & $9.9162 E+10$ & $1.0085 \mathrm{E}-11$ & & $2.938 \mathrm{E}-05$ & $3.126 \mathrm{E}-05$ \\
\hline$R h 116$ & 115.9237 & $6.800 \mathrm{E}-01$ & second & $6.80 \mathrm{E}-01$ & $1.4312 E+11$ & $6.9872 E-12$ & & $1.799 \mathrm{E}-05$ & $1.853 \mathrm{E}-05$ \\
\hline $\mathrm{Rh} 116 \mathrm{~m}$ & 115.9237 & $9.000 \mathrm{E}-01$ & second & $9.00 \mathrm{E}-01$ & $1.0813 E+11$ & $9.2478 E-12$ & & $5.259 \mathrm{E}-06$ & $5.308 \mathrm{E}-06$ \\
\hline $\operatorname{Rh~} 117$ & 116.9254 & $4.400 \mathrm{E}-01$ & second & $4.40 \mathrm{E}-01$ & $2.1929 E+11$ & $4.5602 E-12$ & & & \\
\hline $\mathrm{Pd} 95 \mathrm{~m}$ & 94.9247 & $1.330 \mathrm{E}+01$ & second & $1.33 E+01$ & $8.9361 \mathrm{E}+09$ & $1.1191 \mathrm{E}-10$ & & & \\
\hline$P d 96$ & 95.9182 & $1.220 E+02$ & second & $1.22 E+02$ & $9.6409 \mathrm{E}+08$ & $1.0373 E-09$ & & & \\
\hline$P d 97$ & 96.9165 & $3.100 E+00$ & minute & $1.86 E+02$ & $6.2584 E+08$ & $1.5978 E-09$ & & & \\
\hline$P d 98$ & 97.9127 & $1.770 E+01$ & minute & $1.06 E+03$ & $1.0850 \mathrm{E}+08$ & $9.2169 \mathrm{E}-09$ & & & \\
\hline Pd99 & 98.9118 & $2.140 E+01$ & minute & $1.28 E+03$ & $8.8831 E+07$ & $1.1257 \mathrm{E}-08$ & & & \\
\hline$P d 100$ & 99.9085 & $3.630 \mathrm{E}+00$ & day & $3.14 E+05$ & $3.6004 E+05$ & $2.7775 \mathrm{E}-06$ & & & \\
\hline Pd 101 & 100.9083 & $8.470 E+00$ & hour & $3.05 E+04$ & $3.6666 \mathrm{E}+06$ & $2.7273 \mathrm{E}-07$ & & & \\
\hline Pd 103 & 102.9061 & $1.699 \mathrm{E}+01$ & day & $1.47 E+06$ & $7.4680 \mathrm{E}+04$ & $1.3391 \mathrm{E}-05$ & & & \\
\hline$P d 107$ & 106.9051 & $6.500 E+06$ & year & $2.05 E+14$ & $5.1446 E-04$ & $1.9438 E+03$ & 0.000055 & $3.056 \mathrm{E}-10$ & $1.403 \mathrm{E}-03$ \\
\hline$P d 107 m$ & 106.9051 & $2.130 E+01$ & second & $2.13 E+01$ & $4.9545 \mathrm{E}+09$ & $2.0184 E-10$ & & $5.742 \mathrm{E}-10$ & $5.742 E-10$ \\
\hline Pd 109 & 108.9060 & $1.370 \mathrm{E}+01$ & hour & $4.93 E+04$ & $2.1002 E+06$ & $4.7614 \mathrm{E}-07$ & & $1.708 \mathrm{E}-07$ & $3.448 \mathrm{E}-04$ \\
\hline $\mathrm{Pd} 109 \mathrm{~m}$ & 108.9060 & $4.696 E+00$ & minute & $2.82 \mathrm{E}+02$ & $3.6766 \mathrm{E}+08$ & $2.7199 \mathrm{E}-09$ & & $3.211 \mathrm{E}-07$ & $3.210 \mathrm{E}-07$ \\
\hline$P d 111$ & 110.9076 & $2.340 E+01$ & minute & $1.40 \mathrm{E}+03$ & $7.2452 \mathrm{E}+07$ & $1.3802 \mathrm{E}-08$ & & $2.559 \mathrm{E}-06$ & $1.987 \mathrm{E}-04$ \\
\hline $\mathrm{Pd} 111 \mathrm{~m}$ & 110.9076 & $5.500 E+00$ & hour & $1.98 E+04$ & $5.1375 E+06$ & $1.9465 \mathrm{E}-07$ & & $4.809 E-06$ & $4.809 \mathrm{E}-06$ \\
\hline$P d 112$ & 111.9073 & $2.103 E+01$ & hour & $7.57 \mathrm{E}+04$ & $1.3316 E+06$ & $7.5097 E_{-07}$ & & $1.975 \mathrm{E}-05$ & $1.603 \mathrm{E}-04$ \\
\hline Pd 113 & 112.9102 & $1.550 E+00$ & minute & $9.30 \mathrm{E}+01$ & $1.0744 \mathrm{E}+09$ & $9.3076 \mathrm{E}-10$ & & $4.370 E-05$ & $1.626 \mathrm{E}-04$ \\
\hline $\mathrm{Pd} 113 \mathrm{~m}$ & 112.9102 & $1.000 E+02$ & second & $1.00 \mathrm{E}+02$ & $9.9918 \mathrm{E}+08$ & $1.0008 \mathrm{E}-09$ & & & \\
\hline $\mathrm{Pd} 113 \mathrm{~m} 2$ & 112.9102 & $4.000 \mathrm{E}-01$ & second & $4.00 \mathrm{E}-01$ & $2.4979 E+11$ & $4.0033 \mathrm{E}-12$ & & & \\
\hline$P d 114$ & 113.9104 & $2.420 E+00$ & minute & $1.45 E+02$ & $6.8210 \mathrm{E}+08$ & $1.4661 \mathrm{E}-09$ & & $7.086 \mathrm{E}-05$ & $1.381 \mathrm{E}-04$ \\
\hline$P d 115$ & 114.9137 & $2.500 E+01$ & second & $2.50 \mathrm{E}+01$ & $3.9270 \mathrm{E}+09$ & $2.5465 \mathrm{E}-10$ & & $7.288 \mathrm{E}-05$ & $1.041 \mathrm{E}-04$ \\
\hline$\overline{P d 115 m}$ & 114.9137 & $5.000 \mathrm{E}+01$ & second & $5.00 \mathrm{E}+01$ & $1.9635 \mathrm{E}+09$ & $5.0929 \mathrm{E}-10$ & & & \\
\hline$P d 116$ & 115.9142 & $1.240 E+01$ & second & $1.24 E+01$ & $7.8491 E+09$ & $1.2740 \mathrm{E}-10$ & & $1.254 \mathrm{E}-04$ & $1.440 \mathrm{E}-04$ \\
\hline
\end{tabular}

WSRC-TR-99-00344

October, 1999

page 23 
Radionuclide Specific Data

\begin{tabular}{|c|c|c|c|c|c|c|c|c|c|}
\hline Isotope & $\begin{array}{l}\text { Atomic } \\
\text { Mass }\end{array}$ & Halflife & $\begin{array}{l}\text { Time } \\
\text { Units }\end{array}$ & $t_{1 / 2}, \sec$ & Curies/ gram & Grams/ Curie & $\begin{array}{c}\text { Watts/Ci } \\
\text { (Ref.3) }\end{array}$ & $\begin{array}{c}\text { Individual }^{1} \\
\text { Fission } \\
\text { Product Yield } \\
\text { (Ref.4) }\end{array}$ & $\begin{array}{l}\text { Cumulative } \\
\text { Fission Product } \\
\text { Yield (Ret.4) }\end{array}$ \\
\hline $\mathrm{Pd} 117$ & 116.9178 & $4.300 E+00$ & second & $4.30 \mathrm{E}+00$ & $2.2440 E+10$ & $4.4563 \mathrm{E}-11$ & & $7.322 E-05$ & $7.840 E-05$ \\
\hline$P d 117 m$ & 116.9178 & $1.910 \mathrm{E}-02$ & second & $1.91 \mathrm{E}-02$ & $5.0520 \mathrm{E}+12$ & $1.9794 \mathrm{E}-13$ & & & \\
\hline Pd 118 & 117.9190 & $1.900 E+00$ & second & $1.90 E+00$ & $5.0355 E+10$ & $1.9859 \mathrm{E}-11$. & & $3.214 \mathrm{E}-05$ & $3.289 \mathrm{E}-05$ \\
\hline Pd 119 & 118.9227 & $9.200 \mathrm{E}-01$ & second & $9.20 \mathrm{E}-01$ & $1.0312 \mathrm{E}+11$ & $9.6979 \mathrm{E}-12$ & & $2.438 \mathrm{E}-05$ & $2.467 E-05$ \\
\hline $\mathrm{Ag} 96$ & 95.9307 & $5.100 \mathrm{E}+00$ & second & $5.10 \mathrm{E}+00$ & $2.3059 E+10$ & $4.3366 \mathrm{E}-11$ & & & \\
\hline Ag 97 & 96.9240 & $1.900 \mathrm{E}+01$ & second & $1.90 \mathrm{E}+01$ & $6.1262 E+09$ & $1.6323 \mathrm{E}-10$ & & & \\
\hline $\mathrm{Ag} 98$ & 97.9218 & $4.670 \mathrm{E}+01$ & second & $4.67 E+01$ & $2.4671 E+09$ & $4.0534 \mathrm{E}-10$ & & & \\
\hline $\operatorname{Ag} 99$ & 98.9176 & $1.240 E+02$ & second & $1.24 E+02$ & $9.1977 \mathrm{E}+08$ & $1.0872 \mathrm{E}-09$ & & & \\
\hline $\mathrm{Ag} 99 \mathrm{~m}$ & 98.9176 & $1.050 E+01$ & second & $1.05 E+01$ & $1.0862 \mathrm{E}+10$ & $9.2063 \mathrm{E}-11$ & & & \\
\hline $\mathrm{Ag} 100$ & 99.9161 & $2.010 E+00$ & minute & $1.21 E+02$ & $9.3625 \mathrm{E}+08$ & 1.0681E-09 & & & \\
\hline $\mathrm{Ag} 100 \mathrm{~m}$ & 99.9161 & $2.240 E+00$ & minute & $1.34 E+02$ & $8.4012 E+08$ & 1.1903E-09 & & & \\
\hline $\mathrm{Ag} 101$ & 100.9128 & $1.110 E+01$ & minute & $6.66 \mathrm{E}+02$ & $1.6786 E+08$ & $5.9572 \mathrm{E}-09$ & & & \\
\hline $\mathrm{Ag} 101 \mathrm{~m}$ & 100.9128 & $3.100 E+00$ & second & $3.10 \mathrm{E}+00$ & $3.6064 E+10$ & $2.7729 \mathrm{E}-11$ & & & \\
\hline $\mathrm{Ag} 102$ & 101.9120 & $1.290 E+01$ & minute & $7.74 E+02$ & $1.4302 E+08$ & $6.9918 E-09$ & & & \\
\hline $\mathrm{Ag} \mathrm{102m}$ & 101.9120 & $7.700 E+00$ & minute & $4.62 \mathrm{E}+02$ & $2.3961 \mathrm{E}+08$ & $4.1734 \mathrm{E}-09$ & & & \\
\hline $\mathrm{Ag} 103$ & 102.9090 & $1.095 E+00$ & hour & $3.94 \mathrm{E}+03$ & $2.7810 E+07$ & $3.5958 E-08$ & & & \\
\hline $\mathrm{Ag} \mathrm{103m}$ & 102.9090 & $5.700 \mathrm{E}+00$ & second & $5.70 \mathrm{E}+00$ & $1.9233 \mathrm{E}+10$ & $5.1994 \mathrm{E}-11$ & & & \\
\hline $\mathrm{Ag} 104$ & 103.9086 & $1.153 E+00$ & hour & $4.15 E+03$ & $2.6157 \mathrm{E}+07$ & $3.8230 \mathrm{E}-08$ & & & \\
\hline $\mathrm{Ag} 104 \mathrm{~m}$ & 103.9086 & $3.350 \mathrm{E}+01$ & minute & $2.01 E+03$ & $5.4017 \mathrm{E}+07$ & 1.8513E-08 & & & \\
\hline $\mathrm{Ag} 105$ & 104.9065 & $4.129 \mathrm{E}+01$ & day & $3.57 E+06$ & $3.0145 \mathrm{E}+04$ & $3.3173 \mathrm{E}-05$ & & & \\
\hline $\mathrm{Ag} 105 \mathrm{~m}$ & 104.9065 & $7.230 E+00$ & minute & $4.34 \mathrm{E}+02$ & $2.4790 E+08$ & $4.0338 E-09$ & & & \\
\hline $\mathrm{Ag} 106$ & 105.9067 & $2.396 E+01$ & minute & $1.44 E+03$ & $7.4099 E+07$ & $1.3495 \mathrm{E}-08$ & & & \\
\hline $\mathrm{Ag} 106 \mathrm{~m}$ & 105.9067 & $8.280 E+00$ & day. & $7.15 \mathrm{E}+05$ & $1.4890 \mathrm{E}+05$ & $6.7157 \mathrm{E}-06$ & & & \\
\hline $\mathrm{Ag} 107 \mathrm{~m}$ & 106.9051 & $4.430 E+01$ & second & $4.43 E+01$ & $2.3822 E+09$ & $4.1978 \mathrm{E}-10$ & & & \\
\hline $\mathrm{Ag} 108$ & 107.9060 & $2.370 E+00$ & minute & $1.42 E+02$ & $7.3524 \mathrm{E}+08$ & $1.3601 E-09$ & & & \\
\hline $\mathrm{Ag} \mathrm{108m}$ & 107.9060 & $4.180 \mathrm{E}+02$ & year & $1.32 \mathrm{E}+10$ & $7.9257 \mathrm{E}+00$ & $1.2617 \mathrm{E}-01$ & & & \\
\hline $\mathrm{Ag} \mathrm{109m}$ & 108.9048 & $3.960 E+01$ & second & $3.96 \mathrm{E}+01$ & $2.6160 E+09$ & $3.8227 \mathrm{E}-10$ & & & \\
\hline $\mathrm{Ag} 110$ & 109.9061 & $2.457 \mathrm{E}+01$ & second & $2.46 \mathrm{E}+01$ & $4.1778 E+09$ & $2.3936 \mathrm{E}-10$ & 0.007208 & $7.607 \mathrm{E}-10$ & $7.857 \mathrm{E}-10$ \\
\hline $\mathrm{Ag} 110 \mathrm{~m}$ & 109.9061 & $2.498 E+02$ & day & $2.16 \mathrm{E}+07$ & $4.7563 E+03$ & $2.1025 E-04$ & 0.016690 & $1.782 E-09$ & $1.782 E-09$ \\
\hline $\mathrm{Ag} 111$ & 110.9053 & $7.450 E+00$ & day & $6.44 E+05$ & $1.5804 E+05$ & $6.3277 \mathrm{E}-06$ & & $2.850 \mathrm{E}-09$ & $1.996 \mathrm{E}-04$ \\
\hline $\mathrm{Ag} 111 \mathrm{~m}$ & 110.9053 & $1.080 E+00$ & minute & $6.48 \mathrm{E}+01$ & $1.5698 E+09$ & $6.3702 E-10$ & & $1.865 \mathrm{E}-08$ & $1.986 \mathrm{E}-04$ \\
\hline $\mathrm{Ag} 112$ & 111.9070 & $3.130 \mathrm{E}+00$ & hour & $1.13 \mathrm{E}+04$ & $8.9469 E+06$ & 1.1177E-07 & & $1.250 \mathrm{E}-08$ & $1.604 \mathrm{E}-04$ \\
\hline $\mathrm{Ag} 113$ & 112.9066 & $5.370 \mathrm{E}+00$ & hour & $1.93 E+04$ & $5.1687 \mathrm{E}+06$ & 1.9347E-07 & & $9.766 E-08$ & $1.633 \mathrm{E}-04$ \\
\hline $\mathrm{Ag} \mathrm{113m}$ & 112.9066 & $1.145 \mathrm{E}+00$ & minute & $6.87 \mathrm{E}+01$ & $1.4545 \mathrm{E}+09$ & $6.8754 \mathrm{E}-10$ & & $6.390 \mathrm{E}-07$ & $6.390 \mathrm{E}-07$ \\
\hline $\mathrm{Ag} 114$ & 113.9088 & $4.600 \mathrm{E}+00$ & second & $4.60 \mathrm{E}+00$ & $2.1531 E+10$ & $4.6445 E-11$ & & $2.270 \mathrm{E}-06$ & $1.404 E-04$ \\
\hline
\end{tabular}

WSRC-TR-99-00344

October, 1999

page 24 


\begin{tabular}{|c|c|c|c|c|c|c|c|c|c|}
\hline Isotope & $\begin{array}{l}\text { Atomic } \\
\text { Mass }\end{array}$ & Halflife & $\begin{array}{l}\text { Time } \\
\text { Units }\end{array}$ & $t_{1 / 2}, \sec$ & Curies/ gram & Grams/ Curie & $\begin{array}{c}\text { Watts/Ci } \\
\text { (Ref.3) }\end{array}$ & $\begin{array}{c}\text { Individual }^{4} \\
\text { Fission }^{1} \\
\text { Product Yield } \\
\text { (Ref.4) }^{\text {Ref }}\end{array}$ & $\begin{array}{c}\text { Cumulative }^{\natural} \\
\text { Fission Product } \\
\text { Yield (Ref.4) }\end{array}$ \\
\hline$\overline{\mathrm{Ag} 115}$ & 114.9088 & $2.000 E+01$ & minute & $1.20 \mathrm{E}+03$ & $8.1817 \mathrm{E}+07$ & $1.2222 E-08$ & & $9.570 \mathrm{E}-07$ & $7.698 \mathrm{E}-05$ \\
\hline $\mathrm{Ag} \mathrm{115m}$ & 114.9088 & $1.800 \mathrm{E}+01$ & second & $1.80 \mathrm{E}+01$ & $5.4544 E+09$ & $1.8334 \mathrm{E}-10$ & & $6.261 \mathrm{E}-06$ & $3.438 \mathrm{E}-05$ \\
\hline $\mathrm{Ag} 116$ & 115.9114 & $2.680 E+00$ & minute & $1.61 \mathrm{E}+02$ & $6.0529 E+08$ & $1.6521 \mathrm{E}-09$ & & $4.629 \mathrm{E}-06$ & $1.491 \mathrm{E}-04$ \\
\hline $\mathrm{Ag} 116 \mathrm{~m}$ & 115.9114 & $1.040 \mathrm{E}+01$ & second & $1.04 E+01$ & $9.3587 E+09$ & $1.0685 \mathrm{E}-10$ & & $1.947 \mathrm{E}-05$ & 1.947E-05 \\
\hline $\mathrm{Ag} 117$ & 116.9117 & $1.213 \mathrm{E}+00$ & minute & $7.28 \mathrm{E}+01$ & $1.3256 E+09$ & $7.5440 \mathrm{E}-10$ & & $3.422 \mathrm{E}-06$ & $8.182 E-05$ \\
\hline $\mathrm{Ag} 117 \mathrm{~m}$ & 116.9117 & $5.340 E+00$ & second & $5.34 \mathrm{E}+00$ & $1.8071 \mathrm{E}+10$ & $5.5338 \mathrm{E}-11$ & & $2.239 \mathrm{E}-05$ & $2.239 E-05$ \\
\hline $\mathrm{Ag} 118$ & 117.9146 & $3.760 E+00$ & second & $3.76 E+00$ & $2.5446 E+10$ & $3.9299 \mathrm{E}-11$ & & $1.199 \mathrm{E}-05$ & $6.554 \mathrm{E}-05$ \\
\hline $\mathrm{Ag} \mathrm{118m}$ & 117.9146 & $2.000 \mathrm{E}+00$ & second & $2.00 \mathrm{E}+00$ & $4.7839 E+10$ & $2.0904 \mathrm{E}-11$ & & $5.040 \mathrm{E}-05$ & $5.040 \mathrm{E}-05$ \\
\hline $\operatorname{Ag} 119$ & 118.9157 & $2.100 \mathrm{E}+00$ & second & $2.10 E+00$ & $4.5177 \mathrm{E}+10$ & $2.2135 \mathrm{E}-11$ & & $6.539 \mathrm{E}-05$ & $9.006 \mathrm{E}-05$ \\
\hline $\mathrm{Ag} \mathrm{119m}$ & 118.9157 & $6.000 \mathrm{E}+00$ & second & $6.00 \mathrm{E}+00$ & $1.5812 \mathrm{E}+10$ & $6.3243 \mathrm{E}-11$ & & & \\
\hline $\operatorname{Ag} 120$ & 119.9188 & $1.230 E+00$ & second & $1.23 E+00$ & $7.6486 \mathrm{E}+10$ & $1.3074 \mathrm{E}-11$ & & $2.022 E-05$ & $4.076 \mathrm{E}-05$ \\
\hline $\mathrm{Ag} \mathrm{120m}$ & 119.9188 & $3.200 \mathrm{E}-01$ & second & $3.20 \mathrm{E}-01$ & $2.9399 \mathrm{E}+11$ & $3.4014 \mathrm{E}-12$ & & $2.869 E-05$ & $2.869 E-05$ \\
\hline $\operatorname{Ag} 121$ & 120.9199 & $7.800 \mathrm{E}-01$ & second & $7.80 \mathrm{E}-01$ & $1.1961 \mathrm{E}+11$ & $8.3602 \mathrm{E}-12$ & & $3.818 \mathrm{E}-05$ & $4.107 \mathrm{E}-05$ \\
\hline $\mathrm{Ag} 122$ & 121.9233 & $4.800 \mathrm{E}-01$ & second & $4.80 \mathrm{E}-01$ & $1.9277 E+11$ & $5.1874 \mathrm{E}-12$ & & $2.097 \mathrm{E}-05$ & $2.180 \mathrm{E}-05$ \\
\hline $\mathrm{Ag} \mathrm{122m}$ & 121.9233 & $1.500 \mathrm{E}+00$ & second & $1.50 \mathrm{E}+00$ & $6.1688 \mathrm{E}+10$ & $1.6211 \mathrm{E}-11$ & & & \\
\hline $\mathrm{Ag} 123$ & 122.9249 & $3.090 \mathrm{E}-01$ & second & $3.09 \mathrm{E}-01$ & $2.9701 \mathrm{E}+11$ & $3.3668 \mathrm{E}-12$ & & $6.769 \mathrm{E}-06$ & $6.846 \mathrm{E}-06$ \\
\hline Cd 97 & 96.9349 & $3.000 \mathrm{E}+00$ & second & $3.00 E+00$ & $3.8795 E+10$ & $2.5777 \mathrm{E}-11$ & & & \\
\hline Cd 98 & 97.9276 & $9.200 \mathrm{E}+00$ & second & $9.20 \mathrm{E}+00$ & $1.2522 E+10$ & $7.9858 \mathrm{E}-11$ & & & \\
\hline Cd 99 & 98.9250 & $1.600 \mathrm{E}+01$ & second & $1.60 \mathrm{E}+01$ & $7.1277 E+09$ & $1.4030 \mathrm{E}-10$ & & & \\
\hline Cd 100 & 99.9202 & $4.910 E+01$ & second & $4.91 E+01$ & $2.2995 \mathrm{E}+09$ & $4.3487 \mathrm{E}-10$ & & & \\
\hline Cd 101 & 100.9187 & $1.200 E+00$ & minute & $7.20 \mathrm{E}+01$ & $1.5526 \mathrm{E}+09$ & $6.4406 \mathrm{E}-10$ & & & \\
\hline Cd 102 & 101.9148 & $5.500 E+00$ & minute & $3.30 \mathrm{E}+02$ & $3.3545 \mathrm{E}+08$ & $2.9811 \mathrm{E}-09$ & & & \\
\hline Cd 103 & 102.9134 & $7.300 E+00$ & minute & $4.38 E+02$ & $2.5028 E+08$ & 3.9955E-09 & & & \\
\hline Cd 104 & 103.9098 & $5.770 E+01$ & minute & $3.46 \mathrm{E}+03$ & $3.1361 \mathrm{E}+07$ & $3.1887 \mathrm{E}-08$ & & & \\
\hline Cd 105 & 104.9095 & $5.550 \mathrm{E}+01$ & minute & $3.33 E+03$ & $3.2294 \mathrm{E}+07$ & $3.0966 \mathrm{E}-08$ & & & \\
\hline Cd 107 & 106.9066 & $6.500 E+00$ & hour & $2.34 E+04$ & $4.5098 E+06$ & $2.2174 \mathrm{E}-07$ & & & \\
\hline Cd 109 & 108.9050 & $4.626 \mathrm{E}+02$ & day & $4.00 \mathrm{E}+07$ & $2.5918 \mathrm{E}+03$ & $3.8583 \mathrm{E}-04$ & & & $1.010 \mathrm{E}-14$ \\
\hline $\mathrm{Cd} 111 \mathrm{~m}$ & 110.9042 & $4.854 \mathrm{E}+01$ & minute & $2.91 \mathrm{E}+03$ & $3.4928 \mathrm{E}+07$ & $2.8630 \mathrm{E}-08$ & & & $4.985 \mathrm{E}-12$ \\
\hline Cd 113 & 112.9044 & $9.300 E+15$ & year & $2.93 E+23$ & $3.4046 \mathrm{E}-13$ & $2.9372 E+12$ & 0.000541 & & $1.607 \mathrm{E}-04$ \\
\hline $\mathrm{Cd} 113 \mathrm{~m}$ & 112.9044 & $1.410 \mathrm{E}+01$ & year & $4.45 E+08$ & $2.2456 \mathrm{E}+02$ & $4.4532 \mathrm{E}-03$ & 0.001080 & & $2.614 \mathrm{E}-06$ \\
\hline $\begin{array}{ll}\operatorname{Cd} 115 \\
\end{array}$ & 114.9054 & $2.228 \mathrm{E}+00$ & day & $1.92 E+05$ & $5.1016 \mathrm{E}+05$ & $1.9602 \mathrm{E}-06$ & & & $1.025 \mathrm{E}-04$ \\
\hline Cd $115 \mathrm{~m}$ & 114.9054 & $4.460 E+01$ & day & $3.85 \mathrm{E}+06$ & $2.5479 \mathrm{E}+04$ & $3.9248 \mathrm{E}-05$ & 0.003760 & & $8.940 \mathrm{E}-06$ \\
\hline $\begin{array}{ll}\operatorname{Cd} 117 \\
\end{array}$ & 116.9072 & $2.490 \mathrm{E}+00$ & hour & $8.96 E+03$ & $1.0765 E+07$ & $9.2890 \mathrm{E}-08$ & & & $9.041 \mathrm{E}-05$ \\
\hline $\mathrm{Cd} 117 \mathrm{~m}$ & 116.9072 & $3.360 \mathrm{E}+00$ & hour & $1.21 E+04$ & $7.9780 E+06$ & $1.2534 \mathrm{E}-07$ & & & $1.792 E-05$ \\
\hline Cd 118 & 117.9069 & $5.030 \mathrm{E}+01$ & minute & $3.02 E+03$ & $3.1704 E+07$ & $3.1541 \mathrm{E}-08$ & & & $1.093 \mathrm{E}-04$ \\
\hline
\end{tabular}


Radionuclide Specific Data

\begin{tabular}{|c|c|c|c|c|c|c|c|c|c|}
\hline |sotope & $\begin{array}{l}\text { Atomic } \\
\text { Mass }\end{array}$ & Halflife & $\begin{array}{l}\text { Time } \\
\text { Units }\end{array}$ & $t_{1 / 2}, \sec$ & Curies/ gram & Grams/ Curie & $\begin{array}{c}\text { Watts/Ci } \\
\text { (Ref.3) }\end{array}$ & $\begin{array}{c}\text { Individual }^{1} \\
\text { Fission } \\
\text { Product Yield } \\
\text { (Ref.4) }^{-4} \\
\end{array}$ & $\begin{array}{c}\text { Cumulative' } \\
\text { Fission Product } \\
\text { Yield (Ref.4) }\end{array}$ \\
\hline Cd 119 & 118.9099 & $2.690 \mathrm{E}+00$ & minute & $1.61 \mathrm{E}+02$ & $5.8783 E+08$ & $1.7012 E-09$ & & & $8.003 E-05$ \\
\hline Cd $119 m$ & 118.9099 & $2.200 \mathrm{E}+00$ & minute & $1.32 \mathrm{E}+02$ & $7.1876 \mathrm{E}+08$ & $1.3913 \mathrm{E}-09$ & & & 4.108E-05 \\
\hline Cd 120 & 119.9099 & $5.080 \mathrm{E}+01$ & second & $5.08 E+01$ & $1.8521 \mathrm{E}+09$ & $5.3994 \mathrm{E}-10$ & & & $1.192 E-04$ \\
\hline Cd 121 & 120.9130 & $1.350 \mathrm{E}+01$ & second & $1.35 E+01$ & $6.9115 \mathrm{E}+09$ & $1.4469 \mathrm{E}-10$ & & & $6.496 \mathrm{E}-05$ \\
\hline Cd $121 \mathrm{~m}$ & 120.9130 & $8.300 E+00$ & second & $8.30 \mathrm{E}+00$ & $1.1242 \mathrm{E}+10$ & $8.8956 \mathrm{E}-11$ & & & $5.780 \mathrm{E}-05$ \\
\hline Cd 122 & 121.9135 & $5.240 \mathrm{E}+00$ & second & $5.24 \mathrm{E}+00$ & $1.7660 \mathrm{E}+10$ & $5.6625 \mathrm{E}-11$ & & & $1.355 \mathrm{E}-04$ \\
\hline Cd 123 & 122.9170 & $2.100 \mathrm{E}+00$ & second & $2.10 \mathrm{E}+00$ & $4.3706 \mathrm{E}+10$ & $2.2880 \mathrm{E}-11$ & & & $8.529 \mathrm{E}-05$ \\
\hline $\mathrm{Cd} 123 \mathrm{~m}$ & 122.9170 & $1.820 \mathrm{E}+00$ & second & $1.82 \mathrm{E}+00$ & $5.0430 \mathrm{E}+10$ & $1.9829 \mathrm{E}-11$ & & & \\
\hline Cd 124 & 123.9176 & $1.250 \mathrm{E}+00$ & second & $1.25 \mathrm{E}+00$ & $7.2834 \mathrm{E}+10$ & $1.3730 \mathrm{E}-11$ & & & $1.618 E-04$ \\
\hline $\mathrm{Cd} 125$ & 124.9212 & $6.500 \mathrm{E}-01$ & second & $6.50 \mathrm{E}-01$ & $1.3894 \mathrm{E}+11$ & $7.1974 \mathrm{E}-12$ & & & $8.974 \mathrm{E}-05$ \\
\hline Cd 125m & 124.9212 & $5.700 \mathrm{E}-01$ & second & $5.70 \mathrm{E}-01$ & $1.5844 \mathrm{E}+11$ & $6.3115 E-12$ & & & \\
\hline Cd 126 & 125.9224 & $5.070 E-01$ & second & 5.07E-01 & $1.7671 \mathrm{E}+11$ & $5.6589 \mathrm{E}-12$ & & & $1.162 \mathrm{E}-04$ \\
\hline Cd 127 & 126.9264 & 4.300E-01 & second & 4.30E-01 & $2.0671 E+11$ & $4.8378 \mathrm{E}-12$ & & & $8.513 \mathrm{E}-05$ \\
\hline Cd 128 & 127.9278 & $3.400 \mathrm{E}-01$ & second & $3.40 \mathrm{E}-01$ & $2.5938 \mathrm{E}+11$ & $3.8554 \mathrm{E}-12$ & & & $6.363 E-05$ \\
\hline Cd 129 & 128.9323 & $2.700 \mathrm{E}-01$ & second & $2.70 \mathrm{E}-01$ & $3.2408 \mathrm{E}+11$ & $3.0857 \mathrm{E}-12$ & & & $2.160 \mathrm{E}-05$ \\
\hline Cd 130 & 129.9340 & $2.000 \mathrm{E}-01$ & second & $2.00 E-01$ & $4.3413 \mathrm{E}+11$ & $2.3034 \mathrm{E}-12$ & & & $9.169 \mathrm{E}-06$ \\
\hline $\ln 101$ & 100.9266 & $1.600 E+01$ & second & $1.60 \mathrm{E}+01$ & $6.9864 E+09$ & $1.4314 \mathrm{E}-10$ & & & \\
\hline $\ln 102$ & 101.9247 & $2.400 E+01$ & second & $2.40 E+01$ & $4.6120 E+09$ & $2.1683 \mathrm{E}-10$ & & & \\
\hline $\ln 103$ & 102.9199 & $6.500 \mathrm{E}+01$ & second & $6.50 \mathrm{E}+01$ & $1.6864 \mathrm{E}+09$ & $5.9297 \mathrm{E}-10$ & & & \\
\hline $\ln 104$ & 103.9183 & $1.800 \mathrm{E}+00$ & minute & $1.08 E+02$ & $1.0052 E+09$ & $9.9481 \mathrm{E}-10$ & & & \\
\hline In $104 m$ & 103.9183 & $1.570 \mathrm{E}+01$ & second & $1.57 E+01$ & $6.9149 \mathrm{E}+09$ & $1.4462 E-10$ & & & \\
\hline $\ln 105$ & 104.9147 & $5.070 E+00$ & minute & $3.04 \mathrm{E}+02$ & $3.5349 E+08$ & $2.8289 \mathrm{E}-09$ & & & \\
\hline In $105 m$ & 104.9147 & $4.800 \mathrm{E}+01$ & second & $4.80 \mathrm{E}+01$ & $2.2403 E+09$ & $4.4638 \mathrm{E}-10$ & & & \\
\hline $\ln 106$ & 105.9135 & $6.200 E+00$ & minute & $3.72 E+02$ & $2.8634 E+08$ & $3.4923 \mathrm{E}-09$ & & & \\
\hline In $106 \mathrm{~m}$ & 105.9135 & $5.200 \mathrm{E}+00$ & minute & $3.12 E+02$ & $3.4141 E+08$ & $2.9291 \mathrm{E}-09$ & & & \\
\hline $\ln 107$ & 106.9103 & $3.240 E+01$ & minute & $1.94 \mathrm{E}+03$ & $5.4283 \mathrm{E}+07$ & $1.8422 \mathrm{E}-08$ & & & \\
\hline In $107 \mathrm{~m}$ & 106.9103 & $5.040 E+01$ & second & $5.04 E+01$ & $2.0938 \mathrm{E}+09$ & $4.7761 \mathrm{E}-10$ & & & \\
\hline $\ln 108$ & 107.9097 & $5.800 E+01$ & minute & $3.48 \mathrm{E}+03$ & $3.0043 E+07$ & $3.3286 \mathrm{E}-08$ & & & \\
\hline In $108 \mathrm{~m}$ & 107.9097 & $3.960 \mathrm{E}+01$ & minute & $2.38 E+03$ & $4.4002 E+07$ & $2.2726 \mathrm{E}-08$ & & & \\
\hline $\ln 109$ & 108.9072 & $4.200 \mathrm{E}+00$ & hour & $1.51 \mathrm{E}+04$ & $6.8512 E+06$ & $1.4596 \mathrm{E}-07$ & & & \\
\hline In $109 \mathrm{~m}$ & 108.9072 & $1.340 E+00$ & minute & $8.04 E+01$ & 1.2884E+09 & $7.7613 \mathrm{E}-10$ & & & \\
\hline $\ln 110$ & 109.9072 & $4.900 E+00$ & hour & 1.76E+04 & $5.8190 \mathrm{E}+06$ & 1.7185E-07 & & & \\
\hline In $110 \mathrm{~m}$ & 109.9072 & $1.152 \mathrm{E}+00$ & hour & $4.15 E+03$ & $2.4751 \mathrm{E}+07$ & $4.0402 \mathrm{E}-08$ & & & \\
\hline $\ln 111$ & 110.9051 & $2.805 E+00$ & day & $2.42 E+05$ & 4.1975E+05 & $2.3824 \mathrm{E}-06$ & 0.002604 & & \\
\hline In $111 \mathrm{~m}$ & 110.9051 & $7.700 \mathrm{E}+00$ & minute & $4.62 E+02$ & $2.2018 \mathrm{E}+08$ & $4.5417 \mathrm{E}-09$ & & & \\
\hline
\end{tabular}

WSRC-TR-99-00344

October, 1999

page 26 


\begin{tabular}{|c|c|c|c|c|c|c|c|c|c|}
\hline Isotope & $\begin{array}{c}\text { Atomic } \\
\text { Mass }\end{array}$ & Halflife & $\begin{array}{l}\text { Time } \\
\text { Units }\end{array}$ & $t_{1 / 2}, \sec$ & Curies/ gram & Grams/Curie & $\begin{array}{c}\text { Watts/Ci } \\
\text { (Ref.3) }\end{array}$ & $\begin{array}{c}\text { Individual }^{1} \\
\text { Fission } \\
\text { Product Yield } \\
\text { (Ref.4) } \\
\end{array}$ & $\begin{array}{c}\text { Cumulative } \\
\text { Fission Product } \\
\text { Yield (Ref.4) }\end{array}$ \\
\hline $\ln 112$ & 111.9055 & $1.497 E+01$ & minute & $8.98 \mathrm{E}+02$ & $1.1224 E+08$ & $8.9094 \mathrm{E}-09$ & & & \\
\hline $\ln 112 m$ & 111.9055 & $2.056 \mathrm{E}+01$ & minute & $1.23 E+03$ & $8.1724 \mathrm{E}+07$ & $1.2236 \mathrm{E}-08$ & & & \\
\hline $\ln 113 m$ & 112.9041 & $1.658 \mathrm{E}+00$ & hour & $5.97 \mathrm{E}+03$ & $1.6739 \mathrm{E}+07$ & $5.9741 \mathrm{E}-08$ & 0.002310 & & \\
\hline $\ln 114$ & 113.9049 & $1.198 \mathrm{E}+00$ & minute & $7.19 \mathrm{E}+01$ & $1.3779 \mathrm{E}+09$ & $7.2573 \mathrm{E}-10$ & & & \\
\hline In $114 m$ & 113.9049 & $4.951 \mathrm{E}+01$ & day & $4.28 E+06$ & $2.3154 E+04$ & $4.3189 \mathrm{E}-05$ & 0.001400 & & \\
\hline $\ln 115$ & 114.9039 & $4.410 E+14$ & year & $1.39 E+22$ & $7.0548 \mathrm{E}-12$ & $1.4175 \mathrm{E}+11$ & & & $1.076 \mathrm{E}-04$ \\
\hline $\operatorname{In} 115 \mathrm{~m}$ & 114.9039 & $4.486 E+00$ & hour & $1.61 E+04$ & $6.0797 E+06$ & $1.6448 \mathrm{E}-07$ & & & $1.025 \mathrm{E}-04$ \\
\hline $\ln 116$ & 115.9053 & $1.410 E+01$ & second & $1.41 \mathrm{E}+01$ & $6.9033 \mathrm{E}+09$ & $1.4486 \mathrm{E}-10$ & & & $1.361 \mathrm{E}-10$ \\
\hline $\ln 116 m$ & 115.9053 & $5.441 \mathrm{E}+01$ & minute & $3.26 \mathrm{E}+03$ & $2.9816 \mathrm{E}+07$ & $3.3540 \mathrm{E}-08$ & & & $6.037 \mathrm{E}-10$ \\
\hline $\ln 116 \mathrm{~m} 2$ & 115.9053 & $2.180 E+00$ & second & $2.18 E+00$ & $4.4650 \mathrm{E}+10$ & $2.2397 \mathrm{E}-11$ & & & \\
\hline $\ln 117$ & 116.9045 & $4.320 \mathrm{E}+01$ & minute & $2.59 E+03$ & $3.7231 \mathrm{E}+07$ & $2.6859 \mathrm{E}-08$ & & & $6.435 \mathrm{E}-05$ \\
\hline $\ln 117 m$ & 116.9045 & $1.162 \mathrm{E}+02$ & minute & $6.97 E+03$ & $1.3842 E+07$ & $7.2246 \mathrm{E}-08$ & & & $8.300 \mathrm{E}-05$ \\
\hline $\ln 118$ & 117.9064 & $5.000 \mathrm{E}+00$ & second & $5.00 \mathrm{E}+00$ & $1.9137 \mathrm{E}+10$ & $5.2255 \mathrm{E}-11$ & & & $1.093 \mathrm{E}-04$ \\
\hline $\ln 118 \mathrm{~m}$ & 117.9064 & $4.450 \mathrm{E}+00$ & minute & $2.67 \mathrm{E}+02$ & $3.5837 \mathrm{E}+08$ & $2.7904 \mathrm{E}-09$ & & & $6.414 \mathrm{E}-08$ \\
\hline $\ln 118 \mathrm{~m} 2$ & 117.9064 & $8.500 E+00$ & second & $8.50 E+00$ & $1.1257 \mathrm{E}+10$ & $8.8834 \mathrm{E}-11$ & & & \\
\hline $\ln 119$ & 118.9058 & $2.400 E+00$ & minute & $1.44 E+02$ & $6.5889 \mathrm{E}+08$ & $1.5177 \mathrm{E}-09$ & & & $5.095 \mathrm{E}-05$ \\
\hline $\ln 119 m$ & 118.9058 & $1.800 \mathrm{E}+01$ & minute & $1.08 E+03$ & $8.7852 E+07$ & 1.1383E-08 & & & $7.438 \mathrm{E}-05$ \\
\hline $\ln 120$ & 119.9080 & $3.080 \mathrm{E}+00$ & second & $3.08 \mathrm{E}+00$ & $3.0548 \mathrm{E}+10$ & $3.2736 \mathrm{E}-11$ & & & 1.196E-04 \\
\hline $\ln 120 \mathrm{~m}$ & 119.9080 & $4.620 \mathrm{E}+01$ & second & $4.62 \mathrm{E}+01$ & $2.0365 \mathrm{E}+09$ & $4.9104 \mathrm{E}-10$ & & & $1.409 \mathrm{E}-06$ \\
\hline $\ln 120 \mathrm{~m} 2$ & 119.9080 & $4.730 E+01$ & second & $4.73 E+01$ & $1.9891 E+09$ & $5.0273 E-10$ & & & \\
\hline $\ln 121$ & 120.9078 & $2.310 E+01$ & second & $2.31 \mathrm{E}+01$ & $4.0393 E+09$ & $2.4757 \mathrm{E}-10$ & & & $6.412 \mathrm{E}-05$ \\
\hline $\ln 121 \mathrm{~m}$ & 120.9078 & $3.880 \mathrm{E}+00$ & minute & $2.33 \mathrm{E}+02$ & $4.0081 E+08$ & $2.4949 \mathrm{E}-09$ & & & $6.623 \mathrm{E}-05$ \\
\hline $\ln 122$ & 121.9103 & $1.500 \mathrm{E}+00$ & second & $1.50 \mathrm{E}+00$ & $6.1694 \mathrm{E}+10$ & $1.6209 \mathrm{E}-11$ & & & 1.387E-04 \\
\hline $\ln 122 m$ & 121.9103 & $1.030 \mathrm{E}+01$ & second & $1.03 \mathrm{E}+01$ & $8.9846 \mathrm{E}+09$ & $1.1130 \mathrm{E}-10$ & & & 1.371E-05 \\
\hline $\ln 122 \mathrm{~m} 2$ & 121.9103 & $1.080 E+01$ & second & $1.08 \mathrm{E}+01$ & $8.5687 \mathrm{E}+09$ & $1.1670 \mathrm{E}-10$ & & & \\
\hline $\ln 123$ & 122.9104 & $5.980 \mathrm{E}+00$ & second & $5.98 E+00$ & $1.5349 \mathrm{E}+10$ & $6.5150 \mathrm{E}-11$ & & & 1.141E-04 \\
\hline $\ln 123 m$ & 122.9104 & $4.780 \mathrm{E}+01$ & second & $4.78 \mathrm{E}+01$ & $1.9203 E+09$ & $5.2076 \mathrm{E}-10$ & & & $6.653 \mathrm{E}-06$ \\
\hline $\ln 124$ & 123.9132 & $3.170 \mathrm{E}+00$ & second & $3.17 E+00$ & $2.8721 E+10$ & $3.4818 \mathrm{E}-11$ & & & 1.874E-04 \\
\hline \begin{tabular}{|l|}
$\ln 124 m$ \\
\end{tabular} & 123.9132 & $2.400 \mathrm{E}+00$ & second & $2.40 \mathrm{E}+00$ & $3.7936 \mathrm{E}+10$ & $2.6360 \mathrm{E}-11$ & & & $6.014 \mathrm{E}-05$ \\
\hline $\ln 125$ & 124.9136 & $2.360 \mathrm{E}+00$ & second & $2.36 \mathrm{E}+00$ & $3.8270 \mathrm{E}+10$ & $2.6130 \mathrm{E}-11$ & & & $1.845 \mathrm{E}-04$ \\
\hline $\ln 125 \mathrm{~m}$ & 124.9136 & $1.220 \mathrm{E}+01$ & second & $1.22 \mathrm{E}+01$ & $7.4030 \mathrm{E}+09$ & $1.3508 \mathrm{E}-10$ & & & $2.190 \mathrm{E}-05$ \\
\hline $\ln 126$ & 125.9165 & $1.600 \mathrm{E}+00$ & second & $1.60 \mathrm{E}+00$ & $5.5998 \mathrm{E}+10$ & $1.7858 \mathrm{E}-11$ & & & $2.026 \mathrm{E}-04$ \\
\hline $\ln 126 \mathrm{~m}$ & 125.9165 & $1.640 \mathrm{E}+00$ & second & $1.64 \mathrm{E}+00$ & $5.4632 \mathrm{E}+10$ & $1.8304 \mathrm{E}-11$ & & & $1.834 \mathrm{E}-04$ \\
\hline $\ln 127$ & 126.9173 & $1.090 \mathrm{E}+00$ & second & $1.09 \mathrm{E}+00$ & $8.1551 \mathrm{E}+10$ & $1.2262 \mathrm{E}-11$ & & & $4.610 \mathrm{E}-04$ \\
\hline $\ln 127 m$ & 126.9173 & $3.670 E+00$ & second & $3.67 \mathrm{E}+00$ & $2.4221 E+10$ & $4.1287 \mathrm{E}-11$ & & & $1.557 \mathrm{E}-04$ \\
\hline
\end{tabular}




\begin{tabular}{|c|c|c|c|c|c|c|c|c|c|}
\hline Isotope & $\begin{array}{c}\text { Atomic } \\
\text { Mass }\end{array}$ & Halflife & $\begin{array}{l}\text { Time } \\
\text { Units }\end{array}$ & $t_{12}, \sec$ & Curies/gram & Grams/ Curie & $\begin{array}{c}\text { Watts/Ci } \\
\text { (Ref.3) }\end{array}$ & $\begin{array}{c}\text { Individual }^{1} \\
\text { Fission } \\
\text { Product Yield } \\
\text { (Ref.4) } \\
\end{array}$ & $\begin{array}{c}\text { Cumulative } \\
\text { Fission Product } \\
\text { Yield (Ref.4) }\end{array}$ \\
\hline $\ln 128$ & 127.9202 & $8.400 E-01$ & second & $8.40 \mathrm{E}-01$ & $1.0499 \mathrm{E}+11$ & $9.5245 E-12$ & & & 2.217E-04 \\
\hline $\ln 128 m$ & 127.9202 & $7.200 \mathrm{E}-01$ & second & $7.20 \mathrm{E}-01$ & $1.2249 E+11$ & $8.1639 \mathrm{E}-12$ & & & $6.647 \mathrm{E}-04$ \\
\hline $\ln 129$ & 128.9217 & $6.100 E-01$ & second & $6.10 \mathrm{E}-01$ & $1.4346 E+11$ & $6.9707 E-12$ & & & $7.674 E-04$ \\
\hline $\ln 129 m$ & 128.9217 & $1.230 E+00$ & second & $1.23 E+00$ & $7.1145 E+10$ & $1.4056 \mathrm{E}-11$ & & & $1.725 \mathrm{E}-04$ \\
\hline $\ln 130$ & 129.9249 & $2.600 \mathrm{E}-01$ & second & $2.60 \mathrm{E}-01$ & $3.3397 \mathrm{E}+11$ & $2.9943 \mathrm{E}-12$ & & & 9.487E-04 \\
\hline $\ln 130 \mathrm{~m}$ & 129.9249 & $5.500 \mathrm{E}-01$ & second & $5.50 \mathrm{E}-01$ & $1.5788 \mathrm{E}+11$ & $6.3340 E-12$ & & & \\
\hline $\ln 130 \mathrm{~m} 2$ & 129.9249 & $5.500 \mathrm{E}-01$ & second & $5.50 \mathrm{E}-01$ & $1.5788 \mathrm{E}+11$ & $6.3340 \mathrm{E}-12$ & & & \\
\hline $\ln 131$ & 130.9268 & $2.820 \mathrm{E}-01$ & second & $2.82 E-01$ & $3.0556 E+11$ & $3.2727 \mathrm{E}-12$ & & & $1.785 E-04$ \\
\hline $\ln 131 m$ & 130.9268 & $3.500 E+01$ & second & $3.50 E+01$ & $2.4620 E+09$ & $4.0618 \mathrm{E}-10$ & & & $4.110 \mathrm{E}-05$ \\
\hline $\ln 131 \mathrm{~m} 2$ & 130.9268 & $3.200 E-01$ & second & $3.20 \mathrm{E}-01$ & $2.6928 \mathrm{E}+11$ & $3.7137 E-12$ & & & \\
\hline $\ln 132$ & 131.9329 & $2.010 \mathrm{E}-01$ & second & $2.01 \mathrm{E}-01$ & $4.2543 E+11$ & $2.3506 \mathrm{E}-12$ & & & $6.747 \mathrm{E}-05$ \\
\hline $\ln 133$ & 132.9383 & $1.800 \mathrm{E}-01$ & second & $1.80 \mathrm{E}-01$ & $4.7147 \mathrm{E}+11$ & $2.1210 E-12$ & & & 3.987E-07 \\
\hline $\operatorname{Sn} 103$ & 102.9281 & $7.000 E+00$ & second & $7.00 E+00$ & $1.5658 E+10$ & $6.3864 E-11$ & & & \\
\hline Sn 104 & 103.9232 & $2.080 E+01$ & second & $2.08 E+01$ & $5.2192 \mathrm{E}+09$ & $1.9160 \mathrm{E}-10$ & & & \\
\hline Sn 105 & 104.9214 & $3.100 E+01$ & second & $3.10 E+01$ & $3.4686 E+09$ & $2.8830 \mathrm{E}-10$ & & & \\
\hline Sn 106 & 105.9169 & $1.150 E+02$ & second & $1.15 E+02$ & $9.2622 \mathrm{E}+08$ & $1.0797 \mathrm{E}-09$ & & & \\
\hline Sn 107 & 106.9157 & $2.900 E+00$ & minute & $1.74 E+02$ & $6.0644 \mathrm{E}+08$ & $1.6490 \mathrm{E}-09$ & & & \\
\hline Sn 108 & 107.9120 & $1.030 E+01$ & minute & $6.18 E+02$ & $1.6917 E+08$ & $5.9113 E-09$ & & & \\
\hline Sn 109 & 108.9113 & $1.800 E+01$ & minute & $1.08 E+03$ & $9.5914 E+07$ & $1.0426 \mathrm{E}-08$ & & & \\
\hline Sn 110 & 109.9079 & $4.110 \mathrm{E}+00$ & hour & $1.48 E+04$ & $6.9375 \mathrm{E}+06$ & $1.4414 \mathrm{E}-07$ & & & \\
\hline Sn 111 & 110.9077 & $3.530 E+01$ & minute & $2.12 E+03$ & $4.8027 \mathrm{E}+07$ & $2.0821 E-08$ & & & \\
\hline $\operatorname{Sn~} 113$ & 112.9052 & $1.151 E+02$ & day & $9.94 E+06$ & $1.0049 E+04$ & $9.9515 \mathrm{E}-05$ & 0.002480 & & \\
\hline Sn 113m & 112.9052 & $2.140 E+01$ & minute & $1.28 E+03$ & $7.7821 E+07$ & $1.2850 \mathrm{E}-08$ & & & \\
\hline Sn $117 \mathrm{~m}$ & 116.9030 & $1.360 E+01$ & day & $1.18 E+06$ & $8.2129 \mathrm{E}+04$ & $1.2176 E-05$ & 0.001890 & & \\
\hline Sn $119 m$ & 118.9033 & $2.931 \mathrm{E}+02$ & day & $2.53 E+07$ & $3.7467 \mathrm{E}+03$ & $2.6690 \mathrm{E}-04$ & 0.000532 & & \\
\hline Sn 121 & 120.9042 & $1.128 E+00$ & day & $9.74 E+04$ & $9.5787 \mathrm{E}+05$ & $1.0440 \mathrm{E}-06$ & & & $1.280 \mathrm{E}-04$ \\
\hline Sn 121m & 120.9042 & $5.500 E+01$ & year & $1.74 \mathrm{E}+09$ & $5.3760 \mathrm{E}+01$ & $1.8601 \mathrm{E}-02$ & 0.000243 & & 7.107E-06 \\
\hline Sn 123 & 122.9057 & $1.292 \mathrm{E}+02$ & day & $1.12 E+07$ & $8.2230 \mathrm{E}+03$ & $1.2161 \mathrm{E}-04$ & 0.003140 & & 3.059E-05 \\
\hline Sn $123 \mathrm{~m}$ & 122.9057 & $4.006 E+01$ & minute & $2.40 E+03$ & $3.8189 \mathrm{E}+07$ & $2.6185 \mathrm{E}-08$ & & & $1.282 \mathrm{E}-04$ \\
\hline $\operatorname{Sn} 125$ & 124.9078 & $9.640 \mathrm{E}+00$ & day & $8.33 E+05$ & $1.0844 \mathrm{E}+05$ & $9.2215 \mathrm{E}-06$ & 0.006656 & & 8.197E-05 \\
\hline$S n 125 \mathrm{~m}$ & 124.9078 & $9.520 E+00$ & minute & $5.71 E+02$ & $1.5812 E+08$ & $6.3241 \mathrm{E}-09$ & & & $2.116 \mathrm{E}-04$ \\
\hline Sn 126 & 125.9077 & $1.000 \mathrm{E}+05$ & year & $3.16 \mathrm{E}+12$ & $2.8393 \mathrm{E}-02$ & $3.5220 \mathrm{E}+01$ & 0.001080 & & $5.493 \mathrm{E}-04$ \\
\hline$S$ & 126.9104 & $2.100 E+00$ & hour & $7.56 \mathrm{E}+03$ & $1.1759 \mathrm{E}+07$ & $8.5044 \mathrm{E}-08$ & & & $4.780 \mathrm{E}-04$ \\
\hline $\mathrm{Sn} 127 \mathrm{~m}$ & 126.9104 & $4.130 \mathrm{E}+00$ & minute & $2.48 \mathrm{E}+02$ & $3.5874 \mathrm{E}+08$ & $2.7875 \mathrm{E}-09$ & & & $7.094 \mathrm{E}-04$ \\
\hline Sn 128 & 127.9105 & $5.907 \mathrm{E}+01$ & minute & $3.54 \mathrm{E}+03$ & $2.4886 E+07$ & $4.0184 E-08$ & & & 3.350E-03 \\
\hline
\end{tabular}




\begin{tabular}{|c|c|c|c|c|c|c|c|c|c|}
\hline Isotope & $\begin{array}{c}\text { Atomic } \\
\text { Mass }\end{array}$ & Halflife & $\begin{array}{l}\text { Time } \\
\text { Units }\end{array}$ & $t_{1 / 2}, \sec$ & Curies/gram & Grams/ Curie & $\begin{array}{c}\text { Watts/Ci } \\
\text { (Ref.3) }\end{array}$ & $\begin{array}{c}\text { Individual }^{1} \\
\text { Fission } \\
\text { Product Yield } \\
\text { (Ref.4) }\end{array}$ & $\begin{array}{c}\text { Cumulative } \\
\text { Fission Product } \\
\text { Yield (Ref.4) } \\
\end{array}$ \\
\hline Sn $128 \mathrm{~m}$ & 127.9105 & $6.500 \mathrm{E}+00$ & second & $6.50 \mathrm{E}+00$ & $1.3569 \mathrm{E}+10$ & $7.3696 \mathrm{E}-11$ & & & \\
\hline Sn 129 & 128.9134 & $2.230 \mathrm{E}+00$ & minute & $1.34 E+02$ & $6.5407 \mathrm{E}+08$ & $1.5289 \mathrm{E}-09$ & & & $2.479 \mathrm{E}-03$ \\
\hline Sn $129 \mathrm{~m}$ & 128.9134 & $6.900 \mathrm{E}+00$ & minute & $4.14 E+02$ & $2.1139 E+08$ & 4.7307E-09 & & & 4.063E-03 \\
\hline $\operatorname{sn} 130$ & 129.9139 & $3.720 E+00$ & minute & $2.23 E+02$ & $3.8907 E+08$ & $2.5702 \mathrm{E}-09$ & & & $4.089 \mathrm{E}-03$ \\
\hline $\mathrm{sn} 130 \mathrm{~m}$ & 129.9139 & $1.700 \mathrm{E}+00$ & minute & $1.02 E+02$ & $8.5137 \mathrm{E}+08$ & $1.1746 \mathrm{E}-09$ & & & $7.390 \mathrm{E}-03$ \\
\hline $\operatorname{sn~} 131$ & 130.9169 & $5.600 E+01$ & second & $5.60 E+01$ & $1.5388 \mathrm{E}+09$ & $6.4984 E-10$ & & & $2.706 \mathrm{E}-03$ \\
\hline$S n 131 \mathrm{~m}$ & 130.9169 & $5.840 \mathrm{E}+01$ & second & $5.84 \mathrm{E}+01$ & $1.4756 \mathrm{E}+09$ & $6.7769 \mathrm{E}-10$ & & & $6.125 \mathrm{E}-03$ \\
\hline $\operatorname{sn} 132$ & 131.9177 & $3.970 E+01$ & second & $3.97 E+01$ & $2.1542 E+09$ & $4.6421 \mathrm{E}-10$ & & & $5.774 \mathrm{E}-03$ \\
\hline $\operatorname{sn} 133$ & 132.9238 & $1.440 \mathrm{E}+00$ & second & $1.44 \mathrm{E}+00$ & $5.8940 E+10$ & $1.6966 \mathrm{E}-11$ & & & $1.459 \mathrm{E}-03$ \\
\hline Sn 134 & 133.9285 & $1.040 E+00$ & second & $1.04 \mathrm{E}+00$ & $8.0997 \mathrm{E}+10$ & $1.2346 \mathrm{E}-11$ & & & $1.124 E-04$ \\
\hline Sb 108 & 107.9222 & $7.000 E+00$ & second & $7.00 E+00$ & $1.4934 E+10$ & $6.6963 \mathrm{E}-11$ & & & \\
\hline Sb 109 & 108.9181 & $1.700 E+01$ & second & $1.70 E+01$ & $6.0929 E+09$ & $1.6412 E-10$ & & & \\
\hline Sb 111 & 110.9132 & $7.500 E+01$ & second & $7.50 \mathrm{E}+01$ & $1.3562 E+09$ & $7.3734 \mathrm{E}-10$ & & & \\
\hline$S b 112$ & 111.9124 & $5.140 \mathrm{E}+01$ & second & $5.14 E+01$ & $1.9613 E+09$ & $5.0988 \mathrm{E}-10$ & & & \\
\hline$S b 113$ & 112.9094 & $6.670 E+00$ & minute & $4.00 \mathrm{E}+02$ & $2.4967 E+08$ & $4.0053 \mathrm{E}-09$ & & & \\
\hline Sb 114 & 113.9091 & $3.490 E+00$ & minute & $2.09 \mathrm{E}+02$ & $4.7298 \mathrm{E}+08$ & $2.1143 \mathrm{E}-09$ & & & \\
\hline$S b 115$ & 114.9066 & $3.210 E+01$ & minute & $1.93 \mathrm{E}+03$ & $5.0977 \mathrm{E}+07$ & $1.9617 \mathrm{E}-08$ & & & \\
\hline$S b 116$ & 115.9068 & $1.580 E+01$ & minute & $9.48 E+02$ & $1.0267 E+08$ & $9.7396 \mathrm{E}-09$ & & & \\
\hline $\mathrm{sb} 116 \mathrm{~m}$ & 115.9068 & $6.030 E+01$ & minute & $3.62 E+03$ & $2.6903 E+07$ & $3.7171 \mathrm{E}-08$ & & & \\
\hline Sb 117 & 116.9048 & $2.800 E+00$ & hour & $1.01 E+04$ & $9.5738 E+06$ & $1.0445 \mathrm{E}-07$ & & & \\
\hline Sb 118 & 117.9055 & $3.600 E+00$ & minute & $2.16 E+02$ & $4.4298 \mathrm{E}+08$ & $2.2574 E-09$ & & & \\
\hline Sb $118 \mathrm{~m}$ & 117.9055 & $5.000 E+00$ & hour & $1.80 E+04$ & $5.3158 E+06$ & $1.8812 E-07$ & & & \\
\hline Sb 119 & 118.9039 & $1.591 E+00$ & day, & $1.37 E+05$ & $6.9015 \mathrm{E}+05$ & $1.4490 \mathrm{E}-06$ & & & \\
\hline Sb 120 & 119.9051 & $1.589 \mathrm{E}+01$ & minute & $9.53 E+02$ & $9.8688 \mathrm{E}+07$ & $1.0133 \mathrm{E}-08$ & & & $3.752 \mathrm{E}-13$ \\
\hline Sb $120 \mathrm{~m}$ & 119.9051 & $5.760 E+00$ & day & $4.98 E+05$ & $1.8906 \mathrm{E}+05$ & $5.2893 \mathrm{E}-06$ & & & $5.326 \mathrm{E}-13$ \\
\hline Sb 122 & 121.9052 & $2.700 E+00$ & day & $2.33 E+05$ & $3.9671 E+05$ & $2.5207 \mathrm{E}-06$ & & & $2.909 \mathrm{E}-10$ \\
\hline$S b 122 m$ & 121.9052 & $4.210 E+00$ & minute & $2.53 E+02$ & $3.6637 \mathrm{E}+08$ & $2.7295 \mathrm{E}-09$ & & & $1.546 \mathrm{E}-10$ \\
\hline Sb 124 & 123.9059 & $6.020 E+01$ & day & $5.20 E+06$ & $1.7505 E+04$ & $5.7125 \mathrm{E}-05$ & 0.013290 & & $1.061 \mathrm{E}-07$ \\
\hline$S b$ 124m & 123.9059 & $1.550 E+00$ & minute & $9.30 \mathrm{E}+01$ & $9.7904 \mathrm{E}+08$ & $1.0214 \mathrm{E}-09$ & & & $9.068 \mathrm{E}-08$ \\
\hline $\mathrm{Sb} 124 \mathrm{~m} 2$ & 123.9059 & $2.020 E+01$ & minute & $1.21 E+03$ & $7.5125 \mathrm{E}+07$ & $1.3311 \mathrm{E}-08$ & & & \\
\hline Sb 125 & 124.9052 & $2.758 \mathrm{E}+00$ & year & $8.70 \mathrm{E}+07$ & $1.0377 \mathrm{E}+03$ & $9.6371 \mathrm{E}-04$ & 0.003370 & & $2.938 \mathrm{E}-04$ \\
\hline Sb 126 & 125.9072 & $1.246 \mathrm{E}+01$ & day & $1.08 \mathrm{E}+06$ & $8.3233 E+04$ & $1.2015 \mathrm{E}-05$ & 0.018390 & & $7.807 \mathrm{E}-05$ \\
\hline $\mathrm{Sb} 126 \mathrm{~m}$ & 125.9072 & $1.915 E+01$ & minute & $1.15 E+03$ & $7.7984 E+07$ & $1.2823 \mathrm{E}-08$ & 0.012920 & & $5.509 \mathrm{E}-04$ \\
\hline Sb 127 & 126.9069 & $3.850 \mathrm{E}+00$ & day & $3.33 E+05$ & $2.6725 E+05$ & $3.7418 \mathrm{E}-06$ & & & $1.246 \mathrm{E}-03$ \\
\hline Sb 128 & 127.9092 & $9.010 E+00$ & hour & $3.24 E+04$ & $2.7192 E+06$ & $3.6775 \mathrm{E}-07$ & & & $1.896 \mathrm{E}-04$ \\
\hline
\end{tabular}




\begin{tabular}{|c|c|c|c|c|c|c|c|c|c|}
\hline Isotope & $\begin{array}{c}\text { Atomic } \\
\text { Mass }\end{array}$ & Halflife & $\begin{array}{l}\text { Time } \\
\text { Units }\end{array}$ & $t_{1 / 2}, \sec$ & Curies/ gram & Grams/ Curie & $\begin{array}{c}\text { Watts/Ci } \\
\text { (Ref.3) }\end{array}$ & $\begin{array}{c}\text { Indlvidual }^{1} \\
\text { Fission } \\
\text { Product Yield } \\
\text { (Ref.4) }\end{array}$ & $\begin{array}{l}\text { Cumulative } \\
\text { Fission Product } \\
\text { Yield (Ref.4) }\end{array}$ \\
\hline $\mathrm{Sb} 128 \mathrm{~m}$ & 127.9092 & $1.040 E+01$ & minute & $6.24 E+02$ & $1.4135 E+08$ & 7.0747E-09 & & & $3.461 \mathrm{E}-03$ \\
\hline Sb 129 & 128.9092 & $4.400 E+00$ & hour & $1.58 \mathrm{E}+04$ & $5.5251 \mathrm{E}+06$ & $1.8099 \mathrm{E}-07$ & & & $7.128 \mathrm{E}-03$ \\
\hline $\mathrm{Sb} 129 \mathrm{~m}$ & 128.9092 & $1.770 E+01$ & minute & $1.06 \mathrm{E}+03$ & $8.2408 E+07$ & $1.2135 \mathrm{E}-08$ & & & $3.238 E-04$ \\
\hline $\mathrm{Sb} 130$ & 129.9115 & $3.950 E+01$ & minute & $2.37 E+03$ & $3.6642 E+07$ & $2.7291 \mathrm{E}-08$ & & & $7.432 \mathrm{E}-03$ \\
\hline $\mathrm{Sb} 130 \mathrm{~m}$ & 129.9115 & $6.300 E+00$ & minute & $3.78 E+02$ & $2.2974 E+08$ & 4.3527E-09 & & & $9.853 \mathrm{E}-03$ \\
\hline Sb 131 & 130.9119 & $2.303 E+01$ & minute & $1.38 \mathrm{E}+03$ & $6.2367 E+07$ & $1.6034 \mathrm{E}-08$ & & & $2.581 \mathrm{E}-02$ \\
\hline Sb 132 & 131.9144 & $2.790 E+00$ & minute & $1.67 E+02$ & $5.1089 E+08$ & $1.9574 E-09$ & & & $1.852 \mathrm{E}-02$ \\
\hline $\mathrm{Sb} 132 \mathrm{~m}$ & 131.9144 & $4.100 E+00$ & minute & $2.46 E+02$ & $3.4766 E+08$ & $2.8764 E-09$ & & & $9.393 \mathrm{E}-03$ \\
\hline $\mathrm{Sb} 133$ & 132.9152 & $2.500 E+00$ & minute & $1.50 E+02$ & $5.6586 E+08$ & $1.7672 E-09$ & & & $2.373 E-02$ \\
\hline Sb 134 & 133.9206 & $7.800 \mathrm{E}-01$ & second & $7.80 \mathrm{E}-01$ & $1.0800 \mathrm{E}+11$ & $9.2590 \mathrm{E}-12$ & & & $1.438 \mathrm{E}-03$ \\
\hline $\mathrm{Sb} 134 \mathrm{~m}$ & 133.9206 & $1.022 E+01$ & second & $1.02 E+01$ & $8.2429 E+09$ & $1.2132 E-10$ & & & $5.656 \mathrm{E}-03$ \\
\hline $\mathrm{Sb} 135$ & 134.9252 & $1.710 \mathrm{E}+00$ & second & $1.71 \mathrm{E}+00$ & $4.8898 E+10$ & $2.0451 \mathrm{E}-11$ & & & 1.463E-03 \\
\hline Sb 136 & 135.9307 & $8.200 E-01$ & second & $8.20 \mathrm{E}-01$ & $1.0122 E+11$ & $9.8800 \mathrm{E}-12$ & & & 1.577E-04 \\
\hline Te 107 & 106.9157 & $3.100 \mathrm{E}-03$ & second & $3.10 \mathrm{E}-03$ & $3.4039 \mathrm{E}+13$ & $2.9378 E-14$ & & & \\
\hline $\mathrm{Te} 108$ & 107.9120 & $2.100 \mathrm{E}+00$ & second & $2.10 \mathrm{E}+00$ & $4.9784 E+10$ & $2.0087 E-11$ & & & \\
\hline Te 109 & 108.9275 & $4.600 \mathrm{E}+00$ & second & $4.60 \mathrm{E}+00$ & $2.2515 E+10$ & $4.4414 E-11$ & & & \\
\hline Te 110 & 109.9224 & $1.860 \mathrm{E}+01$ & second & $1.86 \mathrm{E}+01$ & $5.5179 E+09$ & $1.8123 E-10$ & & & \\
\hline Te 111 & 110.9211 & $1.930 \mathrm{E}+01$ & second & $1.93 E+01$ & $5.2699 E+09$ & $1.8976 E-10$ & & & \\
\hline Te 112 & 111.9171 & $2.000 E+00$ & minute & $1.20 \mathrm{E}+02$ & $8.4004 E+08$ & $1.1904 E-09$ & & & \\
\hline Te 113 & 112.9159 & $1.700 \mathrm{E}+00$ & minute & $1.02 E+02$ & $9.7954 \mathrm{E}+08$ & $1.0209 E-09$ & & & \\
\hline Te 114 & 113.9121 & $1.520 \mathrm{E}+01$ & minute & $9.12 \mathrm{E}+02$ & $1.0860 \mathrm{E}+08$ & $9.2085 E-09$ & & & \\
\hline Te 115 & 114.9116 & $5.800 \mathrm{E}+00$ & minute & $3.48 \mathrm{E}+02$ & $2.8212 E+08$ & 3.5446E-09 & & & \\
\hline $\mathrm{Te} 115 \mathrm{~m}$ & 114.9116 & $6.700 \mathrm{E}+00$ & minute & $4.02 E+02$ & $2.4422 E+08$ & $4.0946 E-09$ & & & \\
\hline Te 116 & 115.9084 & $2.490 \mathrm{E}+00$ & hour & $8.96 E+03$ & $1.0858 E+07$ & $9.2096 E-08$ & & & \\
\hline Te 117 & 116.9086 & $1.033 \mathrm{E}+00$ & hour & $3.72 E+03$ & $2.5942 \mathrm{E}+07$ & $3.8548 E-08$ & & & \\
\hline Te 118 & 117.9058 & $6.000 \mathrm{E}+00$ & day & $5.18 E+05$ & $1.8458 E+05$ & $5.4178 \mathrm{E}-06$ & & & \\
\hline Te 119 & 118.9064 & $1.603 \mathrm{E}+01$ & hour & $5.77 E+04$ & $1.6441 E+06$ & $6.0823 \mathrm{E}-07$ & & & \\
\hline $\mathrm{Te} 119 \mathrm{~m}$ & 118.9064 & $4.700 E+00$ & day & $4.06 E+05$ & $2.3365 E+05$ & 4.2800E-06 & & & \\
\hline Te 121 & 120.9049 & $1.678 \mathrm{E}+01$ & day & $1.45 E+06$ & $6.4362 E+04$ & 1.5537E-05 & & & \\
\hline $\mathrm{Te} 121 \mathrm{~m}$ & 120.9049 & $1.540 E+02$ & day & $1.33 E+07$ & $7.0129 E+03$ & $1.4259 \mathrm{E}-04$ & & & \\
\hline Te 123 & 122.9043 & $1.300 \mathrm{E}+13$ & year & $4.10 E+20$ & $2.2374 \mathrm{E}-10$ & $4.4694 \mathrm{E}+09$ & & & $1.350 \mathrm{E}-12$ \\
\hline $\mathrm{Te} 123 \mathrm{~m}$ & 122.9043 & 1.197E+02 & day & $1.03 E+07$ & $8.8757 E+03$ & 1.1267E-04 & 0.001482 & & $1.034 E-12$ \\
\hline $\mathrm{Te} 125 \mathrm{~m}$ & 124.9044 & $5.740 E+01$ & day & $4.96 E+06$ & $1.8213 E+04$ & 5.4907E-05 & 0.000869 & & $6.404 \mathrm{E}-05$ \\
\hline Te 127 & 126.9052 & $9.350 E+00$ & hour & $3.37 E+04$ & $2.6411 E+06$ & 3.7863E-07 & 0.001360 & & $1.242 E-03$ \\
\hline $\mathrm{Te} 127 \mathrm{~m}$ & 126.9052 & $1.090 E+02$ & day & $9.42 E+06$ & $9.4397 E+03$ & $1.0594 \mathrm{E}-04$ & 0.000552 & & 1.733E-04 \\
\hline
\end{tabular}

WSRC-TR-99-00344

October, 1999

page 30 


\begin{tabular}{|c|c|c|c|c|c|c|c|c|c|}
\hline Isotope & $\begin{array}{l}\text { Atomic } \\
\text { Mass }\end{array}$ & Halflife & $\begin{array}{l}\text { Time } \\
\text { Units }\end{array}$ & $\mathbf{t}_{1 / 2}, \mathrm{sec}$ & Curies/gram & Grams/ Curie & $\begin{array}{c}\text { Watts/Ci } \\
\text { (Ref.3) }\end{array}$ & $\begin{array}{c}\text { Individual }^{1} \\
\text { Fission } \\
\text { Product Yield } \\
\text { (Ref.4) } \\
\end{array}$ & $\begin{array}{c}\text { Cumulative } \\
\text { Fission Product } \\
\text { Yield (Ref.4) }\end{array}$ \\
\hline Te 128 & 127.9045 & $8.000 E+24$ & year & $2.52 E+32$ & $3.4937 \mathrm{E}-22$ & $2.8623 \mathrm{E}+21$ & & & $3.528 \mathrm{E}-03$ \\
\hline Te 129 & 128.9066 & $6.960 \mathrm{E}+01$ & minute & $4.18 \mathrm{E}+03$ & $2.0958 \mathrm{E}+07$ & $4.7715 \mathrm{E}-08$ & 0.003580 & & $6.926 \mathrm{E}-03$ \\
\hline Te $129 \mathrm{~m}$ & 128.9066 & $3.360 E+01$ & day & $2.90 E+06$ & $3.0147 E+04$ & $3.3170 \mathrm{E}-05$ & 0.001800 & & $7.199 \mathrm{E}-04$ \\
\hline $\mathrm{Te} 130$ & 129.9062 & $2.700 E+21$ & year & $8.52 E+28$ & $1.0192 \mathrm{E}-18$ & $9.8114 \mathrm{E}+17$ & & & $1.785 \mathrm{E}-02$ \\
\hline Te 131 & 130.9085 & $2.500 E+01$ & minute & $1.50 E+03$ & $5.7454 \mathrm{E}+07$ & $1.7405 \mathrm{E}-08$ & & & $2.483 \mathrm{E}-02$ \\
\hline Te $131 \mathrm{~m}$ & 130.9085 & $3.000 E+01$ & hour & $1.08 E+05$ & $7.9797 E+05$ & $1.2532 \mathrm{E}-06$ & & & $5.110 \mathrm{E}-03$ \\
\hline Te 132 & 131.9085 & $3.204 \mathrm{E}+00$ & day & $2.77 \mathrm{E}+05$ & $3.0896 E+05$ & $3.2367 \mathrm{E}-06$ & & & $4.283 \mathrm{E}-02$ \\
\hline$T e 133$ & 132.9109 & $1.250 E+01$ & minute & $7.50 E+02$ & $1.1318 \mathrm{E}+08$ & $8.8358 \mathrm{E}-09$ & & & $3.663 \mathrm{E}-02$ \\
\hline Te $133 \mathrm{~m}$ & 132.9109 & $5.540 E+01$ & minute & $3.32 E+03$ & $2.5536 \mathrm{E}+07$ & $3.9160 \mathrm{E}-08$ & & & $3.359 \mathrm{E}-02$ \\
\hline Te 134 & 133.9115 & $4.180 E+01$ & minute & $2.51 E+03$ & $3.3592 \mathrm{E}+07$ & $2.9769 \mathrm{E}-08$ & & & $6.912 \mathrm{E}-02$ \\
\hline Te 135 & 134.9165 & $1.900 E+01$ & second & $1.90 E+01$ & $4.4011 E+09$ & $2.2722 \mathrm{E}-10$ & & & $3.243 \mathrm{E}-02$ \\
\hline Te 136 & 135.9201 & $1.750 E+01$ & second & $1.75 E+01$ & $4.7430 E+09$ & $2.1084 \mathrm{E}-10$ & & & $1.533 \mathrm{E}-02$ \\
\hline Te 137 & 136.9253 & $2.490 E+00$ & second & $2.49 \mathrm{E}+00$ & $3.3090 \mathrm{E}+10$ & $3.0221 \mathrm{E}-11$ & & & $4.256 \mathrm{E}-03$ \\
\hline Te 138 & 137.9224 & $1.400 E+00$ & second & $1.40 E+00$ & $5.8427 E+10$ & & & & $6.746 \mathrm{E}-04$ \\
\hline 1108 & 107.9433 & $5.000 \mathrm{E}-02$ & second & $5.00 \mathrm{E}-02$ & $2.0903 E+12$ & $4.7840 \mathrm{E}-13$ & & & \\
\hline 1109 & 108.9382 & $1.100 E-04$ & second & $1.10 \mathrm{E}-04$ & $9.4146 E+14$ & & & & \\
\hline 1110 & 109.9352 & $6.500 \mathrm{E}-01$ & second & $6.50 \mathrm{E}-01$ & $1.5788 \mathrm{E}+11$ & $6.3339 \mathrm{E}-12$ & & & \\
\hline 1111 & 110.9303 & $2.500 E+00$ & second & $2.50 \mathrm{E}+00$ & $4.0681 E+10$ & $2.4582 \mathrm{E}-11$ & & & \\
\hline 1112 & 111.9280 & $3.420 \mathrm{E}+00$ & second & $3.42 E+00$ & $2.9472 E+10$ & $3.3930 \mathrm{E}-11$ & & & \\
\hline 1113 & 112.9236 & $6.600 \mathrm{E}+00$ & second & $6.60 \mathrm{E}+00$ & $1.5137 \mathrm{E}+10$ & $6.6062 \mathrm{E}-11$ & & & \\
\hline 1114 & 113.9219 & $2.100 E+00$ & second & $2.10 E+00$ & $4.7157 E+10$ & $2.1206 \mathrm{E}-11$ & & & \\
\hline 1115 & 114.9179 & $1.300 \mathrm{E}+00$ & minute & $7.80 E+01$ & $1.2586 \mathrm{E}+09$ & $7.9452 \mathrm{E}-10$ & & & \\
\hline 1116 & 115.9167 & $2.910 E+00$ & second & $2.91 \mathrm{E}+00$ & $3.3445 \mathrm{E}+10$ & $2.9899 \mathrm{E}-11$ & & & \\
\hline 1117 & 116.9136 & $2.220 E+00$ & minute & $1.33 E+02$ & $7.2445 E+08$ & $1.3804 \mathrm{E}-09$ & & & \\
\hline 1118 & 117.9134 & $1.370 \mathrm{E}+01$ & minute & $8.22 E+02$ & $1.1640 \mathrm{E}+08$ & $8.5913 E-09$ & & & \\
\hline $1118 \mathrm{~m}$ & 117.9134 & $8.500 E+00$ & minute & $5.10 \mathrm{E}+02$ & $1.8760 \mathrm{E}+08$ & $5.3304 \mathrm{E}-09$ & & & \\
\hline 1119 & 118.9102 & $1.910 \mathrm{E}+01$ & minute & $1.15 \mathrm{E}+03$ & $8.2789 \mathrm{E}+07$ & $1.2079 \mathrm{E}-08$ & & & \\
\hline 1120 & 119.9100 & $1.350 E+00$ & hour & $4.86 \mathrm{E}+03$ & $1.9359 \mathrm{E}+07$ & $5: 1655 E-08$ & & & \\
\hline $1120 \mathrm{~m}$ & 119.9100 & $5.300 \mathrm{E}+01$ & minute & $3.18 \mathrm{E}+03$ & $2.9587 \mathrm{E}+07$ & $3.3799 \mathrm{E}-08$ & & & \\
\hline 1121 & 120.9074 & $2.120 E+00$ & hour & $7.63 E+03$ & $1.2226 \mathrm{E}+07$ & $8.1793 \mathrm{E}-08$ & & & \\
\hline 1122 & 121.9076 & $3.630 \mathrm{E}+00$ & minute & $2.18 \mathrm{E}+02$ & $4.2490 E+08$ & $2.3535 \mathrm{E}-09$ & & & $3.497 \mathrm{E}-21$ \\
\hline 1123 & 122.9056 & $1.327 E+01$ & hour & $4.78 E+04$ & $1.9215 E+06$ & $5.2044 \mathrm{E}-07$ & 0.001188 & & $9.017 \mathrm{E}-19$ \\
\hline 1124 & 123.9062 & $4.180 E+00$ & day & $3.61 \mathrm{E}+05$ & $2.5211 \mathrm{E}+05$ & $3.9665 \mathrm{E}-06$ & & & $1.187 \mathrm{E}-17$ \\
\hline 1125 & 124.9046 & $5.941 \mathrm{E}+01$ & day & $5.13 E+06$ & 1.7597E+04 & $5.6828 \mathrm{E}-05$ & 0.000357 & & $6.090 \mathrm{E}-16$ \\
\hline 1126 & 125.9056 & $1.311 \mathrm{E}+01$ & day & $1.13 E+06$ & $7.9107 \mathrm{E}+04$ & $1.2641 \mathrm{E}-05$ & & & $2.809 \mathrm{E}-12$ \\
\hline
\end{tabular}

WSRC-TR-99-00344

October, 1999

page 31 


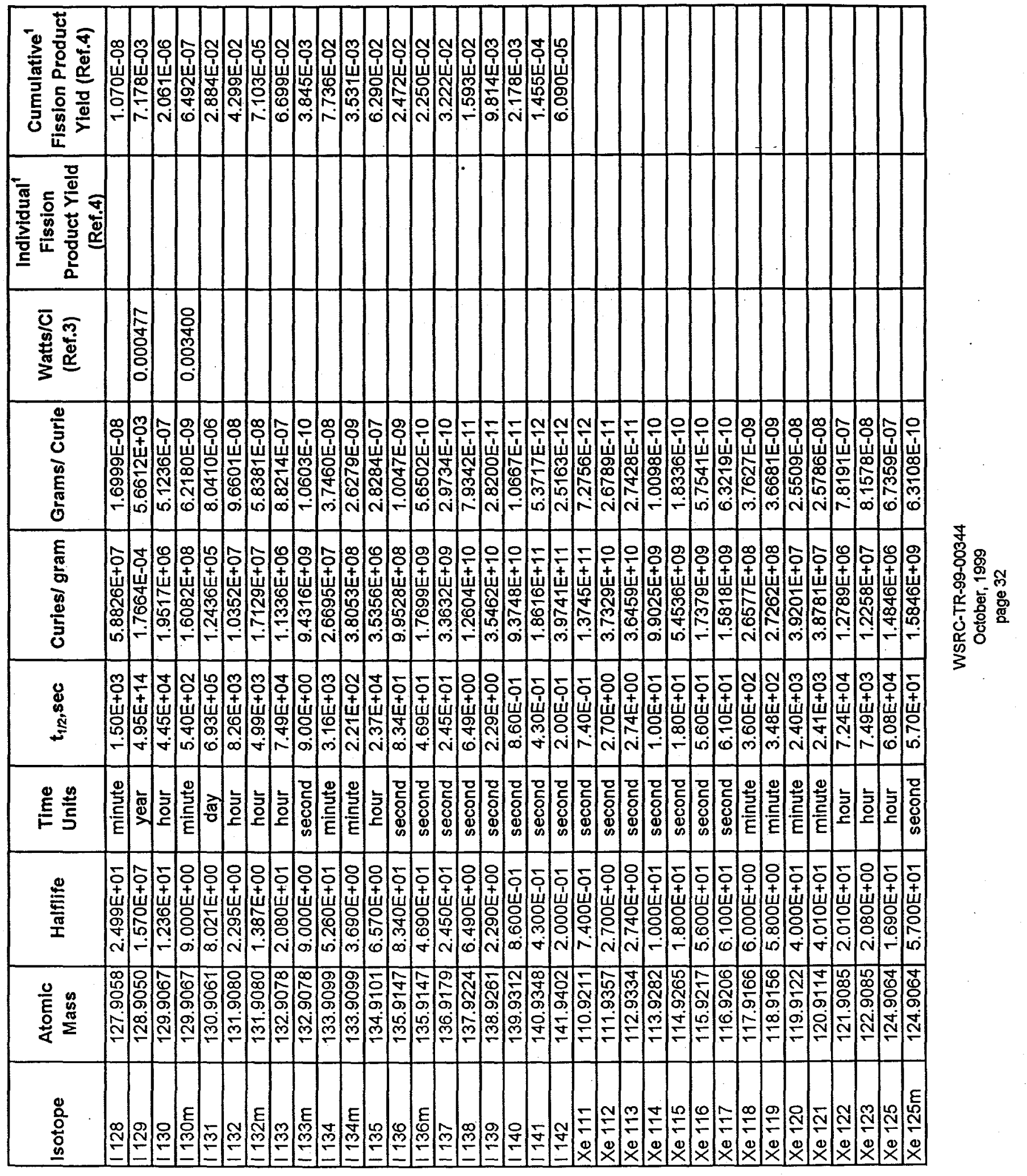




\begin{tabular}{|c|c|c|c|c|c|c|c|c|c|}
\hline Isotope & $\begin{array}{l}\text { Atomic } \\
\text { Mass }\end{array}$ & Halflife & $\begin{array}{l}\text { Time } \\
\text { Units }\end{array}$ & $t_{1 / 2}, \sec$ & Curies/gram & Grams/ Curie & $\begin{array}{c}\text { Watts/Ci } \\
\text { (Ref.3) }\end{array}$ & $\begin{array}{c}\text { Individual }^{4} \\
\text { Fission } \\
\text { Product Yield } \\
\text { (Ref.4) }\end{array}$ & $\begin{array}{c}\text { Cumulative } \\
\text { Fission Product } \\
\text { Yield (Ref.4) }\end{array}$ \\
\hline Xe 127 & 126.9052 & $3.640 E+01$ & day & $3.14 E+06$ & $2.8267 E+04$ & $3.5377 \mathrm{E}-05$ & & & \\
\hline Xe 127m & 126.9052 & $6.920 E+01$ & second & $6.92 \mathrm{E}+01$ & $1.2847 \mathrm{E}+09$ & $7.7841 \mathrm{E}-10$ & & & $1.805 \mathrm{E}-17$ \\
\hline Xe 129m & 128.9048 & $8.880 E+00$ & day & $7.67 \mathrm{E}+05$ & $1.1407 E+05$ & $8.7664 E-06$ & & & $3.477 \mathrm{E}-12$ \\
\hline$X e 131 \mathrm{~m}$ & 130.9051 & $1.184 E+01$ & day & $1.02 E+06$ & $8.4247 \mathrm{E}+04$ & $1.1870 \mathrm{E}-05$ & & & $3.173 \mathrm{E}-04$ \\
\hline Xe 132m & 131.9042 & $8.390 \mathrm{E}-03$ & second & $8.39 \mathrm{E}-03$ & $1.0194 E+13$ & $9.8094 \mathrm{E}-14$ & & & \\
\hline Xe 133 & 132.9059 & $5.243 E+00$ & day & $4.53 E+05$ & $1.8739 \mathrm{E}+05$ & $5.3366 \mathrm{E}-06$ & 0.001080 & & $6.702 E-02$ \\
\hline$X e 133 m$ & 132.9059 & $2.190 E+00$ & day & $1.89 E+05$ & $4.4862 E+05$ & $2.2291 \mathrm{E}-06$ & & & $1.949 \mathrm{E}-03$ \\
\hline Xe 134m & 133.9099 & $2.900 \mathrm{E}-01$ & second & $2.90 E-01$ & $2.9051 E+11$ & $3.4422 E-12$ & & & $6.564 E-04$ \\
\hline Xe 135 & 134.9072 & $9.140 \mathrm{E}+00$ & hour & $3.29 E+04$ & $2.5415 \mathrm{E}+06$ & $3.9347 \mathrm{E}-07$ & & & $6.532 \mathrm{E}-02$ \\
\hline $\mathrm{Xe} 135 \mathrm{~m}$ & 134.9072 & $1.529 \mathrm{E}+01$ & minute & $9.17 E+02$ & $9.1155 \mathrm{E}+07$ & $1.0970 \mathrm{E}-08$ & & & $1.213 E-02$ \\
\hline $\mathrm{Xe} 136$ & 135.9072 & $9.300 E+19$ & year & $2.93 E+27$ & $2.8284 \mathrm{E}-17$ & $3.5356 E+16$ & & & $6.306 \mathrm{E}-02$ \\
\hline Xe 137 & 136.9116 & $3.818 E+00$ & minute & $2.29 E+02$ & $3.5971 E+08$ & $2.7800 \mathrm{E}-09$ & & & $6.111 \mathrm{E}-02$ \\
\hline $\mathrm{Xe} 138$ & 137.9140 & $1.408 E+01$ & minute & $8.46 E+02$ & $9.6831 E+07$ & $1.0327 E-08$ & & & $6.373 E-02$ \\
\hline Xe 139 & 138.9188 & $3.968 E+01$ & second & $3.97 \mathrm{E}+01$ & $2.0467 \mathrm{E}+09$ & $4.8860 E-10$ & & & $5.141 \mathrm{E}-02$ \\
\hline $\mathrm{Xe} 140$ & 139.9216 & $1.360 E+01$ & second & $1.36 E+01$ & $5.9286 \mathrm{E}+09$ & $1.6867 \mathrm{E}-10$ & & & $3.768 \mathrm{E}-02$ \\
\hline $\mathrm{Xe} 141$ & 140.9266 & $1.730 E+00$ & second & $1.73 E+00$ & $4.6274 E+10$ & $2.1610 \mathrm{E}-11$ & & & $1.319 \mathrm{E}-02$ \\
\hline Xe 142 & 141.9297 & $1.220 E+00$ & second & $1.22 \mathrm{E}+00$ & $6.5154 E+10$ & $1.5348 \mathrm{E}-11$ & & & $4.454 \mathrm{E}-03$ \\
\hline Xe 143 & 142.9349 & $3.000 \mathrm{E}-01$ & second & $3.00 \mathrm{E}-01$ & $2.6310 E+11$ & $3.8009 \mathrm{E}-12$ & & & $1.555 E-04$ \\
\hline Xe143m & 142.9349 & $9.600 \mathrm{E}-01$ & second & $9.60 \mathrm{E}-01$ & $8.2218 \mathrm{E}+10$ & $1.2163 \mathrm{E}-11$ & & & $4.668 \mathrm{E}-04$ \\
\hline $\mathrm{Xe} 144$ & 143.9382 & $1.150 \mathrm{E}+00$ & second & $1.15 \mathrm{E}+00$ & $6.8156 E+10$ & $1.4672 \mathrm{E}-11$ & & & $6.257 \mathrm{E}-05$ \\
\hline$X e 145$ & 144.9437 & $9.000 \mathrm{E}-01$ & second & $9.00 \mathrm{E}-01$ & $8.6484 E+10$ & $1.1563 \mathrm{E}-11$ & & & $7.918 \mathrm{E}-07$ \\
\hline Cs 114 & 113.9414 & $5.700 \mathrm{E}-01$ & second & $5.70 \mathrm{E}-01$ & $1.7371 \mathrm{E}+11$ & $5.7568 \mathrm{E}-12$ & & & \\
\hline Cs 115 & 114.9359 & $1.400 E+00$ & second & $1.40 \mathrm{E}+00$ & $7.0112 E+10$ & $1.4263 \mathrm{E}-11$ & & & \\
\hline Cs116 & 115.9329 & $3.840 \mathrm{E}+00$ & second & $3.84 E+00$ & $2.5342 \mathrm{E}+10$ & $3.9460 \mathrm{E}-11$ & & & \\
\hline Cs $116 \mathrm{~m}$ & 115.9329 & $7.000 \mathrm{E}-01$ & second & $7.00 \mathrm{E}-01$ & $1.3902 E+11$ & $7.1933 \mathrm{E}-12$ & & & \\
\hline Cs 117 & 116.9286 & $8.400 E+00$ & second & $8.40 E+00$ & $1.1486 E+10$ & $8.7061 \mathrm{E}-11$ & & & \\
\hline Cs $117 \mathrm{~m}$ & 116.9286 & $6.500 E+00$ & second & $6.50 \mathrm{E}+00$ & $1.4844 E+10$ & $6.7369 \mathrm{E}-11$ & & & \\
\hline Cs 118 & 117.9266 & $1.400 \mathrm{E}+01$ & second & $1.40 \mathrm{E}+01$ & $6.8334 E+09$ & $1.4634 \mathrm{E}-10$ & & & \\
\hline Cs $118 \mathrm{~m}$ & 117.9266 & $1.700 E+01$ & second & $1.70 \mathrm{E}+01$ & $5.6275 E+09$ & $1.7770 \mathrm{E}-10$ & & & \\
\hline Cs 119 & 118.9224 & $4.300 E+01$ & second & $4.30 E+01$ & $2.2062 E+09$ & $4.5327 \mathrm{E}-10$ & & & \\
\hline Cs $119 \mathrm{~m}$ & 118.9224 & $3.040 E+01$ & second & $3.04 E+01$ & $3.1206 E+09$ & $3.2045 \mathrm{E}-10$ & & & \\
\hline Cs 120 & 119.9207 & $6.400 E+01$ & second & $6.40 E+01$ & $1.4699 E+09$ & $6.8030 \mathrm{E}-10$ & & & \\
\hline Cs $120 \mathrm{~m}$ & 119.9207 & $5.700 E+01$ & second & $5.70 E+01$ & $1.6505 E+09$ & $6.0589 \mathrm{E}-10$ & & & \\
\hline Cs 121 & 120.9172 & $1.280 \mathrm{E}+02$ & second & $1.28 \mathrm{E}+02$ & $7.2892 \mathrm{E}+08$ & $1.3719 \mathrm{E}-09$ & & & \\
\hline Cs121m & 120.9172 & $1.220 \mathrm{E}+02$ & second & $1.22 \mathrm{E}+02$ & $7.6477 \mathrm{E}+08$ & $1.3076 \mathrm{E}-09$ & & & \\
\hline
\end{tabular}


Radionuclide Specific Data

\begin{tabular}{|c|c|c|c|c|c|c|c|c|c|}
\hline Isotope & $\begin{array}{c}\text { Atomic } \\
\text { Mass }\end{array}$ & Halflife & $\begin{array}{l}\text { Time } \\
\text { Units }\end{array}$ & $t_{1 / 2}, \sec$ & Curies/gram & Grams/ Curie & $\begin{array}{c}\text { Watts/Ci } \\
\text { (Ref.3) }\end{array}$ & $\begin{array}{c}\text { Individual }^{4} \\
\text { Fission } \\
\text { Product Yield } \\
\text { (Ref.4) }\end{array}$ & $\begin{array}{l}\text { Cumulative }^{1} \\
\text { Fission Product } \\
\text { Yield (Ref.4) }\end{array}$ \\
\hline Cs 122 & 121.9161 & $2.100 \mathrm{E}+01$ & second & $2.10 E+01$ & $4.4065 E+09$ & $2.2694 \mathrm{E}-10$ & & & \\
\hline Cs $122 m$ & 121.9161 & $4.500 E+00$ & minute & $2.70 \mathrm{E}+02$ & $3.4273 E+08$ & 2.9178E-09 & & & \\
\hline Cs $122 \mathrm{~m} 2$ & 121.9161 & $3.600 E-01$ & second & $3.60 \mathrm{E}-01$ & $2.5705 E+11$ & $3.8903 E-12$ & & & \\
\hline Cs 123 & 122.9130 & $5.940 E+00$ & minute & $3.56 E+02$ & $2.5754 \mathrm{E}+08$ & $3.8829 E-09$ & & & \\
\hline Cs $123 \mathrm{~m}$ & 122.9130 & $1.640 E+00$ & second & $1.64 E+00$ & $5.5967 \mathrm{E}+10$ & $1.7868 \mathrm{E}-11$ & & & \\
\hline Cs 124 & 123.9122 & $3.080 E+01$ & second & $3.08 \mathrm{E}+01$ & $2.9560 \mathrm{E}+09$ & $3.3829 \mathrm{E}-10$ & & & \\
\hline Cs $124 \mathrm{~m}$ & 123.9122 & $6.300 E+00$ & second & $6.30 \mathrm{E}+00$ & $1.4452 E+10$ & $6.9196 \mathrm{E}-11$ & & & \\
\hline Cs 125 & 124.9097 & $4.500 E+01$ & minute & $2.70 \mathrm{E}+03$ & $3.3452 E+07$ & $2.9894 E-08$ & & & \\
\hline Cs 126 & 125.9094 & $1.640 E+00$ & minute & $9.84 E+01$ & $9.1059 E+08$ & $1.0982 E-09$ & & & \\
\hline Cs 127 & 126.9074 & $6.250 \mathrm{E}+00$ & hour & $2.25 E+04$ & $3.9510 \mathrm{E}+06$ & 2.5310 E-07 & & & \\
\hline Cs 128 & 127.9077 & $3.660 \mathrm{E}+00$ & minute & $2.20 E+02$ & $4.0165 \mathrm{E}+08$ & 2.4897E-09 & & & \\
\hline Cs 129 & 128.9061 & $3.206 E+01$ & hour & $1.15 \mathrm{E}+05$ & $7.5829 E+05$ & $1.3188 E-06$ & & & \\
\hline Cs 130 & 129.9067 & $2.921 E+01$ & minute & $1.75 E+03$ & $4.9552 E+07$ & $2.0181 E-08$ & & & \\
\hline Cs $130 \mathrm{~m}$ & 129.9067 & $3.460 \mathrm{E}+00$ & minute & $2.08 E+02$ & $4.1833 E+08$ & $2.3905 \mathrm{E}-09$ & & & \\
\hline Cs 131 & 130.9055 & $9.689 E+00$ & day & $8.37 \mathrm{E}+05$ & $1.0295 E+05$ & 9.7135E-06 & & & \\
\hline Cs 132 & 131.9064 & $6.479 \mathrm{E}+00$ & day & $5.60 \mathrm{E}+05$ & $1.5279 E+05$ & $6.5450 \mathrm{E}-06$ & & & $7.408 \mathrm{E}-10$ \\
\hline Cs 134 & 133.9067 & $2.062 E+00$ & year & $6.51 \mathrm{E}+07$ & $1.2947 \mathrm{E}+03$ & $7.7238 \mathrm{E}-04$ & 0.010190 & & 1.270E-07 \\
\hline Cs $134 \mathrm{~m}$ & 133.9067 & $2.910 \mathrm{E}+00$ & hour & $1.05 E+04$ & $8.0423 E+06$ & $1.2434 \mathrm{E}-07$ & & & $5.387 E-08$ \\
\hline Cs 135 & 134.9060 & $2.300 \mathrm{E}+06$ & year & $7.26 E+13$ & $1.1521 \mathrm{E}-03$ & $8.6796 E+02$ & 0.000330 & & $6.534 \mathrm{E}-02$ \\
\hline Cs $135 \mathrm{~m}$ & 134.9060 & $5.300 \mathrm{E}+01$ & minute & $3.18 \mathrm{E}+03$ & $2.6298 \mathrm{E}+07$ & $3.8026 E-08$ & & & $7.899 \mathrm{E}-06$ \\
\hline Cs 136 & 135.9072 & $1.316 E+01$ & day & $1.14 \mathrm{E}+06$ & $7.3007 E+04$ & 1.3697E-05 & & & $5.472 E-05$ \\
\hline Cs 136m & 135.9072 & $1.900 \mathrm{E}+01$ & second & $1.90 \mathrm{E}+01$ & $4.3690 E+09$ & $2.2889 E-10$ & & & 1.637E-05 \\
\hline Cs 137 & 136.9071 & $3.007 E+01$ & year & $9.49 E+08$ & $8.6836 E+01$ & $1.1516 E-02$ & 0.001010 & & $6.269 \mathrm{E}-02$ \\
\hline Cs 138 & 137.9110 & $3.341 \mathrm{E}+01$ & minute & $2.00 \mathrm{E}+03$ & $4.0808 \mathrm{E}+07$ & $2.4505 E-08$ & & & $6.646 \mathrm{E}-02$ \\
\hline Cs $138 \mathrm{~m}$ & 137.9110 & $2.910 E+00$ & minute & $1.75 E+02$ & $4.6853 E+08$ & $2.1344 E-09$ & & & $1.874 E-03$ \\
\hline Cs 139 & 138.9134 & $9.270 \mathrm{E}+00$ & minute & $5.56 E+02$ & $1.4602 E+08$ & $6.8485 E-09$ & & & $6.282 \mathrm{E}-02$ \\
\hline Cs 140 & 139.9173 & $6.370 \mathrm{E}+01$ & second & $6.37 E+01$ & $1.2658 \mathrm{E}+09$ & $7.9001 E-10$ & & & $5.745 \mathrm{E}-02$ \\
\hline Cs 141 & 140.9200 & $2.494 E+01$ & second & $2.49 E+01$ & $3.2100 \mathrm{E}+09$ & $3.1152 E-10$ & & & $4.424 \mathrm{E}-02$ \\
\hline Cs 142 & 141.9243 & $1.700 \mathrm{E}+00$ & second & $1.70 \mathrm{E}+00$ & $4.6760 \mathrm{E}+10$ & $2.1386 \mathrm{E}-11$ & & & 2.677E-02 \\
\hline Cs 143 & 142.9273 & $1.780 \mathrm{E}+00$ & second & $1.78 \mathrm{E}+00$ & $4.4345 E+10$ & $2.2551 E-11$ & & & $1.481 E-02$ \\
\hline Cs 144 & 143.9320 & $1.010 \mathrm{E}+00$ & second & $1.01 E+00$ & $7.7606 \mathrm{E}+10$ & $1.2886 \mathrm{E}-11$ & & & $3.558 \mathrm{E}-03$ \\
\hline Cs 145 & 144.9354 & $5.940 \mathrm{E}-01$ & second & $5.94 \mathrm{E}-01$ & $1.3104 E+11$ & $7.6311 \mathrm{E}-12$ & & & $5.782 \mathrm{E}-04$ \\
\hline Cs 146 & 145.9402 & $3.210 \mathrm{E}-01$ & second & 3.21E-01 & $2.4082 \mathrm{E}+11$ & $4.1524 \mathrm{E}-12$ & & & $1.684 \mathrm{E}-04$ \\
\hline Cs 147 & 146.9439 & $2.250 \mathrm{E}-01$ & second & $2.25 \mathrm{E}-01$ & $3.4123 E+11$ & $2.9306 E-12$ & & & $5.546 \mathrm{E}-07$ \\
\hline Cs 148 & 147.9489 & $1.580 \mathrm{E}-01$ & second & $1.58 \mathrm{E}-01$ & $4.8262 \mathrm{E}+11$ & $2.0720 E-12$ & & & $7.719 E-08$ \\
\hline
\end{tabular}




\begin{tabular}{|c|c|c|c|c|c|c|c|c|c|}
\hline Isotope & $\begin{array}{l}\text { Atomic } \\
\text { Mass }\end{array}$ & Halflife & $\begin{array}{l}\text { Time } \\
\text { Units }\end{array}$ & $t_{1 / 2}, \sec$ & Curies/ gram & Grams/ Curie & $\begin{array}{c}\text { Watts/Ci } \\
\text { (Ref.3) }\end{array}$ & $\begin{array}{c}\text { Individual }^{1} \\
\text { Fission } \\
\text { Product Yield } \\
\text { (Ref.4) }\end{array}$ & $\begin{array}{c}\text { Cumulative } \\
\text { Fission Product } \\
\text { Yield (Ref.4) }\end{array}$ \\
\hline Ba 117 & 116.9286 & $1.750 E+00$ & second & $1.75 E+00$ & $5.5134 \mathrm{E}+10$ & $1.8138 \mathrm{E}-11$ & & & \\
\hline Ba 119 & 118.9311 & $5.400 E+00$ & second & $5.40 \mathrm{E}+00$ & $1.7567 E+10$ & $5.6926 \mathrm{E}-11$ & & & \\
\hline Ba120 & 119.9260 & $3.200 E+01$ & second & $3.20 E+01$ & $2.9398 E+09$ & $3.4016 \mathrm{E}-10$ & & & \\
\hline $\mathrm{Ba} 121$ & 120.9245 & $2.970 E+01$ & second & $2.97 \mathrm{E}+01$ & $3.1413 \mathrm{E}+09$ & $3.1834 \mathrm{E}-10$ & & & \\
\hline $\mathrm{Ba122}$ & 121.9203 & $1.950 E+00$ & minute & $1.17 \mathrm{E}+02$ & $7.9089 \mathrm{E}+08$ & $1.2644 \mathrm{E}-09$ & & & \\
\hline Ba 123 & 122.9185 & $2.700 E+00$ & minute & $1.62 E+02$ & $5.6656 E+08$ & $1.7650 E-09$ & & & \\
\hline Ba 124 & 123.9151 & $1.190 \mathrm{E}+01$ & minute & $7.14 E+02$ & $1.2751 E+08$ & $7.8423 \mathrm{E}-09$ & & & \\
\hline $\mathrm{Ba} 125$ & 124.9146 & $3.500 E+00$ & minute & $2.10 \mathrm{E}+02$ & $4.3007 E+08$ & $2.3252 \mathrm{E}-09$ & & & \\
\hline $\mathrm{Ba} 125 \mathrm{~m}$ & 124.9146 & $8.000 E+00$ & minute & $4.80 \mathrm{E}+02$ & $1.8816 \mathrm{E}+08$ & $5.3147 \mathrm{E}-09$ & & & \\
\hline Ba 126 & 125.9112 & $1.000 E+02$ & minute & $6.00 \mathrm{E}+03$ & $1.4933 E+07$ & $6.6964 E-08$ & & & \\
\hline Ba 127 & 126.9111 & $1.270 E+01$ & minute & $7.62 E+02$ & $1.1666 \mathrm{E}+08$ & $8.5719 E-09$ & & & \\
\hline Ba $127 \mathrm{~m}$ & 126.9111 & $1.900 E+00$ & second & $1.90 \mathrm{E}+00$ & $4.6787 \mathrm{E}+10$ & $2.1374 \mathrm{E}-11$ & & & \\
\hline Ba 128 & 127.9083 & $2.430 E+00$ & day & $2.10 E+05$ & $4.2010 \mathrm{E}+05$ & $2.3804 \mathrm{E}-06$ & & & \\
\hline Ba 129 & 128.9087 & $2.230 E+00$ & hour & $8.03 E+03$ & $1.0902 E+07$ & $9.1730 \mathrm{E}-08$ & & & \\
\hline $\mathrm{Ba} 129 \mathrm{~m}$ & 128.9087 & $2.170 E+00$ & hour & $7.81 \mathrm{E}+03$ & $1.1203 E+07$ & $8.9262 E-08$ & & & \\
\hline $\mathrm{Ba} 130 \mathrm{~m}$ & 129.9063 & $1.100 \mathrm{E}-02$ & second & $1.10 \mathrm{E}-02$ & $7.8950 E+12$ & $1.2666 \mathrm{E}-13$ & & & \\
\hline $\mathrm{Ba} 131$ & 130.9069 & $1.150 E+01$ & day & $9.94 \mathrm{E}+05$ & $8.6737 E+04$ & $1.1529 \mathrm{E}-05$ & & & \\
\hline Ba $131 \mathrm{~m}$ & 130.9069 & $1.460 E+01$ & minute & $8.76 \mathrm{E}+02$ & $9.8381 E+07$ & $1.0165 \mathrm{E}-08$ & & & \\
\hline Ba 133 & 132.9060 & $1.052 \mathrm{E}+01$ & year & $3.32 E+08$ & $2.5568 \mathrm{E}+02$ & $3.9111 \mathrm{E}-03$ & 0.002722 & & $5.018 \mathrm{E}-13$ \\
\hline $\mathrm{Ba} 133 \mathrm{~m}$ & 132.9060 & $3.890 \mathrm{E}+01$ & hour & $1.40 \mathrm{E}+05$ & $6.0615 E+05$ & $1.6498 \mathrm{E}-06$ & & & $3.844 E-13$ \\
\hline Ba 135m & 134.9057 & $2.870 E+01$ & hour & $1.03 E+05$ & $8.0940 E+05$ & $1.2355 \mathrm{E}-06$ & & & $7.282 \mathrm{E}-09$ \\
\hline $\mathrm{Ba} 136 \mathrm{~m}$ & 135.9046 & $3.084 \mathrm{E}-01$ & second & $3.08 \mathrm{E}-01$ & $2.6917 E+11$ & $3.7151 \mathrm{E}-12$ & & & $8.837 E-06$ \\
\hline $\mathrm{Ba} 137 \mathrm{~m}$ & 136.9058 & $2.552 E+00$ & minute & $1.53 \mathrm{E}+02$ & $5.3817 E+08$ & $1.8581 \mathrm{E}-09$ & 0.003940 & & 5.937E-02 \\
\hline Ba 139 & 138.9088 & $8.306 \mathrm{E}+01$ & minute & $4.98 \mathrm{E}+03$ & $1.6297 E+07$ & $6.1362 E-08$ & & & $6.311 \mathrm{E}-02$ \\
\hline $\mathrm{Ba} 140$ & 139.9106 & $1.275 \mathrm{E}+01$ & day & $1.10 E+06$ & $7.3187 E+04$ & $1.3664 \mathrm{E}-05$ & & & $6.119 \mathrm{E}-02$ \\
\hline $\mathrm{Ba} 141$ & 140.9144 & $1.827 E+01$ & minute & $1.10 E+03$ & $7.3035 E+07$ & $1.3692 \mathrm{E}-08$ & & & $5.938 \mathrm{E}-02$ \\
\hline Ba 142 & 141.9164 & $1.060 E+01$ & minute & $6.36 \mathrm{E}+02$ & $1.2499 \mathrm{E}+08$ & $8.0004 \mathrm{E}-09$ & & & $5.586 \mathrm{E}-02$ \\
\hline Ba 143 & 142.9206 & $1.433 E+01$ & second & $1.43 \mathrm{E}+01$ & $5.5085 \mathrm{E}+09$ & $1.8154 \mathrm{E}-10$ & & & $5.276 \mathrm{E}-02$ \\
\hline 8 a 144 & 143.9229 & $1.150 \mathrm{E}+01$ & second & $1.15 \mathrm{E}+01$ & $6.8163 E+09$ & $1.4671 \mathrm{E}-10$ & & & $4.160 \mathrm{E}-02$ \\
\hline Ba 145 & 144.9269 & $4.310 E+00$ & second & $4.31 \mathrm{E}+00$ & $1.8061 E+10$ & $5.5367 \mathrm{E}-11$ & & & $2.011 \mathrm{E}-02$ \\
\hline $\mathrm{Ba} 146$ & 145.9301 & $2.220 \mathrm{E}+00$ & second & $2.22 \mathrm{E}+00$ & $3.4824 \mathrm{E}+10$ & $2.8716 \mathrm{E}-11$ & & & $7.400 \mathrm{E}-03$ \\
\hline $\mathrm{Ba} 147$ & 146.9340 & $8.930 \mathrm{E}-01$ & second & $8.93 \mathrm{E}-01$ & $8.5981 E+10$ & $1.1630 \mathrm{E}-11$ & & & $1.327 \mathrm{E}-03$ \\
\hline $\mathrm{Ba} 148$ & 147.9377 & $6.070 \mathrm{E}-01$ & second & $6.07 \mathrm{E}-01$ & $1.2563 E+11$ & $7.9596 \mathrm{E}-12$ & & & $1.753 \mathrm{E}-04$ \\
\hline Ba 149 & 148.9425 & $3.440 \mathrm{E}-01$ & second & $3.44 \mathrm{E}-01$ & $2.2019 E+11$ & $4.5415 \mathrm{E}-12$ & & & $1.121 \mathrm{E}-05$ \\
\hline Ba 150 & 149.9456 & 3.000E-01 & second & $3.00 \mathrm{E}-01$ & $2.5080 E+11$ & $3.9873 \mathrm{E}-12$ & & & $5.498 \mathrm{E}-07$ \\
\hline
\end{tabular}




\begin{tabular}{|c|c|c|c|c|c|c|c|c|c|}
\hline isotope & $\begin{array}{l}\text { Atomic } \\
\text { Mass }\end{array}$ & Halflife & $\begin{array}{l}\text { Time } \\
\text { Units }\end{array}$ & $t_{1 / 2}, \sec$ & Curies/ gram & Grams/ Curie & $\begin{array}{c}\text { Watts/Ci } \\
\text { (Ref.3) }\end{array}$ & \begin{tabular}{|c|}
$\begin{array}{c}\text { Individual } \\
\text { Fission }\end{array}$ \\
Product Yield \\
(Ref.4) \\
\end{tabular} & $\begin{array}{l}\text { Cumulative } \\
\text { Fission Product } \\
\text { Yleld (Ref.4) }\end{array}$ \\
\hline La 120 & 119.9381 & $2.800 E+00$ & second & $2.80 E+00$ & $3.3594 \mathrm{E}+10$ & $2.9767 \mathrm{E}-11$ & & & \\
\hline La 121 & 120.9330 & $5.300 \mathrm{E}+00$ & second & $5.30 \mathrm{E}+00$ & $1.7602 \mathrm{E}+10$ & $5.6813 \mathrm{E}-11$ & & & \\
\hline La 122 & 121.9307 & $8.700 E+00$ & second & $8.70 E+00$ & $1.0635 E+10$ & $9.4028 \mathrm{E}-11$ & & & \\
\hline La 123 & 122.9262 & $1.700 \mathrm{E}+01$ & second & $1.70 \mathrm{E}+01$ & $5.3986 \mathrm{E}+09$ & $1.8523 \mathrm{E}-10$ & & & \\
\hline La 124 & 123.9245 & $2.900 \mathrm{E}+01$ & second & $2.90 E+01$ & $3.1392 \mathrm{E}+09$ & $3.1855 \mathrm{E}-10$ & & & \\
\hline La 125 & 124.9207 & $7.600 \mathrm{E}+01$ & second & $7.60 \mathrm{E}+01$ & $1.1883 E+09$ & $8.4153 \mathrm{E}-10$ & & & \\
\hline La 126 & 125.9194 & $5.400 \mathrm{E}+01$ & second & $5.40 E+01$ & $1.6592 E+09$ & $6.0271 \mathrm{E}-10$ & & & \\
\hline La 127 & 126.9162 & $3.800 E+00$ & minute & $2.28 \mathrm{E}+02$ & $3.8987 \mathrm{E}+08$ & $2.5649 \mathrm{E}-09$ & & & \\
\hline La128 & 127.9154 & $5.000 \mathrm{E}+00$ & minute & $3.00 E+02$ & $2.9399 E+08$ & $3.4015 \mathrm{E}-09$ & & & \\
\hline La 129 & 128.9127 & $1.160 \mathrm{E}+01$ & minute & $6.96 \mathrm{E}+02$ & $1.2574 \mathrm{E}+08$ & 7.9530 E-09 & & & \\
\hline La $129 \mathrm{~m}$ & 128.9127 & $5.600 \mathrm{E}-01$ & second & $5.60 \mathrm{E}-01$ & $1.5628 \mathrm{E}+11$ & $6.3989 \mathrm{E}-12$ & & & \\
\hline La 130 & 129.9123 & $8.700 E+00$ & minute & $5.22 E+02$ & $1.6636 E+08$ & $6.0110 \mathrm{E}-09$ & & & \\
\hline La 131 & 130.9101 & $5.900 \mathrm{E}+01$ & minute & $3.54 E+03$ & $2.4344 E+07$ & $4.1077 \mathrm{E}-08$ & & & \\
\hline La 132 & 131.9101 & $4.800 E+00$ & hour & $1.73 E+04$ & $4.9494 E+06$ & $2.0204 \mathrm{E}-07$ & & & \\
\hline La $132 \mathrm{~m}$ & 131.9101 & $2.430 E+01$ & minute & $1.46 E+03$ & $5.8660 \mathrm{E}+07$ & $1.7047 \mathrm{E}-08$ & & & \\
\hline La 133 & 132.9084 & $3.912 E+00$ & hour & $1.41 E+04$ & $6.0273 E+06$ & $1.6591 \mathrm{E}-07$ & & & \\
\hline La 134 & 133.9085 & $6.450 \mathrm{E}+00$ & minute & $3.87 E+02$ & $2.1770 E+08$ & $4.5935 \mathrm{E}-09$ & & & \\
\hline La 135 & 134.9070 & $1.950 \mathrm{E}+01$ & hour & $7.02 E+04$ & $1.1913 E+06$ & $8.3945 E-07$ & & & \\
\hline La 136 & 135.9077 & $9.870 E+00$ & minute & $5.92 E+02$ & $1.4017 \mathrm{E}+08$ & 7.1340 E-09 & & & \\
\hline La $136 \mathrm{~m}$ & 135.9077 & $1.140 \mathrm{E}-01$ & second & $1.14 \mathrm{E}-01$ & $7.2816 \mathrm{E}+11$ & $1.3733 \mathrm{E}-12$ & & & \\
\hline La 137 & 136.9065 & $6.000 \mathrm{E}+04$ & year & $1.89 E+12$ & $4.3520 \mathrm{E}-02$ & $2.2978 \mathrm{E}+01$ & & & $1.080 \mathrm{E}-07$ \\
\hline La 138 & 137.9071 & $1.050 E+11$ & year & $3.31 E+18$ & $2.4688 \mathrm{E}-08$ & $4.0506 \mathrm{E}+07$ & & & $6.312 \mathrm{E}-02$ \\
\hline La 140 & 139.9095 & $1.678 E+00$ & day & $1.45 E+05$ & $5.5616 \mathrm{E}+05$ & $1.7981 \mathrm{E}-06$ & & & $6.120 E-02$ \\
\hline La 141 & 140.9110 & $3.920 \mathrm{E}+00$ & hour & $1.41 E+04$ & $5.6734 E+06$ & $1.7626 \mathrm{E}-07$ & & & $5.954 \mathrm{E}-02$ \\
\hline La 142 & 141.9141 & $9.110 E+01$ & minute & $5.47 E+03$ & $1.4544 E+07$ & $6.8757 \mathrm{E}-08$ & & & $5.670 \mathrm{E}-02$ \\
\hline La 143 & 142.9161 & $1.420 \mathrm{E}+01$ & minute & $8.52 E+02$ & $9.2652 E+07$ & $1.0793 \mathrm{E}-08$ & & & $5.684 \mathrm{E}-02$ \\
\hline La 144 & 143.9196 & $4.080 \mathrm{E}+01$ & second & $4.08 E+01$ & $1.9213 E+09$ & $5.2048 \mathrm{E}-10$ & & & $5.218 \mathrm{E}-02$ \\
\hline La 145 & 144.9216 & $2.480 E+01$ & second & $2.48 E+01$ & $3.1390 \mathrm{E}+09$ & $3.1857 \mathrm{E}-10$ & & & $3.575 \mathrm{E}-02$ \\
\hline La 146 & 145.9257 & $6.270 E+00$ & second & $6.27 E+00$ & $1.2330 E+10$ & $8.1100 \mathrm{E}-11$ & & & $1.074 \mathrm{E}-02$ \\
\hline La $146 \mathrm{~m}$ & 145.9257 & $1.000 \mathrm{E}+01$ & second & $1.00 E+01$ & $7.7312 \mathrm{E}+09$ & $1.2935 \mathrm{E}-10$ & & & $1.165 \mathrm{E}-02$ \\
\hline La 147 & 146.9278 & $4.015 \mathrm{E}+00$ & second & $4.02 E+00$ & $1.9124 \mathrm{E}+10$ & $5.2289 \mathrm{E}-11$ & & & $1.130 \mathrm{E}-02$ \\
\hline La 148 & 147.9322 & $1.428 \mathrm{E}+00$ & second & $1.43 E+00$ & $5.3405 E+10$ & $1.8725 E-11$ & & & $3.803 E-03$ \\
\hline La 149 & 148.9344 & $1.050 \mathrm{E}+00$ & second & $1.05 E+00$ & $7.2143 E+10$ & $1.3861 \mathrm{E}-11$ & & & $8.424 E-04$ \\
\hline La 150 & 149.9386 & $8.600 \mathrm{E}-01$ & second & $8.60 \mathrm{E}-01$ & $8.7491 \mathrm{E}+10$ & $1.1430 \mathrm{E}-11$ & & & $1.205 \mathrm{E}-04$ \\
\hline Ce 123 & 122.9355 & $3.200 \mathrm{E}+00$ & second & $3.20 E+00$ & $2.8678 E+10$ & $3.4870 E-11$ & & & \\
\hline
\end{tabular}

WSRC-TR-99-00344

October, 1999

page 36 
Radionuclide Specific Data

\begin{tabular}{|c|c|c|c|c|c|c|c|c|c|}
\hline Isotope & $\begin{array}{l}\text { Atomic } \\
\text { Mass }\end{array}$ & Halflife & $\begin{array}{l}\text { Time } \\
\text { Units }\end{array}$ & $t_{1 / 2}, \sec$ & Curies/gram & Grams/ Curie & $\begin{array}{c}\text { Watts/Ci } \\
\text { (Ref.3) }\end{array}$ & $\begin{array}{c}\text { Individual }^{4} \\
\text { Fission } \\
\text { Product Yield } \\
\text { (Ref.4) } \\
\end{array}$ & $\begin{array}{l}\text { Cumulative } \\
\text { Fission Product } \\
\text { Yield (Ref.4) }\end{array}$ \\
\hline Ce 124 & 123.9305 & $6.000 E+00$ & second & $6.00 \mathrm{E}+00$ & $1.5172 \mathrm{E}+10$ & $6.5910 \mathrm{E}-11$ & & & \\
\hline Ce 125 & 124.9285 & $9.000 E+00$ & second & $9.00 \mathrm{E}+00$ & $1.0034 \mathrm{E}+10$ & $9.9662 \mathrm{E}-11$ & & & \\
\hline $\mathrm{Ce} 126$ & 125.9241 & $5.000 E+01$ & second & $5.00 E+01$ & $1.7918 \mathrm{E}+09$ & $5.5809 \mathrm{E}-10$ & & & \\
\hline Ce 127 & 126.9228 & $3.200 \mathrm{E}+01$ & second & $3.20 \mathrm{E}+01$ & $2.7777 \mathrm{E}+09$ & $3.6001 \mathrm{E}-10$ & & & \\
\hline Ce 128 & 127.9189 & $3.000 E+00$ & minutes & $1.80 E+02$ & $4.8997 \mathrm{E}+08$ & $2.0409 \mathrm{E}-09$ & & & \\
\hline $\mathrm{Ce} 129$ & 128.9181 & $3.500 E+00$ & minute & $2.10 E+02$ & $4.1672 E+08$ & $2.3997 E-09$ & & & \\
\hline $\mathrm{Ce} 130$ & 129.9147 & $2.500 \mathrm{E}+01$ & minute & $1.50 \mathrm{E}+03$ & $5.7893 \mathrm{E}+07$ & $1.7273 E-08$ & & & \\
\hline $\mathrm{Ce} 131$ & 130.9144 & $1.020 \mathrm{E}+01$ & minute & $6.12 E+02$ & $1.4081 \mathrm{E}+08$ & $7.1017 \mathrm{E}-09$ & & & \\
\hline $\mathrm{Ce} 131 \mathrm{~m}$ & 130.9144 & $5.000 E+00$ & minute & $3.00 \mathrm{E}+02$ & $2.8726 \mathrm{E}+08$ & $3.4812 E-09$ & & & \\
\hline Ce 132 & 131.9115 & $3.510 E+00$ & hour & $1.26 E+04$ & $6.7684 \mathrm{E}+06$ & $1.4775 E-07$ & & & \\
\hline Ce $132 m$ & 131.9115 & $1.300 \mathrm{E}-02$ & second & $1.30 \mathrm{E}-02$ & $6.5789 \mathrm{E}+12$ & $1.5200 E-13$ & & & \\
\hline Ce 133 & 132.9116 & $4.900 \mathrm{E}+00$ & hour & $1.76 \mathrm{E}+04$ & $4.8119 \mathrm{E}+06$ & 2.0782E-07 & & & \\
\hline $\mathrm{Ce} 133 \mathrm{~m}$ & 132.9116 & $9.700 \mathrm{E}+01$ & minute & $5.82 E+03$ & $1.4584 \mathrm{E}+07$ & $6.8566 \mathrm{E}-08$ & & & \\
\hline Ce 134 & 133.9090 & $7.590 E+01$ & hour & $2.73 E+05$ & $3.0833 E+05$ & $3.2432 E-06$ & & & \\
\hline Ce 135 & 134.9091 & $1.770 E+01$ & hour & $6.37 E+04$ & $1.3124 E+06$ & $7.6197 \mathrm{E}-07$ & & & \\
\hline Ce $135 \mathrm{~m}$ & 134.9091 & $2.000 \mathrm{E}+01$ & second & $2.00 \mathrm{E}+01$ & $4.1812 E+09$ & $2.3916 \mathrm{E}-10$ & & & \\
\hline Ce 137 & 136.9078 & $9.000 \mathrm{E}+00$ & hour & $3.24 E+04$ & $2.5433 E+06$ & $3.9318 \mathrm{E}-07$ & & & \\
\hline $\mathrm{Ce} 137 \mathrm{~m}$ & 136.9078 & $3.440 \mathrm{E}+01$ & hour & $1.24 E+05$ & $6.6541 \mathrm{E}+05$ & $1.5028 \mathrm{E}-06$ & & & \\
\hline Ce $138 \mathrm{~m}$ & 137.9060 & $8.650 E-03$ & second & $8.65 \mathrm{E}-03$ & $9.4575 E+12$ & $1.0574 \mathrm{E}-13$ & & & \\
\hline Ce 139 & 138.9066 & $1.376 E+02$ & day & $1.19 E+07$ & $6.8296 \mathrm{E}+03$ & $1.4642 \mathrm{E}-04$ & & & $6.727 \mathrm{E}-11$ \\
\hline Ce $139 m$ & 138.9066 & $5.480 E+01$ & second & $5.48 \mathrm{E}+01$ & $1.4821 \mathrm{E}+09$ & $6.7473 \mathrm{E}-10$ & & & $4.956 E-11$ \\
\hline Ce 141 & 140.9083 & $3.250 E+01$ & day & $2.81 \mathrm{E}+06$ & $2.8512 E+04$ & 3.5073E-05 & 0.001470 & & $5.954 \mathrm{E}-02$ \\
\hline $\mathrm{Ce} 142$ & 141.9092 & $5.000 E+16$ & year & $1.58 E+24$ & $5.0383 \mathrm{E}-14$ & $1.9848 \mathrm{E}+13$ & & & $5.670 E-02$ \\
\hline Ce 143 & 142.9124 & $3.304 E+01$ & hour & $1.19 \mathrm{E}+05$ & $6.6371 \mathrm{E}+05$ & 1.5067E-06 & & & $5.689 \mathrm{E}-02$ \\
\hline Ce 144 & 143.9136 & $2.849 E+02$ & day & $2.46 E+07$ & $3.1848 \mathrm{E}+03$ & $3.1399 \mathrm{E}-04$ & 0.000658 & & $5.258 \mathrm{E}-02$ \\
\hline Ce 145 & 144.9172 & $3.010 E+00$ & minute & $1.81 \mathrm{E}+02$ & $4.3106 \mathrm{E}+08$ & $2.3199 \mathrm{E}-09$ & & & $3.742 E-02$ \\
\hline $\mathrm{Ce} 146$ & 145.9187 & $1.352 E+01$ & minute & $8.11 \mathrm{E}+02$ & $9.5310 \mathrm{E}+07$ & $1.0492 \mathrm{E}-08$ & & & $2.896 \mathrm{E}-02$ \\
\hline $\mathrm{Ce} 147$ & 146.9225 & $5.640 E+01$ & second & $5.64 E+01$ & $1.3615 \mathrm{E}+09$ & $7.3450 \mathrm{E}-10$ & & & $2.049 \mathrm{E}-02$ \\
\hline $\begin{array}{ll}\mathrm{Ce} 148 \\
\end{array}$ & 147.9244 & $5.600 \mathrm{E}+01$ & second & $5.60 E+01$ & $1.3619 \mathrm{E}+09$ & $7.3426 \mathrm{E}-10$ & & & $1.524 \mathrm{E}-02$ \\
\hline Ce 149 & 148.9283 & $5.300 \mathrm{E}+00$ & second & $5.30 \mathrm{E}+00$ & $1.4293 \mathrm{E}+10$ & $6.9964 \mathrm{E}-11$ & & & $7.434 E-03$ \\
\hline $\mathrm{Ce} 150$ & 149.9302 & $4.000 \mathrm{E}+00$ & second & $4.00 \mathrm{E}+00$ & $1.8812 E+10$ & $5.3158 \mathrm{E}-11$ & & & $3.265 \mathrm{E}-03$ \\
\hline Ce 151 & 150.9340 & $1.020 E+00$ & second & $1.02 E+00$ & $7.3281 E+10$ & $1.3646 \mathrm{E}-11$ & & & $8.218 E-04$ \\
\hline $\operatorname{Pr} 121$ & 120.9554 & $1.400 \mathrm{E}+00$ & second & $1.40 \mathrm{E}+00$ & $6.6623 \mathrm{E}+10$ & $1.5010 \mathrm{E}-11$ & & & \\
\hline $\operatorname{Pr} 126$ & 125.9353 & $3.100 E+00$ & second & $3.10 \mathrm{E}+00$ & $2.8898 \mathrm{E}+10$ & $3.4605 \mathrm{E}-11$ & & & \\
\hline Pr 127 & 126.9308 & $4.900 \mathrm{E}+00$ & second & $4.90 E+00$ & $1.8139 \mathrm{E}+10$ & $5.5130 \mathrm{E}-11$ & & & \\
\hline
\end{tabular}

WSRC-TR-99-00344

October, 1999

page 37 


\begin{tabular}{|c|c|c|c|c|c|c|c|c|c|}
\hline Isotope & $\begin{array}{l}\text { Atomic } \\
\text { Mass }\end{array}$ & Halflife & $\begin{array}{l}\text { Time } \\
\text { Units }\end{array}$ & $t_{1 / 2}, \sec$ & Curies/gram & Grams/ Curie & $\begin{array}{c}\text { Watts/Ci } \\
\text { (Ref.3) }\end{array}$ & $\begin{array}{c}\text { Individual }^{1} \\
\text { Fission } \\
\text { Product Yield } \\
\text { (Ref.4) }\end{array}$ & $\begin{array}{c}\text { Cumulative } \\
\text { Fission Product } \\
\text { Yield (Ref.4) }\end{array}$ \\
\hline $\operatorname{Pr} 128$ & 127.9288 & $3.100 \mathrm{E}+00$ & second & $3.10 E+00$ & $2.8448 \mathrm{E}+10$ & $3.5152 \mathrm{E}-11$ & & & \\
\hline $\operatorname{Pr} 129$ & 128.9249 & $2.400 E+01$ & second & $2.40 \mathrm{E}+01$ & $3.6461 \mathrm{E}+09$ & $2.7427 \mathrm{E}-10$ & & & \\
\hline $\operatorname{Pr} 130$ & 129.9234 & $4.000 \mathrm{E}+01$ & second & $4.00 E+01$ & $2.1708 E+09$ & $4.6065 \mathrm{E}-10$ & & & \\
\hline $\operatorname{Pr} 131$ & 130.9201 & $1.530 \mathrm{E}+00$ & minute & $9.18 E+01$ & $9.3870 \mathrm{E}+08$ & $1.0653 \mathrm{E}-09$ & & & \\
\hline Pr $131 \mathrm{~m}$ & 130.9201 & $5.700 E+00$ & second & $5.70 \mathrm{E}+00$ & $1.5118 \mathrm{E}+10$ & $6.6146 \mathrm{E}-11$ & & & \\
\hline Pr 132 & 131.9191 & $1.600 \mathrm{E}+00$ & minute & $9.60 \mathrm{E}+01$ & $8.9084 E+08$ & $1.1225 \mathrm{E}-09$ & & & \\
\hline Pr 133 & 132.9162 & $6.500 E+00$ & minute & $3.90 \mathrm{E}+02$ & $2.1764 E+08$ & $4.5948 \mathrm{E}-09$ & & & \\
\hline Pr 134 & 133.9157 & $1.700 \mathrm{E}+01$ & minute & $1.02 E+03$ & $8.2593 E+07$ & $1.2108 \mathrm{E}-08$ & & & \\
\hline Pr $134 m$ & 133.9157 & $1.100 \mathrm{E}+01$ & minute & $6.60 \mathrm{E}+02$ & $1.2764 E+08$ & $7.8343 \mathrm{E}-09$ & & & \\
\hline Pr 135 & 134.9131 & $2.400 \mathrm{E}+01$ & minute & $1.44 E+03$ & $5.8071 E+07$ & $1.7220 \mathrm{E}-08$ & & & \\
\hline $\operatorname{Pr} 136$ & 135.9126 & $1.310 \mathrm{E}+01$ & minute & $7.86 E+02$ & $1.0561 E+08$ & $9.4690 \mathrm{E}-09$ & & & \\
\hline Pr 137 & 136.9107 & $1.280 E+00$ & hour & $4.61 \mathrm{E}+03$ & $1.7882 E+07$ & $5.5921 E-08$ & & & \\
\hline Pr 138 & 137.9107 & $1.450 \mathrm{E}+00$ & minute & $8.70 E+01$ & $9.4028 \mathrm{E}+08$ & $1.0635 \mathrm{E}-09$ & & & \\
\hline $\operatorname{Pr} 138 \mathrm{~m}$ & 137.9107 & $2.120 \mathrm{E}+00$ & hour & $7.63 \mathrm{E}+03$ & $1.0719 \mathrm{E}+07$ & $9.3295 \mathrm{E}-08$ & & & \\
\hline Pr 139 & 138.9089 & $4.410 \mathrm{E}+00$ & hour & $1.59 E+04$ & $5.1157 E+06$ & $1.9548 E-07$ & & & \\
\hline $\operatorname{Pr} 140$ & 139.9091 & $3.390 \mathrm{E}+00$ & minute & $2.03 E+02$ & $3.9644 E+08$ & $2.5224 \mathrm{E}-09$ & & & \\
\hline $\operatorname{Pr} 142$ & 141.9100 & $1.912 E+01$ & hour & $6.88 E+04$ & $1.1550 \mathrm{E}+06$ & $8.6582 \mathrm{E}-07$ & & & $3.538 \mathrm{E}-10$ \\
\hline $\operatorname{Pr} 142 m$ & 141.9100 & $1.460 \mathrm{E}+01$ & minute & $8.76 \mathrm{E}+02$ & $9.0753 E+07$ & $1.1019 \mathrm{E}-08$ & & & $2.589 \mathrm{E}-10$ \\
\hline $\operatorname{Pr} 143$ & 142.9108 & $1.357 E+01$ & day & $1.17 \mathrm{E}+06$ & $6.7331 \mathrm{E}+04$ & $1.4852 \mathrm{E}-05$ & 0.001870 & & $5.689 \mathrm{E}-02$ \\
\hline $\operatorname{Pr} 144$ & 143.9133 & $1.728 \mathrm{E}+01$ & minute & $1.04 E+03$ & $7.5610 E+07$ & $1.3226 \mathrm{E}-08$ & 0.007338 & & $5.258 \mathrm{E}-02$ \\
\hline $\operatorname{Pr} 144 m$ & 143.9133 & $7.200 \mathrm{E}+00$ & minute & $4.32 E+02$ & $1.8146 E+08$ & $5.5107 \mathrm{E}-09$ & 0.000340 & & $7.944 \mathrm{E}-04$ \\
\hline Pr 145 & 144.9145 & $5.984 \mathrm{E}+00$ & hour & $2.15 E+04$ & $3.6139 \mathrm{E}+06$ & $2.7671 \mathrm{E}-07$ & & & $3.743 E-02$ \\
\hline $\operatorname{Pr} 146$ & 145.9176 & $2.415 \mathrm{E}+01$ & minute & $1.45 \mathrm{E}+03$ & $5.3358 \mathrm{E}+07$ & $1.8741 \mathrm{E}-08$ & & & $2.903 E-02$ \\
\hline Pr 147 & 146.9190 & $1.340 \mathrm{E}+01$ & minute & $8.04 E+02$ & $9.5509 \mathrm{E}+07$ & $1.0470 \mathrm{E}-08$ & & & $2.096 \mathrm{E}-02$ \\
\hline $\operatorname{Pr} 148$ & 147.9222 & $2.270 \mathrm{E}+00$ & minute & $1.36 \mathrm{E}+02$ & $5.5997 \mathrm{E}+08$ & 1.7858E-09 & & & $1.578 \mathrm{E}-02$ \\
\hline $\operatorname{Pr} 148 \mathrm{~m}$ & 147.9222 & $2.000 \mathrm{E}+00$ & minute & $1.20 E+02$ & $6.3557 E+08$ & $1.5734 \mathrm{E}-09$ & & & $9.093 \mathrm{E}-04$ \\
\hline Pr 149 & 148.9238 & $2.260 \mathrm{E}+00$ & minute & $1.36 E+02$ & $5.5867 E+08$ & 1.7900E-09 & & & $1.013 \mathrm{E}-02$ \\
\hline $\operatorname{Pr} 150$ & 149.9270 & $6.190 \mathrm{E}+00$ & second & $6.19 \mathrm{E}+00$ & $1.2156 \mathrm{E}+10$ & $8.2261 \mathrm{E}-11$ & & & $6.334 \mathrm{E}-03$ \\
\hline Pr 151 & 150.9282 & $1.890 \mathrm{E}+01$ & second & $1.89 \mathrm{E}+01$ & $3.9550 \mathrm{E}+09$ & $2.5285 E-10$ & & & $3.125 \mathrm{E}-03$ \\
\hline $\operatorname{Pr} 152$ & 151.9316 & $3.240 \mathrm{E}+00$ & second & $3.24 \mathrm{E}+00$ & $2.2918 E+10$ & $4.3633 \mathrm{E}-11$ & & & $1.326 \mathrm{E}-03$ \\
\hline Pr 153 & 152.9337 & $4.300 E+00$ & second & $4.30 \mathrm{E}+00$ & $1.7156 \mathrm{E}+10$ & $5.8290 \mathrm{E}-11$ & & & $4.431 \mathrm{E}-04$ \\
\hline Pr 154 & 153.9374 & $2.300 E+00$ & second & $2.30 E+00$ & $3.1864 E+10$ & $3.1383 \mathrm{E}-11$ & & & $6.061 \mathrm{E}-05$ \\
\hline Nd 127 & 126.9405 & $1.800 \mathrm{E}+00$ & second & $1.80 E+00$ & $4.9375 E+10$ & $2.0253 \mathrm{E}-11$ & & & \\
\hline Nd 128 & 127.9354 & $4.000 E+00$ & second & $4.00 \mathrm{E}+00$ & $2.2046 E+10$ & $4.5360 \mathrm{E}-11$ & & & \\
\hline $\begin{array}{ll}\text { Nd } 129 \\
\end{array}$ & 128.9249 & $4.900 \mathrm{E}+00$ & second & $4.90 E+00$ & $1.7858 \mathrm{E}+10$ & $5.5996 \mathrm{E}-11$ & & & \\
\hline
\end{tabular}

WSRC-TR-99-00344

October, 1999

page 38 
Radionuclide Specific Data

\begin{tabular}{|c|c|c|c|c|c|c|c|c|c|}
\hline Isotope & $\begin{array}{l}\text { Atomic } \\
\text { Mass }\end{array}$ & Halflife & $\begin{array}{l}\text { Time } \\
\text { Units }\end{array}$ & $\mathrm{t}_{1 / 2}, \mathrm{sec}$ & Curies/ gram & Grams/ Curie & $\begin{array}{c}\text { Watts/Ci } \\
\text { (Ref.3) }\end{array}$ & \begin{tabular}{|c|} 
Individual $^{1}$ \\
Fission \\
Product Yield \\
(Ref.4) \\
\end{tabular} & $\begin{array}{c}\text { Cumulative } \\
\text { Fission Product } \\
\text { Yield (Ref.4) }\end{array}$ \\
\hline Nd 130 & 129.9288 & $2.800 \mathrm{E}+01$ & second & $2.80 E+01$ & $3.1011 \mathrm{E}+09$ & $3.2247 \mathrm{E}-10$ & & & \\
\hline Nd 131 & 130.9201 & $2.700 \mathrm{E}+01$ & second & $2.70 E+01$ & $3.1916 \mathrm{E}+09$ & $3.1332 \mathrm{E}-10$ & & & \\
\hline Nd 132 & 131.9231 & $1.750 \mathrm{E}+00$ & minute & $1.05 E+02$ & $8.1445 E+08$ & $1.2278 \mathrm{E}-09$ & & & \\
\hline Nd 133 & 132.9222 & $7.000 \mathrm{E}+01$ & second & $7.00 \mathrm{E}+01$ & $1.2125 \mathrm{E}+09$ & $8.2474 \mathrm{E}-10$ & & & \\
\hline Nd $133 \mathrm{~m}$ & 132.9222 & $7.000 \mathrm{E}+01$ & second & $7.00 E+01$ & $1.2125 \mathrm{E}+09$ & $8.2474 E-10$ & & & \\
\hline Nd 134 & 133.9186 & $8.500 \mathrm{E}+00$ & minute & $5.10 E+02$ & $1.6518 E+08$ & $6.0539 \mathrm{E}-09$ & & & \\
\hline Nd 135 & 134.9182 & $1.240 \mathrm{E}+01$ & minute & $7.44 E+02$ & $1.1239 E+08$ & $8.8975 \mathrm{E}-09$ & & & \\
\hline Nd $135 \mathrm{~m}$ & 134.9182 & $5.500 \mathrm{E}+00$ & minute & $3.30 E+02$ & $2.5339 E+08$ & $3.9465 \mathrm{E}-09$ & & & \\
\hline Nd 136 & 135.9150 & $5.065 \mathrm{E}+01$ & minute & $3.04 \mathrm{E}+03$ & $2.7314 \mathrm{E}+07$ & $3.6612 \mathrm{E}-08$ & & & \\
\hline Nd 137 & 136.9146 & $3.850 \mathrm{E}+01$ & minute & $2.31 \mathrm{E}+03$ & $3.5671 E+07$ & $2.8034 E-08$ & & & \\
\hline $\mathrm{Nd} 137 \mathrm{~m}$ & 136.9146 & $1.600 \mathrm{E}+00$ & second & $1.60 \mathrm{E}+00$ & $5.1500 \mathrm{E}+10$ & $1.9418 \mathrm{E}-11$ & & & \\
\hline Nd 138 & 137.9119 & $5.040 \mathrm{E}+00$ & hour. & $1.81 E+04$ & $4.5086 \mathrm{E}+06$ & $2.2180 E-07$ & & & \\
\hline Nd 139 & 138.9119 & $2.970 E+01$ & minute & $1.78 E+03$ & $4.5575 \mathrm{E}+07$ & $2.1942 \mathrm{E}-08$ & & & \\
\hline $\mathrm{Nd} 139 \mathrm{~m}$ & 138.9119 & $5.500 \mathrm{E}+00$ & hour & $1.98 \mathrm{E}+04$ & $4.1018 E+06$ & $2.4380 \mathrm{E}-07$ & & & \\
\hline Nd 140 & 139.9093 & $3.370 \mathrm{E}+00$ & day & $2.91 \mathrm{E}+05$ & $2.7694 \mathrm{E}+05$ & $3.6109 \mathrm{E}-06$ & & & \\
\hline Nd 141 & 140.9096 & $2.490 E+00$ & hour & $8.96 \mathrm{E}+03$ & $8.9317 E+06$ & $1.1196 \mathrm{E}-07$ & & & $1.480 \mathrm{E}-17$ \\
\hline Nd 141m & 140.9096 & $6.200 \mathrm{E}+01$ & second & $6.20 E+01$ & $1.2914 \mathrm{E}+09$ & $7.7438 E-10$ & & & \\
\hline Nd 144 & 143.9101 & $2.290 \mathrm{E}+15$ & year & $7.23 \mathrm{E}+22$ & $1.0848 \mathrm{E}-12$ & $9.2186 E+11$ & & & $5.258 \mathrm{E}-02$ \\
\hline Nd 147 & 146.9161 & $1.098 \mathrm{E}+01$ & day & $9.49 E+05$ & $8.0945 E+04$ & $1.2354 \mathrm{E}-05$ & & & $2.096 \mathrm{E}-02$ \\
\hline Nd 149 & 148.9201 & $1.728 E+00$ & hour & $6.22 E+03$ & $1.2178 E+07$ & $8.2115 \mathrm{E}-0.8$ & & & $1.026 \mathrm{E}-02$ \\
\hline Nd 150 & 149.9209 & $1.100 \mathrm{E}+19$ & year & $3.47 \mathrm{E}+26$ & $2.1677 \mathrm{E}-16$ & $4.6131 \mathrm{E}+15$ & & & $6.841 \mathrm{E}-03$ \\
\hline Nd 151 & 150.9238 & $1.244 \mathrm{E}+01$ & minute & $7.46 \mathrm{E}+02$ & $1.0015 \mathrm{E}+08$ & $9.9851 \mathrm{E}-09$ & & & $4.060 \mathrm{E}-03$ \\
\hline Nd 152 & 151.9247 & $1.140 \mathrm{E}+01$ & minute & $6.84 E+02$ & $1.0857 \mathrm{E}+08$ & $9.2110 \mathrm{E}-09$ & & & $2.733 \mathrm{E}-03$ \\
\hline Nd 153 & 152.9277 & $2.890 \mathrm{E}+01$ & second & $2.89 \mathrm{E}+01$ & $2.5527 \mathrm{E}+09$ & $3.9175 \mathrm{E}-10$ & & & $1.579 \mathrm{E}-03$ \\
\hline Nd 154 & 153.9295 & $2.590 \mathrm{E}+01$ & second & $2.59 \mathrm{E}+01$ & $2.8298 \mathrm{E}+09$ & $3.5338 \mathrm{E}-10$ & & & $5.637 \mathrm{E}-04$ \\
\hline Nd 155 & 154.9327 & $8.900 E+00$ & second & $8.90 E+00$ & $8.1817 \mathrm{E}+09$ & $1.2222 \mathrm{E}-10$ & & & $2.824 \mathrm{E}-04$ \\
\hline Nd 156 & 155.9441 & $5.470 E+00$ & second & $5.47 \mathrm{E}+00$ & $1.3226 \mathrm{E}+10$ & $7.5610 \mathrm{E}-11$ & & & $5.770 \mathrm{E}-05$ \\
\hline Pm 130 & 129.9405 & $2.200 \mathrm{E}+00$ & second & $2.20 \mathrm{E}+00$ & $3.9465 \mathrm{E}+10$ & $2.5339 \mathrm{E}-11$ & & & 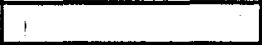 \\
\hline Pm 132 & 131.9338 & $6.300 \mathrm{E}+00$ & second & $6.30 \mathrm{E}+00$ & $1.3573 E+10$ & $7.3675 E-11$ & & & \\
\hline Pm 133 & 132.9297 & $1.500 \mathrm{E}+01$ & second & $1.50 \mathrm{E}+01$ & $5.6580 \mathrm{E}+09$ & $1.7674 \mathrm{E}-10$ & & & \\
\hline $\mathrm{Pm} 134$ & 133.9285 & $2.400 \mathrm{E}+01$ & second & $2.40 \mathrm{E}+01$ & $3.5099 \mathrm{E}+09$ & $2.8491 \mathrm{E}-10$ & & & \\
\hline Pm 135 & 134.9246 & $4.900 E+01$ & second & $4.90 E+01$ & $1.7064 \mathrm{E}+09$ & $5.8602 \mathrm{E}-10$ & & & \\
\hline Pm 136 & 135.9234 & $1.070 E+02$ & second & $1.07 \mathrm{E}+02$ & $7.7571 \mathrm{E}+08$ & $1.2891 \mathrm{E}-09$ & & & \\
\hline $\mathrm{Pm} \mathrm{136m}$ & 135.9234 & $4.700 \mathrm{E}+01$ & second & $4.70 \mathrm{E}+01$ & $1.7660 \mathrm{E}+09$ & $5.6626 \mathrm{E}-10$ & & & \\
\hline $\mathrm{Pm} 137$ & 136.9207 & $2.400 \mathrm{E}+00$ & minute & $1.44 E+02$ & $5.7220 E+08$ & $1.7477 \mathrm{E}-09$ & & & \\
\hline
\end{tabular}

WSRC-TR-99-00344

October, 1999

page 39 


\begin{tabular}{|c|c|c|c|c|c|c|c|c|c|}
\hline Isotope & $\begin{array}{c}\text { Atomic } \\
\text { Mass }\end{array}$ & Halflife & $\begin{array}{l}\text { Time } \\
\text { Units }\end{array}$ & $t_{1 / R}, \sec$ & Curies/gram & Grams/ Curie & $\begin{array}{c}\text { Watts/Ci } \\
\text { (Ref.3) }\end{array}$ & $\begin{array}{c}\text { Individual }^{1} \\
\text { Fission } \\
\text { Product Yield } \\
\text { (Ref.4) }\end{array}$ & $\begin{array}{c}\text { Cumulative }^{1} \\
\text { Fission Product } \\
\text { Yleld (Ref.4) }\end{array}$ \\
\hline Pm 138 & 137.9194 & $1.000 \mathrm{E}+01$ & second & $1.00 \mathrm{E}+01$ & $8.1800 E+09$ & $1.2225 \mathrm{E}-10$ & & & \\
\hline$P m 138 m$ & 137.9194 & $3.240 E+00$ & minute & $1.94 \mathrm{E}+02$ & $4.2078 E+08$ & $2.3765 \mathrm{E}-09$ & & & \\
\hline $\mathrm{Pm} 138 \mathrm{~m} 2$ & 137.9194 & $3.240 \mathrm{E}+00$ & minute & $1.94 E+02$ & $4.2078 E+08$ & $2.3765 \mathrm{E}-09$ & & & \\
\hline Pm 139 & 138.9168 & $4.150 \mathrm{E}+00$ & minute & $2.49 E+02$ & $3.2615 E+08$ & 3.0660 E-09 & & & \\
\hline Pm 139m & 138.9168 & $1.800 \mathrm{E}-01$ & second & $1.80 E-01$ & $4.5118 \mathrm{E}+11$ & $2.2164 E-12$ & & & \\
\hline Pm 140 & 139.9158 & $9.200 E+00$ & second & $9.20 \mathrm{E}+00$ & $8.7644 \mathrm{E}+09$ & $1.1410 \mathrm{E}-10$ & & & \\
\hline $\mathrm{Pm} \mathrm{140m}$ & 139.9158 & $5.950 E+00$ & minute & $3.57 E+02$ & $2.2586 E+08$ & $4.4275 \mathrm{E}-09$ & & & \\
\hline Pm 141 & 140.9136 & $2.090 E+01$ & minute & $1.25 E+03$ & $6.3845 \mathrm{E}+07$ & $1.5663 E-08$ & & & \\
\hline Pm 142 & 141.9130 & $4.050 \mathrm{E}+01$ & second & $4.05 E+01$ & $1.9629 E+09$ & $5.0945 E-10$ & & & \\
\hline $\mathrm{Pm} 143$ & 142.9109 & $2.650 E+02$ & day & $2.29 E+07$ & $3.4479 E+03$ & $2.9003 E-04$ & & & \\
\hline $\mathrm{Pm} 144$ & 143.9126 & $3.630 \mathrm{E}+02$ & day & $3.14 \mathrm{E}+07$ & $2.4995 E+03$ & $4.0008 E-04$ & & & $1.236 \mathrm{E}-16$ \\
\hline $\mathrm{Pm} 145$ & 144.9127 & $1.770 E+01$ & year & $5.59 E+08$ & $1.3937 E+02$ & 7.1750 E-03 & & & $1.889 \mathrm{E}-14$ \\
\hline $\mathrm{Pm} 146$ & 145.9147 & $5.530 E+00$ & year & $1.75 E+08$ & $4.4303 \mathrm{E}+02$ & $2.2572 E-03$ & 0.005020 & & $2.579 E-12$ \\
\hline $\mathrm{Pm} 147$ & 146.9151 & $2.623 E+00$ & year & $8.28 E+07$ & $9.2753 E+02$ & $1.0781 \mathrm{E}-03$ & 0.000370 & & $2.096 \mathrm{E}-02$ \\
\hline$P m 148$ & 147.9175 & $5.370 E+00$ & day & $4.64 E+05$ & $1.6439 E+05$ & $6.0832 E-06$ & 0.007691 & & $3.312 \mathrm{E}-09$ \\
\hline $\mathrm{Pm} \mathrm{148m}$ & 147.9175 & $4.129 E+01$ & day & $3.57 E+06$ & $2.1380 E+04$ & 4.6774E-05 & 0.012780 & & $8.022 E-09$ \\
\hline Pm 149 & 148.9183 & $5.308 E+01$ & hour & $1.91 E+05$ & $3.9646 E+05$ & $2.5223 E-06$ & & & $1.026 \mathrm{E}-02$ \\
\hline $\mathrm{Pm} \mathrm{150}$ & 149.9210 & $2.680 E+00$ & hour & $9.65 E+03$ & $7.7997 E+06$ & $1.2821 \mathrm{E}-07$ & & & $2.529 E-06$ \\
\hline $\mathrm{Pm} 151$ & 150.9212 & $2.840 E+01$ & hour & $1.02 E+05$ & $7.3115 E+05$ & 1.3677E-06 & & & $4.076 \mathrm{E}-03$ \\
\hline $\mathrm{Pm} 152$ & 151.9235 & $4.120 \mathrm{E}+00$ & minute & $2.47 E+02$ & $3.0040 E+08$ & 3.3289E-09 & & & $5.207 \mathrm{E}-03$ \\
\hline $\mathrm{Pm} \mathrm{152m}$ & 151.9235 & $7.520 E+00$ & minute & $4.51 E+02$ & $1.6458 E+08$ & $6.0760 E-09$ & & & \\
\hline $\mathrm{Pm} \mathrm{152 \textrm {m } 2}$ & 151.9235 & $1.380 E+01$ & minute & $8.28 E+02$ & $8.9685 E+07$ & $1.1150 E-08$ & & & \\
\hline $\mathrm{Pm} 153$ & 152.9241 & $5.400 \mathrm{E}+00$ & minute & $3.24 E+02$ & $2.2770 E+08$ & $4.3918 \mathrm{E}-09$ & & & 1.749E-03 \\
\hline $\mathrm{Pm} 154$ & 153.9265 & $1.730 E+00$ & minute & $1.04 E+02$ & $7.0610 E+08$ & 1.4162E-09 & & & $5.790 E-04$ \\
\hline $\mathrm{Pm}$ 154m & 153.9265 & $2.680 \mathrm{E}+00$ & minute & $1.61 E+02$ & $4.5580 \mathrm{E}+08$ & $2.1939 \mathrm{E}-09$ & & & $1.553 E-04$ \\
\hline Pm 155 & 154.9281 & $4.150 E+01$ & second & $4.15 E+01$ & $1.7547 \mathrm{E}+09$ & $5.6990 E-10$ & & & $5.310 \mathrm{E}-04$ \\
\hline $\mathrm{Pm} 156$ & 155.9311 & $2.670 \mathrm{E}+01$ & second & $2.67 E+01$ & $2.7098 E+09$ & $3.6903 \mathrm{E}-10$ & & & 1.603E-04 \\
\hline $\mathrm{Pm} 157$ & 156.9332 & $1.056 E+01$ & second & $1.06 E+01$ & $6.8077 E+09$ & $1.4689 E-10$ & & & $7.149 \mathrm{E}-05$ \\
\hline $\mathrm{Pm} 158$ & 157.9367 & $4.800 E+00$ & second & $4.80 E+00$ & $1.4882 E+10$ & $6.7197 \mathrm{E}-11$ & & & $2.325 \mathrm{E}-05$ \\
\hline $\operatorname{Sm} 131$ & 130.9358 & $1.200 E+00$ & second & $1.20 \mathrm{E}+00$ & $7.1802 E+10$ & $1.3927 \mathrm{E}-11$ & & & \\
\hline $\operatorname{sm} 132$ & 131.9408 & $4.000 E+00$ & second & $4.00 E+00$ & $2.1377 E+10$ & $4.6780 \mathrm{E}-11$ & & & \\
\hline $\mathrm{Sm} 133$ & 132.9387 & $3.700 \mathrm{E}+00$ & second & $3.70 \mathrm{E}+00$ & $2.2936 E+10$ & $4.3599 E-11$ & & & \\
\hline $\mathrm{Sm} 134$ & 133.9285 & $1.200 E+01$ & second & $1.20 E+01$ & $7.0198 E+09$ & $1.4246 \mathrm{E}-10$ & & & \\
\hline $\mathrm{Sm} 135$ & 134.9324 & $1.000 \mathrm{E}+01$ & second & $1.00 \mathrm{E}+01$ & $8.3610 E+09$ & $1.1960 E-10$ & & & \\
\hline Sm 136 & 135.9283 & $4.700 E+01$ & second & $4.70 E+01$ & $1.7659 E+09$ & $5.6628 E-10$ & & & \\
\hline
\end{tabular}

WSRC-TR-99-00344

October, 1999

page 40 
Radionuclide Specific Data

\begin{tabular}{|c|c|c|c|c|c|c|c|c|c|}
\hline Isotope & $\begin{array}{c}\text { Atomic } \\
\text { Mass }\end{array}$ & Halflife & $\begin{array}{l}\text { Time } \\
\text { Units }\end{array}$ & $t_{1 / 2}, \sec$ & Curies/gram & Grams/ Curie & $\begin{array}{c}\text { Watts/Ci } \\
\text { (Ref.3) }\end{array}$ & $\begin{array}{c}\text { Individual }^{1} \\
\text { Fission } \\
\text { Product Yield } \\
\text { (Ref.4) }\end{array}$ & $\begin{array}{l}\text { Cumulative }^{1} \\
\text { Fission Product } \\
\text { Yield (Ref.4) }\end{array}$ \\
\hline Sm 137 & 136.9270 & $4.500 E+01$ & second & $4.50 E+01$ & $1.8309 \mathrm{E}+09$ & $5.4617 \mathrm{E}-10$ & & & \\
\hline Sm 138 & 137.9235 & $3.100 \mathrm{E}+00$ & minute & $1.86 \mathrm{E}+02$ & $4.3977 \mathrm{E}+08$ & $2.2739 \mathrm{E}-09$ & & & \\
\hline$S \mathrm{Sm} 139$ & 138.9223 & $2.570 \mathrm{E}+00$ & minute & $1.54 E+02$ & $5.2665 \mathrm{E}+08$ & $1.8988 \mathrm{E}-09$ & & & \\
\hline Sm 139m & 138.9223 & $1.070 \mathrm{E}+01$ & second & $1.07 E+01$ & $7.5896 \mathrm{E}+09$ & $1.3176 \mathrm{E}-10$ & & & \\
\hline$S m 140$ & 139.9190 & $1.482 E+01$ & minute & $8.89 E+02$ & $9.0678 \mathrm{E}+07$ & $1.1028 \mathrm{E}-08$ & & & \\
\hline Sm 141 & 140.9185 & $1.020 E+01$ & minute & $6.12 E+02$ & $1.3081 E+08$ & $7.6444 \mathrm{E}-09$ & & & \\
\hline $\mathrm{Sm} 141 \mathrm{~m}$ & 140.9185 & $2.260 E+01$ & minute & $1.36 \mathrm{E}+03$ & $5.9040 E+07$ & $1.6938 \mathrm{E}-08$ & & & \\
\hline$\widehat{S m ~} 142$ & 141.9152 & $7.249 E+01$ & minute & $4.35 E+03$ & $1.8278 \mathrm{E}+07$ & $5.4712 E-08$ & & & \\
\hline $\operatorname{Sm} 143$ & 142.9146 & $8.830 E+00$ & minute & $5.30 \mathrm{E}+02$ & $1.4900 \mathrm{E}+08$ & $6.7114 \mathrm{E}-09$ & & & \\
\hline $\mathrm{Sm} 143 \mathrm{~m}$ & 142.9146 & $1.100 E+00$ & minute & $6.60 E+01$ & $1.1961 E+09$ & $8.3607 \mathrm{E}-10$ & & & \\
\hline $\mathrm{Sm} 143 \mathrm{~m} 2$ & 142.9146 & $3.000 \mathrm{E}-02$ & second & $3.00 E-02$ & $2.6313 E+12$ & $3.8003 E-13$ & & & \\
\hline Sm 145 & 144.9134 & $3.400 E+02$ & day & $2.94 E+07$ & $2.6502 E+03$ & $3.7733 \mathrm{E}-04$ & & & \\
\hline Sm 146 & 145.9130 & $1.030 E+08$ & year & $3.25 E+15$ & $2.3786 \mathrm{E}-05$ & $4.2041 \mathrm{E}+04$ & & & $9.542 \mathrm{E}-13$ \\
\hline Sm 147 & 146.9149 & $1.060 E+11$ & year & $3.35 E+18$ & $2.2956 \mathrm{E}-08$ & $4.3562 \mathrm{E}+07$ & $=$ & & $2.096 \mathrm{E}-02$ \\
\hline Sm 148 & 147.9148 & $7.000 E+15$ & year & $2.21 E+23$ & $3.4526 \mathrm{E}-13$ & $2.8963 E+12$ & & & $1.097 \mathrm{E}-08$ \\
\hline Sm 149 & 148.9172 & $2.000 E+15$ & year & $6.31 E+22$ & $1.2003 \mathrm{E}-12$ & $8.3313 \mathrm{E}+11$ & & $1.392 \mathrm{E}-13$ & $1.608 \mathrm{E}-02$ \\
\hline Sm 151 & 150.9199 & $9.000 E+01$ & year & $2.84 E+09$ & $2.6319 \mathrm{E}+01$ & $3.7995 \mathrm{E}-02$ & 0.000741 & $5.126 \mathrm{E}-10$ & $8.006 \mathrm{E}-03$ \\
\hline Sm 153 & 152.9221 & $4.627 E+01$ & hour & $1.67 E+05$ & $4.4290 \mathrm{E}+05$ & $2.2579 \mathrm{E}-06$ & & $2.513 \mathrm{E}-07$ & $4.109 \mathrm{E}-03$ \\
\hline$\widehat{S m ~ 155}$ & 154.9246 & $2.230 E+01$ & minute & $1.34 E+03$ & $5.4425 \mathrm{E}+07$ & $1.8374 \mathrm{E}-08$ & & $7.889 \mathrm{E}-06$ & $1.327 \mathrm{E}-03$ \\
\hline Sm 156 & 155.9255 & $9.400 E+00$ & hour & $3.38 E+04$ & $2.1381 E+06$ & $4.6770 \mathrm{E}-07$ & & $2.135 \mathrm{E}-05$ & $6.748 \mathrm{E}-04$ \\
\hline Sm 157 & 156.9284 & $4.820 E+02$ & second & $4.82 \mathrm{E}+02$ & $1.4915 \mathrm{E}+08$ & $6.7046 \mathrm{E}-09$ & & $4.447 \mathrm{E}-05$ & $3.869 \mathrm{E}-04$ \\
\hline Sm 158 & 157.9300 & $5.300 E+00$ & minute & $3.18 E+02$ & $2.2464 E+08$ & $4.4516 \mathrm{E}-09$ & & $6.452 \mathrm{E}-05$ & $1.722 \mathrm{E}-04$ \\
\hline Sm 159 & 158.9332 & $1.137 E+01$ & second & $1.14 E+01$ & $6.2431 E+09$ & $1.6018 E-10$ & & $4.787 \mathrm{E}-05$ & $7.863 \mathrm{E}-05$ \\
\hline Sm 160 & 159.9351 & $9.600 \mathrm{E}+00$ & second & $9.60 \mathrm{E}+00$ & $7.3479 \mathrm{E}+09$ & $1.3609 \mathrm{E}-10$ & & $2.533 \mathrm{E}-05$ & $2.971 \mathrm{E}-05$ \\
\hline Eu 136 & 135.9390 & $3.300 E+00$ & second & $3.30 \mathrm{E}+00$ & $2.5149 \mathrm{E}+10$ & $3.9763 \mathrm{E}-11$ & & & \\
\hline Eu $136 \mathrm{~m}$ & 135.9390 & $3.700 E+00$ & second & $3.70 E+00$ & $2.2430 \mathrm{E}+10$ & $4.4583 \mathrm{E}-11$ & & & \\
\hline \begin{tabular}{|l|} 
Eu 137 \\
\end{tabular} & 136.9352 & $1.100 E+01$ & second & $1.10 \mathrm{E}+01$ & $7.4898 \mathrm{E}+09$ & $1.3352 \mathrm{E}-10$ & & & \\
\hline Eu 138 & 137.9335 & $1.210 E+01$ & second & $1.21 E+01$ & $6.7596 \mathrm{E}+09$ & $1.4794 \mathrm{E}-10$ & & & \\
\hline \begin{tabular}{|l} 
Eu 139 \\
\end{tabular} & 138.9298 & $1.790 E+01$ & second & $1.79 \mathrm{E}+01$ & $4.5366 E+09$ & $2.2043 \mathrm{E}-10$ & & & \\
\hline Eu 140 & 139.9281 & $1.510 \mathrm{E}+00$ & second & $1.51 E+00$ & $5.3394 \mathrm{E}+10$ & $1.8729 \mathrm{E}-11$ & & & \\
\hline Eu $140 \mathrm{~m}$ & 139.9281 & $1.250 \mathrm{E}-01$ & second & $1.25 \mathrm{E}-01$ & $6.4500 \mathrm{E}+11$ & $1.5504 \mathrm{E}-12$ & & & \\
\hline Eu 141 & 140.9249 & $4.000 E+01$ & second & $4.00 E+01$ & $2.0014 E+09$ & $4.9966 \mathrm{E}-10$ & & & \\
\hline Eu $141 \mathrm{~m}$ & 140.9249 & $2.700 E+00$ & second & $2.70 E+00$ & $2.9650 E+10$ & $3.3727 \mathrm{E}-11$ & & & \\
\hline Eu 142 & 141.9234 & $2.340 E+00$ & second & $2.34 E+00$ & $3.3971 E+10$ & $2.9437 E-11$ & & & \\
\hline Eu $142 m$ & 141.9234 & $1.220 \mathrm{E}+00$ & minute & $7.32 \mathrm{E}+01$ & $1.0860 E+09$ & $9.2085 \mathrm{E}-10$ & & & \\
\hline
\end{tabular}

WSRC-TR-99-00344

October, 1999

page 41 


\begin{tabular}{|c|c|c|c|c|c|c|c|c|c|}
\hline Isotope & $\begin{array}{l}\text { Atomic } \\
\text { Mass }\end{array}$ & Halflife & $\begin{array}{l}\text { Time } \\
\text { Units }\end{array}$ & $t_{1 / 2}, \sec$ & Curles/ gram & Grams/ Curie & $\begin{array}{c}\text { Watts/Cl } \\
\text { (Ref.3) }\end{array}$ & $\begin{array}{c}\text { Individual }^{4} \\
\text { Fission } \\
\text { Product Yield } \\
\text { (Ref.4) } \\
\end{array}$ & $\begin{array}{l}\text { Cumulative }^{4} \\
\text { Fission Product } \\
\text { Yield (Ref.4) }\end{array}$ \\
\hline Eu 143 & 142.9203 & $2.630 E+00$ & minute & $1.58 \mathrm{E}+02$ & $5.0024 E+08$ & 1.9991E-09 & & & \\
\hline Eu 144 & 143.9188 & $1.020 E+01$ & second & $1.02 E+01$ & $7.6853 E+09$ & $1.3012 \mathrm{E}-10$ & & & \\
\hline Eu 145 & 144.9163 & $5.930 E+00$ & day & $5.12 E+05$ & $1.5195 \mathrm{E}+05$ & $6.5813 \mathrm{E}-06$ & & & \\
\hline Eu 146 & 145.9172 & $4.590 E+00$ & day & $3.97 E+05$ & $1.9496 \mathrm{E}+05$ & $5.1293 \mathrm{E}-06$ & & & \\
\hline Eu 147 & 146.9167 & $2.410 E+01$ & day & $2.08 \mathrm{E}+06$ & $3.6879 E+04$ & $2.7116 E-05$ & & & \\
\hline Eu 148 & 147.9182 & $5.450 E+01$ & day & $4.71 E+06$ & $1.6197 \mathrm{E}+04$ & $6.1738 \mathrm{E}-05$ & & & \\
\hline Eu 149 & 148.9179 & $9.310 E+01$ & day & $8.04 E+06$ & $9.4182 E+03$ & $1.0618 \mathrm{E}-04$ & & & \\
\hline Eu 150 & 149.9197 & $3.690 \mathrm{E}+01$ & year & $1.16 \mathrm{E}+09$ & $6.4621 E+01$ & $1.5475 \mathrm{E}-02$ & & 1.273E-17 & $1.273 E-17$ \\
\hline Eu $150 \mathrm{~m}$ & 149.9197 & $1.280 E+01$ & hour & $4.61 \mathrm{E}+04$ & $1.6331 E+06$ & $6.1234 \mathrm{E}-07$ & & $1.994 \mathrm{E}-18$ & $1.994 \mathrm{E}-18$ \\
\hline Eu 152 & 151.9217 & $1.354 E+01$ & year & $4.27 \mathrm{E}+08$ & $1.7376 \mathrm{E}+02$ & $5.7550 \mathrm{E}-03$ & 0.007646 & $2.650 E-09$ & $5.506 E-09$ \\
\hline Eu $152 \mathrm{~m}$ & 151.9217 & $9.274 E+00$ & hour & $3.34 E+04$ & $2.2243 E+06$ & $4.4959 \mathrm{E}-07$ & & $2.604 E-10$ & $2.604 \mathrm{E}-10$ \\
\hline Eu $152 \mathrm{~m} 2$ & 151.9217 & $9.600 E+01$ & minute & $5.76 E+03$ & $1.2892 \mathrm{E}+07$ & $7.7565 \mathrm{E}-08$ & & $2.857 E-09$ & $2.857 \mathrm{E}-09$ \\
\hline Eu 154 & 153.9230 & $8.593 E+00$ & year & $2.71 E+08$ & $2.7028 E+02$ & $3.6999 \mathrm{E}-03$ & 0.009081 & $1.657 \mathrm{E}-10$ & $3.444 E-10$ \\
\hline Eu $154 \mathrm{~m}$ & 153.9230 & $4.630 E+01$ & minute & $2.78 E+03$ & $2.6384 E+07$ & $3.7902 \mathrm{E}-08$ & & $1.787 \mathrm{E}-10$ & $1.787 \mathrm{E}-10$ \\
\hline Eu 155 & 154.9229 & $4.761 E+00$ & year & $1.50 E+08$ & $4.8466 \mathrm{E}+02$ & $2.0633 \mathrm{E}-03$ & 0.000759 & $5.927 \mathrm{E}-09$ & $1.327 E-03$ \\
\hline Eu 156 & 155.9248 & $1.519 \mathrm{E}+01$ & day & $1.31 E+06$ & $5.5130 E+04$ & $1.8139 \mathrm{E}-05$ & & $3.224 E-08$ & $6.748 E-04$ \\
\hline Eu 157 & 156.9254 & $1.518 \mathrm{E}+01$ & hour & $5.46 \mathrm{E}+04$ & $1.3156 E+06$ & $7.6014 \mathrm{E}-07$ & & $2.483 E-07$ & $3.872 E-04$ \\
\hline Eu 158 & 157.9278 & $4.590 E+01$ & minute & $2.75 E+03$ & $2.5939 \mathrm{E}+07$ & $3.8552 E-08$ & & $7.949 E-07$ & $1.730 \mathrm{E}-04$ \\
\hline Eu 159 & 158.9291 & $1.810 E+01$ & minute & $1.09 E+03$ & $6.5365 \mathrm{E}+07$ & 1.5299E-08 & & $2.263 \mathrm{E}-06$ & $8.089 \mathrm{E}-05$ \\
\hline Eu 160 & 159.9320 & $3.800 \mathrm{E}+01$ & second & $3.80 \mathrm{E}+01$ & $1.8563 \mathrm{E}+09$ & $5.3869 \mathrm{E}-10$ & & $2.483 \mathrm{E}-06$ & $3.219 \mathrm{E}-05$ \\
\hline Eu 161 & 160.9337 & $2.600 \mathrm{E}+01$ & second & $2.60 \mathrm{E}+01$ & $2.6962 E+09$ & $3.7089 E-10$ & & $3.044 E-06$ & $1.256 \mathrm{E}-05$ \\
\hline Eu 162 & 161.9370 & $1.060 E+01$ & second & $1.06 \mathrm{E}+01$ & $6.5724 E+09$ & $1.5215 \mathrm{E}-10$ & & $1.762 \mathrm{E}-06$ & $4.411 \mathrm{E}-06$ \\
\hline Gd 137 & 136.9447 & $7.000 E+00$ & second & $7.00 \mathrm{E}+00$ & $1.1769 \mathrm{E}+10$ & $8.4970 \mathrm{E}-11$ & & & \\
\hline Gd 139 & 138.9381 & $4.900 E+00$ & second & $4.90 \mathrm{E}+00$ & $1.6571 E+10$ & $6.0345 \mathrm{E}-11$ & & & \\
\hline Gd 140 & 139.9339 & $1.580 E+01$ & second & $1.58 \mathrm{E}+01$ & $5.1027 \mathrm{E}+09$ & $1.9598 \mathrm{E}-10$ & & & \\
\hline Gd 141 & 140.9322 & $1.400 E+01$ & second & $1.40 \mathrm{E}+01$ & $5.7179 E+09$ & $1.7489 \mathrm{E}-10$ & & & \\
\hline Gd $141 \mathrm{~m}$ & 140.9322 & $2.450 E+01$ & second & $2.45 E+01$ & $3.2674 E+09$ & $3.0606 \mathrm{E}-10$ & & & \\
\hline Gd 142 & 141.9282 & $7.020 E+01$ & second & $7.02 E+01$ & $1.1323 E+09$ & $8.8314 \mathrm{E}-10$ & & & \\
\hline Gd 143 & 142.9267 & $3.900 E+01$ & second & $3.90 \mathrm{E}+01$ & $2.0239 E+09$ & $4.9408 \mathrm{E}-10$ & & & \\
\hline Gd $143 \mathrm{~m}$ & 142.9267 & $1.120 \mathrm{E}+02$ & second & $1.12 \mathrm{E}+02$ & $7.0477 \mathrm{E}+08$ & 1.4189E-09 & & & \\
\hline Gd 144 & 143.9228 & $4.500 \mathrm{E}+00$ & minute & $2.70 E+02$ & $2.9032 E+08$ & $3.4444 E-09$ & & & \\
\hline Gd 145 & 144.9217 & $2.300 E+01$ & minute & $1.38 \mathrm{E}+03$ & $5.6411 \mathrm{E}+07$ & $1.7727 \mathrm{E}-08$ & & & \\
\hline Gd $145 \mathrm{~m}$ & 144.9217 & $8.500 E+01$ & second & $8.50 \mathrm{E}+01$ & $9.1585 \mathrm{E}+08$ & 1.0919E-09 & & & \\
\hline Gd 146 & 145.9183 & $4.827 \mathrm{E}+01$ & day & $4.17 E+06$ & $1.8539 \mathrm{E}+04$ & $5.3942 \mathrm{E}-05$ & & & \\
\hline Gd 147 & 146.9191 & $3.806 \mathrm{E}+01$ & hour & $1.37 \mathrm{E}+05$ & $5.6044 \mathrm{E}+05$ & $1.7843 \mathrm{E}-06$ & & & \\
\hline
\end{tabular}

WSRC-TR-99-00344

October, 1999

page 42 


\begin{tabular}{|c|c|c|c|c|c|c|c|c|c|}
\hline Isotope & $\begin{array}{l}\text { Atomic } \\
\text { Mass }\end{array}$ & Halflife & $\begin{array}{l}\text { Time } \\
\text { Units }\end{array}$ & $t_{112}, \sec$ & Curies/ gram & Grams/ Curie & $\begin{array}{c}\text { Watts/Ci } \\
\text { (Ref.3) }\end{array}$ & \begin{tabular}{|c|} 
Individual $^{1}$ \\
Fission \\
Product Yield \\
(Ref.4) \\
\end{tabular} & $\begin{array}{l}\text { Cumulative }^{4} \\
\text { Fission Product } \\
\text { Yield (Ref.4) }\end{array}$ \\
\hline Gd 148 & 147.9181 & $7.460 \mathrm{E}+01$ & year & $2.35 E+09$ & $3.2397 \mathrm{E}+01$ & $3.0867 \mathrm{E}-02$ & & & \\
\hline Gd 149 & 148.9193 & $9.280 \mathrm{E}+00$ & day & $8.02 E+05$ & $9.4485 E+04$ & $1.0584 \mathrm{E}-05$ & & & \\
\hline Gd 150 & 149.9187 & $1.790 E+06$ & year & $5.65 \mathrm{E}+13$ & $1.3321 \mathrm{E}-03$ & $7.5067 \mathrm{E}+02$ & & & \\
\hline Gd 151 & 150.9203 & $1.240 \mathrm{E}+02$ & day & $1.07 E+07$ & $6.9774 \mathrm{E}+03$ & $1.4332 E-04$ & & $2.013 \mathrm{E}-21$ & $2.013 \mathrm{E}-21$ \\
\hline Gd 152 & 151.9198 & $1.080 \mathrm{E}+14$ & year & $3.41 \mathrm{E}+21$ & $2.1788 \mathrm{E}-11$ & $4.5896 \mathrm{E}+10$ & & $5.226 \mathrm{E}-13$ & $1.685 \mathrm{E}-09$ \\
\hline Gd 153 & 152.9217 & $2.416 \mathrm{E}+02$ & day & $2.09 \mathrm{E}+07$ & $3.5342 \mathrm{E}+03$ & $2.8295 \mathrm{E}-04$ & 0.000838 & $1.415 \mathrm{E}-16$ & $1.415 \mathrm{E}-16$ \\
\hline Gd $155 \mathrm{~m}$ & 154.9226 & 3.197E-02 & second & $3.20 \mathrm{E}-02$ & $2.2778 \mathrm{E}+12$ & $4.3902 \mathrm{E}-13$ & & & \\
\hline Gd 159 & 158.9264 & $1.848 E+01$ & hour & $6.65 E+04$ & $1.0671 \mathrm{E}+06$ & $9.3713 \mathrm{E}-07$ & & $1.922 \mathrm{E}-08$ & $8.091 E-05$ \\
\hline Gd 161 & 160.9297 & $3.660 \mathrm{E}+00$ & minute & $2.20 E+02$ & $3.1923 \mathrm{E}+08$ & $3.1325 \mathrm{E}-09$ & & 2.363E-07 & $1.280 \mathrm{E}-05$ \\
\hline Gd 162 & 161.9310 & $8.400 E+00$ & minute & $5.04 \mathrm{E}+02$ & $1.3823 \mathrm{E}+08$ & $7.2341 \mathrm{E}-09$ & & $5.136 \mathrm{E}-07$ & $4.925 \mathrm{E}-06$ \\
\hline Gd 163 & 162.9340 & $6.800 E+01$ & second & $6.80 \mathrm{E}+01$ & $1.0183 E+09$ & $9.8207 \mathrm{E}-10$ & & 3.544E-07 & $1.203 E-06$ \\
\hline Gd 164 & 163.9359 & $4.500 \mathrm{E}+01$ & second & $4.50 E+01$ & $1.5293 \mathrm{E}+09$ & $6.5390 \mathrm{E}-10$ & & $2.433 \mathrm{E}-07$ & $3.843 E-07$ \\
\hline Tb 140 & 139.9455 & $2.400 \mathrm{E}+00$ & second & $2.40 \mathrm{E}+00$ & $3.3590 \mathrm{E}+10$ & $2.9771 \mathrm{E}-11$ & & & \\
\hline Tb 141 & 140.9412 & $3.500 E+00$ & second & $3.50 E+00$ & $2.2870 E+10$ & $4.3725 \mathrm{E}-11$ & & & \\
\hline Tb 142 & 141.9389 & $5.970 E-01$ & second & $5.97 \mathrm{E}-01$ & $1.3314 E+11$ & $7.5110 \mathrm{E}-12$ & & & \\
\hline Tb $142 \mathrm{~m}$ & 141.9389 & $3.030 \mathrm{E}-01$ & second & $3.03 E-01$ & $2.6232 E+11$ & $3.8121 \mathrm{E}-12$ & & & \\
\hline $\mathrm{Tb} 143$ & 142.9348 & $1.200 \mathrm{E}+01$ & second & $1.20 \mathrm{E}+01$ & $6.5774 \mathrm{E}+09$ & $1.5203 \mathrm{E}-10$ & & & \\
\hline Tb 143m & 142.9348 & $2.100 \mathrm{E}+01$ & second & $2.10 \mathrm{E}+01$ & $3.7585 \mathrm{E}+09$ & $2.6606 \mathrm{E}-10$ & & & \\
\hline Tb 144 & 143.9325 & $1.000 \mathrm{E}+00$ & second & $1.00 \mathrm{E}+00$ & $7.8382 E+10$ & 1.2758E-11 & & & \\
\hline Tb 144m & 143.9325 & $4.250 \mathrm{E}+00$ & second & $4.25 \mathrm{E}+00$ & $1.8443 E+10$ & $5.4221 \mathrm{E}-11$ & & & \\
\hline $\mathrm{Tb} 145 \mathrm{~m}$ & 144.9289 & $2.950 \mathrm{E}+01$ & second & $2.95 \mathrm{E}+01$ & $2.6388 E+09$ & $3.7897 \mathrm{E}-10$ & & & \\
\hline Tb 146 & 145.9272 & $8.000 \mathrm{E}+00$ & second & $8.00 E+00$ & $9.6639 \mathrm{E}+09$ & $1.0348 \mathrm{E}-10$ & & & \\
\hline To $146 \mathrm{~m}$ & 145.9272 & $2.410 E+01$ & second & $2.41 E+01$ & $3.2079 \mathrm{E}+09$ & $3.1173 \mathrm{E}-10$ & & & \\
\hline $\mathrm{Tb} 146 \mathrm{~m} 2$ & 145.9272 & $1.180 \mathrm{E}-03$ & second & $1.18 \mathrm{E}-03$ & $6.5518 \mathrm{E}+13$ & $1.5263 \mathrm{E}-14$ & & & \\
\hline Tb 147 & 146.9240 & $1.700 \mathrm{E}+00$ & hour & $6.12 \mathrm{E}+03$ & $1.2547 \mathrm{E}+07$ & $7.9702 E-08$ & & & \\
\hline Tb 147m & 146.9240 & $1.830 E+00$ & minute & $1.10 E+02$ & $6.9933 E+08$ & $1.4299 \mathrm{E}-09$ & & & \\
\hline Tb 148 & 147.9243 & $1.000 \mathrm{E}+00$ & hour & $3.60 E+03$ & $2.1185 E+07$ & $4.7203 \mathrm{E}-08$ & & & \\
\hline Tb $148 \mathrm{~m}$ & 147.9243 & $2.200 \mathrm{E}+00$ & minute & $1.32 \mathrm{E}+02$ & $5.7778 \mathrm{E}+08$ & $1.7308 \mathrm{E}-09$ & & & \\
\hline Tb 149 & 148.9232 & $4.118 \mathrm{E}+00$ & hour & $1.48 E+04$ & $5.1101 E+06$ & $1.9569 \mathrm{E}-07$ & & & \\
\hline $\mathrm{Tb} 149 \mathrm{~m}$ & 148.9232 & $4.160 \mathrm{E}+00$ & minute & $2.50 E+02$ & $3.0351 E+08$ & $3.2948 \mathrm{E}-09$ & & & \\
\hline Tb 150 & 149.9237 & $3.480 \mathrm{E}+00$ & hour & $1.25 \mathrm{E}+04$ & $6.0065 E+06$ & $1.6649 \mathrm{E}-07$ & & & \\
\hline $\mathrm{Tb} 150 \mathrm{~m}$ & 149.9237 & $5.800 \mathrm{E}+00$ & minute & $3.48 \mathrm{E}+02$ & $2.1624 E+08$ & $4.6246 E-09$ & & & \\
\hline Tb 151 & 150.9231 & $1.761 \mathrm{E}+01$ & hour & $6.34 E+04$ & $1.1792 E+06$ & $5.4333 \mathrm{E}+05$ & & & \\
\hline $\mathrm{Tb} 151 \mathrm{~m}$ & 150.9231 & $2.500 E+01$ & second & $2.50 \mathrm{E}+01$ & $2.9901 E+09$ & $1.5092 \mathrm{E}+02$ & & & \\
\hline Tb 152 & 151.9241 & $1.750 E+01$ & hour & $6.30 E+04$ & $1.1787 \mathrm{E}+06$ & $8.4838 \mathrm{E}-07$ & & & \\
\hline
\end{tabular}




\begin{tabular}{|c|c|c|c|c|c|c|c|c|c|}
\hline Isotope & $\begin{array}{l}\text { Atomic } \\
\text { Mass }\end{array}$ & Halflife & $\begin{array}{l}\text { Time } \\
\text { Units }\end{array}$ & $\mathbf{t}_{122}, \mathrm{sec}$ & Curies/gram & Grams/ Curie & $\begin{array}{c}\text { Watts/Ci } \\
\text { (Ref.3) }\end{array}$ & $\begin{array}{c}\text { Individual }^{1} \\
\text { Fission } \\
\text { Product Yield } \\
\text { (Ref.4) }\end{array}$ & $\begin{array}{c}\text { Cumulative }^{1} \\
\text { Fission Product } \\
\text { Yield (Ref.4) }\end{array}$ \\
\hline Tb 152m & 151.9241 & $4.200 E+00$ & minute & $2.52 E+02$ & $2.9468 \mathrm{E}+08$ & $3.3935 \mathrm{E}-09$ & & & \\
\hline Tb 153 & 152.9234 & $2.340 E+00$ & day & $2.02 \mathrm{E}+05$ & $3.6490 E+05$ & $2.7405 \mathrm{E}-06$ & & & \\
\hline Tb 154 & 153.9247 & $2.150 \mathrm{E}+01$ & hour & $7.74 E+04$ & $9.4695 E+05$ & $1.0560 \mathrm{E}-06$ & & & \\
\hline Tb 154m & 153.9247 & $2.270 \mathrm{E}+01$ & hour & $8.17 E+04$ & $8.9689 E+05$ & $1.1150 \mathrm{E}-06$ & & & \\
\hline Tb 154m2 & 153.9247 & $9.400 E+00$ & hour & $3.38 E+04$ & $2.1659 E+06$ & $4.6170 \mathrm{E}-07$ & & & \\
\hline Tb 155 & 154.9235 & $5.320 E+00$ & day & $4.60 E+05$ & $1.5843 E+05$ & $6.3120 \mathrm{E}-06$ & & & \\
\hline Tb 156 & 155.9247 & $5.350 E+00$ & day & $4.62 E+05$ & $1.5653 E+05$ & $6.3886 \mathrm{E}-06$ & & & \\
\hline Tb $156 \mathrm{~m}$ & 155.9247 & $2.440 E+01$ & hour & $8.78 E+04$ & $8.2370 E+05$ & $1.2140 \mathrm{E}-06$ & & & \\
\hline Tb $156 \mathrm{~m} 2$ & 155.9247 & $5.000 E+00$ & hour & $1.80 E+04$ & $4.0197 E+06$ & $2.4878 \mathrm{E}-07$ & & & \\
\hline Tb 157 & 156.9240 & $7.100 E+01$ & year & $2.24 E+09$ & $3.2086 E+01$ & $3.1166 \mathrm{E}-02$ & 0.000048 & $2.068 \mathrm{E}-15$ & $2.068 \mathrm{E}-15$ \\
\hline Tb 158 & 157.9254 & $1.800 E+02$ & year & $5.68 \mathrm{E}+09$ & $1.2576 E+01$ & $7.9518 \mathrm{E}-02$ & 0.005347 & $7.502 \mathrm{E}-14$ & $8.240 \mathrm{E}-14$ \\
\hline Tb $158 \mathrm{~m}$ & 157.9254 & $1.070 E+01$ & second & $1.07 E+01$ & $6.6764 \mathrm{E}+09$ & $1.4978 \mathrm{E}-10$ & & $7.374 \mathrm{E}-15$ & $7.374 \mathrm{E}-15$ \\
\hline Tb 160 & 159.9272 & $7.230 E+01$ & day & $6.25 E+06$ & $1.1293 E+04$ & $8.8552 E-05$ & 0.008186 & $3.024 \mathrm{E}-11$ & $3.024 \mathrm{E}-11$ \\
\hline Tb 161 & 160.9276 & $6.880 E+00$ & day & $5.94 E+05$ & $1.1794 E+05$ & $8.4792 \mathrm{E}-06$ & & $3.524 \mathrm{E}-10$ & $1.280 \mathrm{E}-05$ \\
\hline Tb 162 & 161.9295 & $7.600 E+00$ & minute & $4.56 E+02$ & $1.5279 E+08$ & $6.5451 \mathrm{E}-09$ & & $1.814 \mathrm{E}-09$ & $4.926 \mathrm{E}-06$ \\
\hline Tb 163 & 162.9306 & $1.950 E+01$ & minute & $1.17 \mathrm{E}+03$ & $5.9182 E+07$ & $1.6897 \mathrm{E}-08$ & & $5.046 \mathrm{E}-09$ & $1.208 \mathrm{E}-06$ \\
\hline Tb $163 \mathrm{~m}$ & 162.9306 & $6.500 E+00$ & hour & $2.34 E+04$ & $2.9591 E+06$ & $3.3794 \mathrm{E}-07$ & & & \\
\hline Tb $163 \mathrm{~m} 2$ & 162.9306 & $7.100 E+00$ & minute & $4.26 E+02$ & $1.6254 E+08$ & $6.1523 \mathrm{E}-09$ & & & \\
\hline Tb 164 & 163.9333 & $3.000 E+00$ & minute & $1.80 E+02$ & $3.8233 E+08$ & $2.6156 \mathrm{E}-09$ & & $7.579 E-09$ & $3.919 \mathrm{E}-07$ \\
\hline Tb 165 & 164.9349 & $2.110 E+00$ & minute & $1.27 E+02$ & $5.4029 E+08$ & $1.8508 \mathrm{E}-09$ & & $1.261 \mathrm{E}-08$ & $1.500 \mathrm{E}-07$ \\
\hline Dy 141 & 140.9512 & $9.000 \mathrm{E}-01$ & second & $9.00 \mathrm{E}-01$ & $8.8933 E+10$ & $1.1244 \mathrm{E}-11$ & & & \\
\hline Dy 142 & 141.9463 & $2.300 E+00$ & second & $2.30 E+00$ & $3.4556 \mathrm{E}+10$ & $2.8938 \mathrm{E}-11$ & & & \\
\hline Dy 143 & 142.9438 & $4.100 \mathrm{E}+00$ & second & $4.10 E+00$ & $1.9250 E+10$ & $5.1948 \mathrm{E}-11$ & & & \\
\hline Dy 144 & 143.9391 & $9.100 \mathrm{E}+00$ & second & $9.10 E+00$ & $8.6130 E+09$ & $1.1610 \mathrm{E}-10$ & & & \\
\hline Dy 145 & 144.9370 & $1.000 \mathrm{E}+01$ & second & $1.00 \mathrm{E}+01$ & $7.7839 \mathrm{E}+09$ & $1.2847 E-10$ & & & \\
\hline \begin{tabular}{|l} 
Dy $145 m$ \\
\end{tabular} & 144.9370 & $1.360 E+01$ & second & $1.36 \mathrm{E}+01$ & $5.7235 E+09$ & $1.7472 \mathrm{E}-10$ & & & \\
\hline Dy 146 & 145.9327 & $2.900 \mathrm{E}+01$ & second & $2.90 \mathrm{E}+01$ & $2.6658 \mathrm{E}+09$ & $3.7512 \mathrm{E}-10$ & & & \\
\hline Dy $146 \mathrm{~m}$ & 145.9327 & $1.500 \mathrm{E}-01$ & second & $1.50 \mathrm{E}-01$ & $5.1539 \mathrm{E}+11$ & $1.9403 \mathrm{E}-12$ & & & \\
\hline Dy 147 & 146.9309 & $4.000 \mathrm{E}+01$ & second & $4.00 E+01$ & $1.9196 \mathrm{E}+09$ & $5.2095 \mathrm{E}-10$ & & & \\
\hline Dy $147 \mathrm{~m}$ & 146.9309 & $5.570 \mathrm{E}+01$ & second & $5.57 E+01$ & $1.3785 E+09$ & $7.2542 \mathrm{E}-10$ & & & \\
\hline Dy 148 & 147.9272 & $3.100 E+00$ & minute & $1.86 E+02$ & $4.1003 E+08$ & $2.4388 \mathrm{E}-09$ & & & \\
\hline Dy 149 & 148.9273 & $4.200 E+00$ & minute & $2.52 \mathrm{E}+02$ & $3.0061 E+08$ & $3.3266 \mathrm{E}-09$ & & & \\
\hline Dy $149 m$ & 148.9273 & $4.900 \mathrm{E}-01$ & second & $4.90 \mathrm{E}-01$ & $1.5460 \mathrm{E}+11$ & $6.4684 \mathrm{E}-12$ & & & \\
\hline Dy 150 & 149.9256 & $7.170 E+00$ & minute & $4.30 E+02$ & $1.7492 E+08$ & 5.7170 E-09 & & & \\
\hline Dy 151 & 150.9262 & $1.790 \mathrm{E}+01$ & minute & $1.07 E+03$ & $6.9600 \mathrm{E}+07$ & $1.4368 \mathrm{E}-08$ & & & \\
\hline
\end{tabular}

WSRC-TR-99-00344

October, 1999

page 44 


\begin{tabular}{|c|c|c|c|c|c|c|c|c|c|}
\hline Isotope & $\begin{array}{l}\text { Atomic } \\
\text { Mass }\end{array}$ & Halflife & $\begin{array}{l}\text { Time } \\
\text { Units }\end{array}$ & $t_{1 / 2}, \sec$ & Curies/ gram & Grams/ Curie & $\begin{array}{c}\text { Watts/Ci } \\
\text { (Ref.3) }\end{array}$ & $\begin{array}{c}\text { Individual }^{1} \\
\text { Fission }^{-} \\
\text {Product Yield } \\
\text { (Ref.4) }^{\text {Red }}\end{array}$ & $\begin{array}{l}\text { Cumulative }^{4} \\
\text { Fission Product } \\
\text { Yield (Ref.4) }\end{array}$ \\
\hline Dy 152 & 151.9247 & $2.380 E+00$ & hour & $8.57 \mathrm{E}+03$ & $8.6670 \mathrm{E}+06$ & $1.1538 \mathrm{E}-07$ & & & \\
\hline Dy 153 & 152.9258 & $6.400 E+00$ & hour & $2.30 E+04$ & $3.2019 \mathrm{E}+06$ & $3.1231 \mathrm{E}-07$ & & & \\
\hline Dy 154 & 153.9244 & $3.000 E+06$ & year & $9.47 \mathrm{E}+13$ & $7.7416 \mathrm{E}-04$ & $1.2917 \mathrm{E}+03$ & & & \\
\hline Dy 155 & 154.9257 & $9.900 \mathrm{E}+00$ & hour & $3.56 \mathrm{E}+04$ & $2.0432 E+06$ & $4.8942 \mathrm{E}-07$ & & & \\
\hline Dy 157 & 156.9255 & $8.140 E+00$ & hour & $2.93 E+04$ & $2.4533 E+06$ & $4.0761 \mathrm{E}-07$ & & $3.303 E-21$ & $3.303 \mathrm{E}-21$ \\
\hline Dy $157 \mathrm{~m}$ & 156.9255 & $2.160 \mathrm{E}-02$ & second & $2.16 \mathrm{E}-02$ & $3.3284 \mathrm{E}+12$ & $3.0045 \mathrm{E}-13$ & & & \\
\hline Dy 159 & 158.9257 & $1.444 E+02$ & day & $1.25 E+07$ & $5.6899 \mathrm{E}+03$ & $1.7575 \mathrm{E}-04$ & & $3.859 \mathrm{E}-17$ & $3.859 \mathrm{E}-17$ \\
\hline Dy 165 & 164.9317 & $2.334 \mathrm{E}+00$ & hour & $8.40 E+03$ & $8.1408 \mathrm{E}+06$ & $1.2284 \mathrm{E}-07$ & & $2.520 E-10$ & $1.478 \mathrm{E}-07$ \\
\hline Dy $165 \mathrm{~m}$ & 164.9317 & $1.257 \mathrm{E}+00$ & minute & $7.54 \mathrm{E}+01$ & $9.0696 \mathrm{E}+08$ & $1.1026 \mathrm{E}-09$ & & $3.355 E-11$ & $1.095 \mathrm{E}-07$ \\
\hline Dy 166 & 165.9328 & $8.160 \mathrm{E}+01$ & hour & $2.94 E+05$ & $2.3145 \mathrm{E}+05$ & $4.3206 \mathrm{E}-06$ & & $8.440 \mathrm{E}-10$ & $5.384 \mathrm{E}-08$ \\
\hline Dy 167 & 166.9356 & $6.200 E+00$ & minute & $3.72 E+02$ & $1.8167 \mathrm{E}+08$ & $5.5045 \mathrm{E}-09$ & & $1.241 \mathrm{E}-09$ & $1.596 \mathrm{E}-08$ \\
\hline Dy 168 & 167.9372 & $8.700 E+00$ & minute & $5.22 \mathrm{E}+02$ & $1.2869 \mathrm{E}+08$ & $7.7704 \mathrm{E}-09$ & & $2.032 \mathrm{E}-09$ & $6.803 E-09$ \\
\hline Dy 169 & 168.9403 & $3.900 \mathrm{E}+01$ & second & $3.90 \mathrm{E}+01$ & $1.7123 E+09$ & $5.8401 \mathrm{E}-10$ & & 1.191E-09 & $2.203 \mathrm{E}-09$ \\
\hline Ho 144 & 143.9516 & $7.000 E-01$ & second & $7.00 \mathrm{E}-01$ & $1.1196 \mathrm{E}+11$ & $8.9318 \mathrm{E}-12$ & & & \\
\hline Ho 145 & 144.9469 & $2.400 \mathrm{E}+00$ & second & $2.40 \mathrm{E}+00$ & $3.2431 E+10$ & $3.0835 \mathrm{E}-11$ & & & \\
\hline Ho 146 & 145.9441 & $3.600 \mathrm{E}+00$ & second & $3.60 \mathrm{E}+00$ & $2.1473 \mathrm{E}+10$ & $4.6571 \mathrm{E}-11$ & & & \\
\hline Ho 147 & 146.9398 & $5.800 E+00$ & second & $5.80 \mathrm{E}+00$ & $1.3238 \mathrm{E}+10$ & $7.5542 \mathrm{E}-11$ & & & \\
\hline Ho 148 & 147.9373 & $2.200 E+00$ & second & $2.20 E+00$ & $3.4664 E+10$ & $2.8849 \mathrm{E}-11$ & & & \\
\hline Ho $148 \mathrm{~m}$ & 147.9373 & $9.300 E+00$ & second & $9.30 \mathrm{E}+00$ & $8.2000 E+09$ & $1.2195 \mathrm{E}-10$ & & & \\
\hline Ho 149 & 148.9338 & $2.110 E+01$ & second & $2.11 E+01$ & $3.5901 E+09$ & $2.7855 \mathrm{E}-10$ & & & \\
\hline Ho $149 \mathrm{~m}$ & 148.9338 & $5.600 E+01$ & second & $5.60 E+01$ & $1.3527 E+09$ & $7.3927 \mathrm{E}-10$ & & & \\
\hline Ho 150 & 149.9334 & $7.200 E+01$ & second & $7.20 \mathrm{E}+01$ & $1.0451 E+09$ & $9.5687 \mathrm{E}-10$ & & & \\
\hline Ho $150 \mathrm{~m}$ & 149.9334 & $2.600 \mathrm{E}+01$ & second & $2.60 E+01$ & $2.8940 E+09$ & $3.4554 \mathrm{E}-10$ & & & \\
\hline Ho 151 & 150.9317 & $3.520 \mathrm{E}+01$ & second & $3.52 E+01$ & $2.1235 E+09$ & $4.7092 \mathrm{E}-10$ & & & \\
\hline Ho 151m & 150.9317 & $4.720 E+01$ & second & $4.72 E+01$ & $1.5836 \mathrm{E}+09$ & $6.3146 \mathrm{E}-10$ & & & \\
\hline Ho 152 & 151.9317 & $1.618 E+02$ & second & $1.62 E+02$ & $4.5893 E+08$ & $2.1790 E-09$ & & & \\
\hline Ho.152m & 151.9317 & $5.000 E+01$ & second & $5.00 E+01$ & $1.4851 \mathrm{E}+09$ & $6.7335 \mathrm{E}-10$ & & & \\
\hline Ho 153 & 152.9302 & $2.020 \mathrm{E}+00$ & minute & $1.21 E+02$ & $6.0867 E+08$ & $1.6429 \mathrm{E}-09$ & & & \\
\hline Ho $153 \mathrm{~m}$ & 152.9302 & $9.300 \mathrm{E}+00$ & minute & $5.58 \mathrm{E}+02$ & $1.3221 \mathrm{E}+08$ & $7.5640 \mathrm{E}-09$ & & & \\
\hline Ho 154 & 153.9306 & $1.176 \mathrm{E}+01$ & minute & $7.06 \mathrm{E}+02$ & $1.0387 E+08$ & $9.6274 E-09$ & & & \\
\hline Ho $154 \mathrm{~m}$ & 153.9306 & $3.100 E+00$ & minute & $1.86 \mathrm{E}+02$ & $3.9404 E+08$ & $2.5378 E-09$ & & & \\
\hline Ho 155 & 154.9291 & $4.800 E+01$ & minute & $2.88 \mathrm{E}+03$ & $2.5284 E+07$ & $3.9550 \mathrm{E}-08$ & & & \\
\hline Ho 156 & 155.9297 & $5.600 \mathrm{E}+01$ & minute & $3.36 \mathrm{E}+03$ & $2.1533 E+07$ & $4.6440 \mathrm{E}-08$ & & & \\
\hline Ho 157 & 156.9282 & $1.260 E+01$ & minute & $7.56 \mathrm{E}+02$ & $9.5094 \mathrm{E}+07$ & $1.0516 \mathrm{E}-08$ & & & \\
\hline Ho 158 & 157.9289 & $1.130 \mathrm{E}+01$ & minute & $6.78 \mathrm{E}+02$ & $1.0536 \mathrm{E}+08$ & $9.4911 \mathrm{E}-09$ & & & \\
\hline
\end{tabular}




\begin{tabular}{|c|c|c|c|c|c|c|c|c|c|}
\hline Isotope & $\begin{array}{l}\text { Atomic } \\
\text { Mass }\end{array}$ & Halflife & $\begin{array}{l}\text { Time } \\
\text { Units }\end{array}$ & $t_{1 / 2}, \mathrm{sec}$ & Curies/ gram & Grams/ Curie & $\begin{array}{c}\text { Watts/CI } \\
\text { (Ref.3) }\end{array}$ & $\begin{array}{c}\text { Individual }^{1} \\
\text { Fission } \\
\text { Product Yield } \\
\text { (Ref.4) }\end{array}$ & $\begin{array}{l}\text { Cumulative } \\
\text { Fission Product } \\
\text { Yield (Ref.4) }\end{array}$ \\
\hline Ho $158 \mathrm{~m}$ & 157.9289 & $2.800 E+01$ & minute & $1.68 \mathrm{E}+03$ & $4.2521 E+07$ & $2.3518 E-08$ & & & \\
\hline Ho $158 \mathrm{~m} 2$ & 157.9289 & $2.130 \mathrm{E}+01$ & minute & $1.28 \mathrm{E}+03$ & $5.5896 \mathrm{E}+07$ & 1.7890 E-08 & & & \\
\hline Ho 159 & 158.9277 & $3.305 E+01$ & minute & $1.98 E+03$ & $3.5798 E+07$ & $2.7935 E-08$ & & & \\
\hline Ho $159 \mathrm{~m}$ & 158.9277 & $8.300 \mathrm{E}+00$ & second & $8.30 E+00$ & $8.5526 E+09$ & $1.1692 E-10$ & & & \\
\hline Ho 160 & 159.9287 & $2.560 E+01$ & minute & $1.54 \mathrm{E}+03$ & $4.5926 E+07$ & $2.1774 E-08$ & & & \\
\hline Ho $160 \mathrm{~m}$ & 159.9287 & $5.020 \mathrm{E}+00$ & hour & $1.81 E+04$ & $3.9034 \mathrm{E}+06$ & $2.5619 E-07$ & & & \\
\hline Ho $160 \mathrm{~m} 2$ & 159.9287 & $3.000 \mathrm{E}+00$ & second & $3.00 E+00$ & $2.3514 E+10$ & $4.2528 \mathrm{E}-11$ & & & \\
\hline Ho 161 & 160.9279 & $2.480 \mathrm{E}+00$ & hour & $8.93 E+03$ & $7.8522 \mathrm{E}+06$ & 1.2735E-07 & & & \\
\hline Ho $161 \mathrm{~m}$ & 160.9279 & $6.760 E+00$ & second & $6.76 \mathrm{E}+00$ & $1.0370 E+10$ & $9.6428 \mathrm{E}-11$ & & & \\
\hline Ho 162 & 161.9291 & $1.500 \mathrm{E}+01$ & minute & $9.00 \mathrm{E}+02$ & $7.7412 \mathrm{E}+07$ & $1.2918 \mathrm{E}-08$ & & & \\
\hline Ho $162 \mathrm{~m}$ & 161.9291 & $6.700 \mathrm{E}+01$ & minute & $4.02 E+03$ & $1.7331 E+07$ & $5.7700 \mathrm{E}-08$ & & & \\
\hline Ho 163 & 162.9287 & $4.570 E+03$ & year & $1.44 E+11$ & $4.8012 \mathrm{E}-01$ & $2.0828 \mathrm{E}+00$ & & & \\
\hline Ho $163 \mathrm{~m}$ & 162.9287 & $1.090 \mathrm{E}+00$ & second & $1.09 \mathrm{E}+00$ & $6.3526 E+10$ & $1.5742 E-11$ & & & \\
\hline Ho 164 & 163.9302 & $2.900 E+01$ & minute & $1.74 E+03$ & $3.9552 E+07$ & $2.5283 \mathrm{E}-08$ & & & \\
\hline Ho $164 \mathrm{~m}$ & 163.9302 & $3.750 \mathrm{E}+01$ & minute & $2.25 \mathrm{E}+03$ & $3.0587 E+07$ & $3.2694 \mathrm{E}-08$ & & & \\
\hline Ho 166 & 165.9323 & $2.683 E+01$ & hour & $9.66 \mathrm{E}+04$ & $7.0392 E+05$ & $1.4206 \mathrm{E}-06$ & & & \\
\hline Ho $166 \mathrm{~m}$ & 165.9323 & $1.200 E+03$ & year & $3.79 \mathrm{E}+10$ & $1.7953 E+00$ & $5.5700 \mathrm{E}-01$ & & & \\
\hline Ho 167 & 166.9331 & $3.100 E+00$ & hour & $1.12 \mathrm{E}+04$ & $6.0558 E+06$ & $1.6513 \mathrm{E}-07$ & & & \\
\hline Ho 168 & 167.9355 & $2.990 E+00$ & minute & $1.79 \mathrm{E}+02$ & $3.7447 E+08$ & $2.6705 \mathrm{E}-09$ & & & \\
\hline Ho $168 \mathrm{~m}$ & 167.9355 & $1.320 \mathrm{E}+02$ & second & $1.32 \mathrm{E}+02$ & $5.0893 E+08$ & $1.9649 \mathrm{E}-09$ & & & \\
\hline Ho 169 & 168.9369 & $4.700 \mathrm{E}+00$ & minute & $2.82 E+02$ & $2.3681 \mathrm{E}+08$ & $4.2228 E-09$ & & & \\
\hline Ho 170 & 169.9396 & $4.300 E+01$ & second & $4.30 \mathrm{E}+01$ & $1.5439 E+09$ & $6.4772 \mathrm{E}-10$ & & & \\
\hline Ho $170 \mathrm{~m}$ & 169.9396 & $2.800 \mathrm{E}+00$ & minute & $1.68 \mathrm{E}+02$ & $3.9516 E+08$ & 2.5306 E-09 & & & \\
\hline Ho 171 & 170.9415 & $5.300 E+01$ & second & $5.30 \mathrm{E}+01$ & $1.2452 E+09$ & $8.0306 E-10$ & & & \\
\hline Ho 172 & 171.9448 & $2.500 \mathrm{E}+01$ & second & $2.50 \mathrm{E}+01$ & $2.6245 E+09$ & $3.8102 \mathrm{E}-10$ & & & \\
\hline Er 145 & 144.9575 & $9.000 \mathrm{E}-01$ & second & $9.00 \mathrm{E}-01$ & $8.6476 E+10$ & $1.1564 \mathrm{E}-11$ & & & \\
\hline Er 147 & 146.9493 & $2.500 \mathrm{E}+00$ & second & $2.50 \mathrm{E}+00$ & $3.0709 E+10$ & $3.2563 \mathrm{E}-11$ & & & \\
\hline Er $147 \mathrm{~m}$ & 146.9493 & $2.500 \mathrm{E}+00$ & second & $2.50 \mathrm{E}+00$ & $3.0709 E+10$ & $3.2563 \mathrm{E}-11$ & & & \\
\hline Er 148 & 147.9444 & $4.600 E+00$ & second & $4.60 \mathrm{E}+00$ & $1.6578 E+10$ & $6.0323 \mathrm{E}-11$ & & & \\
\hline Er 149 & 148.9422 & $4.000 E+00$ & second & $4.00 \mathrm{E}+00$ & $1.8936 \mathrm{E}+10$ & $5.2808 \mathrm{E}-11$ & & & \\
\hline Er $149 m$ & 148.9422 & $8.900 \mathrm{E}+00$ & second & $8.90 E+00$ & $8.5108 E+09$ & $1.1750 E-10$ & & & \\
\hline Er 150 & 149.9378 & $1.850 \mathrm{E}+01$ & second & $1.85 \mathrm{E}+01$ & $4.0672 E+09$ & $2.4587 \mathrm{E}-10$ & & & \\
\hline Er 151 & 150.9317 & $2.350 E+01$ & second & $2.35 E+01$ & $3.1807 E+09$ & $3.1439 E-10$ & & & \\
\hline Er $151 \mathrm{~m}$ & 150.9317 & 5.800E-01 & second & $5.80 \mathrm{E}-01$ & $1.2887 E+11$ & $7.7595 \mathrm{E}-12$ & & & \\
\hline Er 152 & 151.9351 & $1.030 E+01$ & second & $1.03 \mathrm{E}+01$ & $7.2091 E+09$ & 1.3871E-10 & & & \\
\hline
\end{tabular}

WSRC-TR-99-00344

October, 1999

page 46 
Radionuclide Specific Data

\begin{tabular}{|c|c|c|c|c|c|c|c|c|c|}
\hline Isotope & $\begin{array}{l}\text { Atomic } \\
\text { Mass }\end{array}$ & Halflife & $\begin{array}{l}\text { Time } \\
\text { Units }\end{array}$ & $t_{1 / 2}, \sec$ & Curies/gram & Grams/ Curie & $\begin{array}{c}\text { Watts/Ci } \\
\text { (Ref.3) }\end{array}$ & $\begin{array}{c}\text { Individual }^{4} \\
\text { Fission } \\
\text { Product Yield } \\
(\text { Ref.4) }\end{array}$ & $\begin{array}{l}\text { Cumulative }^{1} \\
\text { Fission Product } \\
\text { Yield (Ref.4) }\end{array}$ \\
\hline Er 153 & 152.9351 & $3.710 E+01$ & second & $3.71 E+01$ & $1.9884 E+09$ & $5.0293 \mathrm{E}-10$ & & & \\
\hline Er 154 & 153.9328 & $3.730 \mathrm{E}+00$ & minute & $2.24 E+02$ & $3.2748 E+08$ & $3.0536 \mathrm{E}-09$ & & & \\
\hline Er 155 & 154.9332 & $5.300 E+00$ & minute & $3.18 E+02$ & $2.2898 \mathrm{E}+08$ & $4.3671 \mathrm{E}-09$ & & & \\
\hline \begin{tabular}{|l} 
Er 156 \\
\end{tabular} & 155.9310 & $1.950 \mathrm{E}+01$ & minute & $1.17 E+03$ & $6.1838 \mathrm{E}+07$ & $1.6171 \mathrm{E}-08$ & & & \\
\hline Er 157 & 156.9319 & $1.865 E+01$ & minute & $1.12 E+03$ & $6.4244 E+07$ & $1.5566 \mathrm{E}-08$ & & & \\
\hline Er $157 \mathrm{~m}$ & 156.9319 & $7.600 E-02$ & second & $7.60 \mathrm{E}-02$ & $9.4591 E+11$ & $1.0572 \mathrm{E}-12$ & & & \\
\hline Er 158 & 157.9299 & $2.290 E+00$ & hour & $8.24 \mathrm{E}+03$ & $8.6651 E+06$ & $1.1541 \mathrm{E}-07$ & & & \\
\hline Er 159 & 158.9307 & $3.600 \mathrm{E}+01$ & minute & $2.16 \mathrm{E}+03$ & $3.2864 \mathrm{E}+07$ & $3.0429 E-08$ & & & \\
\hline $\operatorname{Er} 160$ & 159.9291 & $2.858 E+01$ & hour & $1.03 E+05$ & $6.8562 E+05$ & $1.4585 \mathrm{E}-06$ & & & \\
\hline Er 161 & 160.9300 & $3.210 E+00$ & hour & $1.16 \mathrm{E}+04$ & $6.0664 E+06$ & $1.6484 \mathrm{E}-07$ & & & \\
\hline Er 163 & 162.9300 & $7.500 \mathrm{E}+01$ & minute & $4.50 \mathrm{E}+03$ & $1.5387 \mathrm{E}+07$ & $6.4989 \mathrm{E}-08$ & & $1.468 \mathrm{E}-21$ & $1.468 \mathrm{E}-21$ \\
\hline Er 165 & 164.9307 & $1.036 \mathrm{E}+01$ & hour & $3.73 E+04$ & $1.8341 E+06$ & $5.4524 \mathrm{E}-07$ & & $4.736 \mathrm{E}-18$ & $4.736 \mathrm{E}-18$ \\
\hline Er $167 m$ & 166.9320 & $2.269 E+00$ & second & $2.27 \mathrm{E}+00$ & $2.9785 E+10$ & $3.3574 E-11$ & & & \\
\hline Er 169 & 168.9346 & $9.400 \mathrm{E}+00$ & day & $8.12 E+05$ & $8.2227 E+04$ & $1.2161 \mathrm{E}-05$ & & $2.723 E-13$ & $2.246 \mathrm{E}-09$ \\
\hline Er 171 & 170.9380 & $7.516 \mathrm{E}+00$ & hour & $2.71 E+04$ & $2.4392 E+06$ & $4.0997 \mathrm{E}-07$ & & $2.193 E-12$ & $1.911 \mathrm{E}-10$ \\
\hline Er 172 & 171.9394 & $4.930 E+01$ & hour & $1.77 \mathrm{E}+05$ & $3.6970 E+05$ & $2.7049 \mathrm{E}-06$ & & $7.088 \mathrm{E}-12$ & $9.978 E-11$ \\
\hline Er 173 & 172.9424 & $1.400 \mathrm{E}+00$ & minute & $8.40 \mathrm{E}+01$ & $7.7660 \mathrm{E}+08$ & $1.2877 \mathrm{E}-09$ & & & \\
\hline Er 174 & 173.9443 & $3.300 \mathrm{E}+00$ & minute & $1.98 \mathrm{E}+02$ & $3.2757 E+08$ & $3.0528 \mathrm{E}-09$ & & & \\
\hline $\operatorname{Tm} 147$ & 146.9611 & $5.600 E-01$ & second & $5.60 \mathrm{E}-01$ & $1.3708 E+11$ & $7.2948 E-12$ & & & \\
\hline$T$ Tm 148 & 147.9576 & $7.000 \mathrm{E}-01$ & second & $7.00 \mathrm{E}-01$ & $1.0893 E+11$ & $9.1803 \mathrm{E}-12$ & & & \\
\hline $\operatorname{Tm} 149$ & 148.9527 & $9.000 \mathrm{E}-01$ & second & $9.00 \mathrm{E}-01$ & $8.4156 E+10$ & $1.1883 \mathrm{E}-11$ & & & \\
\hline $\operatorname{Tm} 150$ & 149.9497 & $2.200 E+00$ & second & $2.20 E+00$ & $3.4199 \mathrm{E}+10$ & $2.9241 \mathrm{E}-11$ & & & \\
\hline $\operatorname{Tm} 151$ & 150.9454 & $5.200 E+00$ & second & $5.20 \mathrm{E}+00$ & $1.4373 E+10$ & $6.9574 \mathrm{E}-11$ & & & \\
\hline$T \mathrm{~m} 151 \mathrm{~m}$ & 150.9454 & $4.130 E+00$ & second & $4.13 \mathrm{E}+00$ & $1.8097 \mathrm{E}+10$ & $5.5258 \mathrm{E}-11$ & & & \\
\hline $\operatorname{Tm} 152$ & 151.9443 & $5.200 \mathrm{E}+00$ & second & $5.20 \mathrm{E}+00$ & $1.4279 E+10$ & $7.0034 \mathrm{E}-11$ & & & \\
\hline $\operatorname{Tm} 152 \mathrm{~m}$ & 151.9443 & $8.000 E+00$ & second & $8.00 \mathrm{E}+00$ & $9.2812 \mathrm{E}+09$ & $1.0775 \mathrm{E}-10$ & & & \\
\hline$T \mathrm{Tm} 153$ & 152.9420 & $1.480 E+00$ & second & $1.48 \mathrm{E}+00$ & $4.9841 \mathrm{E}+10$ & $2.0064 \mathrm{E}-11$ & & & \\
\hline$T \mathrm{~m} 153 \mathrm{~m}$ & 152.9420 & $2.500 \mathrm{E}+00$ & second & $2.50 \mathrm{E}+00$ & $2.9506 \mathrm{E}+10$ & $3.3891 \mathrm{E}-11$ & & & 1 \\
\hline $\operatorname{Tm} 154$ & 153.9414 & $8.100 \mathrm{E}+00$ & second & $8.10 \mathrm{E}+00$ & $9.0477 E+09$ & 1.1053E-10 & & & \\
\hline Tm 154m & 153.9414 & $3.300 E+00$ & second & $3.30 E+00$ & $2.2208 E+10$ & $4.5029 \mathrm{E}-11$ & & & \\
\hline$T \mathrm{~m} 155$ & 154.9392 & $2.160 E+01$ & second & $2.16 \mathrm{E}+01$ & $3.3710 E+09$ & $2.9665 \mathrm{E}-10$ & & & \\
\hline $\operatorname{Tm} 155 \mathrm{~m}$ & 154.9392 & $4.500 E+01$ & second & $4.50 \mathrm{E}+01$ & $1.6181 E+09$ & $6.1801 E-10$ & & & \\
\hline $\operatorname{Tm} 156$ & 155.9390 & $8.380 E+01$ & second & $8.38 \mathrm{E}+01$ & $8.6333 E+08$ & $1.1583 \mathrm{E}-09$ & & & \\
\hline$T \mathrm{~m} 156 \mathrm{~m}$ & 155.9390 & $1.900 E+01$ & second & $1.90 \mathrm{E}+01$ & $3.8077 \mathrm{E}+09$ & $2.6262 \mathrm{E}-10$ & & & \\
\hline$T \mathrm{~m} 157$ & 156.9368 & $3.630 \mathrm{E}+00$ & minute & $2.18 \mathrm{E}+02$ & $3.3006 E+08$ & $3.0297 \mathrm{E}-09$ & & & \\
\hline
\end{tabular}

WSRC-TR-99-00344

October, 1999

page 47 


\begin{tabular}{|c|c|c|c|c|c|c|c|c|c|}
\hline Isotope & $\begin{array}{c}\text { Atomic } \\
\text { Mass }\end{array}$ & Halflife & $\begin{array}{l}\text { Time } \\
\text { Units }\end{array}$ & $t_{1 / 2}, \sec$ & Curies/gram & Grams/ Curie & $\begin{array}{c}\text { Watts/Ci } \\
\text { (Ref.3) }\end{array}$ & $\begin{array}{l}\text { Individual }^{1} \\
\text { Fission } \\
\text { Product Yield } \\
\text { (Ref.4) }\end{array}$ & $\begin{array}{c}\text { Cumulative' } \\
\text { Fission Product } \\
\text { Yield (Ref.4) }\end{array}$ \\
\hline $\mathrm{Tm} 158$ & 157.9370 & $3.980 E+00$ & minute & $2.39 E+02$ & $2.9913 E+08$ & $3.3430 E-09$ & & & \\
\hline $\mathrm{Tm} 159$ & 158.9348 & $9.130 E+00$ & minute & $5.48 E+02$ & $1.2958 E+08$ & $7.7173 \mathrm{E}-09$ & & & \\
\hline $\operatorname{Tm} 160$ & 159.9351 & $9.400 E+00$ & minute & $5.64 E+02$ & $1.2507 E+08$ & $7.9955 E-09$ & & & \\
\hline $\mathrm{Tm} 160 \mathrm{~m}$ & 159.9351 & $7.450 E+01$ & second & $7.45 \mathrm{E}+01$ & $9.4684 E+08$ & $1.0561 \mathrm{E}-09$ & & & \\
\hline $\mathrm{Tm} 161$ & 160.9334 & $3.020 E+01$ & minute & $1.81 E+03$ & $3.8688 E+07$ & $2.5848 E-08$ & & & \\
\hline $\operatorname{Tm} 162$ & 161.9340 & $2.170 E+01$ & minute & $1.30 \mathrm{E}+03$ & $5.3509 E+07$ & $1.8688 E-08$ & & & \\
\hline $\mathrm{Tm} 162$ & 161.9340 & $1.280 E+00$ & hour & $4.61 \mathrm{E}+03$ & $1.5119 E+07$ & $6.6141 \mathrm{E}-08$ & & & \\
\hline $\mathrm{Tm} 162 \mathrm{~m}$ & 161.9340 & $2.430 E+01$ & second & $2.43 E+01$ & $2.8670 E+09$ & $3.4879 E-10$ & & & \\
\hline $\operatorname{Tm} 163$ & 162.9326 & $1.810 E+00$ & hour & $6.52 E+03$ & $1.0626 \mathrm{E}+07$ & $9.4105 E-08$ & & & \\
\hline $\operatorname{Tr} 164$ & 163.9335 & $2.000 E+00$ & minute & $1.20 E+02$ & $5.7349 E+08$ & $1.7437 E-09$ & & & \\
\hline $\mathrm{Tm} 164 \mathrm{~m}$ & 163.9335 & $5.100 E+00$ & minute & $3.06 \mathrm{E}+02$ & $2.2490 E+08$ & $4.4464 \mathrm{E}-09$ & & & \\
\hline $\operatorname{Tm} 165$ & 164.9324 & $3.006 E+01$ & hour & $1.08 \mathrm{E}+05$ & $6.3209 E+05$ & 1.5821E-06 & & & \\
\hline $\operatorname{Tm} 166$ & 165.9336 & $7.700 E+00$ & hour & $2.77 \mathrm{E}+04$ & $2.4527 E+06$ & $4.0771 E-07$ & & & \\
\hline $\operatorname{Tm} 167$ & 166.9328 & $9.250 E+00$ & day & $7.99 \mathrm{E}+05$ & $8.4563 E+04$ & $1.1826 E-05$ & & & \\
\hline $\operatorname{Tm} 168$ & 167.9342 & $9.310 E+01$ & day & $8.04 E+06$ & $8.3517 E+03$ & $1.1974 E-04$ & & & \\
\hline $\operatorname{Tm} 170$ & 169.9358 & $1.286 E+02$ & day & $1.11 \mathrm{E}+07$ & $5.9750 E+03$ & $1.6736 \mathrm{E}-04$ & & $2.182 E-16$ & $2.182 E-16$ \\
\hline $\operatorname{Trm} 171$ & 170.9364 & $1.920 \mathrm{E}+00$ & year & $6.06 \mathrm{E}+07$ & $1.0892 E+03$ & $9.1807 E-04$ & & $2.0 .13 E-15$ & $1.911 \mathrm{E}-10$ \\
\hline $\operatorname{Tm} 172$ & 171.9384 & $6.360 \mathrm{E}+01$ & hour & $2.29 E+05$ & $2.8658 E+05$ & $3.4894 \mathrm{E}-06$ & & $1.872 \mathrm{E}-14$ & $9.980 \mathrm{E}-11$ \\
\hline $\operatorname{Tm} 173$ & 172.9396 & $8.240 E+00$ & hour & $2.97 \mathrm{E}+04$ & $2.1991 E+06$ & $4.5472 E-07$ & & & \\
\hline $\operatorname{Tm} 174$ & 173.9422 & $5.400 \mathrm{E}+00$ & minute & $3.24 E+02$ & $2.0018 \mathrm{E}+08$ & 4.9954E-09 & & & \\
\hline $\operatorname{Tm} 175$ & 174.9438 & $1.520 E+01$ & minute & $9.12 \mathrm{E}+02$ & $7.0710 E+07$ & $1.4142 E-08$ & & & \\
\hline $\operatorname{Tm} 176$ & 175.9470 & $1.900 \mathrm{E}+00$ & minute & $1.14 \mathrm{E}+02$ & $5.6246 E+08$ & $1.7779 E-09$ & & & \\
\hline $\operatorname{Tm} 177$ & 176.9490 & $8.500 E+01$ & second & $8.50 \mathrm{E}+01$ & $7.5008 E+08$ & $1.3332 \mathrm{E}-09$ & & & \\
\hline Yb 151 & 150.9552 & $1.600 \mathrm{E}+00$ & second & $1.60 \mathrm{E}+00$ & $4.6710 E+10$ & $2.1409 \mathrm{E}-11$ & & & \\
\hline Yb 151m & 150.9552 & $1.600 E+00$ & second & $1.60 E+00$ & $4.6710 E+10$ & $2.1409 \mathrm{E}-11$ & & & \\
\hline $\mathrm{Yb} 152$ & 151.9502 & $3.100 E+00$ & second & $3.10 E+00$ & $2.3950 E+10$ & $4.1753 \mathrm{E}-11$ & & & \\
\hline Yb 153 & 152.9420 & $4.200 E+00$ & second & $4.20 \mathrm{E}+00$ & $1.7563 E+10$ & $5.6938 \mathrm{E}-11$ & & & \\
\hline Yb 154 & 153.9462 & 4.040E-01 & second & 4.04E-01 & $1.8140 \mathrm{E}+11$ & $5.5128 \mathrm{E}-12$ & & & \\
\hline Yb 155 & 154.9458 & $1.750 \mathrm{E}+00$ & second & $1.75 \mathrm{E}+00$ & $4.1606 E+10$ & $2.4035 \mathrm{E}-11$ & & & \\
\hline Yb 156 & 155.9428 & $2.610 E+01$ & second & $2.61 \mathrm{E}+01$ & $2.7719 E+09$ & $3.6077 E-10$ & & & \\
\hline Yb 157 & 156.9427 & $3.860 E+01$ & second & $3.86 E+01$ & $1.8623 E+09$ & $5.3697 E-10$ & & & \\
\hline Yb 158 & 157.9399 & $1.490 \mathrm{E}+00$ & minute & $8.94 \mathrm{E}+01$ & $7.9900 E+08$ & $1.2516 \mathrm{E}-09$ & & & \\
\hline Yb 159 & 158.9402 & $1.580 \mathrm{E}+00$ & minute & $9.48 E+01$ & $7.4875 \mathrm{E}+08$ & $1.3356 \mathrm{E}-09$ & & & \\
\hline Yb 160 & 159.9376 & $4.800 \mathrm{E}+00$ & minute & $2.88 \mathrm{E}+02$ & $2.4493 E+08$ & $4.0829 E-09$ & & & \\
\hline Yb 161 & 160.9379 & $4.200 E+00$ & minute & $2.52 E+02$ & $2.7817 E+08$ & $3.5949 E-09$ & & & \\
\hline
\end{tabular}

WSRC-TR-99-00344

October, 1999

page 48 


\begin{tabular}{|c|c|c|c|c|c|c|c|c|c|}
\hline Isotope & $\begin{array}{l}\text { Atomic } \\
\text { Mass }\end{array}$ & Halflife & $\begin{array}{l}\text { Time } \\
\text { Units }\end{array}$ & $t_{1 / 2}, \sec$ & Curies/gram & Grams/ Curie & $\begin{array}{c}\text { Watts/Ci } \\
\text { (Ref.3) }\end{array}$ & $\begin{array}{c}\text { Individual }^{1} \\
\text { Fission } \\
\text { Product Yield } \\
\text { (Ref.4) } \\
\end{array}$ & $\begin{array}{c}\text { Cumulative } \\
\text { Fission Product } \\
\text { Yield (Ref.4) }\end{array}$ \\
\hline Yb 162 & 161.9358 & $1.887 E+01$ & minute & $1.13 E+03$ & $6.1533 \mathrm{E}+07$ & $1.6251 \mathrm{E}-08$ & & & \\
\hline Yb 163 & 162.9363 & $1.105 E+01$ & minute & $6.63 E+02$ & $1.0443 E+08$ & $9.5754 \mathrm{E}-09$ & & & \\
\hline Yb 164 & 163.9345 & $7.580 E+01$ & minute & $4.55 E+03$ & $1.5132 E+07$ & $6.6087 \mathrm{E}-08$ & & & \\
\hline Yb 165 & 164.9354 & $9.900 E+00$ & minute & $5.94 \mathrm{E}+02$ & $1.1515 \mathrm{E}+08$ & $8.6841 \mathrm{E}-09$ & & & \\
\hline Yb 166 & 165.9339 & $5.670 \mathrm{E}+01$ & hour & $2.04 \mathrm{E}+05$ & $3.3309 E+05$ & $3.0022 \mathrm{E}-06$ & & & \\
\hline Yb 167 & 166.9349 & $1.750 \mathrm{E}+01$ & minute & $1.05 E+03$ & $6.4364 \mathrm{E}+07$ & $1.5537 \mathrm{E}-08$ & & & \\
\hline Yb 169 & 168.9352 & $3.203 E+01$ & day & $2.77 E+06$ & $2.4135 E+04$ & 4.1434E-05 & 0.002512 & $2.927 \mathrm{E}-22$ & $2.927 \mathrm{E}-22$ \\
\hline Yb 169m & 168.9352 & $4.600 E+01$ & second & $4.60 E+01$ & $1.4518 \mathrm{E}+09$ & $6.8881 E-10$ & & & \\
\hline Yb 175 & 174.9413 & $4.185 E+00$ & day & $3.62 E+05$ & $1.7835 E+05$ & $5.6069 \mathrm{E}-06$ & & & \\
\hline Yb 176m & 175.9426 & $1.140 E+01$ & second & $1.14 E+01$ & $5.6247 E+09$ & $1.7779 \mathrm{E}-10$ & & & \\
\hline Yb 177 & 176.9453 & $1.911 \mathrm{E}+00$ & hour & $6.88 \mathrm{E}+03$ & $9.2678 \mathrm{E}+06$ & $1.0790 \mathrm{E}-07$ & & & \\
\hline Yb 177m & 176.9453 & $6.410 \mathrm{E}+00$ & second & $6.41 \mathrm{E}+00$ & $9.9467 E+09$ & $1.0054 \mathrm{E}-10$ & & & \\
\hline Yb 178 & 177.9466 & $7.400 E+01$ & minute & $4.44 E+03$ & $1.4279 \mathrm{E}+07$ & $7.0032 \mathrm{E}-08$ & & & \\
\hline Yb 179 & 178.9502 & $8.000 E+00$ & minute & $4.80 E+02$ & $1.3134 \mathrm{E}+08$ & $7.6137 \mathrm{E}-09$ & & & \\
\hline Yb 180 & 179.9523 & $2.400 \mathrm{E}+00$ & minute & $1.44 E+02$ & $4.3537 E+08$ & 2.2969E-09 & & & \\
\hline Lu 151 & 150.9671 & $8.500 \mathrm{E}-02$ & second & $8.50 \mathrm{E}-02$ & $8.7917 \mathrm{E}+11$ & $1.1374 E-12$ & & & \\
\hline Lu 152 & 151.9636 & $7.000 \mathrm{E}-01$ & second & $7.00 \mathrm{E}-01$ & $1.0606 \mathrm{E}+11$ & $9.4289 \mathrm{E}-12$ & & & \\
\hline Lu 154 & 153.9571 & $1.120 E+00$ & second & $1.12 \mathrm{E}+00$ & $6.5427 E+10$ & $1.5284 \mathrm{E}-11$ & & & \\
\hline Lu 155 & 154.9542 & $1.400 \mathrm{E}-01$ & second & $1.40 \mathrm{E}-01$ & $5.2005 \mathrm{E}+11$ & $1.9229 \mathrm{E}-12$ & & & \\
\hline Lu $155 \mathrm{~m}$ & 154.9542 & $6.800 \mathrm{E}-02$ & second & $6.80 \mathrm{E}-02$ & $1.0707 E+12$ & $9.3398 \mathrm{E}-13$ & & & \\
\hline Lu $155 \mathrm{~m} 2$ & 154.9542 & $2.600 \mathrm{E}-03$ & second & $2.60 \mathrm{E}-03$ & $2.8003 E+13$ & $3.5711 \mathrm{E}-14$ & & & \\
\hline Lu 156 & 155.9529 & $7.300 \mathrm{E}-01$ & second & 7.30E-01 & $9.9097 E+10$ & $1.0091 \mathrm{E}-11$ & & & \\
\hline Lu 157 & 156.9501 & $6.800 E+00$ & second & $6.80 E+00$ & $1.0571 \mathrm{E}+10$ & $9.4601 \mathrm{E}-11$ & & & \\
\hline Lu 157m & 156.9501 & $4.790 E+00$ & second & $4.79 E+00$ & $1.5006 \mathrm{E}+10$ & $6.6638 \mathrm{E}-11$ & & & \\
\hline Lu 158 & 157.9492 & $1.040 \mathrm{E}+01$ & second & $1.04 E+01$ & $6.8679 \mathrm{E}+09$ & $1.4560 \mathrm{E}-10$ & & & \\
\hline Lu 159 & 158.9466 & $1.210 E+01$ & second & $1.21 E+01$ & $5.8660 \mathrm{E}+09$ & $1.7047 \mathrm{E}-10$ & & & \\
\hline Lu 160 & 159.9460 & $3.610 \mathrm{E}+01$ & second & $3.61 \mathrm{E}+01$ & $1.9539 \mathrm{E}+09$ & $5.1180 \mathrm{E}-10$ & & & \\
\hline Lu $160 \mathrm{~m}$ & 159.9460 & $4.000 E+01$ & second & $4.00 E+01$ & $1.7634 \mathrm{E}+09$ & $5.6710 \mathrm{E}-10$ & & & \\
\hline Lu 161 & 160.9435 & $7.200 E+01$ & second & $7.20 \mathrm{E}+01$ & $9.7358 E+08$ & $1.0271 \mathrm{E}-09$ & & & \\
\hline Lu $161 \mathrm{~m}$ & 160.9435 & $7.300 \mathrm{E}-03$ & second & $7.30 \mathrm{E}-03$ & $9.6024 \mathrm{E}+12$ & $1.0414 \mathrm{E}-13$ & & & \\
\hline Lu 162 & 161.9432 & $1.370 E+00$ & minute & $8.22 E+01$ & $8.4750 \mathrm{E}+08$ & 1.1799E-09 & & & \\
\hline Lu $162 \mathrm{~m}$ & 161.9432 & $1.500 \mathrm{E}+00$ & minute & $9.00 E+01$ & $7.7405 E+08$ & $1.2919 \mathrm{E}-09$ & & & \\
\hline Lu $162 \mathrm{~m} 2$ & 161.9432 & $1.900 \mathrm{E}+00$ & minute & $1.14 \mathrm{E}+02$ & $6.1110 \mathrm{E}+08$ & $1.6364 \mathrm{E}-09$ & & & \\
\hline Lu 163 & 162.9412 & $2.380 E+02$ & second & $2.38 \mathrm{E}+02$ & $2.9092 E+08$ & $3.4374 \mathrm{E}-09$ & & & \\
\hline Lu 164 & 163.9412 & $3.140 \mathrm{E}+00$ & minute & $1.88 \mathrm{E}+02$ & $3.6526 \mathrm{E}+08$ & $2.7377 \mathrm{E}-09$ & & & \\
\hline
\end{tabular}




\begin{tabular}{|c|c|c|c|c|c|c|c|c|c|}
\hline Isotope & $\begin{array}{l}\text { Atomic } \\
\text { Mass }\end{array}$ & Halflife & $\begin{array}{l}\text { Time } \\
\text { Units }\end{array}$ & $t_{1 / 2}, \sec$ & Curies/ gram & Grams/ Curie & $\begin{array}{c}\text { Watts/Ci } \\
\text { (Ref.3) }\end{array}$ & $\begin{array}{c}\text { Individual }^{1} \\
\text { Fission } \\
\text { Product Yield } \\
\text { (Ref.4) }\end{array}$ & $\begin{array}{l}\text { Cumulative }^{1} \\
\text { Fission Product } \\
\text { Yleld (Ref.4) }\end{array}$ \\
\hline Lu 165 & 164.9396 & $1.074 E+01$ & minute & $6.44 E+02$ & $1.0614 E+08$ & $9.4212 \mathrm{E}-09$ & & & \\
\hline Lu $165 \mathrm{~m}$ & 164.9396 & $1.200 \mathrm{E}+01$ & minute & $7.20 E+02$ & $9.4999 \mathrm{E}+07$ & $1.0526 \mathrm{E}-08$ & & & \\
\hline Lu 166 & 165.9398 & $2.650 E+00$ & minute & $1.59 E+02$ & $4.2759 \mathrm{E}+08$ & 2.3387E-09 & & & \\
\hline Lu $166 \mathrm{~m}$ & 165.9398 & $1.410 E+00$ & minute & $8.46 E+01$ & $8.0363 E+08$ & $1.2444 \mathrm{E}-09$ & & & \\
\hline Lu $166 \mathrm{~m} 2$ & 165.9398 & $2.120 E+00$ & minute & $1.27 E+02$ & $5.3449 \mathrm{E}+08$ & $1.8709 \mathrm{E}-09$ & & & \\
\hline Lu 167 & 166.9383 & $5.150 \mathrm{E}+01$ & minute & $3.09 E+03$ & $2.1871 E+07$ & $4.5723 E-08$ & & & \\
\hline Lu 168 & 167.9387 & $5.500 \mathrm{E}+00$ & minute & $3.30 \mathrm{E}+02$ & $2.0357 E+08$ & 4.9123E-09 & & & \\
\hline Lu $168 \mathrm{~m}$ & 167.9387 & $6.700 E+00$ & minute & $4.02 E+02$ & $1.6711 \mathrm{E}+08$ & $5.9841 \mathrm{E}-09$ & & & \\
\hline Lu 169 & 168.9376 & $3.406 \mathrm{E}+01$ & hour & $1.23 E+05$ & $5.4463 E+05$ & $1.8361 \mathrm{E}-06$ & & & \\
\hline Lu 169m & 168.9376 & $1.600 E+02$ & second & $1.60 E+02$ & $4.1738 E+08$ & $2.3959 \mathrm{E}-09$ & & & \\
\hline Lu 170 & 169.9385 & $2.000 E+00$ & day & $1.73 E+05$ & $3.8419 E+05$ & $2.6029 \mathrm{E}-06$ & & & \\
\hline Lu 170m & 169.9385 & $6.700 \mathrm{E}-01$ & second & $6.70 \mathrm{E}-01$ & $9.9085 \mathrm{E}+10$ & $1.0092 E-11$ & & & \\
\hline Lu 171 & 170.9379 & $8.240 \mathrm{E}+00$ & day & $7.12 E+05$ & $9.2704 E+04$ & $1.0787 \mathrm{E}-05$ & & & \\
\hline Lu $171 \mathrm{~m}$ & 170.9379 & $7.900 E+01$ & second & $7.90 E+01$ & $8.3543 E+08$ & $1.1970 \mathrm{E}-09$ & & & \\
\hline Lu 172 & 171.9391 & $6.700 \mathrm{E}+00$ & day & $5.79 \mathrm{E}+05$ & $1.1335 E+05$ & $8.8224 E-06$ & & & \\
\hline Lu 172m & 171.9391 & $3.700 \mathrm{E}+00$ & minute & $2.22 E+02$ & $2.9556 E+08$ & $3.3834 E-09$ & & & \\
\hline Lu 173 & 172.9389 & $1.370 E+00$ & year & $4.32 \mathrm{E}+07$ & $1.5089 E+03$ & $6.6275 \mathrm{E}-04$ & & & \\
\hline Lu 174 & 173.9403 & $3.310 E+00$ & year & $1.04 E+08$ & $6.2092 E+02$ & $1.6105 E-03$ & & & \\
\hline Lu 174m & 173.9403 & $1.420 E+02$ & day & $1.23 E+07$ & $5.2866 E+03$ & $1.8916 E-04$ & & & \\
\hline Lu 176 & 175.9427 & $3.780 E+10$ & year & $1.19 E+18$ & $5.3752 \mathrm{E}-08$ & $1.8604 E+07$ & & & \\
\hline Lu 176m & 175.9427 & $3.635 \mathrm{E}+00$ & hour & $1.31 E+04$ & $4.9000 \mathrm{E}+06$ & $2.0408 \mathrm{E}-07$ & & & \\
\hline Lu 177 & 176.9438 & $6.734 \mathrm{E}+00$ & day & $5.82 E+05$ & $1.0959 \mathrm{E}+05$ & $9.1253 \mathrm{E}-06$ & & & \\
\hline Lu $177 \mathrm{~m}$ & 176.9438 & $1.604 E+02$ & day & $1.39 E+07$ & $4.6007 E+03$ & $2.1736 \mathrm{E}-04$ & & & \\
\hline Lu 178 & 177.9460 & $2.840 E+01$ & minute & $1.70 E+03$ & $3.7206 E+07$ & $2.6877 E-08$ & & & \\
\hline Lu $178 \mathrm{~m}$ & 177.9460 & $2.310 E+01$ & minute & $1.39 E+03$ & $4.5743 E+07$ & $2.1861 \mathrm{E}-08$ & & & \\
\hline Lu 179 & 178.9473 & $4.590 \mathrm{E}+00$ & hour & $1.65 \mathrm{E}+04$ & $3.8154 E+06$ & $2.6210 \mathrm{E}-07$ & & & \\
\hline Lu $179 \mathrm{~m}$ & 178.9473 & $3.100 \mathrm{E}-03$ & second & $3.10 \mathrm{E}-03$ & $2.0337 E+13$ & $4.9171 \mathrm{E}-14$ & & & \\
\hline Lu 180 & 179.9499 & $5.700 E+00$ & minute & $3.42 E+02$ & $1.8332 E+08$ & $5.4551 \mathrm{E}-09$ & & & \\
\hline \begin{tabular}{|ll} 
Lu 181 \\
\end{tabular} & 180.9520 & $3.500 E+00$ & minute & $2.10 \mathrm{E}+02$ & $2.9689 E+08$ & $3.3683 \mathrm{E}-09$ & & & \\
\hline Lu 182 & 181.9506 & $2.000 E+00$ & minute & $1.20 E+02$ & $5.1670 \mathrm{E}+08$ & 1.9353E-09 & & & \\
\hline LL 183 & 182.9576 & $5.800 \mathrm{E}+01$ & second & $5.80 \mathrm{E}+01$ & $1.0632 \mathrm{E}+09$ & $9.4059 \mathrm{E}-10$ & & & \\
\hline \begin{tabular}{|ll}
$L U$ & 184
\end{tabular} & 183.9612 & $2.000 E+01$ & second & $2.00 E+01$ & $3.0663 E+09$ & $3.2612 \mathrm{E}-10$ & & & \\
\hline Hf 154 & 153.9643 & $2.000 \mathrm{E}+00$ & second & $2.00 \mathrm{E}+00$ & $3.6638 \mathrm{E}+10$ & $2.7294 \mathrm{E}-11$ & & & \\
\hline Hf 155 & 154.9542 & $8.900 \mathrm{E}-01$ & second & $8.90 \mathrm{E}-01$ & $8.1806 E+10$ & $1.2224 \mathrm{E}-11$ & & & \\
\hline Hf 156 & 155.9529 & $2.500 \mathrm{E}-02$ & second & $2.50 \mathrm{E}-02$ & $2.8936 E+12$ & $3.4559 \mathrm{E}-13$ & & & \\
\hline
\end{tabular}

WSRC-TR-99-00344

October, 1999

page 50 
Radionuclide Specific Data

\begin{tabular}{|c|c|c|c|c|c|c|c|c|c|}
\hline Isotope & $\begin{array}{l}\text { Atomic } \\
\text { Mass }\end{array}$ & Halflife & $\begin{array}{l}\text { Time } \\
\text { Units }\end{array}$ & $t_{122}, \sec$ & Curies/ gram & Grams/ Curie & $\begin{array}{c}\text { Watts/Ci } \\
\text { (Ref.3) }\end{array}$ & $\begin{array}{c}\text { Individual' } \\
\text { Fission } \\
\text { Product Yield } \\
\text { (Ref.4) }\end{array}$ & $\begin{array}{c}\text { Cumulative } \\
\text { Fission Product } \\
\text { Yield (Ref.4) }\end{array}$ \\
\hline Hf 157 & 156.9581 & $1.100 \mathrm{E}-01$ & second & 1.10E-01 & $6.5343 E+11$ & $1.5304 \mathrm{E}-12$ & & & \\
\hline Hf 158 & 157.9546 & $2.860 E+00$ & second & $2.86 E+00$ & $2.4973 E+10$ & $4.0043 \mathrm{E}-11$ & & & \\
\hline Hf 159 & 158.9540 & $5.600 \mathrm{E}+00$ & second & $5.60 E+00$ & $1.2674 \mathrm{E}+10$ & $7.8901 \mathrm{E}-11$ & & & \\
\hline Hf 160 & 159.9507 & $1.300 \mathrm{E}+01$ & second & $1.30 E+01$ & $5.4256 E+09$ & $1.8431 \mathrm{E}-10$ & & & \\
\hline Hf 161 & 160.9503 & $1.680 \mathrm{E}+01$ & second & $1.68 \mathrm{E}+01$ & $4.1723 E+09$ & $2.3968 \mathrm{E}-10$ & & & \\
\hline Hf 162 & 161.9472 & $3.760 E+01$ & second & $3.76 \mathrm{E}+01$ & $1.8527 \mathrm{E}+09$ & $5.3974 \mathrm{E}-10$ & & & \\
\hline Hf 163 & 162.9471 & $4.000 E+01$ & second & $4.00 E+01$ & $1.7309 \mathrm{E}+09$ & $5.7774 \mathrm{E}-10$ & & & \\
\hline Hf 164 & 163.9444 & $1.110 \mathrm{E}+02$ & second & $1.11 E+02$ & $6.1995 E+08$ & $1.6130 \mathrm{E}-09$ & & & \\
\hline $\mathrm{Hf} 165$ & 164.9445 & $7.600 E+01$ & second & $7.60 \mathrm{E}+01$ & $8.9996 \mathrm{E}+08$ & $1.1112 \mathrm{E}-09$ & & & \\
\hline Hf 166 & 165.9423 & $6.770 E+00$ & minute & $4.06 E+02$ & $1.6737 E+08$ & $5.9748 E-09$ & & & \\
\hline Hf 167 & 166.9426 & $2.050 E+00$ & minute & $1.23 E+02$ & $5.4942 E+08$ & $1.8201 \mathrm{E}-09$ & & & \\
\hline Hf 168 & 167.9406 & $2.595 \mathrm{E}+01$ & minute & $1.56 E+03$ & $4.3145 E+07$ & $2.3178 \mathrm{E}-08$ & & & \\
\hline Hf 169 & 168.9412 & $3.240 E+00$ & minute & $1.94 \mathrm{E}+02$ & $3.4351 E+08$ & $2.9111 \mathrm{E}-09$ & & & \\
\hline Hf 170 & 169.9397 & $1.601 E+01$ & hour & $5.76 E+04$ & $1.1518 E+06$ & $8.6818 \mathrm{E}-07$ & & & \\
\hline Hf 171 & 170.9405 & $1.210 E+01$ & hour & $4.36 \mathrm{E}+04$ & $1.5151 E+06$ & $6.6002 \mathrm{E}-07$ & & & \\
\hline Hf 172 & 171.9395 & $1.870 E+00$ & year & $5.90 \mathrm{E}+07$ & $1.1118 E+03$ & $8.9941 \mathrm{E}-04$ & & & \\
\hline Hf 173 & 172.9407 & $2.360 E+01$ & hour & $8.50 E+04$ & $7.6783 E+05$ & $1.3024 E-06$ & & & \\
\hline Hf 174 & 173.9400 & $2.000 E+15$ & year & $6.31 \mathrm{E}+22$ & $1.0276 \mathrm{E}-12$ & $9.7313 E+11$ & & & \\
\hline Hf 175 & 174.9415 & $7.000 E+01$ & day & $6.05 E+06$ & $1.0663 E+04$ & $9.3784 E-05$ & 0.002422 & & \\
\hline $\mathrm{Hf} 177 \mathrm{~m}$ & 176.9432 & $1.080 E+00$ & second & $1.08 E+00$ & $5.9036 \mathrm{E}+10$ & $1.6939 \mathrm{E}-11$ & & & \\
\hline Hf $177 \mathrm{~m} 2$ & 176.9432 & $5.140 E+01$ & minute & $3.08 E+03$ & $2.0674 \mathrm{E}+07$ & $4.8370 \mathrm{E}-08$ & & & \\
\hline Hf $178 \mathrm{~m}$ & 177.9437 & $4.000 E+00$ & second & $4.00 \mathrm{E}+00$ & $1.5850 \mathrm{E}+10$ & $6.3091 \mathrm{E}-11$ & & & \\
\hline Hf $178 \mathrm{~m} 2$ & 177.9437 & $3.100 E+01$ & year & $9.78 \mathrm{E}+08$ & $6.4806 \mathrm{E}+01$ & $1.5431 \mathrm{E}-02$ & & & \\
\hline $\mathrm{Hf} 179 \mathrm{~m}$ & 178.9458 & $1.867 E+01$ & second & $1.87 E+01$ & $3.3768 E+09$ & $2.9613 \mathrm{E}-10$ & & & \\
\hline $\mathrm{Hf} 179 \mathrm{~m} 2$ & 178.9458 & $2.505 E+01$ & day & $2.16 \mathrm{E}+06$ & $2.9130 \mathrm{E}+04$ & $3.4329 \mathrm{E}-05$ & & & \\
\hline $\mathrm{Hf} 180 \mathrm{~m}$ & 179.9465 & $5.500 \mathrm{E}+00$ & hour & $1.98 E+04$ & $3.1664 E+06$ & $3.1581 \mathrm{E}-07$ & & & \\
\hline Hf 181 & 180.9491 & $4.239 E+01$ & day & $3.66 \mathrm{E}+06$ & $1.7023 E+04$ & $5.8743 E-05$ & 0.004470 & & \\
\hline Hf 182 & 181.9506 & $9.000 \mathrm{E}+06$ & year & $2.84 E+14$ & $2.1831 \mathrm{E}-04$ & $4.5807 \mathrm{E}+03$ & & & 1 \\
\hline Hf $182 m$ & 181.9506 & $6.150 E+01$ & minute & $3.69 E+03$ & $1.6803 E+07$ & $5.9512 E-08$ & & & \\
\hline Hf 183 & 182.9514 & $1.067 E+00$ & hour & $3.84 E+03$ & $1.6054 E+07$ & $6.2291 \mathrm{E}-08$ & & & \\
\hline Hf 184 & 183.9554 & $4.120 \mathrm{E}+00$ & hour & $1.48 E+04$ & $4.1349 \mathrm{E}+06$ & $2.4184 \mathrm{E}-07$ & & & \\
\hline Hf 185 & 184.9556 & $3.500 E+00$ & minute & $2.10 E+02$ & $2.9046 E+08$ & $3.4428 \mathrm{E}-09$ & & & \\
\hline Ta 156 & 155.9717 & $1.100 \mathrm{E}-01$ & second & $1.10 \mathrm{E}-01$ & $6.5756 \mathrm{E}+11$ & $1.5208 \mathrm{E}-12$ & & & \\
\hline Ta 157 & 156.9681 & $5.300 \mathrm{E}-03$ & second & $5.30 \mathrm{E}-03$ & $1.3561 E+13$ & $7.3741 E-14$ & & & \\
\hline Ta 158 & 157.9664 & $3.680 \mathrm{E}-02$ & second & $3.68 \mathrm{E}-02$ & $1.9407 E+12$ & $5.1527 \mathrm{E}-13$ & & & \\
\hline
\end{tabular}

WSRC-TR-99-00344

October, 1999

page 51 


\begin{tabular}{|c|c|c|c|c|c|c|c|c|c|}
\hline Isotope & $\begin{array}{l}\text { Atomic } \\
\text { Mass }\end{array}$ & Halflife & $\begin{array}{l}\text { Time } \\
\text { Units }\end{array}$ & $t_{1 / 2}, \sec$ & Curies/gram & Grams/ Curie & $\begin{array}{c}\text { Watts/Ci } \\
\text { (Ref.3) }\end{array}$ & $\begin{array}{c}\text { Individual }^{1} \\
\text { Flssion } \\
\text { Product Yield } \\
\text { (Ref.4) } \\
\end{array}$ & $\begin{array}{c}\text { Cumulative } \\
\text { Fission Product } \\
\text { Yield (Ref.4) }\end{array}$ \\
\hline Ta 159 & 158.9629 & $5.700 \mathrm{E}-01$ & second & $5.70 E-01$ & $1.2451 \mathrm{E}+11$ & $8.0315 E-12$ & & & \\
\hline Ta 160 & 159.9614 & $1.500 \mathrm{E}+00$ & second & $1.50 E+00$ & $4.7019 \mathrm{E}+10$ & $2.1268 \mathrm{E}-11$ & & & \\
\hline Ta 161 & 160.9584 & $2.700 \mathrm{E}+00$ & second & $2.70 E+00$ & $2.5960 E+10$ & $3.8521 E-11$ & & & \\
\hline Ta 162 & 161.9571 & $3.520 \mathrm{E}+00$ & second & $3.52 \mathrm{E}+00$ & $1.9789 E+10$ & $5.0532 E-11$ & & & \\
\hline Ta 163 & 162.9543 & $1.060 \mathrm{E}+01$ & second & $1.06 E+01$ & $6.5314 E+09$ & $1.5311 \mathrm{E}-10$ & & & \\
\hline Ta 164 & 163.9536 & $1.420 \mathrm{E}+01$ & second & $1.42 E+01$ & $4.8458 \mathrm{E}+09$ & $2.0636 E-10$ & & & \\
\hline Ta 165 & 164.9508 & $3.100 E+01$ & second & $3.10 \mathrm{E}+01$ & $2.2063 E+09$ & $4.5325 \mathrm{E}-10$ & & & \\
\hline Ta 166 & 165.9505 & $3.440 \mathrm{E}+01$ & second & $3.44 E+01$ & $1.9762 E+09$ & $5.0601 E-10$ & & & \\
\hline Ta 167 & 166.9480 & $1.330 \mathrm{E}+00$ & minute & $7.98 \mathrm{E}+01$ & $8.4682 E+08$ & 1.1809E-09 & & & \\
\hline Ta 168 & 167.9478 & $2.000 E+00$ & minute & $1.20 E+02$ & $5.5978 \mathrm{E}+08$ & $1.7864 E-09$ & & & \\
\hline Ta 169 & 168.9459 & $4.900 E+00$ & minute & $2.94 E+02$ & $2.2713 E+08$ & $4.4027 E-09$ & & & \\
\hline Ta 170 & 169.9461 & $6.760 \mathrm{E}+00$ & minute & $4.06 \mathrm{E}+02$ & $1.6367 E+08$ & $6.1099 \mathrm{E}-09$ & & & \\
\hline Ta 171 & 170.9445 & $2.330 \mathrm{E}+01$ & minute & $1.40 \mathrm{E}+03$ & $4.7208 \mathrm{E}+07$ & $2.1183 E-08$ & & & \\
\hline Ta 172 & 171.9447 & $3.680 E+01$ & minute & $2.21 E+03$ & $2.9716 \mathrm{E}+07$ & $3.3652 E-08$ & & & \\
\hline Ta 173 & 172.9435 & $3.140 \mathrm{E}+00$ & hour & $1.13 E+04$ & $5.7709 \mathrm{E}+06$ & $1.7328 \mathrm{E}-07$ & & & \\
\hline Ta 174 & 173.9442 & $1.050 \mathrm{E}+00$ & hour & $3.78 E+03$ & $1.7158 \mathrm{E}+07$ & $5.8281 E-08$ & & & \\
\hline Ta 175 & 174.9437 & $1.050 \mathrm{E}+01$ & hour & $3.78 E+04$ & $1.7060 \mathrm{E}+06$ & $5.8616 \mathrm{E}-07$ & & & \\
\hline Ta 176 & 175.9447 & $8.090 \mathrm{E}+00$ & hour & $2.91 \mathrm{E}+04$ & $2.2017 \mathrm{E}+06$ & $4.5420 \mathrm{E}-07$ & & & \\
\hline Ta 177 & 176.9445 & $5.656 \mathrm{E}+01$ & hour & $2.04 E+05$ & $3.1313 E+05$ & $3.1935 \mathrm{E}-06$ & & & \\
\hline Ta 178 & 177.9458 & $9.310 \mathrm{E}+00$ & minute & $5.59 \mathrm{E}+02$ & $1.1350 \mathrm{E}+08$ & 8.8107E-09 & & & \\
\hline Ta $178 \mathrm{~m}$ & 177.9458 & $2.360 \mathrm{E}+00$ & hour & $8.50 E+03$ & $7.4623 E+06$ & $1.3401 \mathrm{E}-07$ & & & \\
\hline Ta 178m2 & 177.9458 & $6.000 \mathrm{E}-02$ & second & $6.00 \mathrm{E}-02$ & $1.0567 \mathrm{E}+12$ & $9.4637 \mathrm{E}-13$ & & & \\
\hline Ta 179 & 178.9459 & $1.820 \mathrm{E}+00$ & year: & $5.74 E+07$ & $1.0977 E+03$ & $9.1103 E-04$ & & & \\
\hline Ta 180 & 179.9475 & $8.152 \mathrm{E}+00$ & hour & $2.93 E+04$ & $2.1363 \mathrm{E}+06$ & $4.6810 \mathrm{E}-07$ & & & \\
\hline Ta $180 \mathrm{~m}$ & 179.9475 & $1.200 \mathrm{E}+15$ & year & $3.79 \mathrm{E}+22$ & $1.6555 \mathrm{E}-12$ & $6.0404 E+11$ & & & \\
\hline Ta 182 & 181.9502 & $1.144 E+02$ & day & $9.89 \mathrm{E}+06$ & $6.2715 E+03$ & $1.5945 \mathrm{E}-04$ & 0.008940 & & \\
\hline Ta $182 \mathrm{~m}$ & 181.9502 & $2.830 \mathrm{E}-01$ & second & $2.83 \mathrm{E}-01$ & $2.1910 \mathrm{E}+11$ & $4.5642 E-12$ & & & \\
\hline Ta $182 \mathrm{~m} 2$ & 181.9502 & $1.584 E+01$ & minute & $9.50 \mathrm{E}+02$ & $6.5241 \mathrm{E}+07$ & $1.5328 \mathrm{E}-08$ & & & \\
\hline Ta 183 & 182.9514 & $5.100 \mathrm{E}+00$ & day & $4.41 E+05$ & $1.3994 E+05$ & $7.1457 \mathrm{E}-06$ & & & \\
\hline Ta 184 & 183.9540 & $8.700 \mathrm{E}+00$ & hour & $3.13 \mathrm{E}+04$ & $1.9581 E+06$ & $5.1069 \mathrm{E}-07$ & & & \\
\hline Ta 185 & 184.9556 & $4.940 \mathrm{E}+01$ & minute & $2.96 E+03$ & $2.0579 \mathrm{E}+07$ & $4.8592 E-08$ & & & \\
\hline Ta 186 & 185.9586 & $1.050 \mathrm{E}+01$ & minute & $6.30 \mathrm{E}+02$ & $9.6299 \mathrm{E}+07$ & $1.0384 \mathrm{E}-08$ & & & \\
\hline W 158 & 157.9739 & $9.000 \mathrm{E}-04$ & second & $9.00 \mathrm{E}-04$ & $7.9350 \mathrm{E}+13$ & $1.2602 \mathrm{E}-14$ & & & \\
\hline W 159 & 158.9723 & $7.300 \mathrm{E}-03$ & second & $7.30 \mathrm{E}-03$ & $9.7215 \mathrm{E}+12$ & $1.0287 \mathrm{E}-13$ & & & \\
\hline W 160 & 159.9684 & $8.100 \mathrm{E}-02$ & second & $8.10 \mathrm{E}-02$ & $8.7068 \mathrm{E}+11$ & $1.1485 E-12$ & & & \\
\hline
\end{tabular}

WSRC-TR-99-00344

October, 1999

page 52 
Radionuclide Specific Data

\begin{tabular}{|c|c|c|c|c|c|c|c|c|c|}
\hline Isotope & $\begin{array}{l}\text { Atomic } \\
\text { Mass }\end{array}$ & Halflife & $\begin{array}{l}\text { Time } \\
\text { Units }\end{array}$ & $t_{1 / 2}, \sec$ & Curies/ gram & Grams/Curie & $\begin{array}{c}\text { Watts/Ci } \\
\text { (Ref.3) }\end{array}$ & $\begin{array}{c}\text { Individual }^{1} \\
\text { Fission } \\
\text { Product Yield } \\
\text { (Ref.4) }\end{array}$ & $\begin{array}{l}\text { Cumulative } \\
\text { Fission Product } \\
\text { Yield (Ref.4) }\end{array}$ \\
\hline W 161 & 160.9671 & $4.100 \mathrm{E}-01$ & second & $4.10 \mathrm{E}-01$ & $1.7094 \mathrm{E}+11$ & $5.8498 \mathrm{E}-12$ & & & \\
\hline W 162 & 161.9633 & $1.390 E+00$ & second & $1.39 E+00$ & $5.0112 E+10$ & $1.9955 \mathrm{E}-11$ & & & \\
\hline$W 163$ & 162.9625 & $2.750 E+00$ & second & $2.75 E+00$ & $2.5174 E+10$ & 3.9723E-11 & & & \\
\hline W 164 & 163.9590 & $6.000 E+00$ & second & $6.00 \mathrm{E}+00$ & $1.1468 \mathrm{E}+10$ & $8.7199 \mathrm{E}-11$ & & & \\
\hline W 165 & 164.9583 & $5.100 E+00$ & second & $5.10 E+00$ & $1.3410 \mathrm{E}+10$ & $7.4571 \mathrm{E}-11$ & & & \\
\hline W 166 & 165.9550 & $1.880 \mathrm{E}+01$ & second & $1.88 \mathrm{E}+01$ & $3.6160 E+09$ & $2.7655 \mathrm{E}-10$ & & & \\
\hline W 167 & 166.9547 & $1.990 E+01$ & second & $1.99 E+01$ & $3.3957 \mathrm{E}+09$ & $2.9449 \mathrm{E}-10$ & & & \\
\hline W 168 & 167.9519 & $5.100 E+01$ & second & $5.10 E+01$ & $1.3171 E+09$ & $7.5924 \mathrm{E}-10$ & & & \\
\hline$W 169$ & 168.9518 & $7.600 \mathrm{E}+01$ & second & $7.60 E+01$ & $8.7862 E+08$ & $1.1382 \mathrm{E}-09$ & & & \\
\hline W 170 & 169.9493 & $4.000 E+00$ & minute & $2.40 E+02$ & $2.7660 \mathrm{E}+08$ & $3.6154 \mathrm{E}-09$ & & & \\
\hline W 171 & 170.9495 & $2.380 E+00$ & minute & $1.43 E+02$ & $4.6215 \mathrm{E}+08$ & $2.1638 \mathrm{E}-09$ & & & \\
\hline W 172 & 171.9474 & $6.600 E+00$ & minute & $3.96 E+02$ & $1.6569 \mathrm{E}+08$ & $6.0355 \mathrm{E}-09$ & & & \\
\hline W 173 & 172.9478 & $7.600 E+00$ & minute & $4.56 \mathrm{E}+02$ & $1.4305 \mathrm{E}+08$ & $6.9904 \mathrm{E}-09$ & & & \\
\hline W174 & 173.9462 & $3.100 E+01$ & minute & $1.86 \mathrm{E}+03$ & $3.4870 \mathrm{E}+07$ & $2.8678 \mathrm{E}-08$ & & & \\
\hline W 175 & 174.9468 & $3.520 E+01$ & minute & $2.11 E+03$ & $3.0534 \mathrm{E}+07$ & $3.2751 \mathrm{E}-08$ & & & \\
\hline W 176 & 175.9456 & $2.500 E+00$ & hour & $9.00 \mathrm{E}+03$ & $7.1245 E+06$ & $1.4036 \mathrm{E}-07$ & & & \\
\hline W 177 & 176.9466 & $1.350 E+02$ & minute & $8.10 E+03$ & $7.8713 E+06$ & $1.2704 \mathrm{E}-07$ & & & \\
\hline W 178 & 177.9458 & $2.160 E+01$ & day & $1.87 \mathrm{E}+06$ & $3.3972 E+04$ & $2.9436 \mathrm{E}-05$ & & & \\
\hline W179 & 178.9471 & $3.705 E+01$ & minute & $2.22 E+03$ & $2.8360 \mathrm{E}+07$ & $3.5260 \mathrm{E}-08$ & & & \\
\hline W 179m & 178.9471 & $6.400 E+00$ & minute & $3.84 E+02$ & $1.6418 \mathrm{E}+08$ & $6.0909 \mathrm{E}-09$ & & & \\
\hline W $180 \mathrm{~m}$ & 179.9467 & $5.470 \mathrm{E}-03$ & second & $5.47 E-03$ & $1.1462 E+13$ & $8.7248 \mathrm{E}-14$ & & & \\
\hline W 181 & 180.9482 & $1.212 E+02$ & day & $1.05 E+07$ & $5.9540 \mathrm{E}+03$ & 1.6796E-04 & & & \\
\hline W183 & 182.9502 & $1.100 E+17$ & year & $3.47 E+24$ & $1.7764 E-14$ & $5.6294 E+13$ & & & \\
\hline W $183 \mathrm{~m}$ & 182.9502 & $5.200 \mathrm{E}+00$ & second & $5.20 E+00$ & $1.1859 \mathrm{E}+10$ & $8.4326 \mathrm{E}-11$ & & & \\
\hline W 184 & 183.9509 & $3.000 E+17$ & year & $9.47 E+24$ & $6.4779 \mathrm{E}-15$ & $1.5437 E+14$ & & & \\
\hline W185 & 184.9534 & $7.510 E+01$ & day & $6.49 E+06$ & $9.4007 \mathrm{E}+03$ & $1.0637 E-04$ & & & \\
\hline W $185 \mathrm{~m}$ & 184.9534 & $1.670 E+00$ & minute & $1.00 \mathrm{E}+02$ & $6.0876 \mathrm{E}+08$ & $1.6427 \mathrm{E}-09$ & & & \\
\hline W 187 & 186.9572 & $2.372 E+01$ & hour & $8.54 \mathrm{E}+04$ & $7.0667 \mathrm{E}+05$ & 1.4151E-06 & & & \\
\hline W 188 & 187.9585 & $6.940 E+01$ & day & $6.00 \mathrm{E}+06$ & $1.0010 E+04$ & $9.9898 \mathrm{E}-05$ & & & i \\
\hline W189 & 188.9619 & $1.150 E+01$ & minute & $6.90 E+02$ & $8.6527 \mathrm{E}+07$ & 1.1557E-08 & & & \\
\hline$W 190$ & 189.9632 & $3.000 E+01$ & minute & $1.80 \mathrm{E}+03$ & $3.2994 E+07$ & $3.0309 \mathrm{E}-08$ & & & \\
\hline $\operatorname{Re} 160$ & 159.9815 & $7.900 \mathrm{E}-04$ & second & $7.90 E-04$ & $8.9265 E+13$ & $1.1203 \mathrm{E}-14$ & & & \\
\hline $\operatorname{Re} 161$ & 160.9777 & $1.000 \mathrm{E}-02$ & second & $1.00 E-02$ & $7.0083 E+12$ & $1.4269 \mathrm{E}-13$ & & & \\
\hline $\operatorname{Re} 162$ & 161.9757 & $1.000 \mathrm{E}-01$ & second & $1.00 \mathrm{E}-01$ & $6.9651 \mathrm{E}+11$ & $1.4357 \mathrm{E}-12$ & & & \\
\hline $\operatorname{Re} 163$ & 162.9720 & $2.600 \mathrm{E}-01$ & second & $2.60 \mathrm{E}-01$ & $2.6625 \mathrm{E}+11$ & $3.7559 \mathrm{E}-12$ & & & \\
\hline
\end{tabular}

WSRC-TR-99-00344

October, 1999

page 53 


\begin{tabular}{|c|c|c|c|c|c|c|c|c|c|c|c|c|c|c|c|c|c|c|c|c|c|c|c|c|c|c|c|c|c|c|c|c|c|}
\hline 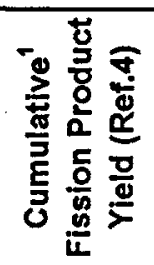 & & & & & & & & & & & & & & & & & & & & & & & & & & & & & & & & & \\
\hline 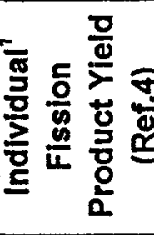 & & & & & & & & & & & & & & & & & & & & & & & & & & & & & & & & & \\
\hline 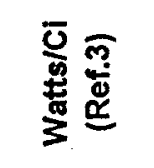 & & & & & & & & & & & & & & & & & & & & & & & & & & & & & & & & & \\
\hline 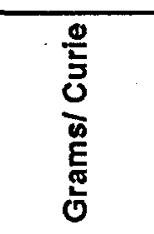 & 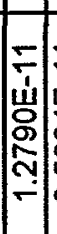 & 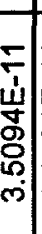 & 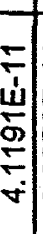 & 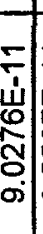 & 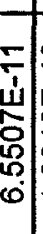 & 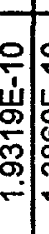 & 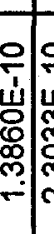 & 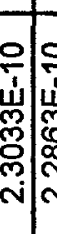 & 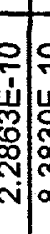 & 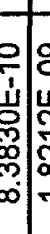 & & 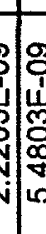 & 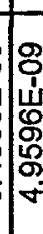 & 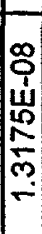 & | & 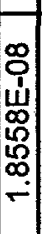 & 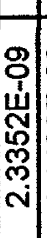 & 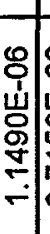 & 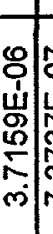 & 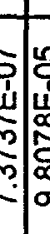 & 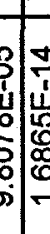 & 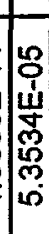 & 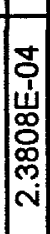 & $\begin{array}{l}0 \\
0 \\
u \\
w \\
0 \\
0 \\
0 \\
0 \\
0\end{array}$ & 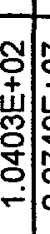 & 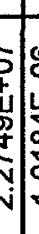 & $\because$ & & & $\approx$ & & 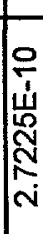 & \\
\hline $\begin{array}{l}E \\
E \\
\frac{E}{50} \\
\bar{g} \\
\frac{0}{5} \\
0 \\
0\end{array}$ & 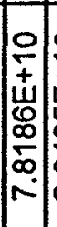 & 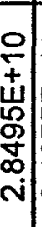 & 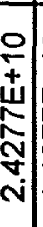 & \begin{tabular}{l|}
0 \\
$\vdots$ \\
$\omega$ \\
5 \\
0 \\
\\
\end{tabular} & 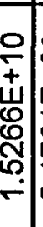 & 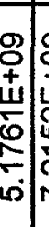 & 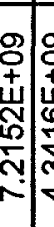 & 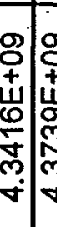 & 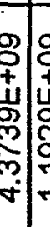 & 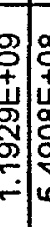 & 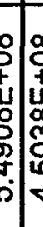 & 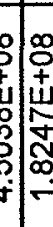 & 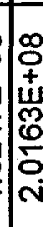 & 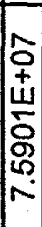 & 跄 & 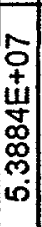 & 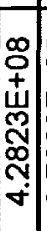 & 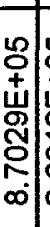 & 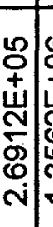 & 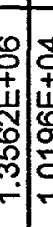 & 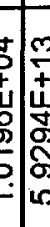 & 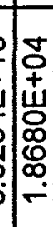 & 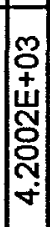 & 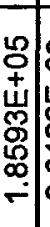 & 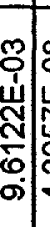 & 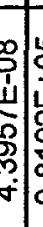 & 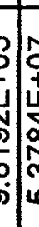 & 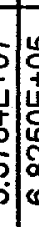 & 210 & 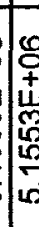 & 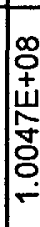 & 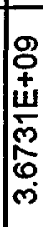 & 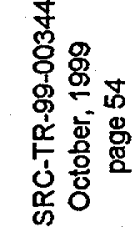 \\
\hline 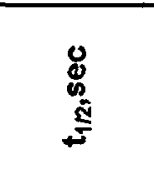 & 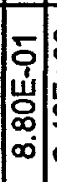 & 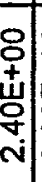 & 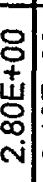 & 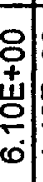 & $\begin{array}{l}8 \\
0 \\
\dot{4} \\
\\
\vdots \\
\dot{\forall}\end{array}$ & 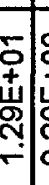 & 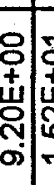 & 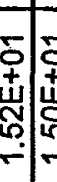 & 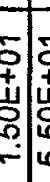 & 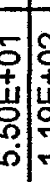 & 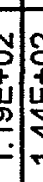 & 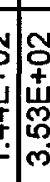 & 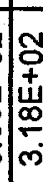 & 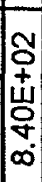 & 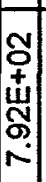 & 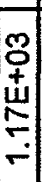 & . & 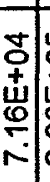 & 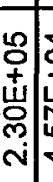 & 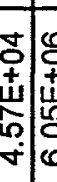 & 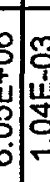 & 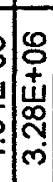 & 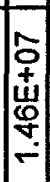 & 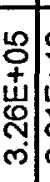 & 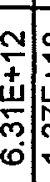 & 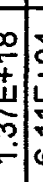 & & & 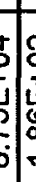 & y & 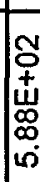 & 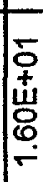 & \\
\hline 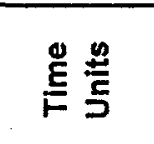 & $\mid$ & 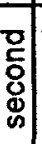 & 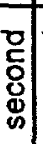 & 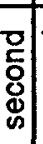 & 밈 & $\begin{array}{l}0 \\
\vdots \\
\vdots \\
\vdots \\
0\end{array}$ & 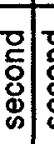 & \begin{tabular}{l|l} 
\\
$\vdots$ \\
$\vdots$
\end{tabular} & \begin{tabular}{l|l}
0 \\
\\
\end{tabular} & 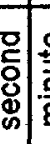 & & 兽 & 竞 & 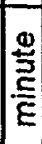 & 量 & . & 를 & 능 & 흥 & ؛े & 웡 & 3 & $>$ & 홍 & $\stackrel{\frac{1}{8}}{2}$ & 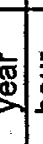 & & & & ठิ & & $\begin{array}{l}0 \\
\\
\\
0 \\
0 \\
0\end{array}$ & \\
\hline 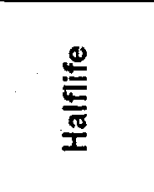 & 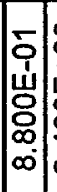 & 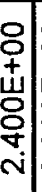 & $\begin{array}{c}8 \\
+ \\
+ \\
\\
8 \\
\infty \\
-1\end{array}$ & $\begin{array}{l}8 \\
8 \\
+ \\
\\
8 \\
\\
0 \\
\end{array}$ & $\begin{array}{c}8 \\
0 \\
+ \\
\\
0 \\
\vdots \\
\dot{v}\end{array}$ & 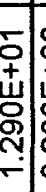 & 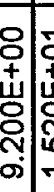 & 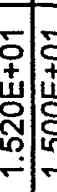 & 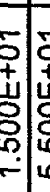 & 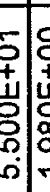 & & 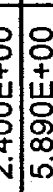 & 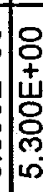 & 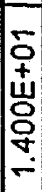 & 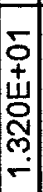 & 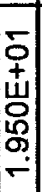 & 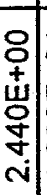 & 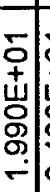 & $\begin{array}{l}- \\
+ \\
\vdots \\
\vdots \\
\vdots \\
\vdots \\
\vdots\end{array}$ & 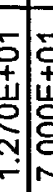 & 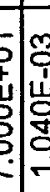 & 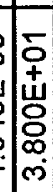 & 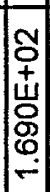 & 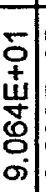 & 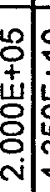 & 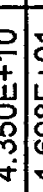 & - & $\dot{ }=$ & & 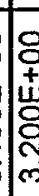 & ' & 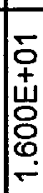 & \\
\hline 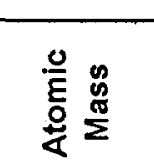 & 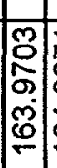 & $\begin{array}{l}-5 \\
6 \\
9 \\
0 \\
0\end{array}$ & \begin{tabular}{l|}
0 \\
0 \\
0 \\
0 \\
0 \\
0
\end{tabular} & \begin{tabular}{l}
0 \\
0 \\
8 \\
8 \\
8 \\
\hdashline
\end{tabular} & \begin{tabular}{l|}
0 \\
0 \\
8 \\
$\vdots$ \\
0 \\
-
\end{tabular} & 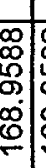 & 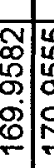 & 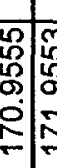 & 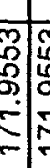 & 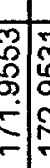 & & $\begin{array}{l}5 \\
\vdots \\
\vdots \\
\vdots \\
\end{array}$ & 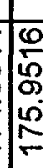 & 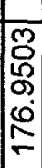 & 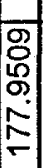 & 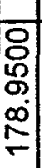 & 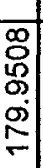 & $\begin{array}{l}-5 \\
8 \\
9 \\
8 \\
0\end{array}$ & $\begin{array}{l}\frac{v}{5} \\
\frac{5}{\circ} \\
\frac{\infty}{\sigma}\end{array}$ & 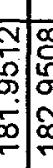 & 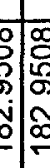 & 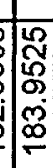 & 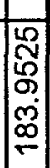 & $\begin{array}{l}0 \\
0 \\
\tilde{0} \\
\sigma \\
\infty \\
\infty \\
\sigma \\
\sigma\end{array}$ & $\begin{array}{l}\delta \\
0 \\
0 \\
0 \\
\infty \\
\infty \\
\infty\end{array}$ & $\mathbb{R}^{\circ}$ & 5 & & & $\frac{\infty}{\infty}$ & $\begin{array}{l}\overline{8} \\
8 \\
8 \\
8\end{array}$ & $\mid$\begin{tabular}{l}
8 \\
8 \\
8 \\
5 \\
$\sigma$ \\
\hdashline
\end{tabular} & \\
\hline 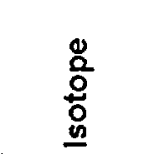 & $\left|\begin{array}{l}\mid \\
0 \\
0 \\
0 \\
\alpha\end{array}\right|$ & $\begin{array}{l}0 \\
0 \\
\alpha \\
\alpha\end{array}$ & \begin{tabular}{l}
8 \\
$\vdots$ \\
\hdashline \\
\hdashline
\end{tabular} & $\begin{array}{l}\hat{o} \\
\hat{\alpha} \\
\alpha\end{array}$ & $\begin{array}{l}\infty \\
0 \\
0 \\
\alpha\end{array}$ & $\begin{array}{l}\stackrel{\varrho}{0} \\
\Phi \\
\alpha\end{array}$ & $\begin{array}{l}\frac{\pi}{\alpha} \\
\alpha \\
\alpha\end{array}$ & $\begin{array}{l}\underset{\alpha}{\alpha} \\
\stackrel{\alpha}{\alpha}\end{array}$ & \begin{tabular}{l}
$=$ \\
\hdashline \\
$\alpha$ \\
$\alpha$
\end{tabular} & 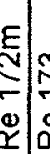 & & $=\frac{n}{\infty}$ & $\stackrel{0}{\sigma}$ & $\begin{array}{l}\hat{N} \\
\approx \\
0 \\
\alpha \\
\alpha\end{array}$ & 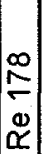 & 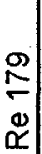 & $\begin{array}{l}0 \\
0 \\
\infty \\
\alpha\end{array}$ & $\underset{\infty}{-}$ & $\underset{\infty}{\infty}$ & 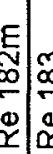 & $\frac{?}{0}$ & 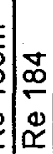 & $\begin{array}{l}\frac{5}{5} \\
\infty \\
-\end{array}$ & $\left.\begin{array}{c}\mathscr{0} \\
\infty \\
\hdashline \\
0 \\
\alpha\end{array}\right]$ & \begin{tabular}{c}
$E$ \\
0 \\
$\infty$ \\
\hdashline
\end{tabular} & $\infty$ & & & & ક̊ & & & \\
\hline
\end{tabular}


Radionuclide Specific Data

\begin{tabular}{|c|c|c|c|c|c|c|c|c|c|}
\hline Isotope & $\begin{array}{l}\text { Atomic } \\
\text { Mass }\end{array}$ & Halflife & $\begin{array}{l}\text { Time } \\
\text { Units }\end{array}$ & $t_{1 / 2}, \sec$ & Curies/gram & Grams/ Curie & $\begin{array}{c}\text { Watts/Ci } \\
\text { (Ref.3) }\end{array}$ & $\begin{array}{c}\text { Individual }^{4} \\
\text { Fission } \\
\text { Product Yield } \\
\text { (Ref.4) } \\
\end{array}$ & $\begin{array}{c}\text { Cumulative } \\
\text { Fission Product } \\
\text { Yield (Ref.4) }\end{array}$ \\
\hline Os 162 & 161.9757 & $1.900 \mathrm{E}-03$ & second & $1.90 \mathrm{E}-03$ & $3.6658 \mathrm{E}+13$ & $2.7279 \mathrm{E}-14$ & & & \\
\hline Os 164 & 163.9779 & $4.100 \mathrm{E}-02$ & second & $4.10 \mathrm{E}-02$ & $1.6781 E+12$ & $5.9593 \mathrm{E}-13$ & & & \\
\hline Os 165 & 164.9765 & $6.500 \mathrm{E}-02$ & second & $6.50 \mathrm{E}-02$ & $1.0521 \mathrm{E}+12$ & $9.5051 \mathrm{E}-13$ & & & \\
\hline Os 166 & 165.9725 & $1.810 \mathrm{E}-01$ & second & $1.81 \mathrm{E}-01$ & $3.7554 \mathrm{E}+11$ & $2.6628 \mathrm{E}-12$ & & & \\
\hline Os 167 & 166.9716 & $8.300 \mathrm{E}-01$ & second & $8.30 \mathrm{E}-01$ & $8.1406 E+10$ & $1.2284 \mathrm{E}-11$ & & & \\
\hline \begin{tabular}{|l|} 
Os 168 \\
\end{tabular} & 167.9678 & $2.200 \mathrm{E}+00$ & second & $2.20 E+00$ & $3.0530 \mathrm{E}+10$ & $3.2755 \mathrm{E}-11$ & & & \\
\hline Os 169 & 168.9671 & $3.400 E+00$ & second & $3.40 \mathrm{E}+00$ & $1.9638 \mathrm{E}+10$ & $5.0922 \mathrm{E}-11$ & & & \\
\hline Os 170 & 169.9636 & $7.300 \mathrm{E}+00$ & second & $7.30 E+00$ & $9.0928 \mathrm{E}+09$ & $1.0998 \mathrm{E}-10$ & & & \\
\hline Os 171 & 170.9630 & $8.000 E+00$ & second & $8.00 E+00$ & $8.2487 E+09$ & $1.2123 \mathrm{E}-10$ & & & \\
\hline Os 172 & 171.9601 & $1.900 E+01$ & second & $1.90 \mathrm{E}+01$ & $3.4530 \mathrm{E}+09$ & $2.8960 \mathrm{E}-10$ & & & \\
\hline Os 173 & 172.9598 & $1.600 \mathrm{E}+01$ & second & $1.60 \mathrm{E}+01$ & $4.0767 E+09$ & $2.4529 \mathrm{E}-10$ & & & \\
\hline Os 174 & 173.9571 & $4.400 E+01$ & second & $4.40 \mathrm{E}+01$ & $1.4739 E+09$ & $6.7845 \mathrm{E}-10$ & & & \\
\hline Os 175 & 174.9571 & $1.400 E+00$ & minute & $8.40 E+01$ & $7.6765 E+08$ & $1.3027 \mathrm{E}-09$ & & & \\
\hline Os 176 & 175.9550 & $3.600 \mathrm{E}+00$ & minute & $2.16 \mathrm{E}+02$ & $2.9684 E+08$ & $3.3688 \mathrm{E}-09$ & & & \\
\hline Os 177 & 176.9550 & $2.800 E+00$ & minute & $1.68 \mathrm{E}+02$ & $3.7949 E+08$ & $2.6351 \mathrm{E}-09$ & & & \\
\hline Os 178 & 177.9533 & $5.000 E+00$ & minute & $3.00 \mathrm{E}+02$ & $2.1132 \mathrm{E}+08$ & $4.7321 \mathrm{E}-09$ & & & \\
\hline Os 179 & 178.9540 & $6.500 \mathrm{E}+00$ & minute & $3.90 \mathrm{E}+02$ & $1.6165 E+08$ & $6.1863 \mathrm{E}-09$ & & & \\
\hline Os 180 & 179.9524 & $2.150 E+01$ & minute & $1.29 \mathrm{E}+03$ & $4.8599 \mathrm{E}+07$ & $2.0576 \mathrm{E}-08$ & & & \\
\hline 0 S 181 & 180.9533 & $1.050 E+02$ & minute & $6.30 \mathrm{E}+03$ & $9.8962 E+06$ & $1.0105 \mathrm{E}-07$ & & & \\
\hline Os $181 \mathrm{~m}$ & 180.9533 & $2.700 \mathrm{E}+00$ & minute & $1.62 E+02$ & $3.8485 E+08$ & $2.5984 \mathrm{E}-09$ & & & \\
\hline Os 182 & 181.9522 & $2.210 E+01$ & hour & $7.96 E+04$ & $7.7934 E+05$ & $1.2831 \mathrm{E}-06$ & & & \\
\hline Os 183 & 182.9531 & $1.300 \mathrm{E}+01$ & hour & $4.68 E+04$ & $1.3176 \mathrm{E}+06$ & 7.5894E-07 & & & \\
\hline Os $183 \mathrm{~m}$ & 182.9531 & $9.900 E+00$ & hour. & $3.56 E+04$ & $1.7302 E+06$ & $5.7796 \mathrm{E}-07$ & & & \\
\hline Os 184 & 183.9525 & $5.600 \mathrm{E}+13$ & year & $1.77 E+21$ & $3.4703 \mathrm{E}-11$ & $2.8816 \mathrm{E}+10$ & & & \\
\hline Os 185 & 184.9540 & $9.360 \mathrm{E}+01$ & day & $8.09 E+06$ & $7.5426 \mathrm{E}+03$ & $1.3258 \mathrm{E}-04$ & & & \\
\hline Os 186 & 185.9538 & $2.000 E+15$ & year & $6.31 E+22$ & $9.6123 \mathrm{E}-13$ & $1.0403 E+12$ & & & \\
\hline Os $189 \mathrm{~m}$ & 188.9581 & $5.800 \mathrm{E}+00$ & hour & $2.09 E+04$ & $2.8594 \mathrm{E}+06$ & $3.4972 \mathrm{E}-07$ & & & \\
\hline Os 190m & 189.9584 & $9.900 E+00$ & minute & $5.94 E+02$ & $9.9984 E+07$ & $1.0002 E-08$ & & & \\
\hline Os 191 & 190.9609 & $1.540 E+01$ & day & $1.33 \mathrm{E}+06$ & $4.4401 \mathrm{E}+04$ & $2.2522 \mathrm{E}-05$ & & & \\
\hline Os $191 \mathrm{~m}$ & 190.9609 & $1.310 \mathrm{E}+01$ & hour & $4.72 E+04$ & $1.2527 \mathrm{E}+06$ & $7.9826 \mathrm{E}-07$ & & & \\
\hline Os 192m & 191.9615 & $5.900 \mathrm{E}+00$ & second & $5.90 \mathrm{E}+00$ & $9.9612 \mathrm{E}+09$ & $1.0039 \mathrm{E}-10$ & & & \\
\hline Os 193 & 192.9641 & $3.050 E+01$ & hour & $1.10 \mathrm{E}+05$ & $5.3247 \mathrm{E}+05$ & $1.8780 \mathrm{E}-06$ & & & \\
\hline Os 194 & 193.9652 & $6.000 \mathrm{E}+00$ & year & $1.89 \mathrm{E}+08$ & $3.0717 \mathrm{E}+02$ & $3.2555 \mathrm{E}-03$ & & & \\
\hline Os 195 & 194.9681 & $6.500 \mathrm{E}+00$ & minute & $3.90 E+02$ & $1.4837 E+08$ & $6.7399 \mathrm{E}-09$ & & & \\
\hline Os 196 & 195.9696 & $3.490 \mathrm{E}+01$ & minute & $2.09 E+03$ & $2.7492 \mathrm{E}+07$ & $3.6374 \mathrm{E}-08$ & & & \\
\hline
\end{tabular}

WSRC-TR-99-00344

October, 1999

page 55 


\begin{tabular}{|c|c|c|c|c|c|c|c|c|c|}
\hline Isotope & $\begin{array}{l}\text { Atomic } \\
\text { Mass }\end{array}$ & Halflife & $\begin{array}{l}\text { Time } \\
\text { Units }\end{array}$ & $t_{1 / 2}, \sec$ & Curies/ gram & Grams/ Curie & $\begin{array}{c}\text { Watts/Ci } \\
\text { (Ref.3) }\end{array}$ & $\begin{array}{c}\text { Individual }^{1} \\
\text { Fission } \\
\text { Product Yield } \\
\text { (Ref.4) }\end{array}$ & $\begin{array}{c}\text { Cumulative } \\
\text { Fission Product } \\
\text { Yield (Ret.4) } \\
\end{array}$ \\
\hline II 166 & 165.9855 & $5.000 \mathrm{E}-03$ & second & $5.00 \mathrm{E}-03$ & $1.3594 \mathrm{E}+13$ & $7.3564 \mathrm{E}-14$ & & & \\
\hline Ir 167 & 166.9815 & $5.000 \mathrm{E}-03$ & second & $5.00 \mathrm{E}-03$ & $1.3513 E+13$ & $7.4005 \mathrm{E}-14$ & & & \\
\hline $\mid$ & 168.9764 & $4.000 \mathrm{E}-01$ & second & $4.00 \mathrm{E}-01$ & $1.6691 \mathrm{E}+11$ & $5.9911 E-12$ & & & \\
\hline If 170 & 169.9750 & $1.050 E+00$ & second & $1.05 \mathrm{E}+00$ & $6.3212 E+10$ & $1.5820 \mathrm{E}-11$ & & & \\
\hline Ir 171 & 170.9718 & $1.500 \mathrm{E}+00$ & second & $1.50 \mathrm{E}+00$ & $4.3991 \mathrm{E}+10$ & $2.2732 \mathrm{E}-11$ & & & \\
\hline Ir 172 & 171.9706 & $4.400 E+00$ & second & $4.40 \mathrm{E}+00$ & $1.4910 \mathrm{E}+10$ & $6.7070 \mathrm{E}-11$ & & & \\
\hline Ir $172 \mathrm{~m}$ & 171.9706 & $2.000 E+00$ & second & $2.00 \mathrm{E}+00$ & $3.2801 \mathrm{E}+10$ & $3.0487 E-11$ & & & \\
\hline II 173 & 172.9677 & $9.000 E+00$ & second & $9.00 \mathrm{E}+00$ & $7.2472 E+09$ & $1.3798 \mathrm{E}-10$ & & & \\
\hline Ir $173 m$ & 172.9677 & $2.200 E+00$ & second & $2.20 E+00$ & $2.9648 E+10$ & $3.3730 \mathrm{E}-11$ & & & \\
\hline \begin{tabular}{|l|}
$\operatorname{lr} 174$ \\
\end{tabular} & 173.9668 & $4.000 \mathrm{E}+00$ & second & $4.00 \mathrm{E}+00$ & $1.6213 \mathrm{E}+10$ & $6.1681 \mathrm{E}-11$ & & & \\
\hline II 175 & 174.9643 & $9.000 E+00$ & second & $9.00 E+00$ & $7.1645 \mathrm{E}+09$ & $1.3958 \mathrm{E}-10$ & & & \\
\hline If 176 & 175.9635 & $8.000 E+00$ & second & $8.00 \mathrm{E}+00$ & $8.0143 E+09$ & $1.2478 \mathrm{E}-10$ & & & \\
\hline Ir 177 & 176.9612 & $3.000 \mathrm{E}+01$ & second & $3.00 \mathrm{E}+01$ & $2.1251 \mathrm{E}+09$ & $4.7057 \mathrm{E}-10$ & & & \\
\hline If 178 & 177.9611 & $1.200 E+01$ & second & $1.20 E+01$ & $5.2829 \mathrm{E}+09$ & $1.8929 \mathrm{E}-10$ & & & \\
\hline Ir 179 & 178.9592 & $7.900 \mathrm{E}+01$ & second & $7.90 \mathrm{E}+01$ & $7.9799 \mathrm{E}+08$ & $1.2532 \mathrm{E}-09$ & & & \\
\hline II 180 & 179.9593 & $1.500 E+00$ & minute & $9.00 \mathrm{E}+01$ & $6.9656 \mathrm{E}+08$ & $1.4356 \mathrm{E}-09$ & & & \\
\hline If 181 & 180.9576 & $4.900 \mathrm{E}+00$ & minute & $2.94 \mathrm{E}+02$ & $2.1206 \mathrm{E}+08$ & 4.7157E-09 & & & \\
\hline $\operatorname{lr} 182$ & 181.9581 & $1.500 E+01$ & minute & $9.00 \mathrm{E}+02$ & $6.8891 E+07$ & $1.4516 \mathrm{E}-08$ & & & \\
\hline Ir 183 & 182.9568 & $5.800 \mathrm{E}+01$ & minute & $3.48 E+03$ & $1.7719 \mathrm{E}+07$ & $5.6435 \mathrm{E}-08$ & & & \\
\hline Ir 184 & 183.9574 & $3.090 \mathrm{E}+00$ & hour & $1.11 \mathrm{E}+04$ & $5.5131 \mathrm{E}+06$ & $1.8139 \mathrm{E}-07$ & & & \\
\hline $\mid 18185$ & 184.9566 & $1.440 E+01$ & hour & $5.18 E+04$ & $1.1766 \mathrm{E}+06$ & $8.4988 \mathrm{E}-07$ & & & 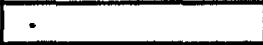 \\
\hline Ir 186 & 185.9580 & $1.664 \mathrm{E}+01$ & hour & $5.99 E+04$ & $1.0128 \mathrm{E}+06$ & $9.8740 \mathrm{E}-07$ & & & \\
\hline Ir $186 \mathrm{~m}$ & 185.9580 & $2.000 \mathrm{E}+00$ & hour & $7.20 \mathrm{E}+03$ & $8.4262 E+06$ & $1.1868 \mathrm{E}-07$ & & & \\
\hline Ir 187 & 186.9574 & $1.050 \mathrm{E}+01$ & hour & $3.78 E+04$ & $1.5964 E+06$ & $6.2641 \mathrm{E}-07$ & & & \\
\hline Ir $187 \mathrm{~m}$ & 186.9574 & $3.030 E-02$ & second & $3.03 E-02$ & $1.9916 \mathrm{E}+12$ & $5.0212 \mathrm{E}-13$ & & & \\
\hline Ir 188 & 187.9589 & $4.150 E+01$ & hour & $1.49 E+05$ & $4.0176 \mathrm{E}+05$ & $2.4891 \mathrm{E}-06$ & & & \\
\hline Ir 189 & 188.9587 & $1.320 E+01$ & day & $1.14 E+06$ & $5.2351 \mathrm{E}+04$ & $1.9102 \mathrm{E}-05$ & & & \\
\hline If $189 \mathrm{~m}$ & 188.9587 & $1.330 \mathrm{E}-02$ & second & $1.33 \mathrm{E}-02$ & $4.4891 \mathrm{E}+12$ & $2.2276 \mathrm{E}-13$ & & & \\
\hline Ir 190 & 189.9606 & $1.178 \mathrm{E}+01$ & day & $1.02 E+06$ & $5.8352 \mathrm{E}+04$ & $1.7137 \mathrm{E}-05$ & & & \\
\hline Ir $190 \mathrm{~m}$ & 189.9606 & $1.200 \mathrm{E}+00$ & hour & $4.32 E+03$ & $1.3748 \mathrm{E}+07$ & $7.2740 \mathrm{E}-08$ & & & \\
\hline Ir 190m2 & 189.9606 & $3.250 \mathrm{E}+00$ & hour & $1.17 \mathrm{E}+04$ & $5.0761 \mathrm{E}+06$ & $1.9700 \mathrm{E}-07$ & & & \\
\hline Ir $191 \mathrm{~m}$ & 190.9606 & $4.940 \mathrm{E}+00$ & second & $4.94 E+00$ & $1.1959 \mathrm{E}+10$ & $8.3617 \mathrm{E}-11$ & & & \\
\hline Ir $191 \mathrm{~m} 2$ & 190.9606 & $5.500 \mathrm{E}+00$ & second & $5.50 \mathrm{E}+00$ & $1.0742 E+10$ & $9.3096 \mathrm{E}-11$ & & & \\
\hline Ir 192 & 191.9626 & $7.383 E+01$ & day & $6.38 \mathrm{E}+06$ & $9.2131 \mathrm{E}+03$ & $1.0854 \mathrm{E}-04$ & 0.006105 & & \\
\hline ir $192 \mathrm{~m}$ & 191.9626 & $1.450 \mathrm{E}+00$ & minute & $8.70 \mathrm{E}+01$ & $6.7552 E+08$ & $1.4803 \mathrm{E}-09$ & & & \\
\hline
\end{tabular}

WSRC-TR-99-00344

October, 1999

page 56 


\begin{tabular}{|c|c|c|c|c|c|c|c|c|c|}
\hline Isotope & $\begin{array}{l}\text { Atomic } \\
\text { Mass }\end{array}$ & Halflife & $\begin{array}{l}\text { Time } \\
\text { Units }\end{array}$ & $t_{1 / 2}, \sec$ & Curies/gram & Grams/ Curie & $\begin{array}{c}\text { Watts/CI } \\
\text { (Ref.3) }\end{array}$ & $\begin{array}{c}\text { Individual } \\
\text { Fission } \\
\text { Product Yield } \\
\text { (Ref.4) }\end{array}$ & $\begin{array}{c}\text { Cumulative } \\
\text { Fission Product } \\
\text { Yield (Ref.4) }\end{array}$ \\
\hline Ir $192 \mathrm{~m} 2$ & 191.9626 & $2.410 E+02$ & year & $7.61 \mathrm{E}+09$ & $7.7273 E+00$ & $1.2941 \mathrm{E}-01$ & & & \\
\hline If $193 \mathrm{~m}$ & 192.9629 & $1.053 E+01$ & day & $9.10 E+05$ & $6.4263 E+04$ & $1.5561 \mathrm{E}-05$ & & & \\
\hline If 194 & 193.9651 & $1.915 E+01$ & hour & $6.89 E+04$ & $8.4369 \mathrm{E}+05$ & $1.1853 \mathrm{E}-06$ & & & \\
\hline Ir 194m & 193.9651 & $3.185 \mathrm{E}-02$ & second & $3.19 \mathrm{E}-02$ & $1.8262 E+12$ & $5.4759 \mathrm{E}-13$ & & & \\
\hline Ir 194m2 & 193.9651 & $1.710 E+02$ & day & $1.48 \mathrm{E}+07$ & $3.9368 \mathrm{E}+03$ & $2.5401 \mathrm{E}-04$ & & & \\
\hline If 195 & 194.9660 & $2.500 E+00$ & hour & $9.00 \mathrm{E}+03$ & $6.4295 \mathrm{E}+06$ & $1.5553 \mathrm{E}-07$ & & & \\
\hline Ir $195 \mathrm{~m}$ & 194.9660 & $3.800 \mathrm{E}+00$ & hour & $1.37 \mathrm{E}+04$ & $4.2299 \mathrm{E}+06$ & $2.3641 \mathrm{E}-07$ & & & \\
\hline Ir 196 & 195.9684 & $5.200 \mathrm{E}+01$ & second & $5.20 E+01$ & $1.1071 \mathrm{E}+09$ & $9.0326 \mathrm{E}-10$ & & & \\
\hline Ir $196 \mathrm{~m}$ & 195.9684 & $1.400 E+00$ & hour & $5.04 E+03$ & $1.1422 \mathrm{E}+07$ & $8.7547 \mathrm{E}-08$ & & & \\
\hline If 197 & 196.9696 & $5.800 E+00$ & minute & $3.48 \mathrm{E}+02$ & $1.6459 \mathrm{E}+08$ & $6.0758 E-09$ & & & \\
\hline Ir $197 m$ & 196.9696 & $8.900 E+00$ & minute & $5.34 \mathrm{E}+02$ & $1.0726 \mathrm{E}+08$ & $9.3232 E-09$ & & & \\
\hline Ir 198 & 197.9723 & $8.000 E+00$ & second & $8.00 \mathrm{E}+00$ & $7.1233 E+09$ & $1.4038 E-10$ & & & \\
\hline Pt 169 & 168.9864 & $2.500 \mathrm{E}-03$ & second & $2.50 \mathrm{E}-03$ & $2.6705 E+13$ & $3.7447 E-14$ & & & \\
\hline Pt 170 & 169.9823 & $6.000 \mathrm{E}-03$ & second & $6.00 \mathrm{E}-03$ & $1.1062 \mathrm{E}+13$ & $9.0402 \mathrm{E}-14$ & & & \\
\hline$P+171$ & 170.9813 & $2.500 \mathrm{E}-02$ & second & $2.50 \mathrm{E}-02$ & $2.6393 \mathrm{E}+12$ & $3.7889 \mathrm{E}-13$ & & & \\
\hline Pt 172 & 171.9774 & $1.040 \mathrm{E}-01$ & second & $1.04 \mathrm{E}-01$ & $6.3077 \mathrm{E}+11$ & $1.5854 \mathrm{E}-12$ & & & \\
\hline Pt 173 & 172.9765 & $3.420 E-01$ & second & $3.42 \mathrm{E}-01$ & $1.9071 \mathrm{E}+11$ & $5.2437 E-12$ & & & \\
\hline Pt 174 & 173.9728 & $9.000 \mathrm{E}-01$ & second & $9.00 \mathrm{E}-01$ & $7.2053 \mathrm{E}+10$ & $1.3879 \mathrm{E}-11$ & & & \\
\hline Pt 175 & 174.9723 & $2.520 \mathrm{E}+00$ & second & $2.52 \mathrm{E}+00$ & $2.5586 \mathrm{E}+10$ & $3.9083 \mathrm{E}-11$ & & & \\
\hline Pt 176 & 175.9690 & $6.330 \mathrm{E}+00$ & second & $6.33 E+00$ & $1.0128 E+10$ & $9.8733 E-11$ & & & \\
\hline Pt 177 & 176.9685 & $1.100 \mathrm{E}+01$ & second & $1.10 \mathrm{E}+01$ & $5.7955 \mathrm{E}+09$ & $1.7255 \mathrm{E}-10$ & & & \\
\hline Pt 178 & 177.9657 & $2.110 E+01$ & second & $2.11 \mathrm{E}+01$ & $3.0044 E+09$ & $3.3285 \mathrm{E}-10$ & & & \\
\hline Pt 179 & 178.9655 & $2.120 E+01$ & second & $2.12 E+01$ & $2.9735 E+09$ & $3.3630 \mathrm{E}-10$ & & & \\
\hline Pt 180 & 179.9632 & $5.200 E+01$ & second & $5.20 \mathrm{E}+01$ & $1.2056 \mathrm{E}+09$ & $8.2949 E-10$ & & & \\
\hline Pt 181 & 180.9632 & $5.100 E+01$ & second & $5.10 E+01$ & $1.2224 \mathrm{E}+09$ & $8.1806 \mathrm{E}-10$ & & & \\
\hline Pt 182 & 181.9613 & $2.600 \mathrm{E}+00$ & minute & $1.56 \mathrm{E}+02$ & $3.9744 E+08$ & $2.5161 \mathrm{E}-09$ & & & \\
\hline Pt 183 & 182.9617 & $6.500 E+00$ & minute & $3.90 \mathrm{E}+02$ & $1.5811 \mathrm{E}+08$ & $6.3248 E-09$ & & & \\
\hline Pt $183 m$ & 182.9617 & $4.300 \mathrm{E}+01$ & second & $4.30 E+01$ & $1.4340 \mathrm{E}+09$ & $6.9735 E-10$ & & & \\
\hline Pt 184 & 183.9599 & $1.730 \mathrm{E}+01$ & minute & $1.04 \mathrm{E}+03$ & $5.9082 E+07$ & $1.6926 \mathrm{E}-08$ & & & \\
\hline Pt $184 m$ & 183.9599 & $1.010 \mathrm{E}-03$ & second & $1.01 \mathrm{E}-03$ & $6.0720 \mathrm{E}+13$ & $1.6469 \mathrm{E}-14$ & & & \\
\hline Pt 185 & 184.9608 & $7.090 E+01$ & minute & $4.25 E+03$ & $1.4338 \mathrm{E}+07$ & $6.9743 E-08$ & & & \\
\hline Pt $185 \mathrm{~m}$ & 184.9608 & $3.300 E+01$ & minute & $1.98 \mathrm{E}+03$ & $3.0806 E+07$ & $3.2461 \mathrm{E}-08$ & & & \\
\hline Pt 186 & 185.9594 & $2.000 \mathrm{E}+00$ & hour & $7.20 \mathrm{E}+03$ & $8.4261 E+06$ & $1.1868 \mathrm{E}-07$ & & & \\
\hline Pt 187 & 186.9606 & $2.350 E+00$ & hour & $8.46 E+03$ & $7.1327 E+06$ & $1.4020 \mathrm{E}-07$ & & & \\
\hline Pt 188 & 187.9594 & $1.020 E+01$ & day & $8.81 E+05$ & $6.8108 \mathrm{E}+04$ & $1.4683 \mathrm{E}-05$ & & & \\
\hline
\end{tabular}


Radionuclide Specific Data

\begin{tabular}{|c|c|c|c|c|c|c|c|c|c|}
\hline Isotope & $\begin{array}{l}\text { Atomic } \\
\text { Mass }\end{array}$ & Halflife & $\begin{array}{l}\text { Time } \\
\text { Units }\end{array}$ & $t_{1 / 2}$, sec & Curies/gram & Grams/ Curie & $\begin{array}{c}\text { Watts/Ci } \\
\text { (Ref.3) }\end{array}$ & $\begin{array}{c}\text { Individual }^{\dagger} \\
\text { Fission } \\
\text { Product Yield } \\
\text { (Ref.4) }\end{array}$ & $\begin{array}{l}\text { Cumulative }^{4} \\
\text { Fission Product } \\
\text { Yield (Ref.4) }\end{array}$ \\
\hline Pt 189 & 188.9608 & $1.087 E+01$ & hour & $3.91 E+04$ & $1.5257 \mathrm{E}+06$ & $6.5543 \mathrm{E}-07$ & & & \\
\hline Pt 190 & 189.9599 & $6.500 E+11$ & year & $2.05 E+19$ & 2.8952E-09 & $3.4539 E+08$ & & & \\
\hline Pt 191 & 190.9617 & $2.900 E+00$ & day & $2.51 E+05$ & $2.3579 E+05$ & $4.2411 \mathrm{E}-06$ & & & \\
\hline Pt 193 & 192.9630 & $5.000 E+01$ & year & $1.58 E+09$ & $3.7052 E+01$ & $2.6989 \mathrm{E}-02$ & & & \\
\hline Pt 193m & 192.9630 & $4.330 E+00$ & day & $3.74 E+05$ & $1.5628 \mathrm{E}+05$ & $6.3988 E-06$ & & & \\
\hline Pt $195 \mathrm{~m}$ & 194.9648 & $4.020 E+00$ & day & $3.47 E+05$ & $1.6660 \mathrm{E}+05$ & $6.0023 \mathrm{E}-06$ & & & \\
\hline Pt 197 & 196.9673 & $1.830 E+01$ & hour & $6.59 E+04$ & $8.6942 E+05$ & $1.1502 E-06$ & & & \\
\hline Pt $197 m$ & 196.9673 & $9.541 E+01$ & minute & $5.72 E+03$ & $1.0005 E+07$ & $9.9945 E-08$ & & & \\
\hline Pt 199 & 198.9706 & $3.080 E+01$ & minute & $1.85 E+03$ & $3.0682 E+07$ & $3.2592 E-08$ & & & \\
\hline Pt $199 m$ & 198.9706 & $1.360 E+01$ & second & $1.36 E+01$ & $4.1692 \mathrm{E}+09$ & $2.3986 E-10$ & & & \\
\hline Pt 200 & 199.9714 & $1.250 E+01$ & hour & $4.50 E+04$ & $1.2537 \mathrm{E}+06$ & $7.9763 \mathrm{E}-07$ & & & \\
\hline Pt 201 & 200.9745 & $2.500 E+00$ & minute & $1.50 E+02$ & $3.7423 E+08$ & $2.6721 E-09$ & & & \\
\hline$\widehat{A u 172}$ & 171.9901 & $4.000 \mathrm{E}-03$ & second & $4.00 \mathrm{E}-03$ & $1.6399 \mathrm{E}+13$ & $6.0980 E-14$ & & & \\
\hline Au 173 & 172.9864 & $5.900 \mathrm{E}-02$ & second & $5.90 \mathrm{E}-02$ & $1.1054 \mathrm{E}+12$ & $9.0466 \mathrm{E}-13$ & & & \\
\hline Au 174 & 173.9849 & $1.200 \mathrm{E}-01$ & second & $1.20 \mathrm{E}-01$ & $5.4036 E+11$ & $1.8506 E-12$ & & & \\
\hline Au 175 & 174.9816 & $2.000 E-01$ & second & $2.00 \mathrm{E}-01$ & $3.2237 \mathrm{E}+11$ & $3.1020 E-12$ & & & \\
\hline Au 176 & 175.9803 & $1.250 E+00$ & second & $1.25 E+00$ & $5.1286 E+10$ & $1.9498 \mathrm{E}-11$ & & & \\
\hline Au 177 & 176.9772 & $1.180 E+00$ & second & $1.18 E+00$ & $5.4023 \mathrm{E}+10$ & $1.8511 E-11$ & & & \\
\hline Au 178 & 177.9760 & $2.600 E+00$ & second & $2.60 E+00$ & $2.4380 \mathrm{E}+10$ & $4.1016 \mathrm{E}-11$ & & & \\
\hline Au 179 & 178.9734 & $7.100 E+00$ & second & $7.10 E+00$ & $8.8783 E+09$ & $1.1263 \mathrm{E}-10$ & & & \\
\hline$\widehat{A u ~ 180}$ & 179.9724 & $8.100 E+00$ & second & $8.10 E+00$ & $7.7390 \mathrm{E}+09$ & $1.2922 \mathrm{E}-10$ & & & \\
\hline Au 181 & 180.9699 & $1.140 E+01$ & second & $1.14 E+01$ & $5.4685 \mathrm{E}+09$ & $1.8287 E-10$ & & & \\
\hline Au 182 & 181.9696 & $2.100 E+01$ & second & $2.10 E+01$ & $2.9523 \mathrm{E}+09$ & $3.3872 E-10$ & & & \\
\hline Au 183 & 182.9676 & $4.200 E+01$ & second & $4.20 E+01$ & $1.4681 \mathrm{E}+09$ & $6.8116 \mathrm{E}-10$ & & & \\
\hline Au 184 & 183.9675 & $5.300 E+01$ & second & $5.30 \mathrm{E}+01$ & $1.1571 \mathrm{E}+09$ & $8.6425 \mathrm{E}-10$ & & & \\
\hline Au 185 & 184.9658 & $4.250 E+00$ & minute & $2.55 \mathrm{E}+02$ & $2.3919 \mathrm{E}+08$ & $4.1808 \mathrm{E}-09$ & & & \\
\hline Au $185 \mathrm{~m}$ & 184.9658 & $6.800 \mathrm{E}+00$ & minute & $4.08 E+02$ & $1.4949 \mathrm{E}+08$ & $6.6892 \mathrm{E}-09$ & & & \\
\hline Au 186 & 185.9660 & $1.070 E+01$ & minute & $6.42 \mathrm{E}+02$ & $9.4495 \mathrm{E}+07$ & $1.0583 \mathrm{E}-08$ & & & \\
\hline Au 187 & 186.9645 & $8.400 E+00$ & minute & $5.04 E+02$ & $1.1973 E+08$ & $8.3524 E-09$ & & & \\
\hline $\mathrm{Au} 187 \mathrm{~m}$ & 186.9645 & $2.300 \mathrm{E}+00$ & second & $2.30 \mathrm{E}+00$ & $2.6236 \mathrm{E}+10$ & $3.8116 \mathrm{E}-11$ & & & \\
\hline$A \cup 188$ & 187.9651 & $8.840 E+00$ & minute & $5.30 E+02$ & $1.1316 E+08$ & $8.8370 E-09$ & & & \\
\hline Au 189 & 188.9639 & $2.870 E+01$ & minute & $1.72 E+03$ & $3.4671 \mathrm{E}+07$ & $2.8843 E-08$ & & & \\
\hline Au $189 \mathrm{~m}$ & 188.9639 & $4.590 \mathrm{E}+00$ & minute & $2.75 \mathrm{E}+02$ & $2.1679 \mathrm{E}+08$ & $4.6128 \mathrm{E}-09$ & & & \\
\hline Au 190 & 189.9647 & $4.280 \mathrm{E}+01$ & minute & $2.57 E+03$ & $2.3126 \mathrm{E}+07$ & $4.3241 \mathrm{E}-08$ & & & \\
\hline Au 190m & 189.9647 & $1.250 \mathrm{E}-01$ & second & $1.25 \mathrm{E}-01$ & $4.7511 \mathrm{E}+11$ & $2.1048 E-12$ & & & \\
\hline
\end{tabular}

WSRC-TR-99-00344

October, 1999

page 58 
Radionuclide Specific Data

\begin{tabular}{|c|c|c|c|c|c|c|c|c|c|}
\hline Isotope & $\begin{array}{c}\text { Atomic } \\
\text { Mass }\end{array}$ & Halflife & $\begin{array}{l}\text { Time } \\
\text { Units }\end{array}$ & $t_{1 / 2}, \sec$ & Curies/gram & Grams/ Curie & $\begin{array}{c}\text { Watts/Ci } \\
\text { (Ref.3) }\end{array}$ & $\begin{array}{l}\text { Individual' } \\
\text { Fission } \\
\text { Product Yield } \\
\text { (Ref.4) }\end{array}$ & $\begin{array}{c}\text { Cumulative } \\
\text { Fission Product } \\
\text { Yield (Ref.4) }\end{array}$ \\
\hline Au 191 & 190.9636 & $3.180 E+00$ & hour & $1.14 E+04$ & $5.1606 \mathrm{E}+06$ & $1.9378 \mathrm{E}-07$ & & & \\
\hline Au 191m & 190.9636 & $9.200 \mathrm{E}-01$ & second & $9.20 \mathrm{E}-01$ & $6.4215 \mathrm{E}+10$ & $1.5573 \mathrm{E}-11$ & & & \\
\hline Au 192 & 191.9648 & $4.940 E+00$ & hour & $1.78 E+04$ & $3.3046 E+06$ & $3.0260 \mathrm{E}-07$ & & & \\
\hline Au $192 m$ & 191.9648 & $1.600 \mathrm{E}-01$ & second & $1.60 \mathrm{E}-01$ & $3.6731 \mathrm{E}+11$ & $2.7225 \mathrm{E}-12$ & & & \\
\hline Au 193 & 192.9641 & $1.765 E+01$ & hour & $6.35 E+04$ & $9.2014 \mathrm{E}+05$ & $1.0868 \mathrm{E}-06$ & & & \\
\hline Au 193m & 192.9641 & $3.900 E+00$ & second & $3.90 E+00$ & $1.4991 E+10$ & $6.6706 \mathrm{E}-11$ & & & \\
\hline Au 194 & 193.9653 & $3.802 E+01$ & hour & $1.37 E+05$ & $4.2495 \mathrm{E}+05$ & $2.3532 \mathrm{E}-06$ & & & \\
\hline Au $194 \mathrm{~m}$ & 193.9653 & $6.000 \mathrm{E}-01$ & second & $6.00 \mathrm{E}-01$ & $9.6940 \mathrm{E}+10$ & $1.0316 \mathrm{E}-11$ & & & \\
\hline$\overline{A u ~ 194 \mathrm{~m}^{2}}$ & 193.9653 & $4.200 \mathrm{E}-01$ & second & $4.20 \mathrm{E}-01$ & $1.3849 E+11$ & $7.2210 \mathrm{E}-12$ & & & \\
\hline Au 195 & 194.9650 & $1.861 E+02$ & day & $1.61 E+07$ & $3.5990 E+03$ & $2.7785 \mathrm{E}-04$ & & & \\
\hline Au 195m & 194.9650 & $3.050 E+01$ & second & $3.05 E+01$ & $1.8972 \mathrm{E}+09$ & $5.2708 \mathrm{E}-10$ & & & \\
\hline Au 196 & 195.9666 & $6.183 E+00$ & day & $5.34 E+05$ & $1.0777 \mathrm{E}+05$ & $9.2794 \mathrm{E}-06$ & & & \\
\hline $\mathrm{Au} 196 \mathrm{~m}$ & 195.9666 & $8.100 E+00$ & second & $8.10 E+00$ & $7.1074 \mathrm{E}+09$ & $1.4070 \mathrm{E}-10$ & & & \\
\hline$\overline{A u 196 \mathrm{~m}^{2}}$ & 195.9666 & $9.700 E+00$ & hour & $3.49 E+04$ & $1.6486 E+06$ & $6.0657 \mathrm{E}-07$ & & & \\
\hline Au $197 m$ & 196.9666 & $7.730 E+00$ & second & $7.73 E+00$ & $7.4098 E+09$ & $1.3496 \mathrm{E}-10$ & & & \\
\hline Au 198 & 197.9682 & $2.695 \mathrm{E}+00$ & day & $2.33 E+05$ & $2.4473 E+05$ & $4.0862 \mathrm{E}-06$ & & & \\
\hline \begin{tabular}{|l} 
Au $198 m$ \\
\end{tabular} & 197.9682 & $2.300 E+00$ & day & $1.99 E+05$ & $2.8677 \mathrm{E}+05$ & $3.4871 E-06$ & & & \\
\hline Au 199 & 198.9687 & $3.139 E+00$ & day & $2.71 E+05$ & $2.0907 E+05$ & 4.7831E-06 & & & \\
\hline Au 200 & 199.9707 & $4.840 E+01$ & minute & $2.90 \mathrm{E}+03$ & $1.9427 E+07$ & $5.1474 \mathrm{E}-08$ & & & \\
\hline $\mathrm{Au} 200 \mathrm{~m}$ & 199.9707 & $1.870 E+01$ & hour & $6.73 E+04$ & $8.3804 E+05$ & $1.1933 \mathrm{E}-06$ & & & \\
\hline Au 201 & 200.9716 & $2.600 E+01$ & minute & $1.56 \mathrm{E}+03$ & $3.5985 E+07$ & $2.7790 \mathrm{E}-08$ & & & \\
\hline Au 202 & 201.9738 & $2.880 E+01$ & second & $2.88 E+01$ & $1.9395 \mathrm{E}+09$ & $5.1560 \mathrm{E}-10$ & & & \\
\hline $\mathrm{Au} 203$ & 202.9751 & $5.300 E+01$ & second & $5.30 \mathrm{E}+01$ & $1.0487 \mathrm{E}+09$ & $9.5355 \mathrm{E}-10$ & & & \\
\hline Au 204 & 203.9777 & $3.980 \mathrm{E}+01$ & second & $3.98 E+01$ & $1.3897 E+09$ & $7.1960 \mathrm{E}-10$ & & & \\
\hline $\operatorname{Hg~} 175$ & 174.9914 & $2.000 \mathrm{E}-02$ & second & $2.00 \mathrm{E}-02$ & $3.2235 \mathrm{E}+12$ & $3.1022 \mathrm{E}-13$ & & & \\
\hline $\mathrm{Hg} \mathrm{176}$ & 175.9874 & $3.400 \mathrm{E}-02$ & second & $3.40 \mathrm{E}-02$ & $1.8855 E+12$ & $5.3038 \mathrm{E}-13$ & & & \\
\hline $\mathrm{Hg} 177$ & 176.9772 & $1.300 \mathrm{E}-01$ & second & $1.30 E-01$ & $4.9036 E+11$ & $2.0393 \mathrm{E}-12$ & & & \\
\hline $\mathrm{Hg} \mathrm{178}$ & 177.9825 & $2.540 \mathrm{E}-01$ & second & $2.54 E-01$ & $2.4955 \mathrm{E}+11$ & $4.0071 \mathrm{E}-12$ & & & \\
\hline $\operatorname{Hg} 179$ & 178.9818 & $1.090 \mathrm{E}+00$ & second & $1.09 E+00$ & $5.7828 \mathrm{E}+10$ & $1.7293 \mathrm{E}-11$ & & & \\
\hline $\mathrm{Hg} 180$ & 179.9783 & $2.800 \mathrm{E}+00$ & second & $2.80 E+00$ & $2.2387 \mathrm{E}+10$ & $4.4669 \mathrm{E}-11$ & & & \\
\hline Hg 181 & 180.9778 & $3.600 \mathrm{E}+00$ & second & $3.60 \mathrm{E}+00$ & $1.7316 E+10$ & $5.7750 \mathrm{E}-11$ & & & \\
\hline $\mathrm{Hg} 182$ & 181.9748 & $1.083 E+01$ & second & $1.08 \mathrm{E}+01$ & $5.7245 E+09$ & $1.7469 \mathrm{E}-10$ & & & \\
\hline $\mathrm{Hg} 183$ & 182.9746 & $8.800 E+00$ & second & $8.80 E+00$ & $7.0065 E+09$ & $1.4272 \mathrm{E}-10$ & & & \\
\hline $\mathrm{Hg} 184$ & 183.9719 & $3.060 \mathrm{E}+01$ & second & $.3 .06 E+01$ & $2.0040 E+09$ & $4.9900 \mathrm{E}-10$ & & & \\
\hline Hg 185 & 184.9720 & $4.910 E+01$ & second & $4.91 \mathrm{E}+01$ & $1.2422 \mathrm{E}+09$ & $8.0503 \mathrm{E}-10$ & & & \\
\hline
\end{tabular}

WSRC-TR-99-00344

October, 1999

page 59 


\begin{tabular}{|c|c|c|c|c|c|c|c|c|c|}
\hline Isotope & $\begin{array}{l}\text { Atomic } \\
\text { Mass }\end{array}$ & Halflife & $\begin{array}{l}\text { Time } \\
\text { Units }\end{array}$ & $t_{12}, \sec$ & Curies/ gram & Grams/ Curie & $\begin{array}{c}\text { Watts/Ci } \\
\text { (Ref.3) }\end{array}$ & $\begin{array}{c}\text { Individual' } \\
\text { Fission } \\
\text { Product Yield } \\
\text { (Ref.4) }\end{array}$ & $\begin{array}{c}\text { Cumulative }^{4} \\
\text { Fission Product } \\
\text { Yield (Ref.4) }\end{array}$ \\
\hline $\mathrm{Hg} 185 \mathrm{~m}$ & 184.9720 & $2.160 E+01$ & second & $2.16 E+01$ & $2.8237 E+09$ & $3.5415 \mathrm{E}-10$ & & & \\
\hline $\mathrm{Hg} 186$ & 185.9695 & $1.380 E+00$ & minute & $8.28 E+01$ & $7.3266 \mathrm{E}+08$ & $1.3649 \mathrm{E}-09$ & & & \\
\hline $\mathrm{Hg} 187$ & 186.9698 & $2.400 E+00$ & minute & $1.44 E+02$ & $4.1903 \mathrm{E}+08$ & $2.3865 E-09$ & & & \\
\hline $\mathrm{Hg} 187 \mathrm{~m}$ & 186.9698 & $1.900 E+00$ & minute & $1.14 E+02$ & $5.2930 \mathrm{E}+08$ & $1.8893 \mathrm{E}-09$ & & & \\
\hline $\mathrm{Hg} 188$ & 187.9676 & $3.250 E+00$ & minute & $1.95 E+02$ & $3.0779 E+08$ & $3.2489 \mathrm{E}-09$ & & & \\
\hline $\mathrm{Hg} 189$ & 188.9681 & $7.600 E+00$ & minute & $4.56 E+02$ & $1.3093 E+08$ & $7.6380 \mathrm{E}-09$ & & & \\
\hline $\mathrm{Hg} 189 \mathrm{~m}$ & 188.9681 & $8.600 E+00$ & minute & $5.16 \mathrm{E}+02$ & $1.1570 E+08$ & $8.6429 \mathrm{E}-09$ & & & \\
\hline $\mathrm{Hg} 190$ & 189.9663 & $2.000 \mathrm{E}+01$ & minute & $1.20 \mathrm{E}+03$ & $4.9490 \mathrm{E}+07$ & $2.0206 \mathrm{E}-08$ & & & \\
\hline $\mathrm{Hg} 191$ & 190.9671 & $4.900 \mathrm{E}+01$ & minute & $2.94 E+03$ & $2.0094 E+07$ & $4.9766 E-08$ & & & \\
\hline $\mathrm{Hg} 191 \mathrm{~m}$ & 190.9671 & $5.080 E+01$ & minute & $3.05 E+03$ & $1.9382 E+07$ & $5.1594 \mathrm{E}-08$ & & & \\
\hline $\mathrm{Hg} 192$ & 191.9656 & $4.850 \mathrm{E}+00$ & hour & $1.75 \mathrm{E}+04$ & $3.3660 \mathrm{E}+06$ & 2.9709E-07 & & & \\
\hline $\mathrm{Hg} 193$ & 192.9666 & $3.800 E+00$ & hour & $1.37 E+04$ & $4.2737 E+06$ & $2.3399 \mathrm{E}-07$ & & & \\
\hline $\mathrm{Hg} 193 \mathrm{~m}$ & 192.9666 & $1.180 E+01$ & hour & $4.25 E+04$ & $1.3763 E+06$ & $7.2659 \mathrm{E}-07$ & & & \\
\hline $\mathrm{Hg} 194$ & 193.9654 & $4.440 \mathrm{E}+02$ & year & $1.40 E+10$ & $4.1510 E+00$ & $2.4091 \mathrm{E}-01$ & & & \\
\hline $\mathrm{Hg} 195$ & 194.9666 & $9.900 E+00$ & hour & $3.56 E+04$ & $1.6236 E+06$ & $6.1592 E-07$ & & & \\
\hline $\mathrm{Hg} 195 \mathrm{~m}$ & 194.9666 & $4.160 E+01$ & hour & $1.50 E+05$ & $3.8639 E+05$ & $2.5881 \mathrm{E}-06$ & & & \\
\hline $\mathrm{Hg} 197$ & 196.9672 & $6.414 E+01$ & hour & $2.31 \mathrm{E}+05$ & $2.4806 E+05$ & $4.0313 \mathrm{E}-06$ & & & \\
\hline $\mathrm{Hg} 197 \mathrm{~m}$ & 196.9672 & $2.380 E+01$ & hour & $8.57 \mathrm{E}+04$ & $6.6850 \mathrm{E}+05$ & $1.4959 \mathrm{E}-06$ & & & \\
\hline $\mathrm{Hg} \mathrm{199m}$ & 198.9683 & $4.260 E+01$ & minute & $2.56 E+03$ & $2.2184 E+07$ & $4.5078 E-08$ & & & \\
\hline $\mathrm{Hg} 203$ & 202.9729 & $4.661 E+01$ & day & $4.03 E+06$ & $1.3802 E+04$ & $7.2456 \mathrm{E}-05$ & & & \\
\hline $\mathrm{Hg} 205$ & 204.9761 & $5.200 E+00$ & minute & $3.12 E+02$ & $1.7641 \mathrm{E}+08$ & $5.6687 \mathrm{E}-09$ & & & \\
\hline $\mathrm{Hg} 206$ & 205.9775 & $8.150 E+00$ & minute & $4.89 E+02$ & $1.1201 \mathrm{E}+08$ & $8.9280 \mathrm{E}-09$ & & & \\
\hline $\mathrm{Hg} 207$ & 206.9826 & $2.900 E+00$ & minute & $1.74 E+02$ & $3.1325 \mathrm{E}+08$ & $3.1923 E-09$ & & & \\
\hline TI 179 & 178.9915 & $1.600 \mathrm{E}-01$ & second & $1.60 \mathrm{E}-01$ & $3.9393 E+11$ & $2.5385 E-12$ & & & \\
\hline $\mathrm{TI} 179 \mathrm{~m}$ & 178.9915 & $1.400 \mathrm{E}-03$ & second & $1.40 E-03$ & $4.5021 \mathrm{E}+13$ & $2.2212 \mathrm{E}-14$ & & & \\
\hline TI 180 & 179.9902 & $7.000 \mathrm{E}-01$ & second & $7.00 \mathrm{E}-01$ & $8.9543 E+10$ & $1.1168 \mathrm{E}-11$ & & & \\
\hline TI 182 & 181.9856 & $3.100 E+00$ & second & $3.10 E+00$ & $1.9998 E+10$ & $5.0006 \mathrm{E}-11$ & & & \\
\hline TI 183 & 182.9827 & $6.900 E+00$ & second & $6.90 \mathrm{E}+00$ & $8.9355 E+09$ & $1.1191 \mathrm{E}-10$ & & & \\
\hline $\mathrm{TI} 183 \mathrm{~m}$ & 182.9827 & $6.000 E-02$ & second & $6.00 \mathrm{E}-02$ & $1.0276 \mathrm{E}+12$ & $9.7316 \mathrm{E}-13$ & & & \\
\hline TI 184 & 183.9818 & $1.100 \mathrm{E}+01$ & second & $1.10 E+01$ & $5.5745 E+09$ & $1.7939 \mathrm{E}-10$ & & & \\
\hline TI 185 & 184.9791 & $1.950 E+01$ & second & $1.95 \mathrm{E}+01$ & $3.1277 E+09$ & $3.1973 \mathrm{E}-10$ & & & \\
\hline $\mathrm{TI} 185 \mathrm{~m}$ & 184.9791 & $1.830 E+00$ & second & $1.83 \mathrm{E}+00$ & $3.3328 \mathrm{E}+10$ & $3.0005 E-11$ & & & \\
\hline TI 186 & 185.9785 & $2.750 E+01$ & second & $2.75 \mathrm{E}+01$ & $2.2059 \mathrm{E}+09$ & $4.5333 \mathrm{E}-10$ & & & \\
\hline TI 186m & 185.9785 & $2.900 E+00$ & second & $2.90 \mathrm{E}+00$ & $2.0918 \mathrm{E}+10$ & $4.7806 \mathrm{E}-11$ & & & \\
\hline TI 187 & 186.9762 & $5.100 E+01$ & second & $5.10 \mathrm{E}+01$ & $1.1831 E+09$ & $8.4524 \mathrm{E}-10$ & & & \\
\hline
\end{tabular}

WSRC-TR-99-00344

October, 1999

page 60 
Radionuclide Specific Data

\begin{tabular}{|c|c|c|c|c|c|c|c|c|c|}
\hline Isotope & $\begin{array}{l}\text { Atomic } \\
\text { Mass }\end{array}$ & Halflife & $\begin{array}{l}\text { Time } \\
\text { Units }\end{array}$ & $t_{1 / 2}, \sec$ & Curies/gram & Grams/Curie & $\begin{array}{c}\text { Watts/Ci } \\
\text { (Ref.3) }\end{array}$ & $\begin{array}{c}\text { Individual }^{4} \\
\text { Fission } \\
\text { Product Yield } \\
\text { (Ref.4) }\end{array}$ & \begin{tabular}{|c|} 
Cumulative $^{1}$ \\
Fission Product \\
Yield (Ref.4)
\end{tabular} \\
\hline TI $187 m$ & 186.9762 & $1.560 \mathrm{E}+01$ & second & $1.56 \mathrm{E}+01$ & $3.8678 \mathrm{E}+09$ & $2.5854 \mathrm{E}-10$ & & & \\
\hline T1 188 & 187.9759 & $7.100 E+01$ & second & $7.10 E+01$ & $8.4531 E+08$ & $1.1830 \mathrm{E}-09$ & & & \\
\hline T/ $188 \mathrm{~m}$ & 187.9759 & $7.100 E+01$ & second & $7.10 E+01$ & $8.4531 E+08$ & $1.1830 \mathrm{E}-09$ & & & \\
\hline TI 189 & 188.9737 & $2.300 \mathrm{E}+00$ & minute & $1.38 \mathrm{E}+02$ & $4.3261 \mathrm{E}+08$ & $2.3116 \mathrm{E}-09$ & & & \\
\hline TI $189 \mathrm{~m}$ & 188.9737 & $1.400 \mathrm{E}+00$ & minute & $8.40 E+01$ & $7.1072 E+08$ & $1.4070 \mathrm{E}-09$ & & & \\
\hline$T 1190$ & 189.9738 & $2.600 E+00$ & minute & $1.56 E+02$ & $3.8068 \mathrm{E}+08$ & $2.6269 \mathrm{E}-09$ & & & \\
\hline TI 190m & 189.9738 & $3.700 E+00$ & minute & $2.22 E+02$ & $2.6750 E+08$ & $3.7383 E-09$ & & & \\
\hline TI191m & 190.9719 & $5.220 E+00$ & minute & $3.13 E+02$ & $1.8862 \mathrm{E}+08$ & $5.3017 \mathrm{E}-09$ & & & \\
\hline TI 192 & 191.9721 & $9.600 \mathrm{E}+00$ & minute & $5.76 \mathrm{E}+02$ & $1.0203 E+08$ & $9.8013 \mathrm{E}-09$ & & & \\
\hline TI 192m & 191.9721 & $1.080 E+01$ & minute & $6.48 E+02$ & $9.0691 \mathrm{E}+07$ & $1.1026 \mathrm{E}-08$ & & & \\
\hline TI 193 & 192.9705 & $2.160 \mathrm{E}+01$ & minute & $1.30 \mathrm{E}+03$ & $4.5111 E+07$ & $2.2168 E-08$ & & & \\
\hline TI 193m & 192.9705 & $2.110 E+00$ & minute & $1.27 E+02$ & $4.6180 \mathrm{E}+08$ & $2.1655 E-09$ & & & \\
\hline TI 194 & 193.9711 & $3.300 E+01$ & minute & $1.98 E+03$ & $2.9375 E+07$ & $3.4043 E-08$ & & & \\
\hline$T I 194 m$ & 193.9711 & $3.280 E+01$ & minute & $1.97 \mathrm{E}+03$ & $2.9554 E+07$ & $3.3837 \mathrm{E}-08$ & & & \\
\hline TI195 & 194.9697 & $1.160 E+00$ & hour & $4.18 \mathrm{E}+03$ & $1.3856 \mathrm{E}+07$ & $7.2169 \mathrm{E}-08$ & & & \\
\hline TI 195m & 194.9697 & $3.600 \mathrm{E}+00$ & second & $3.60 \mathrm{E}+00$ & $1.6073 \mathrm{E}+10$ & $6.2215 E-11$ & & & \\
\hline TI 196 & 195.9705 & $1.840 E+00$ & hour & $6.62 E+03$ & $8.6909 \mathrm{E}+06$ & $1.1506 \mathrm{E}-07$ & & & \\
\hline TI 196m & 195.9705 & $1.410 E+00$ & hour & $5.08 \mathrm{E}+03$ & $1.1341 \mathrm{E}+07$ & $8.8173 \mathrm{E}-08$ & & & \\
\hline TI 197 & 196.9695 & $2.840 E+00$ & hour & $1.02 E+04$ & $5.6022 \mathrm{E}+06$ & $1.7850 \mathrm{E}-07$ & & & \\
\hline TI 197m & 196.9695 & $5.400 \mathrm{E}-01$ & second & $5.40 \mathrm{E}-01$ & $1.0607 \mathrm{E}+11$ & $9.4279 E-12$ & & & \\
\hline TI 198 & 197.9405 & $5.300 \mathrm{E}+00$ & hour & $1.91 E+04$ & $2.9872 \mathrm{E}+06$ & $3.3476 \mathrm{E}-07$ & & & \\
\hline TI 198m & 197.9405 & $1.870 E+00$ & hour & $6.73 E+03$ & $8.4664 E+06$ & 1.1811E-07 & & & \\
\hline TI 198m2 & 197.9405 & $3.210 \mathrm{E}-02$ & second & $3.21 \mathrm{E}-02$ & $1.7756 E+12$ & $5.6320 \mathrm{E}-13$ & & & \\
\hline TI 199 & 198.9698 & $7.420 \mathrm{E}+00$ & hour & $2.67 E+04$ & $2.1227 E+06$ & $4.7110 \mathrm{E}-07$ & & & \\
\hline TI199m & 198.9698 & $2.840 \mathrm{E}-02$ & second & $2.84 \mathrm{E}-02$ & $1.9965 \mathrm{E}+12$ & $5.0087 \mathrm{E}-13$ & & & \\
\hline$T 1200$ & 199.9709 & $2.610 E+01$ & hour & $9.40 \mathrm{E}+04$ & $6.0044 E+05$ & $1.6655 \mathrm{E}-06$ & & & \\
\hline $\mathrm{Tl} 200 \mathrm{~m}$ & 199.9709 & $3.430 \mathrm{E}-02$ & second & $3.43 \mathrm{E}-02$ & $1.6448 E+12$ & $6.0797 \mathrm{E}-13$ & & & \\
\hline TI 201 & 200.9708 & $7.291 E+01$ & hour & $2.62 E+05$ & $2.1387 E+05$ & $4.6758 \mathrm{E}-06$ & 0.000830 & & \\
\hline$T 1201 \mathrm{~m}$ & 200.9708 & $2.035 \mathrm{E}-03$ & second & $2.04 \mathrm{E}-03$ & $2.7585 E+13$ & $3.6251 \mathrm{E}-14$ & & & \\
\hline$T 11202$ & 201.9721 & $1.223 \mathrm{E}+01$ & day & $1.06 \mathrm{E}+06$ & $5.2862 \mathrm{E}+04$ & $1.8917 \mathrm{E}-05$ & & & \\
\hline$\overline{T 1204}$ & 203.9738 & $3.780 \mathrm{E}+00$ & year & $1.19 E+08$ & $4.6365 \mathrm{E}+02$ & $2.1568 \mathrm{E}-03$ & & & \\
\hline$T 1206$ & 205.9761 & $4.199 E+00$ & minute & $2.52 E+02$ & $2.1740 E+08$ & $4.5998 \mathrm{E}-09$ & & & \\
\hline $\mathrm{T} / 206 \mathrm{~m}$ & 205.9761 & $3.740 E+00$ & minute & $2.24 E+02$ & $2.4408 E+08$ & 4.0970E-09 & & & \\
\hline T1207 & 206.9774 & $4.770 E+00$ & minute & $2.86 \mathrm{E}+02$ & $1.9045 E+08$ & $5.2507 \mathrm{E}-09$ & 0.002930 & & \\
\hline$T / 207 \mathrm{~m}$ & 206.9774 & $1.330 \mathrm{E}+00$ & second & $1.33 E+00$ & $4.0983 E+10$ & $2.4400 \mathrm{E}-11$ & & & \\
\hline
\end{tabular}

WSRC-TR-99-00344

October, 1999

page 61 


\begin{tabular}{|c|c|c|c|c|c|c|c|c|c|}
\hline Isotope & $\begin{array}{l}\text { Atomic } \\
\text { Mass }\end{array}$ & Halflife & $\begin{array}{l}\text { Time } \\
\text { Units }\end{array}$ & $t_{1 / 2}, \mathrm{sec}$ & Curies/ gram & Grams/ Curie & $\begin{array}{l}\text { Watts/Ci } \\
\text { (Ref.3) }\end{array}$ & $\begin{array}{c}\text { Individual }^{1} \\
\text { Fission } \\
\text { Product Yield } \\
\text { (Ref.4) }\end{array}$ & $\begin{array}{c}\text { Cumulative' } \\
\text { Fission Product } \\
\text { Yield (Ref.4) }\end{array}$ \\
\hline $\mathrm{TI} 208$ & 207.9820 & $3.053 E+00$ & minute & $1.83 E+02$ & $2.9612 E+08$ & $3.3770 \mathrm{E}-09$ & 0.023540 & & \\
\hline$T 1209$ & 208.9853 & $2.200 E+00$ & minute & $1.32 \mathrm{E}+02$ & $4.0897 E+08$ & $2.4452 E-09$ & & & \\
\hline$T / 210$ & 209.9901 & $1.300 E+00$ & minute & $7.80 \mathrm{E}+01$ & $6.8878 E+08$ & $1.4518 \mathrm{E}-09$ & & & \\
\hline $\mathrm{Pb} 181$ & 180.9967 & $5.000 \mathrm{E}-02$ & second & $5.00 \mathrm{E}-02$ & $1.2466 \mathrm{E}+12$ & $8.0217 \mathrm{E}-13$ & & & \\
\hline $\mathrm{Pb} 182$ & 181.9927 & $5.500 \mathrm{E}-02$ & second & $5.50 \mathrm{E}-02$ & $1.1271 E+12$ & $8.8724 \mathrm{E}-13$ & & & \\
\hline $\mathrm{Pb} 183$ & 182.9919 & $3.000 \mathrm{E}-01$ & second & $3.00 \mathrm{E}-01$ & $2.0551 E+11$ & $4.8661 \mathrm{E}-12$ & & & \\
\hline $\mathrm{Pb} 184$ & 183.9882 & $5.500 \mathrm{E}-01$ & second & $5.50 \mathrm{E}-01$ & $1.1149 E+11$ & $8.9697 E-12$ & & & \\
\hline $\mathrm{Pb} 185$ & 184.9876 & $4.100 E+00$ & second & $4.10 E+00$ & $1.4875 \mathrm{E}+10$ & $6.7228 \mathrm{E}-11$ & & & \\
\hline $\mathrm{Pb} 186$ & 185.9843 & $4.700 E+00$ & second & $4.70 \mathrm{E}+00$ & $1.2906 E+10$ & $7.7481 \mathrm{E}-11$ & & & \\
\hline $\mathrm{Pb} 187$ & 186.9840 & $1.830 E+01$ & second & $1.83 E+01$ & $3.2970 E+09$ & $3.0330 \mathrm{E}-10$ & & & \\
\hline $\mathrm{Pb} 187 \mathrm{~m}$ & 186.9840 & $1.520 \mathrm{E}+01$ & second & $1.52 E+01$ & $3.9694 E+09$ & $2.5193 \mathrm{E}-10$ & & & \\
\hline $\mathrm{Pb} 188$ & 187.9759 & $2.420 E+01$ & second & $2.42 E+01$ & $2.4800 \mathrm{E}+09$ & $4.0322 \mathrm{E}-10$ & & & \\
\hline $\mathrm{Pb} 189$ & 188.9809 & $5.100 E+01$ & second & $5.10 \mathrm{E}+01$ & $1.1705 E+09$ & $8.5430 \mathrm{E}-10$ & & & \\
\hline $\mathrm{Pb} \mathrm{190}$ & 189.9782 & $1.200 E+00$ & minute & $7.20 \mathrm{E}+01$ & $8.2478 E+08$ & $1.2124 \mathrm{E}-09$ & & & \\
\hline $\mathrm{Pb} 191$ & 190.9782 & $1.330 E+00$ & minute & $7.98 \mathrm{E}+01$ & $7.4027 E+08$ & $1.3509 \mathrm{E}-09$ & & & \\
\hline $\mathrm{Pb} 191 \mathrm{~m}$ & 190.9782 & $2.180 E+00$ & minute & $1.31 E+02$ & $4.5163 E+08$ & $2.2142 \mathrm{E}-09$ & & & \\
\hline $\mathrm{Pb} 192$ & 191.9758 & $3.500 \mathrm{E}+00$ & minute & $2.10 \mathrm{E}+02$ & $2.7984 E+08$ & 3.5735E-09 & & & \\
\hline $\mathrm{Pb} 193 \mathrm{~m}$ & 192.9761 & $5.800 E+00$ & minute & $3.48 \mathrm{E}+02$ & $1.6799 \mathrm{E}+08$ & $5.9526 \mathrm{E}-09$ & & & \\
\hline $\mathrm{Pb} 194$ & 193.9740 & $1.200 E+01$ & minute & $7.20 E+02$ & $8.0779 E+07$ & 1.2379E-08 & & & \\
\hline $\mathrm{Pb} 195$ & 194.9745 & $1.500 E+01$ & minute & $9.00 \mathrm{E}+02$ & $6.4292 \mathrm{E}+07$ & $1.5554 \mathrm{E}-08$ & & & \\
\hline $\mathrm{Pb} 195 \mathrm{~m}$ & 194.9745 & $1.500 \mathrm{E}+01$ & minute & $9.00 \mathrm{E}+02$ & $6.4292 \mathrm{E}+07$ & $1.5554 E-08$ & & & \\
\hline $\mathrm{Pb} 196$ & 195.9727 & $3.700 \mathrm{E}+01$ & minute & $2.22 E+03$ & $2.5932 \mathrm{E}+07$ & $3.8563 \mathrm{E}-08$ & & & \\
\hline $\mathrm{Pb} 197$ & 196.9734 & $8.000 E+00$ & minute & $4.80 \mathrm{E}+02$ & $1.1932 E+08$ & $8.3805 E-09$ & & & \\
\hline $\mathrm{Pb} 197 \mathrm{~m}$ & 196.9734 & $4.300 E+01$ & minute & $2.58 \mathrm{E}+03$ & $2.2200 E+07$ & 4.5045E-08 & & & \\
\hline $\mathrm{Pb} 198$ & 197.9720 & $2.400 E+00$ & hour & $8.64 \mathrm{E}+03$ & $6.5957 \mathrm{E}+06$ & $1.5161 \mathrm{E}-07$ & & & \\
\hline Pb 199 & 198.9729 & $1.500 E+00$ & hour & $5.40 \mathrm{E}+03$ & $1.0500 \mathrm{E}+07$ & $9.5238 \mathrm{E}-08$ & & & \\
\hline $\mathrm{Pb} 199 \mathrm{~m}$ & 198.9729 & $1.220 E+01$ & minute & $7.32 E+02$ & $7.7459 \mathrm{E}+07$ & 1.2910E-08 & & & \\
\hline $\mathrm{Pb} 200$ & 199.9718 & $2.150 \mathrm{E}+01$ & hour & $7.74 \mathrm{E}+04$ & $7.2890 \mathrm{E}+05$ & $1.3719 \mathrm{E}-06$ & & & \\
\hline $\mathrm{Pb} 201$ & 200.9728 & $9.330 \mathrm{E}+00$ & hour & $3.36 E+04$ & $1.6713 E+06$ & $5.9834 \mathrm{E}-07$ & & & \\
\hline $\mathrm{Pb} 201 \mathrm{~m}$ & 200.9728 & $6.100 \mathrm{E}+01$ & second & $6.10 \mathrm{E}+01$ & $9.2026 \mathrm{E}+08$ & $1.0867 \mathrm{E}-09$ & & & \\
\hline $\mathrm{Pb} 202$ & 201.9721 & $5.250 \mathrm{E}+04$ & year & $1.66 \mathrm{E}+12$ & $3.3714 \mathrm{E}-02$ & $2.9661 \mathrm{E}+01$ & & & \\
\hline $\mathrm{Pb} \mathrm{202m}$ & 201.9721 & $3.530 \mathrm{E}+00$ & hour & $1.27 E+04$ & $4.3955 \mathrm{E}+06$ & $2.2751 \mathrm{E}-07$ & & & \\
\hline $\mathrm{Pb} 203$ & 202.9734 & $5.187 \mathrm{E}+01$ & hour & $1.87 E+05$ & $2.9764 E+05$ & 3.3597E-06 & & & \\
\hline $\mathrm{Pb} 203 \mathrm{~m}$ & 202.9734 & $6.300 \mathrm{E}+00$ & second & $6.30 \mathrm{E}+00$ & $8.8226 \mathrm{E}+09$ & $1.1335 \mathrm{E}-10$ & & & \\
\hline $\mathrm{Pb} \mathrm{203m2}$ & 202.9734 & $4.800 \mathrm{E}-01$ & second & $4.80 \mathrm{E}-01$ & $1.1580 \mathrm{E}+11$ & $8.6358 \mathrm{E}-12$ & & & \\
\hline
\end{tabular}

WSRC-TR-99-00344

October, 1999

page 62 


\begin{tabular}{|c|c|c|c|c|c|c|c|c|c|}
\hline Isotope & $\begin{array}{c}\text { Atomic } \\
\text { Mass }\end{array}$ & Halflife & $\begin{array}{l}\text { Time } \\
\text { Units }\end{array}$ & $t_{112}, \sec$ & Curies/ gram & Grams/ Curie & $\begin{array}{c}\text { Watts/Ci } \\
\text { (Ref.3) }\end{array}$ & $\begin{array}{c}\text { Individual }^{1} \\
\text { Fission } \\
\text { Product Yield } \\
\text { (Ref.4) }\end{array}$ & $\begin{array}{c}\text { Cumulative } \\
\text { Fission Product } \\
\text { Yield (Ref.4) }\end{array}$ \\
\hline $\mathrm{Pb} 204$ & 203.9730 & $1.400 \mathrm{E}+17$ & year & $4.42 E+24$ & $1.2519 \mathrm{E}-14$ & $7.9880 E+13$ & & & \\
\hline $\mathrm{Pb} 204 \mathrm{~m}$ & 203.9730 & $6.720 \mathrm{E}+01$ & minute & $4.03 E+03$ & $1.3718 \mathrm{E}+07$ & $7.2898 \mathrm{E}-08$ & & & \\
\hline $\mathrm{Pb} 205$ & 204.9745 & $1.530 E+07$ & year & $4.83 E+14$ & $1.1399 \mathrm{E}-04$ & $8.7726 E+03$ & & & \\
\hline $\mathrm{Pb} 205 \mathrm{~m}$ & 204.9745 & $5.540 \mathrm{E}-03$ & second & $5.54 \mathrm{E}-03$ & $9.9350 \mathrm{E}+12$ & $1.0065 \mathrm{E}-13$ & & & \\
\hline $\mathrm{Pb} 207 \mathrm{~m}$ & 206.9759 & $8.050 \mathrm{E}-01$ & second & $8.05 \mathrm{E}-01$ & $6.7711 E+10$ & $1.4769 \mathrm{E}-11$ & & & \\
\hline $\mathrm{Pb} 209$ & 208.9811 & $3.253 \mathrm{E}+00$ & hour & $1.17 E+04$ & $4.6098 E+06$ & $2.1693 \mathrm{E}-07$ & 0.001170 & & \\
\hline $\mathrm{Pb} 210$ & 209.9842 & $2.230 E+01$ & year & $7.04 \mathrm{E}+08$ & $7.6343 \mathrm{E}+01$ & $1.3099 \mathrm{E}-02$ & 0.000203 & & \\
\hline $\mathrm{Pb} 211$ & 210.9887 & $3.610 E+01$ & minute & $2.17 E+03$ & $2.4686 \mathrm{E}+07$ & $4.0508 \mathrm{E}-08$ & 0.003083 & & \\
\hline $\mathrm{Pb} 212$ & 211.9919 & $1.064 \mathrm{E}+01$ & hour & $3.83 E+04$ & $1.3894 E+06$ & $7.1976 \mathrm{E}-07$ & 0.001900 & & \\
\hline $\mathrm{Pb} 213$ & 212.9965 & $1.020 E+01$ & minute & $6.12 E+02$ & $8.6547 \mathrm{E}+07$ & $1.1554 E-08$ & & & \\
\hline $\mathrm{Pb} 214$ & 213.9998 & $2.680 E+01$ & minute & $1.61 \mathrm{E}+03$ & $3.2785 \mathrm{E}+07$ & $3.0502 \mathrm{E}-08$ & & & \\
\hline Bi 187 & 186.9935 & $3.500 \mathrm{E}-02$ & second & $3.50 \mathrm{E}-02$ & $1.7238 \mathrm{E}+12$ & $5.8012 \mathrm{E}-13$ & & & \\
\hline Bi $187 \mathrm{~m}$ & 186.9935 & $8.000 \mathrm{E}-04$ & second & $8.00 \mathrm{E}-04$ & $7.5415 \mathrm{E}+13$ & $1.3260 \mathrm{E}-14$ & & & \\
\hline Bi 188 & 187.9922 & $4.400 \mathrm{E}-02$ & second & $4.40 \mathrm{E}-02$ & $1.3639 \mathrm{E}+12$ & $7.3319 \mathrm{E}-13$ & & & \\
\hline Bi 189 & 188.9895 & $6.800 \mathrm{E}-01$ & second & $6.80 \mathrm{E}-01$ & $8.7787 E+10$ & $1.1391 \mathrm{E}-11$ & & & \\
\hline Bi $189 \mathrm{~m}$ & 188.9895 & $5.000 \mathrm{E}-03$ & second & $5.00 \mathrm{E}-03$ & $1.1939 \mathrm{E}+13$ & $8.3759 \mathrm{E}-14$ & & & \\
\hline Bi 190 & 189.9885 & $5.700 E+00$ & second & $5.70 \mathrm{E}+00$ & $1.0418 \mathrm{E}+10$ & $9.5990 \mathrm{E}-11$ & & & \\
\hline Bi $190 \mathrm{~m}$ & 189.9885 & $5.900 \mathrm{E}+00$ & second & $5.90 \mathrm{E}+00$ & $1.0065 \mathrm{E}+10$ & $9.9358 \mathrm{E}-11$ & & & \\
\hline $\mathrm{Bi} 190 \mathrm{~m} 2$ & 189.9885 & $5.700 E+00$ & second & $5.70 \mathrm{E}+00$ & $1.0418 \mathrm{E}+10$ & $9.5990 \mathrm{E}-11$ & & & \\
\hline Bi 191 & 190.9860 & $1.200 E+01$ & second & $1.20 E+01$ & $4.9226 \mathrm{E}+09$ & $2.0315 E-10$ & & & \\
\hline$B i 191 \mathrm{~m}$ & 190.9860 & $1.500 \mathrm{E}-01$ & second & $1.50 \mathrm{E}-01$ & $3.9381 \mathrm{E}+11$ & $2.5393 E-12$ & & & \\
\hline Bi 192 & 191.9854 & $3.460 \mathrm{E}+01$ & second & $3.46 \mathrm{E}+01$ & $1.6984 \mathrm{E}+09$ & $5.8880 \mathrm{E}-10$ & & & \\
\hline Bi $192 m$ & 191.9854 & $4.060 E+01$ & second & $4.06 E+01$ & $1.4474 \mathrm{E}+09$ & $6.9090 \mathrm{E}-10$ & & & \\
\hline Bi 193 & 192.9831 & $6.700 \mathrm{E}+01$ & second & $6.70 \mathrm{E}+01$ & $8.7253 \mathrm{E}+08$ & $1.1461 \mathrm{E}-09$ & & & \\
\hline Bi $193 m$ & 192.9831 & $3.200 \mathrm{E}+00$ & second & $3.20 E+00$ & $1.8269 \mathrm{E}+10$ & $5.4738 \mathrm{E}-11$ & & & \\
\hline Bi 194 & 193.9827 & $9.500 \mathrm{E}+01$ & second & $9.50 E+01$ & $6.1220 \mathrm{E}+08$ & 1.6335E-09 & & & \\
\hline Bi $194 \mathrm{~m}$ & 193.9827 & $1.250 \mathrm{E}+02$ & second & $1.25 \mathrm{E}+02$ & $4.6527 \mathrm{E}+08$ & $2.1493 \mathrm{E}-09$ & & & \\
\hline Bi $194 \mathrm{~m} 2$ & 193.9827 & $1.150 E+02$ & second & $1.15 E+02$ & $5.0573 E+08$ & $1.9774 \mathrm{E}-09$ & & & \\
\hline Bi 195 & 194.9808 & $1.830 \mathrm{E}+02$ & second & $1.83 E+02$ & $3.1618 \mathrm{E}+08$ & $3.1628 \mathrm{E}-09$ & & & $\therefore$ \\
\hline $\mathrm{Bi} 195 \mathrm{~m}$ & 194.9808 & $8.700 \mathrm{E}+01$ & second & $8.70 \mathrm{E}+01$ & $6.6507 \mathrm{E}+08$ & 1.5036E-09 & & & \\
\hline Bi 196 & 195.9806 & $3.080 \mathrm{E}+02$ & second & $3.08 \mathrm{E}+02$ & $1.8690 \mathrm{E}+08$ & $5.3504 \mathrm{E}-09$ & & & \\
\hline Bi $196 \mathrm{~m}$ & 195.9806 & $6.000 \mathrm{E}-01$ & second & $6.00 \mathrm{E}-01$ & $9.5943 \mathrm{E}+10$ & $1.0423 \mathrm{E}-11$ & & & \\
\hline Bi 196m2 & 195.9806 & $2.400 E+02$ & second & $2.40 E+02$ & $2.3986 \mathrm{E}+08$ & $4.1692 \mathrm{E}-09$ & & & \\
\hline Bi 197 & 196.9789 & $9.330 E+00$ & minute & $5.60 \mathrm{E}+02$ & $1.0231 \mathrm{E}+08$ & $9.7741 \mathrm{E}-09$ & & & \\
\hline Bi $197 m$ & 196.9789 & $5.040 \mathrm{E}+00$ & minute & $3.02 E+02$ & $1.8940 \mathrm{E}+08$ & 5.2799E-09 & & & \\
\hline
\end{tabular}




\begin{tabular}{|c|c|c|c|c|c|c|c|c|c|}
\hline Isotope & $\begin{array}{l}\text { Atomic } \\
\text { Mass }\end{array}$ & Halflife & $\begin{array}{l}\text { Time } \\
\text { Units }\end{array}$ & $t_{1 / 2}, \sec$ & Curies/ gram & Grams/Curie & $\begin{array}{c}\text { Watts/Ci } \\
\text { (Ref.3) }\end{array}$ & $\begin{array}{c}\text { Individual }^{1} \\
\text { Fission } \\
\text { Product Yield } \\
\text { (Ref.4) } \\
\end{array}$ & $\begin{array}{l}\text { Cumulative } \\
\text { Fission Product } \\
\text { Yield (Ref.4) }\end{array}$ \\
\hline Bi 198 & 197.9790 & $1.030 E+01$ & minute & $6.18 E+02$ & $9.2208 \mathrm{E}+07$ & $1.0845 E-08$ & & & \\
\hline Bi $198 \mathrm{~m}$ & 197.9790 & $1.160 E+01$ & minute & $6.96 \mathrm{E}+02$ & $8.1874 E+07$ & $1.2214 \mathrm{E}-08$ & & & \\
\hline Bi 198m2 & 197.9790 & $7.700 E+00$ & second & $7.70 \mathrm{E}+00$ & $7.4006 \mathrm{E}+09$ & $1.3512 \mathrm{E}-10$ & & & \\
\hline Bi 199 & 198.9776 & $2.700 \mathrm{E}+01$ & minute & $1.62 \mathrm{E}+03$ & $3.4999 \mathrm{E}+07$ & $2.8572 E-08$ & & & \\
\hline Bi $199 m$ & 198.9776 & $2.470 \mathrm{E}+01$ & minute & $1.48 \mathrm{E}+03$ & $3.8258 \mathrm{E}+07$ & $2.6138 \mathrm{E}-08$ & & & \\
\hline $\mathrm{Bi} 200$ & 199.9781 & $3.640 E+01$ & minute & $2.18 E+03$ & $2.5831 \mathrm{E}+07$ & $3.8713 \mathrm{E}-08$ & & & \\
\hline Bi $200 \mathrm{~m}$ & 199.9781 & $3.100 E+01$ & minute & $1.86 E+03$ & $3.0331 \mathrm{E}+07$ & $3.2970 \mathrm{E}-08$ & & & \\
\hline $\mathrm{Bi} 200 \mathrm{~m} 2$ & 199.9781 & $4.000 \mathrm{E}-01$ & second & $4.00 \mathrm{E}-01$ & $1.4104 E+11$ & $7.0903 \mathrm{E}-12$ & & & \\
\hline $8 \mathrm{Bi} 201$ & 200.9770 & $1.080 E+02$ & minute & $6.48 E+03$ & $8.6627 E+06$ & $1.1544 \mathrm{E}-07$ & & & \\
\hline Bi $201 \mathrm{~m}$ & 200.9770 & $5.910 E+01$ & minute & $3.55 \mathrm{E}+03$ & $1.5830 \mathrm{E}+07$ & $6.3170 \mathrm{E}-08$ & & & \\
\hline $\mathrm{Bi} 202$ & 201.9777 & $1.720 E+00$ & hour & $6.19 \mathrm{E}+03$ & $9.0207 \mathrm{E}+06$ & $1.1086 \mathrm{E}-07$ & & & \\
\hline $\mathrm{Bi} 203$ & 202.9769 & $1.176 E+01$ & hour & $4.23 E+04$ & $1.3129 E+06$ & $7.6169 \mathrm{E}-07$ & & & \\
\hline Bi 203m & 202.9769 & $3.030 \mathrm{E}-01$ & second & $3.03 \mathrm{E}-01$ & $1.8344 \mathrm{E}+11$ & $5.4515 \mathrm{E}-12$ & & & \\
\hline Bi 204 & 203.9778 & $1.122 E+01$ & hour & $4.04 E+04$ & $1.3693 E+06$ & $7.3030 \mathrm{E}-07$ & & & \\
\hline Bi 204m & 203.9778 & $1.300 \mathrm{E}-02$ & second & $1.30 \mathrm{E}-02$ & $4.2545 E+12$ & $2.3504 \mathrm{E}-13$ & & & \\
\hline Bi 204m2 & 203.9778 & $1.070 \mathrm{E}-03$ & second & $1.07 \mathrm{E}-03$ & $5.1690 \mathrm{E}+13$ & $1.9346 \mathrm{E}-14$ & & & \\
\hline Bi 205 & 204.9774 & $1.531 E+01$ & day & $1.32 E+06$ & $4.1608 E+04$ & $2.4034 \mathrm{E}-05$ & & & \\
\hline Bi 206 & 205.9785 & $6.243 E+00$ & day & $5.39 E+05$ & $1.0154 E+05$ & 9.8481E-06 & & & \\
\hline Bi 207 & 206.9785 & $3.155 E+01$ & year & $9.96 E+08$ & $5.4744 E+01$ & 1.8267E-02 & & & \\
\hline Bi 208 & 207.9797 & $3.680 E+05$ & year & $1.16 E+13$ & $4.6708 \mathrm{E}-03$ & $2.1410 \mathrm{E}+02$ & & & \\
\hline Bi 208m & 207.9797 & $2.580 \mathrm{E}-03$ & second & $2.58 \mathrm{E}-03$ & $2.1025 \mathrm{E}+13$ & $4.7562 \mathrm{E}-14$ & & & \\
\hline$B i 210$ & 209.9841 & $5.013 E+00$ & day & $4.33 E+05$ & $1.2404 E+05$ & $8.0616 \mathrm{E}-06$ & & & \\
\hline Bi $210 \mathrm{~m}$ & 209.9841 & $3.040 E+06$ & year. & $9.59 E+13$ & $5.6002 \mathrm{E}-04$ & $1.7857 \mathrm{E}+03$ & & & \\
\hline Bi 211 & 210.9873 & $2.140 E+00$ & minute & $1.28 E+02$ & $4.1644 \mathrm{E}+08$ & $2.4013 \mathrm{E}-09$ & 0.039160 & & \\
\hline $\mathrm{Bi} 212$ & 211.9913 & $6.055 E+01$ & minute & $3.63 E+03$ & $1.4648 E+07$ & $6.8266 \mathrm{E}-08$ & 0.016490 & & \\
\hline$B i 212 m$ & 211.9913 & $2.500 \mathrm{E}+01$ & minute & $1.50 \mathrm{E}+03$ & $3.5479 E+07$ & $2.8186 \mathrm{E}-08$ & & & \\
\hline $\mathrm{Bi} 212 \mathrm{~m} 2$ & 211.9913 & $7.000 E+00$ & minute & $4.20 E+02$ & $1.2671 \mathrm{E}+08$ & 7.8921E-09 & & & \\
\hline \begin{tabular}{|l|}
$\mathrm{Bi} 213$ \\
\end{tabular} & 212.9944 & $4.559 E+01$ & minute & $2.74 E+03$ & $1.9364 E+07$ & $5.1643 \mathrm{E}-08$ & 0.003950 & & \\
\hline Bi 214 & 213.9987 & $1.990 \mathrm{E}+01$ & minute & $1.19 E+03$ & $4.4153 \mathrm{E}+07$ & $2.2648 \mathrm{E}-08$ & & & \\
\hline Bi 215 & 215.0018 & $7.600 E+00$ & minute & $4.56 \mathrm{E}+02$ & $1.1507 E+08$ & $8.6902 \mathrm{E}-09$ & & & \\
\hline Bi 216 & 216.0019 & $3.600 E+00$ & minute & $2.16 E+02$ & $2.4180 \mathrm{E}+08$ & 4.1356E-09 & & & \\
\hline Po 191 & 190.9947 & $1.550 \mathrm{E}-02$ & second & $1.55 \mathrm{E}-02$ & $3.8109 \mathrm{E}+12$ & $2.6241 \mathrm{E}-13$ & & & \\
\hline Po 192 & 191.9915 & $3.400 \mathrm{E}-02$ & second & $3.40 \mathrm{E}-02$ & $1.7283 \mathrm{E}+12$ & $5.7861 E-13$ & & & \\
\hline Po 193 & 192.9911 & 3.600E-01 & second & 3.60E-01 & $1.6238 \mathrm{E}+11$ & $6.1583 E-12$ & & & \\
\hline Po $193 \mathrm{~m}$ & 192.9911 & $2.600 \mathrm{E}-01$ & second & $2.60 \mathrm{E}-01$ & $2.2484 E+11$ & $4.4477 \mathrm{E}-12$ & & & \\
\hline
\end{tabular}

WSRC-TR-99-00344

October, 1999

page 64 
Radionuclide Specific Data

\begin{tabular}{|c|c|c|c|c|c|c|c|c|c|}
\hline Isotope & $\begin{array}{c}\text { Atomic } \\
\text { Mass }\end{array}$ & Halflife & $\begin{array}{l}\text { Time } \\
\text { Units }\end{array}$ & $t_{1 / 2}, \sec$ & Curies/ gram & Grams/ Curie & $\begin{array}{c}\text { Watts/Ci } \\
\text { (Ref.3) }\end{array}$ & $\begin{array}{c}\text { Individual }^{1} \\
\text { Fission } \\
\text { Product Yield } \\
\text { (Ref.4) } \\
\end{array}$ & $\begin{array}{c}\text { Cumulative } \\
\text { Fission Product } \\
\text { Yield (Ref.4) }\end{array}$ \\
\hline Po 194 & 193.9883 & 3.920E-01 & second & $3.92 \mathrm{E}-01$ & $1.4836 \mathrm{E}+11$ & $6.7404 \mathrm{E}-12$ & & & \\
\hline Po 195 & 194.9880 & $4.640 \mathrm{E}+00$ & second & $4.64 \mathrm{E}+00$ & $1.2470 \mathrm{E}+10$ & $8.0195 \mathrm{E}-11$ & & & \\
\hline Po $195 \mathrm{~m}$ & 194.9880 & $1.920 \mathrm{E}+00$ & second & $1.92 E+00$ & $3.0135 E+10$ & $3.3184 \mathrm{E}-11$ & & & \\
\hline Po 196 & 195.9855 & $5.800 \mathrm{E}+00$ & second & $5.80 \mathrm{E}+00$ & $9.9249 \mathrm{E}+09$ & $1.0076 E-10$ & & & \\
\hline Po 197 & 196.9856 & $5.600 \mathrm{E}+01$ & second & $5.60 \mathrm{E}+01$ & $1.0227 E+09$ & $9.7779 \mathrm{E}-10$ & & & \\
\hline Po 197m & 196.9856 & $2.600 \mathrm{E}+01$ & second & $2.60 \mathrm{E}+01$ & $2.2028 E+09$ & $4.5397 E-10$ & & & \\
\hline Po 198 & 197.9833 & $1.760 E+00$ & minute & $1.06 \mathrm{E}+02$ & $5.3961 E+08$ & $1.8532 \mathrm{E}-09$ & & & \\
\hline Po 199 & 198.9836 & $5.480 E+00$ & minute & $3.29 \mathrm{E}+02$ & $1.7244 \mathrm{E}+08$ & 5.7993E-09 & & & \\
\hline Po $199 \mathrm{~m}$ & 198.9836 & $4.130 E+00$ & minute & $2.48 \mathrm{E}+02$ & $2.2880 E+08$ & $4.3706 \mathrm{E}-09$ & & & \\
\hline Po 200 & 199.9817 & $1.150 E+01$ & minute & $6.90 \mathrm{E}+02$ & $8.1759 E+07$ & $1.2231 \mathrm{E}-08$ & & & \\
\hline Po 201 & 200.9822 & $1.530 E+01$ & minute & $9.18 \mathrm{E}+02$ & $6.1147 \mathrm{E}+07$ & $1.6354 \mathrm{E}-08$ & & & \\
\hline Po 201m & 200.9822 & $8.900 \mathrm{E}+00$ & minute & $5.34 E+02$ & $1.0512 E+08$ & $9.5131 \mathrm{E}-09$ & & & \\
\hline Po.202 & 201.9807 & $4.470 E+01$ & minute & $2.68 \mathrm{E}+03$ & $2.0826 \mathrm{E}+07$ & $4.8017 \mathrm{E}-08$ & & & \\
\hline Po 203 & 202.9814 & $3.670 E+01$ & minute & $2.20 \mathrm{E}+03$ & $2.5241 E+07$ & $3.9618 \mathrm{E}-08$ & & & \\
\hline Po 203m & 202.9814 & $1.200 E+00$ & minute & $7.20 \mathrm{E}+01$ & $7.7195 E+08$ & $1.2954 \mathrm{E}-09$ & & & \\
\hline Po 204 & 203.9803 & $3.530 \mathrm{E}+00$ & hour & $1.27 \mathrm{E}+04$ & $4.3522 \mathrm{E}+06$ & $2.2977 \mathrm{E}-07$ & & & \\
\hline Po 205 & 204.9812 & $1.660 E+00$ & hour & $5.98 \mathrm{E}+03$ & $9.2098 \mathrm{E}+06$ & $1.0858 \mathrm{E}-07$ & & & \\
\hline Po 206 & 205.9805 & $8.800 E+00$ & day & $7.60 \mathrm{E}+05$ & $7.2037 E+04$ & $1.3882 \mathrm{E}-05$ & & & \\
\hline Po 207 & 206.9816 & $5.800 E+00$ & hour & $2.09 E+04$ & $2.6104 E+06$ & $3.8308 \mathrm{E}-07$ & & & \\
\hline Po 207m & 206.9816 & $2.800 E+00$ & second & $2.80 \mathrm{E}+00$ & $1.9466 \mathrm{E}+10$ & $5.1370 \mathrm{E}-11$ & & & \\
\hline Po 208 & 207.9812 & $2.898 E+00$ & year & $9.15 E+07$ & $5.9311 \mathrm{E}+02$ & $1.6860 \mathrm{E}-03$ & & & \\
\hline Po 209 & 208.9824 & $1.020 \mathrm{E}+02$ & year & $3.22 E+09$ & $1.6771 \mathrm{E}+01$ & $5.9628 \mathrm{E}-02$ & 0.294300 & & \\
\hline Po 210 & 209.9829 & $1.384 E+02$ & day & $1.20 \mathrm{E}+07$ & $4.4938 \mathrm{E}+03$ & $2.2253 E-04$ & & & \\
\hline Po211 & 210.9866 & $5.160 \mathrm{E}-01$ & second & $5.16 \mathrm{E}-01$ & $1.0363 E+11$ & $9.6500 \mathrm{E}-12$ & & & \\
\hline Po $211 \mathrm{~m}$ & 210.9866 & $2.520 E+01$ & second & $2.52 E+01$ & $2.1219 E+09$ & $4.7128 E-10$ & & & \\
\hline Po 212 & 211.9889 & $2.990 \mathrm{E}-07$ & second & $2.99 \mathrm{E}-07$ & $1.7799 \mathrm{E}+17$ & $5.6183 \mathrm{E}-18$ & 0.052070 & & \\
\hline Po $212 m$ & 211.9889 & $4.510 E+01$ & second & $4.51 \mathrm{E}+01$ & $1.1800 \mathrm{E}+09$ & $8.4745 \mathrm{E}-10$ & & & \\
\hline Po 213 & 212.9928 & $4.200 \mathrm{E}-06$ & second & $4.20 \mathrm{E}-06$ & $1.2611 \mathrm{E}+16$ & $7.9294 \mathrm{E}-17$ & 0.049640 & & \\
\hline Po 214 & 213.9952 & $1.643 \mathrm{E}-04$ & second & 1.64E-04 & $3.2087 \mathrm{E}+14$ & $3.1165 \mathrm{E}-15$ & & & \\
\hline Po 215 & 214.9994 & $1.781 \mathrm{E}-03$ & second & $1.78 \mathrm{E}-03$ & $2.9463 E+13$ & $3.3941 E-14$ & 0.043780 & & \\
\hline Po 216 & 216.0019 & $1.450 \mathrm{E}-01$ & second & $1.45 \mathrm{E}-01$ & $3.6021 \mathrm{E}+11$ & $2.7762 \mathrm{E}-12$ & 0.040180 & & \\
\hline Po 217 & 217.0063 & $1.000 E+01$ & second & $1.00 \mathrm{E}+01$ & $5.1988 E+09$ & $1.9235 \mathrm{E}-10$ & & & \\
\hline Po 218 & 218.0090 & $3.100 E+00$ & minute & $1.86 \mathrm{E}+02$ & $2.7822 E+08$ & $3.5943 \mathrm{E}-09$ & & & \\
\hline At 194 & 193.9990 & $1.800 \mathrm{E}-01$ & second & $1.80 \mathrm{E}-01$ & $3.2308 \mathrm{E}+11$ & $3.0952 E-12$ & & & \\
\hline At 196 & 195.9957 & $3.000 \mathrm{E}-01$ & second & $3.00 \mathrm{E}-01$ & $1.9187 \mathrm{E}+11$ & $5.2118 \mathrm{E}-12$ & & & \\
\hline
\end{tabular}

WSRC-TR-99-00344

October, 1999

page 65 


\begin{tabular}{|c|c|c|c|c|c|c|c|c|c|}
\hline Isotope & $\begin{array}{l}\text { Atomic } \\
\text { Mass }\end{array}$ & Halflife & $\begin{array}{l}\text { Time } \\
\text { Units }\end{array}$ & $t_{12}, \sec$ & Curies/ gram & Grams/ Curie & $\begin{array}{c}\text { Watts/Ci } \\
\text { (Ref.3) }\end{array}$ & $\begin{array}{c}\text { Individual }^{\dagger} \\
\text { Fission } \\
\text { Product Yield } \\
\text { (Ref.4) }\end{array}$ & $\begin{array}{l}\text { Cumulative } \\
\text { Fission Product } \\
\text { Yield (Ref.4) }\end{array}$ \\
\hline At 197 & 196.9933 & $3.500 \mathrm{E}-01$ & second & $3.50 \mathrm{E}-01$ & $1.6363 \mathrm{E}+11$ & $6.1114 \mathrm{E}-12$ & & & \\
\hline At $197 \mathrm{~m}$ & 196.9933 & $3.700 E+00$ & second & $3.70 \mathrm{E}+00$ & $1.5478 E+10$ & $6.4607 \mathrm{E}-11$ & & & \\
\hline At 198 & 197.9928 & $4.200 E+00$ & second & $4.20 \mathrm{E}+00$ & $1.3567 \mathrm{E}+10$ & $7.3709 \mathrm{E}-11$ & & & \\
\hline At $198 \mathrm{~m}$ & 197.9928 & $1.000 E+00$ & second & $1.00 E+00$ & $5.6981 \mathrm{E}+10$ & $1.7550 \mathrm{E}-11$ & & & \\
\hline At 199 & 198.9906 & $7.200 E+00$ & second & $7.20 \mathrm{E}+00$ & $7.8743 E+09$ & $1.2700 \mathrm{E}-10$ & & & \\
\hline At 200 & 199.9903 & $4.300 E+01$ & second & $4.30 \mathrm{E}+01$ & $1.3119 \mathrm{E}+09$ & $7.6226 \mathrm{E}-10$ & & & \\
\hline At $200 \mathrm{~m}$ & 199.9903 & $4.700 E+01$ & second & $4.70 E+01$ & $1.2002 E+09$ & $8.3316 \mathrm{E}-10$ & & & \\
\hline At $200 \mathrm{~m} 2$ & 199.9903 & $3.500 E+00$ & second & $3.50 E+00$ & $1.6118 \mathrm{E}+10$ & $6.2044 E-11$ & & & \\
\hline At 201 & 200.9885 & $8.900 E+01$ & second & $8.90 \mathrm{E}+01$ & $6.3069 E+08$ & $1.5856 \mathrm{E}-09$ & & & \\
\hline At 202 & 201.9884 & $1.840 E+02$ & second & $1.84 \mathrm{E}+02$ & $3.0355 E+08$ & $3.2943 \mathrm{E}-09$ & & & \\
\hline At $202 \mathrm{~m}$ & 201.9884 & $1.820 E+02$ & second & $1.82 E+02$ & $3.0689 E+08$ & $3.2585 \mathrm{E}-09$ & & & \\
\hline At $202 \mathrm{~m} 2$ & 201.9884 & $4.600 \mathrm{E}-01$ & second & $4.60 \mathrm{E}-01$ & $1.2142 \mathrm{E}+11$ & $8.2358 E-12$ & & & \\
\hline At 203 & 202.9868 & $7.400 E+00$ & minute & $4.44 E+02$ & $1.2518 \mathrm{E}+08$ & $7.9887 \mathrm{E}-09$ & & & \\
\hline At 204 & 203.9873 & $9.200 E+00$ & minute & $5.52 \mathrm{E}+02$ & $1.0019 E+08$ & $9.9808 \mathrm{E}-09$ & & & \\
\hline At $204 \mathrm{~m}$ & 203.9873 & $1.080 \mathrm{E}-01$ & second & $1.08 \mathrm{E}-01$ & $5.1209 E+11$ & $1.9528 \mathrm{E}-12$ & & & \\
\hline At 205 & 204.9860 & $2.620 \mathrm{E}+01$ & minute & $1.57 E+03$ & $3.5011 E+07$ & 2.8563E-08 & & & \\
\hline At 206 & 205.9866 & $3.000 E+01$ & minute & $1.80 E+03$ & $3.0427 \mathrm{E}+07$ & $3.2865 \mathrm{E}-08$ & & & \\
\hline At 207 & 206.9858 & $1.800 \mathrm{E}+00$ & hour & $6.48 \mathrm{E}+03$ & $8.4113 E+06$ & 1.1889E-07 & & & \\
\hline At 208 & 207.9866 & $1.630 \mathrm{E}+00$ & hour & $5.87 E+03$ & $9.2438 \mathrm{E}+06$ & $1.0818 \mathrm{E}-07$ & & & \\
\hline At 209 & 208.9862 & $5.410 E+00$ & hour & $1.95 E+04$ & $2.7718 \mathrm{E}+06$ & $3.6078 \mathrm{E}-07$ & & & \\
\hline At 210 & 209.9871 & $8.100 E+00$ & hour & $2.92 E+04$ & $1.8425 \mathrm{E}+06$ & $5.4275 \mathrm{E}-07$ & & & \\
\hline At 211 & 210.9875 & $7.214 E+00$ & hour & $2.60 E+04$ & $2.0589 E+06$ & 4.8569E- 07 & & & \\
\hline At 212 & 211.9907 & $3.140 \mathrm{E}-01$ & second & $3.14 \mathrm{E}-01$ & $1.6948 \mathrm{E}+11$ & $5.9002 \mathrm{E}-12$ & & & \\
\hline At $212 m$ & 211.9907 & $1.190 \mathrm{E}-01$ & second & $1.19 \mathrm{E}-01$ & $4.4721 E+11$ & $2.2361 E-12$ & & & \\
\hline At 213 & 212.9929 & $1.250 \mathrm{E}-07$ & second & $1.25 \mathrm{E}-07$ & $4.2374 E+17$ & $2.3599 \mathrm{E}-18$ & & & \\
\hline At 214 & 213.9964 & $5.580 \mathrm{E}-07$ & second & $5.58 \mathrm{E}-07$ & $9.4479 \mathrm{E}+16$ & $1.0584 \mathrm{E}-17$ & & & \\
\hline At $214 m$ & 213.9964 & $2.650 \mathrm{E}-07$ & second & $2.65 \mathrm{E}-07$ & $1.9894 \mathrm{E}+17$ & $5.0266 \mathrm{E}-18$ & & & \\
\hline At $214 \mathrm{~m} 2$ & 213.9964 & $7.600 \mathrm{E}-07$ & second & $7.60 \mathrm{E}-07$ & $6.9368 \mathrm{E}+16$ & $1.4416 \mathrm{E}-17$ & & & \\
\hline At 215 & 214.9986 & $1.000 \mathrm{E}-04$ & second & $1.00 \mathrm{E}-04$ & $5.2474 E+14$ & $1.9057 \mathrm{E}-15$ & & & \\
\hline At 216 & 216.0024 & 3.000 E-04 & second & $3.00 \mathrm{E}-04$ & $1.7410 E+14$ & $5.7439 \mathrm{E}-15$ & & & \\
\hline At 217 & 217.0047 & $3.230 \mathrm{E}-02$ & second & $3.23 \mathrm{E}-02$ & $1.6096 \mathrm{E}+12$ & $6.2129 \mathrm{E}-13$ & 0.041890 & & \\
\hline At 218 & 218.0087 & $1.500 E+00$ & second & $1.50 \mathrm{E}+00$ & $3.4499 \mathrm{E}+10$ & $2.8986 \mathrm{E}-11$ & & & \\
\hline At 219 & 219.0113 & $5.600 E+01$ & second & $5.60 \mathrm{E}+01$ & $9.1986 E+08$ & $1.0871 \mathrm{E}-09$ & & & \\
\hline At 220 & 220.0153 & $2.240 \mathrm{E}+02$ & second & $2.24 \mathrm{E}+02$ & $2.2892 \mathrm{E}+08$ & $4.3684 \mathrm{E}-09$ & & & \\
\hline At 221 & 221.0181 & $2.300 \mathrm{E}+00$ & minute & $1.38 \mathrm{E}+02$ & $3.6989 \mathrm{E}+08$ & $2.7035 \mathrm{E}-09$ & & & \\
\hline
\end{tabular}




\begin{tabular}{|c|c|c|c|c|c|c|c|c|c|}
\hline Isotope & $\begin{array}{l}\text { Atomic } \\
\text { Mass }\end{array}$ & Halflife & $\begin{array}{l}\text { Time } \\
\text { Units }\end{array}$ & $\mathbf{t}_{1 / 2}, \sec$ & Curies/gram & Grams/ Curie & $\begin{array}{c}\text { Watts/Ci } \\
\text { (Ref.3) }\end{array}$ & $\begin{array}{c}\text { Individual }^{1} \\
\text { Fission } \\
\text { Product Yield } \\
\text { (Ref.4) }\end{array}$ & $\begin{array}{c}\text { Cumulative }^{1} \\
\text { Fission Product } \\
\text { Yield (Ref.4) }\end{array}$ \\
\hline At 222 & 222.0223 & $5.400 \mathrm{E}+01$ & second & $5.40 E+01$ & $9.4099 \mathrm{E}+08$ & $1.0627 \mathrm{E}-09$ & & & \\
\hline At 223 & 223.0253 & $5.000 E+01$ & second & $5.00 E+01$ & $1.0117 \mathrm{E}+09$ & $9.8843 \mathrm{E}-10$ & & & \\
\hline Rn 198 & 197.9988 & $5.000 \mathrm{E}-02$ & second & $5.00 \mathrm{E}-02$ & $1.1396 \mathrm{E}+12$ & $8.7752 \mathrm{E}-13$ & & & \\
\hline Rn 199 & 198.9983 & $6.200 \mathrm{E}-01$ & second & $6.20 \mathrm{E}-01$ & $9.1440 E+10$ & $1.0936 \mathrm{E}-11$ & & & \\
\hline Rn $199 \mathrm{~m}$ & 198.9983 & $3.200 \mathrm{E}-01$ & second & $3.20 \mathrm{E}-01$ & $1.7716 \mathrm{E}+11$ & $5.6445 \mathrm{E}-12$ & & & \\
\hline$R n 200$ & 199.9957 & $1.060 E+00$ & second & $1.06 \mathrm{E}+00$ & $5.3217 E+10$ & $1.8791 \mathrm{E}-11$ & & & \\
\hline Rn 201 & 200.9955 & $7.000 E+00$ & second & $7.00 \mathrm{E}+00$ & $8.0185 E+09$ & $1.2471 \mathrm{E}-10$ & & & \\
\hline Rn 201m & 200.9955 & $3.800 E+00$ & second & $3.80 \mathrm{E}+00$ & $1.4771 \mathrm{E}+10$ & $6.7701 \mathrm{E}-11$ & & & \\
\hline $\mathrm{Rn} 202$ & 201.9932 & $9.850 \mathrm{E}+00$ & second & $9.85 \mathrm{E}+00$ & $5.6703 \mathrm{E}+09$ & $1.7636 \mathrm{E}-10$ & & & \\
\hline$R \cap 203$ & 202.9933 & $4.500 E+01$ & second & $4.50 \mathrm{E}+01$ & $1.2350 \mathrm{E}+09$ & $8.0969 \mathrm{E}-10$ & & & \\
\hline Rn 203m & 202.9933 & $2.800 E+01$ & second & $2.80 \mathrm{E}+01$ & $1.9849 \mathrm{E}+09$ & $5.0381 \mathrm{E}-10$ & & & \\
\hline$R n 204$ & 203.9914 & $1.240 \mathrm{E}+00$ & minute & $7.44 \mathrm{E}+01$ & $7.4335 \mathrm{E}+08$ & 1.3453E-09 & & & \\
\hline $\operatorname{Rn} 205$ & 204.9917 & $2.800 E+00$ & minute & $1.68 \mathrm{E}+02$ & $3.2759 \mathrm{E}+08$ & $3.0526 \mathrm{E}-09$ & & & \\
\hline $\operatorname{Rn} 206$ & 205.9902 & $5.670 E+00$ & minute & $3.40 E+02$ & $1.6099 \mathrm{E}+08$ & $6.2116 \mathrm{E}-09$ & & & \\
\hline$R \cap 207$ & 206.9907 & $9.250 \mathrm{E}+00$ & minute & $5.55 E+02$ & $9.8205 \mathrm{E}+07$ & $1.0183 E-08$ & & & \\
\hline$R n 208$ & 207.9896 & $2.435 \mathrm{E}+01$ & minute & $1.46 \mathrm{E}+03$ & $3.7127 \mathrm{E}+07$ & $2.6935 \mathrm{E}-08$ & & & \\
\hline$R \cap 209$ & 208.9904 & $2.850 E+01$ & minute & $1.71 \mathrm{E}+03$ & $3.1569 E+07$ & $3.1677 \mathrm{E}-08$ & & & \\
\hline$R n 210$ & 209.9897 & $2.400 E+00$ & hour & $8.64 \mathrm{E}+03$ & $6.2182 E+06$ & $1.6082 \mathrm{E}-07$ & & & \\
\hline$R \cap 211$ & 210.9906 & $1.460 E+01$ & hour & $5.26 \mathrm{E}+04$ & $1.0173 \mathrm{E}+06$ & $9.8297 \mathrm{E}-07$ & & & \\
\hline $\operatorname{Rn} 212$ & 211.9907 & $2.390 E+01$ & minute & $1.43 E+03$ & $3.7112 E+07$ & $2.6946 E-08$ & & & \\
\hline $\mathrm{Rn} 213$ & 212.9939 & $2.500 \mathrm{E}-02$ & second & $2.50 \mathrm{E}-02$ & $2.1187 E+12$ & $4.7199 \mathrm{E}-13$ & & & \\
\hline$R n 214$ & 213.9953 & $2.700 \mathrm{E}-07$ & second & $2.70 E-07$ & $1.9526 \mathrm{E}+17$ & $5.1214 \mathrm{E}-18$ & & & \\
\hline $\operatorname{Rn} 214 m$ & 213.9953 & $7.000 \mathrm{E}-10$ & second & $7.00 \mathrm{E}-10$ & $7.5314 \mathrm{E}+19$ & $1.3278 \mathrm{E}-20$ & & & \\
\hline $\mathrm{Rn} 214 \mathrm{~m} 2$ & 213.9953 & $6.500 \mathrm{E}-09$ & second & $6.50 \mathrm{E}-09$ & $8.1107 E+18$ & $1.2329 \mathrm{E}-19$ & & & \\
\hline $\operatorname{Rn} 215$ & 214.9987 & $2.300 \mathrm{E}-06$ & second & $2.30 \mathrm{E}-06$ & $2.2815 E+16$ & $4.3832 E-17$ & & & \\
\hline $\operatorname{Rn} 216$ & 216.0003 & $4.500 \mathrm{E}-05$ & second & 4.50 E-05 & $1.1607 \mathrm{E}+15$ & $8.6157 \mathrm{E}-16$ & & & \\
\hline$R \cap 217$ & 217.0039 & $5.400 \mathrm{E}-04$ & second & $5.40 \mathrm{E}-04$ & $9.6275 E+13$ & $1.0387 \mathrm{E}-14$ & & & \\
\hline $\operatorname{Rn} 218$ & 218.0056 & $3.500 \mathrm{E}-02$ & second & $3.50 \mathrm{E}-02$ & $1.4786 E+12$ & $6.7633 \mathrm{E}-13$ & & & \\
\hline $\operatorname{Rn} 219$ & 219.0095 & $3.960 E+00$ & second & $3.96 \mathrm{E}+00$ & $1.3008 \mathrm{E}+10$ & $7.6874 \mathrm{E}-11$ & 0.040760 & & \\
\hline$R n 220$ & 220.0114 & $5.560 E+01$ & second & $5.56 \mathrm{E}+01$ & $9.2227 \mathrm{E}+08$ & $1.0843 \mathrm{E}-09$ & 0.037270 & & \\
\hline$R \cap 221$ & 221.0155 & $2.500 E+01$ & minute & $1.50 E+03$ & $3.4030 \mathrm{E}+07$ & $2.9386 \mathrm{E}-08$ & 0.032550 & & \\
\hline $\operatorname{Rn} 222$ & 222.0176 & $3.824 E+00$ & day & $3.30 \mathrm{E}+05$ & $1.5382 E+05$ & $6.5011 \mathrm{E}-06$ & & & \\
\hline$R \cap 223$ & 223.0218 & $2.320 E+01$ & minute & $1.39 \mathrm{E}+03$ & $3.6340 \mathrm{E}+07$ & $2.7518 \mathrm{E}-08$ & & & \\
\hline$R n 224$ & 224.0241 & $1.070 \mathrm{E}+02$ & minute & $6.42 E+03$ & $7.8442 \mathrm{E}+06$ & $1.2748 \mathrm{E}-07$ & & & \\
\hline$R \cap 225$ & 225.0284 & $4.500 E+00$ & minute & $2.70 \mathrm{E}+02$ & $1.8568 \mathrm{E}+08$ & $5.3855 \mathrm{E}-09$ & & & \\
\hline
\end{tabular}




\begin{tabular}{|c|c|c|c|c|c|c|c|c|c|}
\hline Isotope & $\begin{array}{c}\text { Atomic } \\
\text { Mass }\end{array}$ & Halflife & $\begin{array}{l}\text { Time } \\
\text { Units }\end{array}$ & $t_{1 / 2}, \sec$ & Curies/gram & Grams/ Curie & $\begin{array}{c}\text { Watts/Ci } \\
\text { (Ref.3) }\end{array}$ & $\begin{array}{c}\text { Individual }^{1} \\
\text { Fission } \\
\text { Product Yield } \\
\text { (Ref,4) }\end{array}$ & $\begin{array}{l}\text { Cumulative' } \\
\text { Fission Product } \\
\text { Yleld (Ref.4) }\end{array}$ \\
\hline $\operatorname{Rn} 226$ & 226.0309 & $6.000 E+00$ & minute & $3.60 \mathrm{E}+02$ & $1.3865 E+08$ & $7.2126 \mathrm{E}-09$ & & & \\
\hline$R n 227$ & 227.0354 & $2.250 E+01$ & second & $2.25 E+01$ & $2.2085 E+09$ & $4.5279 \mathrm{E}-10$ & & & \\
\hline Rn 228 & 228.0381 & $6.500 E+01$ & second & $6.50 \mathrm{E}+01$ & $7.6112 E+08$ & $1.3138 \mathrm{E}-09$ & & & \\
\hline Fr 201 & 201.0040 & $4.800 \mathrm{E}-02$ & second & $4.80 \mathrm{E}-02$ & $1.1693 \mathrm{E}+12$ & $8.5520 \mathrm{E}-13$ & & & \\
\hline Fr202 & 202.0033 & $3.400 \mathrm{E}-01$ & second & $3.40 \mathrm{E}-01$ & $1.6426 \mathrm{E}+11$ & $6.0878 \mathrm{E}-12$ & & & \\
\hline Fr 203 & 203.0010 & $5.500 \mathrm{E}-01$ & second & $5.50 \mathrm{E}-01$ & $1.0105 \mathrm{E}+11$ & $9.8966 \mathrm{E}-12$ & & & \\
\hline Fr 204 & 204.0006 & $1.700 \mathrm{E}+00$ & second & $1.70 E+00$ & $3.2531 E+10$ & $3.0740 \mathrm{E}-11$ & & & \\
\hline $\mathrm{Fr} 204 \mathrm{~m}$ & 204.0006 & $2.600 E+00$ & second & $2.60 E+00$ & $2.1270 E+10$ & $4.7014 E-11$ & & & \\
\hline Fr204m2 & 204.0006 & $1.000 E+00$ & second & $1.00 \mathrm{E}+00$ & $5.5303 E+10$ & $1.8082 \mathrm{E}-11$ & & & \\
\hline Fr 205 & 204.9987 & $3.850 E+00$ & second & $3.85 \mathrm{E}+00$ & $1.4294 \mathrm{E}+10$ & $6.9958 \mathrm{E}-11$ & & & \\
\hline Fr 206 & 205.9985 & $1.590 \mathrm{E}+01$ & second & $1.59 E+01$ & $3.4444 \mathrm{E}+09$ & $2.9033 \mathrm{E}-10$ & & & \\
\hline $\mathrm{Fr} 206 \mathrm{~m}$ & 205.9985 & $7.000 \mathrm{E}-01$ & second & $7.00 \mathrm{E}-01$ & $7.8237 E+10$ & $1.2782 \mathrm{E}-11$ & & & \\
\hline Fr 207 & 206.9969 & $1.480 E+01$ & second & $1.48 \mathrm{E}+01$ & $3.6826 \mathrm{E}+09$ & $2.7155 \mathrm{E}-10$ & & & \\
\hline Fr 208 & 207.9971 & $5.910 \mathrm{E}+01$ & second & $5.91 \mathrm{E}+01$ & $9.1777 \mathrm{E}+08$ & $1.0896 \mathrm{E}-09$ & & & \\
\hline Fr 209 & 208.9959 & $5.000 E+01$ & second & $5.00 \mathrm{E}+01$ & $1.0796 \mathrm{E}+09$ & $9.2626 \mathrm{E}-10$ & & & \\
\hline Fr 210 & 209.9964 & $3.180 E+00$ & minute & $1.91 E+02$ & $2.8157 E+08$ & $3.5515 \mathrm{E}-09$ & & & \\
\hline $\mathrm{Fr} 211$ & 210.9955 & $3.100 E+00$ & minute & $1.86 \mathrm{E}+02$ & $2.8747 \mathrm{E}+08$ & $3.4786 \mathrm{E}-09$ & & & \\
\hline $\mathrm{Fr} 212$ & 211.9962 & $2.000 E+01$ & minute & $1.20 \mathrm{E}+03$ & $4.4347 \mathrm{E}+07$ & $2.2549 \mathrm{E}-08$ & & & \\
\hline Fr 213 & 212.9962 & $3.460 E+01$ & second & $3.46 E+01$ & $1.5308 E+09$ & $6.5324 E-10$ & & & \\
\hline Fr 214 & 213.9990 & $5.000 \mathrm{E}-03$ & second & $5.00 \mathrm{E}-03$ & $1.0544 \mathrm{E}+13$ & $9.4843 E-14$ & & & \\
\hline $\mathrm{Fr} 214 \mathrm{~m}$ & 213.9990 & $3.350 \mathrm{E}-03$ & second & $3.35 \mathrm{E}-03$ & $1.5737 E+13$ & $6.3545 \mathrm{E}-14$ & & & \\
\hline Fr 215 & 215.0003 & $8.600 \mathrm{E}-08$ & second & $8.60 \mathrm{E}-08$ & $6.1015 E+17$ & $1.6389 \mathrm{E}-18$ & & & \\
\hline Fr 216 & 216.0032 & $7.000 \mathrm{E}-07$ & second & $7.00 \mathrm{E}-07$ & $7.4614 E+16$ & $1.3402 \mathrm{E}-17$ & & & \\
\hline Fr 217 & 217.0046 & $2.200 \mathrm{E}-05$ & second & $2.20 \mathrm{E}-05$ & $2.3631 E+15$ & $4.2317 \mathrm{E}-16$ & & & \\
\hline Fr218 & 218.0076 & $1.000 \mathrm{E}-03$ & second & 1.00E-03 & $5.1749 \mathrm{E}+13$ & $1.9324 \mathrm{E}-14$ & & & \\
\hline $\mathrm{Fr} 218 \mathrm{~m}$ & 218.0076 & $2.200 \mathrm{E}-02$ & second & $2.20 \mathrm{E}-02$ & $2.3522 \mathrm{E}+12$ & $4.2513 \mathrm{E}-13$ & & & \\
\hline Fr 219 & 219.0092 & $2.000 \mathrm{E}-02$ & second & $2.00 \mathrm{E}-02$ & $2.5756 \mathrm{E}+12$ & $3.8825 \mathrm{E}-13$ & & & \\
\hline Fr 220 & 220.0123 & $2.740 \mathrm{E}+01$ & second & $2.74 \mathrm{E}+01$ & $1.8715 E+09$ & $5.3434 \mathrm{E}-10$ & & & \\
\hline Fr 221 & 221.0142 & $4.900 E+00$ & minute & $2.94 E+02$ & $1.7362 E+08$ & $5.7596 \mathrm{E}-09$ & 0.037890 & & \\
\hline Fr 222 & 222.0175 & $1.420 \mathrm{E}+01$ & minute & $8.52 E+02$ & $5.9642 \mathrm{E}+07$ & 1.6767E-08 & & & \\
\hline Fr 223 & 223.0197 & $2.180 E+01$ & minute & $1.31 \mathrm{E}+03$ & $3.8675 \mathrm{E}+07$ & $2.5857 \mathrm{E}-08$ & 0.002850 & & \\
\hline Fr 224 & 224.0232 & $3.300 \mathrm{E}+00$ & minute & $1.98 \mathrm{E}+02$ & $2.5434 \mathrm{E}+08$ & $3.9317 \mathrm{E}-09$ & & & \\
\hline Fr 225 & 225.0256 & $4.000 E+00$ & minute & $2.40 \mathrm{E}+02$ & $2.0890 E+08$ & $4.7870 \mathrm{E}-09$ & & & \\
\hline Fr 226 & 226.0293 & $4.800 E+01$ & second & $4.80 E+01$ & $1.0398 E+09$ & $9.6168 \mathrm{E}-10$ & & & \\
\hline Fr 227 & 227.0318 & $2.470 E+00$ & minute & $1.48 \mathrm{E}+02$ & $3.3531 \mathrm{E}+08$ & $2.9823 \mathrm{E}-09$ & & & \\
\hline
\end{tabular}




\begin{tabular}{|c|c|c|c|c|c|c|c|c|c|}
\hline Isotope & $\begin{array}{l}\text { Atomic } \\
\text { Mass }\end{array}$ & Halflife & $\begin{array}{l}\text { Time } \\
\text { Units }\end{array}$ & $t_{1 / 2}, \sec$ & Curies/ gram & Grams/ Curie & $\begin{array}{c}\text { Watts/Cl } \\
\text { (Ref.3) }\end{array}$ & $\begin{array}{c}\text { Individual }^{1} \\
\text { Fission } \\
\text { Product Yield } \\
\text { (Ref.4) }^{\text {(R) }}\end{array}$ & $\begin{array}{l}\text { Cumulative }^{1} \\
\text { Fission Product } \\
\text { Yield (Ref.4) }\end{array}$ \\
\hline Fr 228 & 228.0357 & $3.900 E+01$ & second & $3.90 E+01$ & $1.2686 \mathrm{E}+09$ & $7.8830 \mathrm{E}-10$ & & & \\
\hline Fr 229 & 229.0384 & $5.000 \mathrm{E}+01$ & second & $5.00 E+01$ & $9.8514 \mathrm{E}+08$ & $1.0151 \mathrm{E}-09$ & & & \\
\hline Fr 230 & 230.0425 & $1.910 E+01$ & second & $1.91 E+01$ & $2.5676 \mathrm{E}+09$ & $3.8946 E-10$ & & & \\
\hline Fr 231 & 231.0454 & $1.750 E+01$ & second & $1.75 E+01$ & $2.7902 E+09$ & $3.5839 \mathrm{E}-10$ & & & \\
\hline Fr 232 & 232.0497 & $5.000 \mathrm{E}+00$ & second & $5.00 \mathrm{E}+00$ & $9.7236 \mathrm{E}+09$ & $1.0284 \mathrm{E}-10$ & & & \\
\hline Ra 205 & 205.0062 & $2.200 E-01$ & second & $2.20 \mathrm{E}-01$ & $2.5014 E+11$ & $3.9977 \mathrm{E}-12$ & & & \\
\hline $\operatorname{Ra} 206$ & 206.0038 & $2.400 \mathrm{E}-01$ & second & $2.40 \mathrm{E}-01$ & $2.2819 \mathrm{E}+11$ & $4.3824 E-12$ & & & \\
\hline $\operatorname{Ra} 207$ & 207.0037 & $1.300 \mathrm{E}+00$ & second & $1.30 \mathrm{E}+00$ & $4.1923 E+10$ & $2.3853 \mathrm{E}-11$ & & & \\
\hline Ra 207m & 207.0037 & $5.500 E-02$ & second & $5.50 \mathrm{E}-02$ & $9.9091 \mathrm{E}+11$ & $1.0092 \mathrm{E}-12$ & & & \\
\hline Ra 208 & 208.0018 & $1.300 \mathrm{E}+00$ & second & $1.30 E+00$ & $4.1722 E+10$ & $2.3968 \mathrm{E}-11$ & & & \\
\hline $\operatorname{Ra} 209$ & 209.0019 & $4.600 E+00$ & second & $4.60 E+00$ & $1.1735 E+10$ & $8.5218 \mathrm{E}-11$ & & & \\
\hline Ra 210 & 210.0004 & $3.700 E+00$ & second & $3.70 E+00$ & $1.4520 \mathrm{E}+10$ & $6.8872 E-11$ & & & \\
\hline Ra 211 & 211.0009 & $1.300 \mathrm{E}+01$ & second & $1.30 \mathrm{E}+01$ & $4.1129 \mathrm{E}+09$ & $2.4314 \mathrm{E}-10$ & & & \\
\hline $\operatorname{Ra} 212$ & 211.9998 & $1.300 E+01$ & second & $1.30 \mathrm{E}+01$ & $4.0935 \mathrm{E}+09$ & $2.4429 \mathrm{E}-10$ & & & \\
\hline $\operatorname{Ra} 213$ & 213.0003 & $2.740 \mathrm{E}+00$ & minute & $1.64 E+02$ & $3.2218 E+08$ & $3.1039 \mathrm{E}-09$ & & & \\
\hline $\operatorname{Ra} 213 \mathrm{~m}$ & 213.0003 & $2.100 \mathrm{E}-03$ & second & $2.10 \mathrm{E}-03$ & $2.5222 \mathrm{E}+13$ & $3.9648 \mathrm{E}-14$ & & & 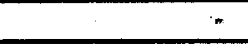 \\
\hline Ra 214 & 214.0001 & $2.460 E+00$ & second & $2.46 E+00$ & $2.1430 E+10$ & $4.6663 \mathrm{E}-11$ & & & \\
\hline Ra 215 & 215.0027 & $1.590 \mathrm{E}-03$ & second & $1.59 \mathrm{E}-03$ & $3.3002 E+13$ & $3.0302 \mathrm{E}-14$ & & & \\
\hline $\operatorname{Ra} 216$ & 216.0035 & $1.820 \mathrm{E}-07$ & second & $1.82 E-07$ & $2.8698 \mathrm{E}+17$ & $3.4846 \mathrm{E}-18$ & & & \\
\hline $\operatorname{Ra} 217$ & 217.0063 & $1.600 \mathrm{E}-06$ & second & 1.60E-06 & $3.2493 E+16$ & $3.0776 \mathrm{E}-17$ & & & \\
\hline $\operatorname{Ra218}$ & 218.0071 & $2.560 \mathrm{E}-05$ & second & $2.56 \mathrm{E}-05$ & $2.0215 E+15$ & $4.9469 \mathrm{E}-16$ & & & \\
\hline $\operatorname{Ra} 219$ & 219.0101 & $1.000 \mathrm{E}-02$ & second & $1.00 \mathrm{E}-02$ & $5.1512 E+12$ & $1.9413 \mathrm{E}-13$ & & & \\
\hline $\operatorname{Ra} 220$ & 220.0110 & $1.800 \mathrm{E}-02$ & second & $1.80 \mathrm{E}-02$ & $2.8488 E+12$ & $3.5103 \mathrm{E}-13$ & & & \\
\hline $\operatorname{Ra} 221$ & 221.0139 & $2.800 E+01$ & second & $2.80 E+01$ & $1.8231 \mathrm{E}+09$ & $5.4853 \mathrm{E}-10$ & & & \\
\hline$\overline{\operatorname{Ra} 222}$ & 222.0154 & $3.800 E+01$ & second & $3.80 E+01$ & $1.3372 E+09$ & $7.4781 \mathrm{E}-10$ & & & \\
\hline $\operatorname{Ra223}$ & 223.0185 & $1.144 \mathrm{E}+01$ & day & $9.88 E+05$ & $5.1202 E+04$ & $1.9531 E-05$ & 0.035000 & & \\
\hline Ra 224 & 224.0202 & $3.660 \mathrm{E}+00$ & day & $3.16 \mathrm{E}+05$ & $1.5926 \mathrm{E}+05$ & $6.2792 \mathrm{E}-06$ & 0.033720 & & \\
\hline $\operatorname{Ra225}$ & 225.0236 & $1.490 \mathrm{E}+01$ & day & $1.29 E+06$ & $3.8945 \mathrm{E}+04$ & $2.5677 \mathrm{E}-05$ & 0.000708 & & \\
\hline $\operatorname{Ra} 226$ & 226.0254 & $1.600 E+03$ & year & $5.05 E+10$ & $9.8852 \mathrm{E}-01$ & $1.0116 \mathrm{E}+00$ & 0.028360 & & \\
\hline $\operatorname{Ra} 227$ & 227.0292 & $4.220 E+01$ & minute & $2.53 E+03$ & $1.9626 \mathrm{E}+07$ & $5.0953 \mathrm{E}-08$ & & & \\
\hline Ra 228 & 228.0311 & $5.750 E+00$ & year & $1.81 E+08$ & $2.7265 \mathrm{E}+02$ & $3.6678 \mathrm{E}-03$ & 0.000069 & & \\
\hline Ra 229 & 229.0348 & $4.000 E+00$ & minute & $2.40 \mathrm{E}+02$ & $2.0524 E+08$ & $4.8723 \mathrm{E}-09$ & & & \\
\hline $\operatorname{Ra} 230$ & 230.0371 & $9.300 E+01$ & minute & $5.58 \mathrm{E}+03$ & $8.7891 \mathrm{E}+06$ & $1.1378 \mathrm{E}-07$ & & & \\
\hline Ra 231 & 231.0412 & $1.030 \mathrm{E}+02$ & second & $1.03 E+02$ & $4.7408 \mathrm{E}+08$ & $2.1094 E-09$ & & & \\
\hline $\operatorname{Ra} 232$ & 232.0437 & $2.500 \mathrm{E}+02$ & second & $2.50 \mathrm{E}+02$ & $1.9448 \mathrm{E}+08$ & $5.1420 \mathrm{E}-09$ & & & \\
\hline
\end{tabular}

\author{
WSRC-TR-99-00344 \\ October, 1999 \\ page 69
}




\begin{tabular}{|c|c|c|c|c|c|c|c|c|c|}
\hline Isotope & $\begin{array}{l}\text { Atomic } \\
\text { Mass }\end{array}$ & Halflife & $\begin{array}{l}\text { Time } \\
\text { Units }\end{array}$ & $t_{12}, \mathrm{sec}$ & Curies/gram & Grams/Curie & $\begin{array}{c}\text { Watts/Ci } \\
\text { (Ref.3) }\end{array}$ & \begin{tabular}{|c|} 
Individual $^{1}$ \\
Fission \\
Product Yield \\
(Ref.4)
\end{tabular} & $\begin{array}{c}\text { Cumulative }^{4} \\
\text { Fission Product } \\
\text { Yield (Ref.4) }\end{array}$ \\
\hline Fr 228 & 228.0357 & $3.900 E+01$ & second & $3.90 \mathrm{E}+01$ & $1.2686 \mathrm{E}+09$ & $7.8830 \mathrm{E}-10$ & & & \\
\hline Fr 229 & 229.0384 & $5.000 E+01$ & second & $5.00 E+01$ & $9.8514 E+08$ & $1.0151 \mathrm{E}-09$ & & & \\
\hline Fr230 & 230.0425 & $1.910 E+01$ & second & $1.91 E+01$ & $2.5676 E+09$ & $3.8946 \mathrm{E}-10$ & & & \\
\hline Fr 231 & 231.0454 & $1.750 E+01$ & second & $1.75 E+01$ & $2.7902 E+09$ & $3.5839 \mathrm{E}-10$ & & & \\
\hline Fr 232 & 232.0497 & $5.000 E+00$ & second & $5.00 \mathrm{E}+00$ & $9.7236 E+09$ & $1.0284 E-10$ & & & \\
\hline $\operatorname{Ra} 205$ & 205.0062 & $2.200 \mathrm{E}-01$ & second & $2.20 \mathrm{E}-01$ & $2.5014 E+11$ & $3.9977 \mathrm{E}-12$ & & & \\
\hline $\operatorname{Ra} 206$ & 206.0038 & $2.400 \mathrm{E}-01$ & second & $2.40 \mathrm{E}-01$ & $2.2819 E+11$ & $4.3824 \mathrm{E}-12$ & & & \\
\hline $\operatorname{Ra} 207$ & 207.0037 & $1.300 E+00$ & second & $1.30 E+00$ & $4.1923 E+10$ & $2.3853 \mathrm{E}-11$ & & & \\
\hline $\operatorname{Ra} 207 \mathrm{~m}$ & 207.0037 & $5.500 \mathrm{E}-02$ & second & $5.50 \mathrm{E}-02$ & $9.9091 \mathrm{E}+11$ & $1.0092 \mathrm{E}-12$ & & & \\
\hline $\operatorname{Ra} 208$ & 208.0018 & $1.300 E+00$ & second & $1.30 E+00$ & $4.1722 E+10$ & $2.3968 \mathrm{E}-11$ & & & \\
\hline$\overline{R a 209}$ & 209.0019 & $4.600 E+00$ & second & $4.60 E+00$ & $1.1735 E+10$ & $8.5218 E-11$ & & & \\
\hline $\operatorname{Ra} 210$ & 210.0004 & $3.700 E+00$ & second & $3.70 E+00$ & $1.4520 E+10$ & $6.8872 E-11$ & & & \\
\hline $\operatorname{Ra} 211$ & 211.0009 & $1.300 \mathrm{E}+01$ & second & $1.30 \mathrm{E}+01$ & $4.1129 E+09$ & $2.4314 \mathrm{E}-10$ & & & \\
\hline Ra 212 & 211.9998 & $1.300 E+01$ & second & $1.30 \mathrm{E}+01$ & $4.0935 E+09$ & $2.4429 \mathrm{E}-10$ & & & \\
\hline $\operatorname{Ra} 213$ & 213.0003 & $2.740 E+00$ & minute & $1.64 E+02$ & $3.2218 \mathrm{E}+08$ & $3.1039 \mathrm{E}-09$ & & & \\
\hline $\operatorname{Ra} 213 m$ & 213.0003 & $2.100 \mathrm{E}-03$ & second & $2.10 \mathrm{E}-03$ & $2.5222 E+13$ & $3.9648 \mathrm{E}-14$ & & & \\
\hline $\operatorname{Ra214}$ & 214.0001 & $2.460 \mathrm{E}+00$ & second & $2.46 \mathrm{E}+00$ & $2.1430 E+10$ & $4.6663 \mathrm{E}-11$ & & & \\
\hline$\overline{\operatorname{Ra} 215}$ & 215.0027 & $1.590 \mathrm{E}-03$ & second & $1.59 \mathrm{E}-03$ & $3.3002 \mathrm{E}+13$ & $3.0302 \mathrm{E}-14$ & & & \\
\hline $\operatorname{Ra} 216$ & 216.0035 & $1.820 \mathrm{E}-07$ & second & $1.82 \mathrm{E}-07$ & $2.8698 \mathrm{E}+17$ & $3.4846 \mathrm{E}-18$ & & & \\
\hline $\operatorname{Ra} 217$ & 217.0063 & $1.600 \mathrm{E}-06$ & second & $1.60 \mathrm{E}-06$ & $3.2493 E+16$ & 3.0776 E-17 & & & \\
\hline $\operatorname{Ra218}$ & 218.0071 & $2.560 \mathrm{E}-05$ & second & $2.56 E-05$ & $2.0215 E+15$ & $4.9469 \mathrm{E}-16$ & & & \\
\hline $\operatorname{Ra} 219$ & 219.0101 & $1.000 \mathrm{E}-02$ & second & $1.00 \mathrm{E}-02$ & $5.1512 E+12$ & $1.9413 \mathrm{E}-13$ & & & \\
\hline $\operatorname{Ra} 220$ & 220.0110 & $1.800 \mathrm{E}-02$ & second & $1.80 \mathrm{E}-02$ & $2.8488 E+12$ & $3.5103 \mathrm{E}-13$ & & & \\
\hline$\overline{\operatorname{Ra} 221}$ & 221.0139 & $2.800 E+01$ & second & $2.80 E+01$ & $1.8231 E+09$ & $5.4853 \mathrm{E}-10$ & & & \\
\hline Ra 222 & 222.0154 & $3.800 \mathrm{E}+01$ & second & $3.80 E+01$ & $1.3372 E+09$ & $7.4781 \mathrm{E}-10$ & & & \\
\hline $\operatorname{Ra} 223$ & 223.0185 & $1.144 E+01$ & day & $9.88 E+05$ & $5.1202 E+04$ & $1.9531 \mathrm{E}-05$ & 0.035000 & & \\
\hline$\longdiv { \operatorname { R a } 2 2 4 }$ & 224.0202 & $3.660 E+00$ & day & $3.16 E+05$ & $1.5926 \mathrm{E}+05$ & $6.2792 \mathrm{E}-06$ & 0.033720 & & \\
\hline $\operatorname{Ra} 225$ & 225.0236 & $1,490 \mathrm{E}+01$ & day & $1.29 E+06$ & $3.8945 E+04$ & $2.5677 \mathrm{E}-05$ & 0.000708 & & \\
\hline $\operatorname{Ra} 226$ & 226.0254 & $1.600 \mathrm{E}+03$ & year & $5.05 E+10$ & $9.8852 \mathrm{E}-01$ & $1.0116 \mathrm{E}+00$ & 0.028360 & & \\
\hline Ra 227 & 227.0292 & $4.220 E+01$ & minute & $2.53 E+03$ & $1.9626 \mathrm{E}+07$ & $5.0953 \mathrm{E}-08$ & & & \\
\hline Ra 228 & 228.0311 & $5.750 E+00$ & year & $1.81 E+08$ & $2.7265 \mathrm{E}+02$ & $3.6678 \mathrm{E}-03$ & 0.000069 & & \\
\hline Ra 229 & 229.0348 & $4.000 E+00$ & minute & $2.40 \mathrm{E}+02$ & $2.0524 E+08$ & 4.8723E-09 & & & \\
\hline $\begin{array}{l}\operatorname{Ra} 230 \\
\end{array}$ & 230.0371 & $9.300 E+01$ & minute & $5.58 E+03$ & $8.7891 \mathrm{E}+06$ & $1.1378 \mathrm{E}-07$ & & & \\
\hline $\operatorname{Ra} 231$ & 231.0412 & $1.030 \mathrm{E}+02$ & second & $1.03 E+02$ & $4.7408 \mathrm{E}+08$ & $2.1094 \mathrm{E}-09$ & & & \\
\hline Ra 232 & 232.0437 & $2.500 E+02$ & second & $2.50 E+02$ & $1.9448 \mathrm{E}+08$ & $5.1420 \mathrm{E}-09$ & & & \\
\hline
\end{tabular}

WSRC-TR-99-00344

October, 1999

page 69 
Radionuclide Specific Data

\begin{tabular}{|c|c|c|c|c|c|c|c|c|c|}
\hline Isotope & $\begin{array}{c}\text { Atomic } \\
\text { Mass }\end{array}$ & Halflife & $\begin{array}{l}\text { Time } \\
\text { Units }\end{array}$ & $t_{1 / 2}$, sec & Curies/gram & Grams/ Curie & $\begin{array}{c}\text { Watts/Ci } \\
\text { (Ref.3) }\end{array}$ & $\begin{array}{c}\text { Individual }^{1} \\
\text { Fission } \\
\text { Product Yield } \\
\text { (Ref.4) }\end{array}$ & $\begin{array}{c}\text { Cumulative }^{1} \\
\text { Fission Product } \\
\text { Yield (Ref.4) } \\
\end{array}$ \\
\hline $\operatorname{Ra} 233$ & 233.0480 & $3.000 E+01$ & second & $3.00 \mathrm{E}+01$ & $1.6137 \mathrm{E}+09$ & $6.1971 \mathrm{E}-10$ & & & \\
\hline $\operatorname{Ra} 234$ & 234.0505 & $3.000 E+01$ & second & $3.00 E+01$ & $1.6067 \mathrm{E}+09$ & $6.2238 \mathrm{E}-10$ & & & \\
\hline$A C 208$ & 208.0018 & $9.500 \mathrm{E}-02$ & second & $9.50 \mathrm{E}-02$ & $5.7093 \mathrm{E}+11$ & $1.7515 \mathrm{E}-12$ & & & \\
\hline Ac 208m & 208.0018 & $2.500 \mathrm{E}-02$ & second & $2.50 \mathrm{E}-02$ & $2.1695 E+12$ & $4.6093 \mathrm{E}-13$ & & & \\
\hline Ac 209 & 209.0096 & $1.000 \mathrm{E}-01$ & second & $1.00 \mathrm{E}-01$ & $5.3977 \mathrm{E}+11$ & $1.8526 \mathrm{E}-12$ & & & \\
\hline Ac 210 & 210.0093 & $3.500 \mathrm{E}-01$ & second & $3.50 \mathrm{E}-01$ & $1.5349 \mathrm{E}+11$ & $6.5152 E-12$ & & & \\
\hline Ac 211 & 211.0076 & $2.500 \mathrm{E}-01$ & second & $2.50 \mathrm{E}-01$ & $2.1386 \mathrm{E}+11$ & $4.6759 \mathrm{E}-12$ & & & \\
\hline Ac 212 & 212.0078 & $9.300 \mathrm{E}-01$ & second & $9.30 \mathrm{E}-01$ & $5.7219 E+10$ & $1.7477 \mathrm{E}-11$ & & & \\
\hline Ac 213 & 213.0066 & $8.000 E-01$ & second & $8.00 E-01$ & $6.6205 \mathrm{E}+10$ & $1.5105 \mathrm{E}-11$ & & & \\
\hline Ac 214 & 214.0069 & $8.200 E+00$ & second & $8.20 \mathrm{E}+00$ & $6.4289 \mathrm{E}+09$ & $1.5555 \mathrm{E}-10$ & & & \\
\hline Ac 215 & 215.0065 & $1.700 \mathrm{E}-01$ & second & $1.70 \mathrm{E}-01$ & $3.0866 \mathrm{E}+11$ & $3.2398 \mathrm{E}-12$ & & & \\
\hline Ac 216 & 216.0087 & $3.300 E-04$ & second & $3.30 \mathrm{E}-04$ & $1.5827 \mathrm{E}+14$ & $6.3184 \mathrm{E}-15$ & & & \\
\hline Ac $216 \mathrm{~m}$ & 216.0087 & $3.300 \mathrm{E}-04$ & second & $3.30 \mathrm{E}-04$ & $1.5827 E+14$ & $6.3184 \mathrm{E}-15$ & & & \\
\hline Ac 217 & 217.0093 & $6.900 \mathrm{E}-08$ & second & $6.90 \mathrm{E}-08$ & $7.5344 E+17$ & $1.3272 E-18$ & & & \\
\hline Ac $217 m$ & 217.0093 & $7.400 \mathrm{E}-07$ & second & $7.40 \mathrm{E}-07$ & $7.0253 E+16$ & $1.4234 \mathrm{E}-17$ & & & \\
\hline Ac 218 & 218.0016 & $1.080 \mathrm{E}-06$ & second & $1.08 \mathrm{E}-06$ & $4.7917 E+16$ & $2.0869 \mathrm{E}-17$ & & & \\
\hline Ac 219 & 219.0124 & $1.180 \mathrm{E}-05$ & second & $1.18 \mathrm{E}-05$ & $4.3654 E+15$ & $2.2907 \mathrm{E}-16$ & & & \\
\hline Ac 220 & 220.0156 & $2.610 \mathrm{E}-02$ & second & $2.61 \mathrm{E}-02$ & $1.9646 E+12$ & $5.0900 \mathrm{E}-13$ & & & \\
\hline Ac 221 & 221.0156 & $5.200 \mathrm{E}-02$ & second & $5.20 \mathrm{E}-02$ & $9.8164 \mathrm{E}+11$ & $1.0187 \mathrm{E}-12$ & & & \\
\hline$A c 222$ & 222.0178 & $5.000 E+00$ & second & $5.00 \mathrm{E}+00$ & $1.0163 E+10$ & $9.8397 \mathrm{E}-11$ & & & \\
\hline Ac $222 m$ & 222.0178 & $6.300 E+01$ & second & $6.30 \mathrm{E}+01$ & $8.0658 \mathrm{E}+08$ & $1.2398 \mathrm{E}-09$ & & & \\
\hline Ac 223 & 223.0191 & $2.100 E+00$ & minute & $1.26 \mathrm{E}+02$ & $4.0148 E+08$ & $2.4908 \mathrm{E}-09$ & & & \\
\hline Ac 224 & 224.0217 & $2.900 E+00$ & hour. & $1.04 \mathrm{E}+04$ & $4.8238 E+06$ & $2.0731 \mathrm{E}-07$ & & & \\
\hline Ac 225 & 225.0232 & $1.000 E+01$ & day & $8.64 \mathrm{E}+05$ & $5.8028 \mathrm{E}+04$ & $1.7233 \mathrm{E}-05$ & 0.034340 & & \\
\hline Ac 226 & 226.0261 & $2.937 E+01$ & hour & $1.06 E+05$ & $4.7208 \mathrm{E}+05$ & $2.1183 \mathrm{E}-06$ & & & \\
\hline Ac 227 & 227.0277 & $2.177 E+01$ & year & $6.87 \mathrm{E}+08$ & $7.2321 E+01$ & 1.3827E-02 & 0.000474 & & \\
\hline Ac 228 & 228.0310 & $6.150 E+00$ & hour & $2.21 E+04$ & $2.2346 E+06$ & $4.4750 \mathrm{E}-07$ & 0.008038 & & \\
\hline Ac 229 & 229.0329 & $6.270 \mathrm{E}+01$ & minute & $3.76 \mathrm{E}+03$ & $1.3094 \mathrm{E}+07$ & $7.6373 \mathrm{E}-08$ & & & \\
\hline Ac 230 & 230.0360 & $1.220 E+02$ & second & $1.22 \mathrm{E}+02$ & $4.0200 \mathrm{E}+08$ & $2.4876 E-09$ & & & \\
\hline Ac 231 & 231.0386 & $7.500 E+00$ & minute & $4.50 \mathrm{E}+02$ & $1.0851 E+08$ & $9.2155 E-09$ & & & \\
\hline Ac 232 & 232.0420 & $1.190 \mathrm{E}+02$ & second & $1.19 \mathrm{E}+02$ & $4.0857 E+08$ & $2.4476 \mathrm{E}-09$ & & & \\
\hline Ac 233 & 233.0416 & $1.450 E+02$ & second & $1.45 \mathrm{E}+02$ & $3.3387 E+08$ & $2.9952 \mathrm{E}-09$ & & & \\
\hline Ac 234 & 234.0484 & $4.400 E+01$ & second & $4.40 E+01$ & $1.0955 \mathrm{E}+09$ & $9.1281 \mathrm{E}-10$ & & & \\
\hline Th 212 & 212.0129 & $3.000 \mathrm{E}-02$ & second & $3.00 \mathrm{E}-02$ & $1.7738 \mathrm{E}+12$ & $5.6378 \mathrm{E}-13$ & & & \\
\hline Th 213 & 213.0130 & $1.400 \mathrm{E}-01$ & second & $1.40 \mathrm{E}-01$ & $3.7831 \mathrm{E}+11$ & $2.6434 \mathrm{E}-12$ & & & \\
\hline
\end{tabular}

WSRC-TR-99-00344

October, 1999

page 70 


\begin{tabular}{|c|c|c|c|c|c|c|c|c|c|}
\hline Isotope & $\begin{array}{l}\text { Atomic } \\
\text { Mass }\end{array}$ & Halflife & $\begin{array}{l}\text { Time } \\
\text { Units }\end{array}$ & $t_{1 / 2}, \sec$ & Curies/ gram & Grams/ Curie & $\begin{array}{c}\text { Watts/Ci } \\
\text { (Ref.3) }\end{array}$ & $\begin{array}{c}\text { Individual }^{4} \\
\text { Fission } \\
\text { Product Yield } \\
\text { (Ref.4) } \\
\end{array}$ & $\begin{array}{c}\text { Cumulative } \\
\text { Fission Product } \\
\text { Yield (Ref.4) } \\
\end{array}$ \\
\hline Th 214 & 214.0115 & $1.000 \mathrm{E}-01$ & second & $1.00 \mathrm{E}-01$ & $5.2716 E+11$ & $1.8970 \mathrm{E}-12$ & & & \\
\hline Th 215 & 215.0117 & $1.200 \mathrm{E}+00$ & second & $1.20 E+00$ & $4.3725 E+10$ & $2.2870 \mathrm{E}-11$ & & & \\
\hline Th 216 & 216.0111 & $2.800 E-02$ & second & $2.80 E-02$ & $1.8653 E+12$ & $5.3611 E-13$ & & & \\
\hline Th $216 \mathrm{~m}$ & 216.0111 & $1.800 \mathrm{E}-04$ & second & $1.80 \mathrm{E}-04$ & $2.9015 E+14$ & $3.4464 \mathrm{E}-15$ & & & \\
\hline Th 217 & 217.0131 & $2.520 \mathrm{E}-04$ & second & $2.52 \mathrm{E}-04$ & $2.0630 E+14$ & $4.8474 E-15$ & & & \\
\hline Th 218 & 218.0133 & $1.090 \mathrm{E}-07$ & second & $1.09 \mathrm{E}-07$ & $4.7475 \mathrm{E}+17$ & $2.1064 \mathrm{E}-18$ & & & \\
\hline Th 219 & 219.0155 & $1.050 \mathrm{E}-06$ & second & 1.05E-06 & $4.9058 E+16$ & $2.0384 \mathrm{E}-17$ & & & \\
\hline $\operatorname{Th} 220$ & 220.0157 & $9.700 \mathrm{E}-06$ & second & $9.70 \mathrm{E}-06$ & $5.2863 E+15$ & $1.8917 E-16$ & & & \\
\hline Th 221 & 221.0182 & $1.680 \mathrm{E}-03$ & second & $1.68 \mathrm{E}-03$ & $3.0384 E+13$ & $3.2912 \mathrm{E}-14$ & & & \\
\hline Th 222 & 222.0185 & $2.800 \mathrm{E}-03$ & second & $2.80 \mathrm{E}-03$ & $1.8148 \mathrm{E}+13$ & $5.5102 E-14$ & & & \\
\hline $\operatorname{Th} 223$ & 223.0208 & $6.000 \mathrm{E}-01$ & second & $6.00 \mathrm{E}-01$ & $8.4310 E+10$ & $1.1861 \mathrm{E}-11$ & & & \\
\hline Th 224 & 224.0215 & $1.050 E+00$ & second & $1.05 E+00$ & $4.7962 E+10$ & $2.0850 \mathrm{E}-11$ & & & \\
\hline Th 225 & 225.0239 & $8.720 E+00$ & minute & $5.23 E+02$ & $9.5825 \mathrm{E}+07$ & $1.0436 \mathrm{E}-08$ & & & \\
\hline Th 226 & 226.0249 & $3.090 E+01$ & minute & $1.85 E+03$ & $2.6922 \mathrm{E}+07$ & $3.7144 \mathrm{E}-08$ & & & \\
\hline Th 227 & 227.0277 & $1.872 E+01$ & day & $1.62 \mathrm{E}+06$ & $3.0724 E+04$ & $3.2548 \mathrm{E}-05$ & 0.035970 & & \\
\hline Th 228 & 228.0287 & $1.913 E+00$ & year & $6.04 E+07$ & $8.1947 E+02$ & $1.2203 \mathrm{E}-03$ & 0.032140 & & \\
\hline Th 229 & 229.0318 & $7.340 E+03$ & year & $2.32 E+11$ & $2.1265 \mathrm{E}-01$ & $4.7025 E+00$ & 0.029020 & & \\
\hline Th 230 & 230.0331 & $7.538 E+04$ & year & $2.38 E+12$ & $2.0616 \mathrm{E}-02$ & $4.8505 E+01$ & 0.027650 & & \\
\hline Th 231 & 231.0363 & $2.552 E+01$ & hour & $9.19 E+04$ & $5.3151 E+05$ & $1.8814 \mathrm{E}-06$ & 0.001210 & & \\
\hline Th 232 & 232.0381 & $1.405 E+10$ & year & $4.43 E+17$ & 1.0965E-07 & $9.1196 E+06$ & 0.023750 & & \\
\hline Th 233 & 233.0416 & $2.230 E+01$ & minute & $1.34 E+03$ & $3.6182 E+07$ & $2.7638 \mathrm{E}-08$ & & & \\
\hline Th 234 & 234.0436 & $2.410 \mathrm{E}+0.1$ & day & $2.08 \mathrm{E}+06$ & $2.3150 E+04$ & $4.3197 \mathrm{E}-05$ & 0.000149 & & \\
\hline Th 235 & 235.0475 & $7.100 E+00$ & minute & $4.26 E+02$ & $1.1267 \mathrm{E}+08$ & $8.8754 \mathrm{E}-09$ & & & \\
\hline Th 236 & 236.0497 & $3.750 E+01$ & minute & $2.25 \mathrm{E}+03$ & $2.1242 E+07$ & $4.7077 \mathrm{E}-08$ & & & \\
\hline $\mathrm{Pa} 214$ & 214.0207 & $1.700 E-02$ & second & 1.70E-02 & $3.1008 E+12$ & $3.2250 \mathrm{E}-13$ & & & \\
\hline Pa 215 & 215.0191 & $1.400 \mathrm{E}-02$ & second & $1.40 \mathrm{E}-02$ & $3.7478 \mathrm{E}+12$ & $2.6683 E-13$ & & & \\
\hline $\mathrm{Pa} 216$ & 216.0191 & $2.000 \mathrm{E}-01$ & second & 2.00E-01 & $2.6113 E+11$ & 3.8295E-12 & & & \\
\hline $\mathrm{Pa} 217$ & 217.0183 & $4.900 \mathrm{E}-03$ & second & $4.90 \mathrm{E}-03$ & $1.0609 E+13$ & $9.4258 E-14$ & & & \\
\hline $\mathrm{Pa} 217 \mathrm{~m}$ & 217.0183 & $1.600 \mathrm{E}-03$ & second & 1.60E-03 & $3.2491 \mathrm{E}+13$ & $3.0778 \mathrm{E}-14$ & & & \\
\hline Pa 218 & 218.0200 & $1.200 \mathrm{E}-04$ & second & $1.20 \mathrm{E}-04$ & $4.3122 \mathrm{E}+14$ & $2.3190 \mathrm{E}-15$ & & & \\
\hline $\mathrm{Pa} 219$ & 219.0199 & $5.300 \mathrm{E}-08$ & second & $5.30 \mathrm{E}-08$ & $9.7189 E+17$ & $1.0289 \mathrm{E}-18$ & & & \\
\hline $\mathrm{Pa} 221$ & 221.0219 & $5.900 \mathrm{E}-06$ & second & $5.90 \mathrm{E}-06$ & $8.6515 E+15$ & $1.1559 E-16$ & & & \\
\hline $\mathrm{Pa} 222$ & 222.0237 & $2.900 \mathrm{E}-03$ & second & $2.90 \mathrm{E}-03$ & $1.7522 \mathrm{E}+13$ & $5.7072 \mathrm{E}-14$ & & & \\
\hline $\mathrm{Pa} 223$ & 223.0240 & $6.500 \mathrm{E}-03$ & second & $6.50 \mathrm{E}-03$ & $7.7824 E+12$ & $1.2850 \mathrm{E}-13$ & & & \\
\hline $\mathrm{Pa} 224$ & 224.0256 & $9.500 \mathrm{E}-01$ & second & $9.50 \mathrm{E}-01$ & $5.3010 \mathrm{E}+10$ & $1.8864 \mathrm{E}-11$ & & & \\
\hline
\end{tabular}


Radionuclide Specific Data

\begin{tabular}{|c|c|c|c|c|c|c|c|c|c|}
\hline Isotope & $\begin{array}{l}\text { Atomic } \\
\text { Mass }\end{array}$ & Halflife & $\begin{array}{l}\text { Time } \\
\text { Units }\end{array}$ & $\mathfrak{t}_{1 / 2}, \mathrm{sec}$ & Curies/gram & Grams/Curie & $\begin{array}{c}\text { Watts/Ci } \\
\text { (Ref.3) }\end{array}$ & \begin{tabular}{c|} 
Individual $^{1}$ \\
Fission \\
Product Yield \\
(Ref.4)
\end{tabular} & $\begin{array}{l}\text { Cumulative }^{1} \\
\text { Fission Product } \\
\text { Yield (Ref.4) }\end{array}$ \\
\hline Pa 225 & 225.0261 & $1.700 \mathrm{E}+00$ & second & $1.70 E+00$ & $2.9491 \mathrm{E}+10$ & $3.3908 \mathrm{E}-11$ & & & \\
\hline $\mathrm{Pa} 226$ & 226.0279 & $1.800 \mathrm{E}+00$ & minute & $1.08 \mathrm{E}+02$ & $4.6216 E+08$ & $2.1638 \mathrm{E}-09$ & & & \\
\hline $\mathrm{Pa} 227$ & 227.0288 & $3.830 E+01$ & minute & $2.30 E+03$ & $2.1624 E+07$ & $4.6244 E-08$ & & & \\
\hline $\mathrm{Pa} 228$ & 228.0310 & $2.200 E+01$ & hour & $7.92 E+04$ & $6.2468 \mathrm{E}+05$ & $1.6008 \mathrm{E}-06$ & & & \\
\hline $\mathrm{Pa} 229$ & 229.0321 & $1.500 \mathrm{E}+00$ & day & $1.30 \mathrm{E}+05$ & $3.8008 \mathrm{E}+05$ & $2.6310 E-06$ & & & \\
\hline $\mathrm{Pa} 230$ & 230.0345 & $1.740 \mathrm{E}+01$ & day & $1.50 \mathrm{E}+06$ & $3.2623 E+04$ & $3.0653 \mathrm{E}-05$ & & & \\
\hline Pa 231 & 231.0359 & $3.276 E+04$ & year & $1.03 E+12$ & $4.7232 \mathrm{E}-02$ & $2.1172 E+01$ & 0.029700 & & \\
\hline Pa 232 & 232.0386 & $1.310 E+00$ & day & $1.13 E+05$ & $4.2957 E+05$ & $2.3279 E-06$ & & & \\
\hline $\mathrm{Pa} 233$ & 233.0402 & $2.697 E+01$ & day & $2.33 E+06$ & $2.0778 E+04$ & $4.8128 \mathrm{E}-05$ & 0.002360 & & \\
\hline Pa 234 & 234.0433 & $6.700 E+00$ & hour & $2.41 \mathrm{E}+04$ & $1.9985 E+06$ & $5.0038 \mathrm{E}-07$ & & & \\
\hline $\mathrm{Pa} 234 \mathrm{~m}$ & 234.0433 & $1.170 \mathrm{E}+00$ & minute & $7.02 E+01$ & $6.8666 \mathrm{E}+08$ & 1.4563E-09 & 0.004950 & & \\
\hline $\mathrm{Pa} 235$ & 235.0454 & $2.450 E+01$ & minute & $1.47 \mathrm{E}+03$ & $3.2652 E+07$ & $3.0626 \mathrm{E}-08$ & & & \\
\hline $\mathrm{Pa} 236$ & 236.0487 & $9.100 E+00$ & minute & $5.46 \mathrm{E}+02$ & $8.7535 \mathrm{E}+07$ & $1.1424 \mathrm{E}-08$ & & & \\
\hline Pa 237 & 237.0511 & $8.700 E+00$ & minute & $5.22 E+02$ & $9.1173 E+07$ & $1.0968 \mathrm{E}-08$ & & & \\
\hline $\mathrm{Pa} 238$ & 238.0545 & $2.300 E+00$ & minute & $1.38 \mathrm{E}+02$ & $3.4342 E+08$ & $2.9119 \mathrm{E}-09$ & & & \\
\hline U218 & 218.0200 & $1.500 \mathrm{E}-03$ & second & $1.50 \mathrm{E}-03$ & $3.4498 \mathrm{E}+13$ & $2.8988 \mathrm{E}-14$ & & & \\
\hline U219 & 219.0249 & $4.200 \mathrm{E}-05$ & second & $4.20 \mathrm{E}-05$ & $1.2264 \mathrm{E}+15$ & $8.1539 \mathrm{E}-16$ & & & \\
\hline $\mathrm{U}-222$ & 222.0261 & $1.000 \mathrm{E}-06$ & second & 1.00E-06 & $5.0813 E+16$ & $1.9680 \mathrm{E}-17$ & & & \\
\hline $\mathrm{U}-223$ & 223.0277 & $1.800 \mathrm{E}-05$ & second & $1.80 \mathrm{E}-05$ & $2.8103 \mathrm{E}+15$ & $3.5584 \mathrm{E}-16$ & & & \\
\hline U 224 & 224.0276 & $9.000 \mathrm{E}-04$ & second & $9.00 \mathrm{E}-04$ & $5.5954 E+13$ & $1.7872 E-14$ & & & \\
\hline U 225 & 225.0261 & $9.500 \mathrm{E}-02$ & second & $9.50 \mathrm{E}-02$ & $5.2774 \mathrm{E}+11$ & $1.8949 \mathrm{E}-12$ & & & \\
\hline U226 & 226.0293 & $5.000 \mathrm{E}-01$ & second & $5.00 \mathrm{E}-01$ & $9.9826 \mathrm{E}+10$ & $1.0017 \mathrm{E}-11$ & & & \\
\hline U227 & 227.0311 & $1.100 E+00$ & minute & $6.60 E+01$ & $7.5292 \mathrm{E}+08$ & $1.3282 \mathrm{E}-09$ & & & \\
\hline U 228 & 228.0314 & $9.100 E+00$ & minute & $5.46 \mathrm{E}+02$ & $9.0613 \mathrm{E}+07$ & $1.1036 \mathrm{E}-08$ & & & \\
\hline$\cup 229$ & 229.0335 & $5.800 E+01$ & minute & $3.48 E+03$ & $1.4155 \mathrm{E}+07$ & $7.0648 \mathrm{E}-08$ & & & \\
\hline$\cup 230$ & 230.0339 & $2.080 E+01$ & day & $1.80 E+06$ & $2.7290 \mathrm{E}+04$ & $3.6643 \mathrm{E}-05$ & & & \\
\hline$\cup 231$ & 231.0363 & $4.200 \mathrm{E}+00$ & day & $3.63 E+05$ & $1.3457 \mathrm{E}+05$ & $7.4313 \mathrm{E}-06$ & & & \\
\hline$\cup 232$ & 232.0371 & $6.890 E+01$ & year & $2.17 E+09$ & $2.2361 E+01$ & $4.4721 \mathrm{E}-02$ & 0.031460 & & \\
\hline U233 & 233.0396 & $1.592 E+05$ & year & $5.02 E+12$ & $9.6358 \mathrm{E}-03$ & $1.0378 E+02$ & 0.028570 & & \\
\hline U234 & 234.0409 & $2.455 E+05$ & year & $7.75 \mathrm{E}+12$ & $6.2218 \mathrm{E}-03$ & $1.6072 E+02$ & 0.028290 & & \\
\hline U235 & 235.0439 & $7.038 E+08$ & year & $2.22 E+16$ & $2.1610 \mathrm{E}-06$ & $4.6274 E+05$ & 0.027130 & & \\
\hline U $235 \mathrm{~m}$ & 235.0439 & $2.500 E+01$ & minute & $1.50 E+03$ & $3.1999 \mathrm{E}+07$ & $3.1251 E-08$ & & & \\
\hline U 236 & 236.0456 & $2.342 E+07$ & year & $7.39 \mathrm{E}+14$ & $6.4666 \mathrm{E}-05$ & $1.5464 \mathrm{E}+04$ & 0.026620 & & \\
\hline U $236 \mathrm{~m}$ & 236.0456 & $1.210 \mathrm{E}-07$ & second & 1.21E-07 & $3.9500 \mathrm{E}+17$ & $2.5317 \mathrm{E}-18$ & & & \\
\hline U237 & 237.0487 & $6.750 E+00$ & day & $5.83 E+05$ & $8.1606 E+04$ & $1.2254 \mathrm{E}-05$ & & & \\
\hline
\end{tabular}

WSRC-TR-99-00344

October, 1999

page 72 


\begin{tabular}{|c|c|c|c|c|c|c|c|c|c|}
\hline Isotope & $\begin{array}{l}\text { Atomic } \\
\text { Mass }\end{array}$ & Halflife & $\begin{array}{l}\text { Time } \\
\text { Units }\end{array}$ & $t_{12}, \sec$ & Curies/gram & Grams/ Curie & $\begin{array}{c}\text { Watts/Ci } \\
\text { (Ref.3) }\end{array}$ & $\begin{array}{c}\text { Individual }^{1} \\
\text { Fission } \\
\text { Product Yield } \\
\text { (Ref.4) } \\
\end{array}$ & $\begin{array}{c}\text { Cumulative' } \\
\text { Fission Product } \\
\text { Yield (Ref.4) }\end{array}$ \\
\hline U 238 & 238.0508 & $4.468 \mathrm{E}+09$ & year & $1.41 \mathrm{E}+17$ & $3.3611 E-07$ & $2.9752 E+06$ & 0.024920 & & \\
\hline U 239 & 239.0543 & $2.345 E+01$ & minute & $1.41 E+03$ & $3.3542 E+07$ & $2.9814 \mathrm{E}-08$ & & & \\
\hline U 240 & 240.0566 & $1.410 \mathrm{E}+01$ & hour & $5.08 E+04$ & $9.2585 \mathrm{E}+05$ & $1.0801 \mathrm{E}-06$ & & & \\
\hline $\mathrm{U}-242$ & 242.0629 & $1.680 \mathrm{E}+01$ & minute & $1.01 E+03$ & $4.6237 \mathrm{E}+07$ & $2.1628 \mathrm{E}-08$ & & & \\
\hline Np 225 & 225.0339 & $?$ & second & $?$ & & & & & \\
\hline Np 226 & 226.0351 & $3.100 \mathrm{E}-02$ & second & $3.10 \mathrm{E}-02$ & $1.6100 \mathrm{E}+12$ & $6.2110 \mathrm{E}-13$ & & & \\
\hline Np 227 & 227.0350 & $5.100 \mathrm{E}-01$ & second & $5.10 \mathrm{E}-01$ & $9.7435 \mathrm{E}+10$ & $1.0263 \mathrm{E}-11$ & & & \\
\hline Np 228 & 228.0387 & $5.800 E+01$ & second & $5.80 E+01$ & $8.5298 E+08$ & $1.1724 E-09$ & & & \\
\hline Np 229 & 229.0362 & $4.000 E+00$ & minute & $2.40 E+02$ & $2.0524 E+08$ & $4.8724 \mathrm{E}-09$ & & & \\
\hline $\mathrm{Np} 230$ & 230.0378 & $4.600 \mathrm{E}+00$ & minute & $2.76 E+02$ & $1.7769 \mathrm{E}+08$ & $5.6277 \mathrm{E}-09$ & & & \\
\hline Np 231 & 231.0382 & $4.880 E+01$ & minute & $2.93 E+03$ & $1.6677 E+07$ & $5.9962 \mathrm{E}-08$ & & & \\
\hline $\mathrm{Np} 232$ & 232.0401 & $1.470 \mathrm{E}+01$ & minute & $8.82 E+02$ & $5.5125 \mathrm{E}+07$ & $1.8141 \mathrm{E}-08$ & & & \\
\hline $\mathrm{Np} 233$ & 233.0407 & $3.620 E+01$ & minute & $2.17 E+03$ & $2.2289 \mathrm{E}+07$ & $4.4866 \mathrm{E}-08$ & & & \\
\hline $\mathrm{Np} 234$ & 234.0429 & $4.400 E+00$ & day & $3.80 E+05$ & $1.2680 E+05$ & $7.8865 \mathrm{E}-06$ & & & \\
\hline Np 235 & 235.0441 & $3.961 E+02$ & day & $3.42 E+07$ & $1.4025 \mathrm{E}+03$ & $7.1300 \mathrm{E}-04$ & & & \\
\hline Np 236. & 236.0466 & $1.540 E+05$ & year & $4.86 \mathrm{E}+12$ & $9.8343 \mathrm{E}-03$ & $1.0169 \mathrm{E}+02$ & 0.002000 & & \\
\hline $\mathrm{Np} \mathrm{236m}$ & 236.0466 & $2.250 E+01$ & hour & $8.10 E+04$ & $5.9006 E+05$ & $1.6948 \mathrm{E}-06$ & & & \\
\hline Np 237 & 237.0482 & $2.140 E+06$ & year & $6.75 E+13$ & $7.0471 \mathrm{E}-04$ & $1.4190 \mathrm{E}+03$ & 0.028790 & & \\
\hline $\mathrm{Np} 237 \mathrm{~m}$ & 237.0482 & 4.500 E-08 & second & $4.50 \mathrm{E}-08$ & $1.0576 E+18$ & $9.4552 \mathrm{E}-19$ & & & \\
\hline Np 238 & 238.0509 & $2.117 E+00$ & day & $1.83 E+05$ & $2.5910 E+05$ & $3.8595 \mathrm{E}-06$ & & & \\
\hline Np 239 & 239.0529 & $2.357 \mathrm{E}+00$ & day & $2.04 E+05$ & $2.3179 E+05$ & $4.3142 \mathrm{E}-06$ & 0.002530 & & \\
\hline $\mathrm{Np} 240$ & 240.0562 & $6.190 \mathrm{E}+01$ & minute & $3.71 \mathrm{E}+03$ & $1.2654 \mathrm{E}+07$ & $7.9028 \mathrm{E}-08$ & & & \\
\hline $\mathrm{Np} \mathrm{240m}$ & 240.0562 & $7.220 \mathrm{E}+00$ & minute & $4.33 E+02$ & $1.0849 E+08$ & $9.2177 \mathrm{E}-09$ & & & \\
\hline Np 241 & 241.0582 & $1.390 E+01$ & minute & $8.34 E+02$ & $5.6116 E+07$ & $1.7820 \mathrm{E}-08$ & & & \\
\hline $\mathrm{Np} 242$ & 242.0616 & $5.500 E+00$ & minute & $3.30 \mathrm{E}+02$ & $1.4123 E+08$ & $7.0805 E-09$ & & & \\
\hline $\mathrm{Np} 242 \mathrm{~m}$ & 242.0616 & $2.200 E+00$ & minute & $1.32 E+02$ & $3.5308 \mathrm{E}+08$ & $2.8322 E-09$ & & & \\
\hline $\mathrm{Np} 243$ & 243.0643 & $1.850 \mathrm{E}+00$ & minute & $1.11 \mathrm{E}+02$ & $4.1815 \mathrm{E}+08$ & $2.3915 \mathrm{E}-09$ & & & \\
\hline $\mathrm{Np} 244$ & 244.0679 & $2.290 E+00$ & minute & $1.37 \mathrm{E}+02$ & $3.3642 E+08$ & $2.9725 \mathrm{E}-09$ & & & \\
\hline Pu 231 & 231.0413 & $?$ & minute & $?$ & $?$ & \#VALUE! & & & \\
\hline Pu 232 & 232.0412 & $3.410 \mathrm{E}+01$ & minute & $2.05 E+03$ & $2.3763 E+07$ & $4.2082 \mathrm{E}-08$ & & & \\
\hline Pu 233 & 233.0430 & $2.090 E+01$ & minute & $1.25 E+03$ & $3.8605 \mathrm{E}+07$ & $2.5903 \mathrm{E}-08$ & & & \\
\hline Pu 234 & 234.0433 & $8.800 E+00$ & hour & $3.17 \mathrm{E}+04$ & $1.5216 \mathrm{E}+06$ & $6.5721 \mathrm{E}-07$ & & & \\
\hline Pu 235 & 235.0453 & $2.530 \mathrm{E}+01$ & minute & $1.52 E+03$ & $3.1619 \mathrm{E}+07$ & $3.1626 \mathrm{E}-08$ & & & \\
\hline $\mathrm{Pu} 236$ & 236.0460 & $2.858 \mathrm{E}+00$ & year & $9.02 E+07$ & $5.2991 \mathrm{E}+02$ & $1.8871 \mathrm{E}-03$ & 0.034180 & & \\
\hline $\mathrm{Pu} 237$ & 237.0484 & $4.520 E+01$ & day & $3.91 E+06$ & $1.2187 \mathrm{E}+04$ & $8.2056 E-05$ & & & \\
\hline
\end{tabular}

WSRC-TR-99-00344

October, 1999

page 73 
Radionuclide Specific Data

\begin{tabular}{|c|c|c|c|c|c|c|c|c|c|}
\hline Isotope & $\begin{array}{c}\text { Atomic } \\
\text { Mass }\end{array}$ & Halflife & $\begin{array}{l}\text { Time } \\
\text { Units }\end{array}$ & $\mathrm{t}_{1 / 2}, \mathrm{sec}$ & Curies/gram & Grams/Curie & $\begin{array}{c}\text { Watts/Ci } \\
\text { (Ref.3) }\end{array}$ & $\begin{array}{c}\text { Individual }^{1} \\
\text { Fission } \\
\text { Product Yield } \\
\text { (Ref.4) }\end{array}$ & $\begin{array}{c}\text { Cumulative } \\
\text { Fission Product } \\
\text { Yield (Ref.4) }\end{array}$ \\
\hline $\mathrm{Pu} 237 \mathrm{~m}$ & 237.0484 & $1.800 \mathrm{E}-01$ & second & 1.80 E-01 & $2.6440 \mathrm{E}+11$ & $3.7821 \mathrm{E}-12$ & & & \\
\hline Pu $237 \mathrm{~m} 2$ & 237.0484 & $8.500 \mathrm{E}-08$ & second & $8.50 E-08$ & $5.5991 E+17$ & $1.7860 \mathrm{E}-18$ & & & \\
\hline Pu $237 \mathrm{~m} 3$ & 237.0484 & $1.100 \mathrm{E}-06$ & second & $1.10 \mathrm{E}-06$ & $4.3266 E+16$ & $2.3113 \mathrm{E}-17$ & & & \\
\hline Pu 238 & 238.0496 & $8.774 E+01$ & year & $2.77 E+09$ & $1.7116 \mathrm{E}+01$ & $5.8426 \mathrm{E}-02$ & 0.032593 & & \\
\hline Pu 238m & 238.0496 & $6.000 \mathrm{E}-10$ & second & $6.00 \mathrm{E}-10$ & $7.8987 \mathrm{E}+19$ & $1.2660 \mathrm{E}-20$ & & & \\
\hline Pu $238 \mathrm{~m} 2$ & 238.0496 & $6.000 \mathrm{E}-09$ & second & $6.00 \mathrm{E}-09$ & $7.8987 \mathrm{E}+18$ & $1.2660 \mathrm{E}-19$ & & & \\
\hline Pu 239 & 239.0522 & $2.411 E+04$ & year & $7.61 \mathrm{E}+11$ & $6.2026 \mathrm{E}-02$ & $1.6122 \mathrm{E}+01$ & 0.030240 & & \\
\hline $\mathrm{Pu} 240$ & 240.0538 & $6.564 E+03$ & year & $2.07 E+11$ & $2.2687 E-01$ & $4.4077 \mathrm{E}+00$ & 0.030560 & & \\
\hline Pu 240m & 240.0538 & $3.700 \mathrm{E}-09$ & second & $3.70 \mathrm{E}-09$ & $1.2702 E+19$ & $7.8729 \mathrm{E}-20$ & & & \\
\hline $\mathrm{Pu} 241$ & 241.0568 & $1.435 E+01$ & year & $4.53 E+08$ & $1.0334 E+02$ & $9.6763 \mathrm{E}-03$ & 0.000032 & & \\
\hline Pu 241m & 241.0568 & $2.100 \mathrm{E}-05$ & second & $2.10 \mathrm{E}-05$ & $2.2286 E+15$ & $4.4871 \mathrm{E}-16$ & & & \\
\hline$P u 242$ & 242.0587 & $3.733 E+05$ & year & $1.18 \mathrm{E}+13$ & $3.9562 \mathrm{E}-03$ & $2.5277 \mathrm{E}+02$ & 0.029040 & & \\
\hline$P \cup 243$ & 243.0620 & $4.956 \mathrm{E}+00$ & hour & $1.78 E+04$ & $2.6015 E+06$ & $3.8439 \mathrm{E}-07$ & & & \\
\hline Pu 244 & 244.0642 & $8.080 E+07$ & year & $2.55 E+15$ & $1.8128 \mathrm{E}-05$ & $5.5164 E+04$ & 0.027120 & & \\
\hline Pu 245 & 245.0677 & $1.050 \mathrm{E}+01$ & hour & $3.78 E+04$ & $1.2179 \mathrm{E}+06$ & $8.2111 \mathrm{E}-07$ & & & \\
\hline PU246 & 246.0702 & $1.084 E+01$ & day & $9.37 \mathrm{E}+05$ & $4.8952 E+04$ & $2.0428 \mathrm{E}-05$ & & & \\
\hline$P \cup 247$ & 247.0741 & $2.270 E+00$ & day & $1.96 E+05$ & $2.3281 E+05$ & $4.2953 \mathrm{E}-06$ & & & \\
\hline Am 232 & 232.0466 & $7.900 \mathrm{E}+01$ & second & $7.90 \mathrm{E}+01$ & $6.1542 E+08$ & $1.6249 \mathrm{E}-09$ & & & \\
\hline Am 234 & 234.0478 & $2.320 \mathrm{E}+00$ & minute & $1.39 E+02$ & $3.4628 \mathrm{E}+08$ & $2.8878 E-09$ & & & \\
\hline $\operatorname{Am~237}$ & 237.0500 & $7.300 \mathrm{E}+01$ & minute & $4.38 E+03$ & $1.0866 \mathrm{E}+07$ & $9.2032 \mathrm{E}-08$ & & & \\
\hline Am $237 \mathrm{~m}$ & 237.0500 & $5.000 \mathrm{E}-09$ & second & $5.00 \mathrm{E}-09$ & $9.5185 \mathrm{E}+18$ & $1.0506 \mathrm{E}-19$ & & & \\
\hline Am 238 & 238.0520 & $9.800 \mathrm{E}+01$ & minute & $5.88 \mathrm{E}+03$ & $8.0599 E+06$ & $1.2407 \mathrm{E}-07$ & & & \\
\hline Am 239 & 239.0530 & $1.190 \mathrm{E}+01$ & hour. & $4.28 E+04$ & $1.1016 \mathrm{E}+06$ & $9.0775 \mathrm{E}-07$ & & & \\
\hline Am 240 & 240.0553 & $5.080 \mathrm{E}+01$ & hour & $1.83 E+05$ & $2.5698 \mathrm{E}+05$ & $3.8914 \mathrm{E}-06$ & & & \\
\hline Am $240 \mathrm{~m}$ & 240.0553 & $9.420 E-04$ & second & $9.42 \mathrm{E}-04$ & $4.9890 E+13$ & $2.0044 \mathrm{E}-14$ & & & \\
\hline $\operatorname{Am~241}$ & 241.0568 & $4.322 E+02$ & year & $1.36 E+10$ & $3.4313 \mathrm{E}+00$ & $2.9144 E-01$ & 0.032830 & & \\
\hline Am $241 \mathrm{~m}$ & 241.0568 & $1.200 \mathrm{E}-06$ & second & $1.20 \mathrm{E}-06$ & $3.9001 \mathrm{E}+16$ & $2.5640 \mathrm{E}-17$ & & & \\
\hline $\operatorname{Am~} 242$ & 242.0595 & $1.602 E+01$ & hour & $5.77 E+04$ & $8.0814 E+05$ & $1.2374 \mathrm{E}-06$ & 0.001160 & & \\
\hline Am 242m & 242.0595 & $1.410 \mathrm{E}+02$ & year & $4.45 \mathrm{E}+09$ & $1.0474 E+01$ & $9.5473 \mathrm{E}-02$ & 0.000405 & & \\
\hline Am $242 \mathrm{~m} 2$ & 242.0595 & $1.400 \mathrm{E}-02$ & second & $1.40 \mathrm{E}-02$ & $3.3291 \mathrm{E}+12$ & $3.0038 E-13$ & & & \\
\hline Am 243 & 243.0614 & $7.370 E+03$ & year & $2.33 E+11$ & $1.9956 \mathrm{E}-01$ & $5.0110 \mathrm{E}+00$ & 0.031496 & & \\
\hline Am 244 & 244.0643 & $1.010 \mathrm{E}+01$ & hour & $3.64 E+04$ & $1.2713 \mathrm{E}+06$ & $7.8660 \mathrm{E}-07$ & & & \\
\hline Am $244 m$ & 244.0643 & $6.500 \mathrm{E}-06$ & second & $6.50 \mathrm{E}-06$ & $7.1115 \mathrm{E}+15$ & $1.4062 E-16$ & & & \\
\hline Am 244m2 & 244.0643 & $2.600 \mathrm{E}+01$ & minute & $1.56 \mathrm{E}+03$ & $2.9631 \mathrm{E}+07$ & $3.3748 \mathrm{E}-08$ & & & \\
\hline Am 245 & 245.0664 & $2.050 \mathrm{E}+00$ & hour & $7.38 \mathrm{E}+03$ & $6.2379 \mathrm{E}+06$ & $1.6031 \mathrm{E}-07$ & & & \\
\hline
\end{tabular}

WSRC-TR-99-00344

October, 1999

page 74 


\begin{tabular}{|c|c|c|c|c|c|c|c|c|c|}
\hline Isotope & $\begin{array}{c}\text { Atomic } \\
\text { Mass }\end{array}$ & Halflife & $\begin{array}{l}\text { Time } \\
\text { Units }\end{array}$ & $t_{1 / 2}, \sec$ & Curies/ gram & Grams/ Curie & $\begin{array}{c}\text { Watts/Ci } \\
\text { (Ref.3) }\end{array}$ & $\begin{array}{l}\text { Individual }^{1} \\
\text { Fission } \\
\text { Product Yield } \\
\text { (Ref.4) }\end{array}$ & $\begin{array}{c}\text { Cumulative' } \\
\text { Fission Product } \\
\text { Yield (Ref.4) }\end{array}$ \\
\hline Am 246 & 246.0698 & $3.900 E+01$ & minute & $2.34 \mathrm{E}+03$ & $1.9593 E+07$ & $5.1038 \mathrm{E}-08$ & & & \\
\hline Am 246m & 246.0698 & $2.500 E+01$ & minute & $1.50 \mathrm{E}+03$ & $3.0565 E+07$ & $3.2717 \mathrm{E}-08$ & & & \\
\hline Am 247 & 247.0721 & $2.300 E+01$ & minute & $1.38 \mathrm{E}+03$ & $3.3088 E+07$ & $3.0222 \mathrm{E}-08$ & & & \\
\hline $\mathrm{Cm} 238$ & 238.0530 & $2.400 E+00$ & hour & $8.64 \mathrm{E}+03$ & $5.4852 E+06$ & $1.8231 E-07$ & & & \\
\hline $\mathrm{Cm} 239$ & 239.0550 & $2.900 E+00$ & hour & $1.04 \mathrm{E}+04$ & $4.5204 E+06$ & $2.2122 E-07$ & & & \\
\hline $\mathrm{Cm} 240$ & 240.0555 & $2.700 E+01$ & day & $2.33 E+06$ & $2.0146 E+04$ & $4.9638 E-05$ & & & \\
\hline $\mathrm{Cm} 240 \mathrm{~m}$ & 240.0555 & $1.000 \mathrm{E}-11$ & second & $1.00 \mathrm{E}-11$ & $4.6996 E+21$ & $2.1278 E-22$ & & & \\
\hline $\mathrm{Cm} 240 \mathrm{~m} 2$ & 240.0555 & $5.500 \mathrm{E}-08$ & second & $5.50 E-08$ & $8.5448 E+17$ & $1.1703 E-18$ & & & \\
\hline $\mathrm{Cm} 241$ & 241.0576 & $3.280 E+01$ & day & $2.83 E+06$ & $1.6515 E+04$ & $6.0552 E-05$ & & & \\
\hline $\mathrm{Cm} 242$ & 242.0588 & $1.628 \mathrm{E}+02$ & day & $1.41 E+07$ & $3.3135 E+03$ & 3.0180 E-04 & 0.035886 & & \\
\hline $\mathrm{Cm} 242 \mathrm{~m}$ & 242.0588 & 1.800E-07 & second & $1.80 \mathrm{E}-07$ & $2.5893 E+17$ & $3.8620 \mathrm{E}-18$ & & & \\
\hline $\mathrm{Cm} 243$ & 243.0614 & $2.910 \mathrm{E}+01$ & year & $9.18 E+08$ & $5.0542 E+01$ & $1.9786 \mathrm{E}-02$ & 0.036050 & & \\
\hline Cm 244 & 244.0627 & $1.810 \mathrm{E}+01$ & year & $5.71 \mathrm{E}+08$ & $8.0925 E+01$ & $1.2357 \mathrm{E}-02$ & 0.034370 & & \\
\hline $\mathrm{Cm} \mathrm{244m}$ & 244.0627 & $3.400 \mathrm{E}-02$ & second & $3.40 \mathrm{E}-02$ & $1.3596 \mathrm{E}+12$ & $7.3554 \mathrm{E}-13$ & & & \\
\hline $\mathrm{Cm} \mathrm{245}$ & 245.0655 & $8.500 \mathrm{E}+03$ & year & $2.68 \mathrm{E}+11$ & $1.7162 \mathrm{E}-01$ & $5.8269 E+00$ & 0.033290 & & \\
\hline $\mathrm{Cm} 246$ & 246.0672 & $4.730 E+03$ & year & $1.49 E+11$ & $3.0715 \mathrm{E}-01$ & $3.2558 \mathrm{E}+00$ & 0.031920 & & \\
\hline $\mathrm{Cm} 247$ & 247.0703 & $1.560 E+07$ & year & $4.92 E+14$ & 9.2750 E-05 & $1.0782 E+04$ & 0.031190 & & \\
\hline $\mathrm{Cm} 248$ & 248.0723 & $3.400 E+05$ & year & $1.07 E+13$ & $4.2384 \mathrm{E}-03$ & $2.3594 E+02$ & 0.027577 & & \\
\hline $\mathrm{Cm} 249$ & 249.0759 & $6.415 E+01$ & minute & $3.85 \mathrm{E}+03$ & $1.1768 E+07$ & 8.4977E-08 & & & \\
\hline $\mathrm{Cm} 250$ & 250.0784 & $9.700 \mathrm{E}+03$ & year & $3.06 \mathrm{E}+11$ & 1.4737E-01 & $6.7856 \mathrm{E}+00$ & & & \\
\hline $\mathrm{Cm} 251$ & 251.0823 & $1.680 \mathrm{E}+01$ & minute & $1.01 \mathrm{E}+03$ & $4.4576 E+07$ & $2.2434 E-08$ & & & \\
\hline $\mathrm{Cm} 252$ & 252.0849 & $2.000 E+00$ & day & $1.73 E+05$ & $2.5899 E+05$ & $3.8611 \mathrm{E}-06$ & & & \\
\hline Bk 238 & 238.0583 & $1.440 \mathrm{E}+02$ & second & $1.44 E+02$ & $3.2910 E+08$ & $3.0386 E-09$ & & & \\
\hline Bk 240 & 240.0597 & $4.800 \mathrm{E}+00$ & minute & $2.88 \mathrm{E}+02$ & $1.6318 E+08$ & $6.1282 E-09$ & & & \\
\hline Bk 242 & 242.0621 & $7.000 E+00$ & minute & $4.20 E+02$ & $1.1097 E+08$ & $9.0116 \mathrm{E}-09$ & & & \\
\hline Bk 243 & 243.0630 & $4.500 \mathrm{E}+00$ & hour & $1.62 \mathrm{E}+04$ & $2.8651 \mathrm{E}+06$ & 3.4903E-07 & & & \\
\hline Bk 244 & 244.0652 & $4.350 E+00$ & hour & $1.57 E+04$ & $2.9517 E+06$ & $3.3878 E-07$ & & & \\
\hline Bk 244m & 244.0652 & $8.200 E-07$ & second & $8.20 E-07$ & $5.6371 E+16$ & $1.7740 \mathrm{E}-17$ & & & \\
\hline BK 245 & 245.0664 & $4.940 \mathrm{E}+00$ & day & $4.27 E+05$ & $1.0786 \mathrm{E}+05$ & $9.2715 \mathrm{E}-06$ & & & \\
\hline Bk 246 & 246.0687 & $1.800 \mathrm{E}+00$ & day & $1.56 \mathrm{E}+05$ & $2.9480 E+05$ & $3.3921 \mathrm{E}-06$ & & & \\
\hline Bk 247 & 247.0703 & $1.380 \mathrm{E}+03$ & year & $4.36 \mathrm{E}+10$ & $1.0485 E+00$ & 9.5376E-01 & & & \\
\hline BK 248 & 248.0731 & $9.000 E+00$ & year & $2.84 E+08$ & $1.6012 \mathrm{E}+02$ & $6.2454 E-03$ & & & \\
\hline Bk 248m & 248.0731 & $2.370 \mathrm{E}+01$ & hour & $8.53 E+04$ & $5.3302 E+05$ & $1.8761 \mathrm{E}-06$ & & & \\
\hline Bk 249 & 249.0750 & $3.200 E+02$ & day & $2.76 \mathrm{E}+07$ & $1.6383 E+03$ & $6.1040 E-04$ & & & \\
\hline BK 250 & 250.0783 & $3.217 \mathrm{E}+00$ & hour & $1.16 \mathrm{E}+04$ & $3.8954 \mathrm{E}+06$ & $2.5672 \mathrm{E}-07$ & & & \\
\hline
\end{tabular}

WSRC-TR-99-00344

October, 1999

page 75 
Radionuclide Specific Data

\begin{tabular}{|c|c|c|c|c|c|c|c|c|c|}
\hline |sotope & $\begin{array}{l}\text { Atomic } \\
\text { Mass }\end{array}$ & Halflife & $\begin{array}{l}\text { Time } \\
\text { Units }\end{array}$ & $\mathfrak{t}_{1 / 2}, \sec$ & Curies/gram & Grams/ Curie & $\begin{array}{c}\text { Watts/Ci } \\
\text { (Ref.3) }\end{array}$ & $\begin{array}{c}\text { Individual }^{1} \\
\text { Fission } \\
\text { Product Yield } \\
\text { (Ref.4) }\end{array}$ & $\begin{array}{l}\text { Cumulative' } \\
\text { Fission Product } \\
\text { Yield (Ref.4) }\end{array}$ \\
\hline Bk 251 & 251.0808 & $5.560 E+01$ & minute & $3.34 E+03$ & $1.3469 \mathrm{E}+07$ & $7.4244 E-08$ & & & \\
\hline$\overline{C f 239}$ & 239.0626 & $3.900 \mathrm{E}+01$ & second & $3.90 E+01$ & $1.2100 \mathrm{E}+09$ & $8.2642 \mathrm{E}-10$ & & & \\
\hline Cf.240 & 240.0623 & $1.060 \mathrm{E}+00$ & minute & $6.36 \mathrm{E}+01$ & $7.3892 E+08$ & $1.3533 \mathrm{E}-09$ & & & \\
\hline Cf 241 & 241.0637 & $3.780 E+00$ & minute & $2.27 \mathrm{E}+02$ & $2.0635 E+08$ & $4.8462 \mathrm{E}-09$ & & & \\
\hline Cf 242 & 242.0637 & $3.490 E+00$ & minute & $2.09 E+02$ & $2.2257 E+08$ & $4.4929 \mathrm{E}-09$ & & & \\
\hline Cf 243 & 243.0654 & $1.070 E+01$ & minute & $6.42 E+02$ & $7.2297 E+07$ & $1.3832 E-08$ & & & \\
\hline Cf 244 & 244.0660 & $1.940 E+01$ & minute & $1.16 \mathrm{E}+03$ & $3.9711 \mathrm{E}+07$ & $2.5182 E-08$ & & & \\
\hline Cf 245 & 245.0680 & $4.500 E+01$ & minute & $2.70 \mathrm{E}+03$ & $1.7050 \mathrm{E}+07$ & $5.8651 \mathrm{E}-08$ & & & \\
\hline Cf 246 & 246.0688 & $3.570 E+01$ & hour & $1.29 \mathrm{E}+05$ & $3.5674 E+05$ & $2.8032 E-06$ & & & \\
\hline$\overline{C f 247}$ & 247.0710 & $3.110 E+00$ & hour & $1.12 E+04$ & $4.0784 \mathrm{E}+06$ & $2.4519 \mathrm{E}-07$ & & & \\
\hline Cf 248 & 248.0722 & $3.335 E+02$ & day & $2.88 \mathrm{E}+07$ & $1.5783 \mathrm{E}+03$ & $6.3359 \mathrm{E}-04$ & & & \\
\hline Cf 249 & 249.0748 & $3.510 E+02$ & year & $1.11 \mathrm{E}+10$ & $4.0891 E+00$ & 2.4455E-01 & & & \\
\hline Cf 250 & 250.0764 & $1.308 \mathrm{E}+01$ & year & $4.13 E+08$ & $1.0929 \mathrm{E}+02$ & $9.1500 \mathrm{E}-03$ & & & \\
\hline Cf 251 & 251.0796 & $8.980 E+02$ & year & $2.83 E+10$ & $1.5855 E+00$ & $6.3071 \mathrm{E}-01$ & & & \\
\hline Cf 252 & 252.0816 & $2.645 E+00$ & year & $8.35 E+07$ & $5.3616 E+02$ & $1.8651 \mathrm{E}-03$ & 0.035191 & & \\
\hline Cf 253 & 253.0851 & $1.781 E+01$ & day & $1.54 E+06$ & $2.8969 \mathrm{E}+04$ & $3.4520 \mathrm{E}-05$ & & & \\
\hline Cf 254 & 254.0873 & $6.050 \mathrm{E}+01$ & day & $5.23 E+06$ & $8.4942 E+03$ & $1.1773 \mathrm{E}-04$ & & & \\
\hline$\overline{C f 255}$ & 255.0910 & $8.500 E+01$ & minute & $5.10 \mathrm{E}+03$ & $8.6718 \mathrm{E}+06$ & $1.1532 \mathrm{E}-07$ & & & \\
\hline Cf 256 & 256.1074 & $1.230 E+01$ & minute & $7.38 \mathrm{E}+02$ & $5.9690 \mathrm{E}+07$ & $1.6753 \mathrm{E}-08$ & & & \\
\hline Es 242 & 242.0697 & $7.000 E+00$ & second & $7.00 E+00$ & $6.6579 E+09$ & $1.5020 \mathrm{E}-10$ & & & \\
\hline Es 243 & 243.0696 & $2.100 E+01$ & second & $2.10 E+01$ & $2.2102 \mathrm{E}+09$ & $4.5245 \mathrm{E}-10$ & & & \\
\hline Es 244 & 244.0710 & $3.700 E+01$ & second & $3.70 \mathrm{E}+01$ & $1.2493 E+09$ & $8.0046 E-10$ & & & \\
\hline Es 245 & 245.0713 & $1.100 \mathrm{E}+00$ & minute & $6.60 \mathrm{E}+01$ & $6.9749 E+08$ & $1.4337 \mathrm{E}-09$ & & & \\
\hline Es 246 & 246.0730 & $7.700 \mathrm{E}+00$ & minute & $4.62 E+02$ & $9.9236 \mathrm{E}+07$ & 1.0077 E-08 & & & \\
\hline Es 247 & 247.0737 & $4.550 \mathrm{E}+00$ & minute & $2.73 E+02$ & $1.6726 \mathrm{E}+08$ & $5.9788 \mathrm{E}-09$ & & & \\
\hline Es 248 & 248.0772 & $2.700 E+01$ & minute & $1.62 E+03$ & $2.8072 E+07$ & $3.5623 \mathrm{E}-08$ & & & \\
\hline Es 249 & 249.0764 & $1.022 \mathrm{E}+02$ & minute & $6.13 \mathrm{E}+03$ & $7.3866 \mathrm{E}+06$ & $1.3538 \mathrm{E}-07$ & & & \\
\hline Es 250 & 250.0787 & $8.600 \mathrm{E}+00$ & hour & $3.10 E+04$ & $1.4571 E+06$ & $6.8628 \mathrm{E}-07$ & & & \\
\hline Es 250m & 250.0787 & $2.220 \mathrm{E}+00$ & hour & $7.99 \mathrm{E}+03$ & $5.6447 E+06$ & $1.7716 \mathrm{E}-07$ & & & \\
\hline Es 251 & 251.0800 & $3.300 \mathrm{E}+01$ & hour & $1.19 \mathrm{E}+05$ & $3.7822 E+05$ & $2.6439 E-06$ & & & \\
\hline Es 252 & 252.0830 & $4.717 E+02$ & day & $4.08 \mathrm{E}+07$ & $1.0981 \mathrm{E}+03$ & $9.1064 E-04$ & & & \\
\hline Es 253 & 253.0848 & $2.047 E+01$ & day & $1.77 E+06$ & $2.5205 E+04$ & $3.9675 \mathrm{E}-05$ & & & \\
\hline Es 254 & 254.0880 & $2.757 E+02$ & day & $2.38 \mathrm{E}+07$ & $1.8640 \mathrm{E}+03$ & $5.3649 \mathrm{E}-04$ & & & \\
\hline Es $254 m$ & 254.0880 & $3.930 \mathrm{E}+01$ & hour & $1.41 E+05$ & $3.1383 E+05$ & $3.1864 \mathrm{E}-06$ & & & \\
\hline Es 255 & 255.0903 & $3.980 E+01$ & day & $3.44 E+06$ & $1.2861 E+04$ & $7.7752 \mathrm{E}-05$ & & & \\
\hline
\end{tabular}




\begin{tabular}{|c|c|c|c|c|c|c|c|c|c|}
\hline Isotope & $\begin{array}{c}\text { Atomic } \\
\text { Mass }\end{array}$ & Halflife & $\begin{array}{l}\text { Time } \\
\text { Units }\end{array}$ & $t_{1 / 2}, \sec$ & Curies/ gram & Grams/ Curie & $\begin{array}{c}\text { Watts/Ci } \\
\text { (Ref.3) }\end{array}$ & $\begin{array}{c}\text { Individual }^{1} \\
\text { Fission } \\
\text { Product Yield } \\
\text { (Ref.4) }\end{array}$ & $\begin{array}{c}\text { Cumulative }^{4} \\
\text { Fission Product } \\
\text { Yield (Ref.4) }\end{array}$ \\
\hline Es 256 & 256.0936 & $2.540 E+01$ & minute & $1.52 E+03$ & $2.8906 \mathrm{E}+07$ & $3.4595 \mathrm{E}-08$ & & & \\
\hline \begin{tabular}{|l|} 
Es $256 \mathrm{~m}$ \\
\end{tabular} & 256.0936 & $7.600 E+00$ & hour & $2.74 E+04$ & $1.6101 \mathrm{E}+06$ & $6.2107 \mathrm{E}-07$ & & & \\
\hline $\mathrm{Fm} 242$ & 242.0734 & $8.000 E-04$ & second & $8.00 E-04$ & $5.8256 E+13$ & $1.7166 \mathrm{E}-14$ & & & \\
\hline $\mathrm{Fm} \mathrm{243}$ & 243.0745 & $1.800 \mathrm{E}-01$ & second & $1.80 \mathrm{E}-01$ & $2.5785 E+11$ & $3.8782 \mathrm{E}-12$ & & & \\
\hline $\mathrm{Fm} 244$ & 244.0741 & $3.300 \mathrm{E}-03$ & second & $3.30 \mathrm{E}-03$ & $1.4007 \mathrm{E}+13$ & $7.1394 \mathrm{E}-14$ & & & \\
\hline$\overline{F m ~ 245}$ & 245.0754 & $4.200 E+00$ & second & $4.20 E+00$ & $1.0960 \mathrm{E}+10$ & $9.1237 \mathrm{E}-11$ & & & \\
\hline Fm 246 & 246.0753 & $1.100 E+00$ & second & $1.10 E+00$ & $4.1679 E+10$ & $2.3993 \mathrm{E}-11$ & & & \\
\hline Fm 247 & 247.0768 & $3.500 E+01$ & second & $3.50 E+01$ & $1.3046 \mathrm{E}+09$ & $7.6652 \mathrm{E}-10$ & & & \\
\hline $\mathrm{Fm} 247 \mathrm{~m}$ & 247.0768 & $9.200 E+00$ & second & $9.20 E+00$ & $4.9631 \mathrm{E}+09$ & $2.0149 \mathrm{E}-10$ & & & \\
\hline $\mathrm{Fm} \mathrm{248}$ & 248.0772 & $3.600 E+01$ & second & $3.60 E+01$ & $1.2632 E+09$ & $7.9161 \mathrm{E}-10$ & & & \\
\hline $\mathrm{Fm} \mathrm{249}$ & 249.0790 & $2.600 \mathrm{E}+00$ & minute & $1.56 \mathrm{E}+02$ & $2.9035 \mathrm{E}+08$ & $3.4442 E-09$ & & & \\
\hline $\mathrm{Fm} \mathrm{250}$ & 250.0795 & $3.000 E+01$ & minute & $1.80 E+03$ & $2.5063 E+07$ & $3.9900 \mathrm{E}-08$ & & & \\
\hline $\mathrm{Fm} \mathrm{250m}$ & 250.0795 & $1.800 \mathrm{E}+00$ & second & $1.80 \mathrm{E}+00$ & $2.5063 \mathrm{E}+10$ & $3.9900 \mathrm{E}-11$ & & & \\
\hline $\mathrm{Fm} \mathrm{251}$ & 251.0816 & $5.300 E+00$ & hour & $1.91 \mathrm{E}+04$ & $2.3550 \mathrm{E}+06$ & $4.2464 \mathrm{E}-07$ & & & \\
\hline Fm 252 & 252.0825 & $2.539 E+01$ & hour & $9.14 E+04$ & $4.8963 E+05$ & $2.0424 \mathrm{E}-06$ & & & \\
\hline $\mathrm{Fm} 253$ & 253.0852 & $3.000 E+00$ & day & $2.59 \mathrm{E}+05$ & $1.7198 \mathrm{E}+05$ & $5.8147 \mathrm{E}-06$ & & & \\
\hline $\mathrm{Fm} \mathrm{254}$ & 254.0868 & $3.240 E+00$ & hour & $1.17 E+04$ & $3.8067 E+06$ & $2.6270 \mathrm{E}-07$ & & & \\
\hline Fm 255 & 255.0900 & $2.007 E+01$ & hour & $7.23 E+04$ & $6.1212 \mathrm{E}+05$ & $1.6337 \mathrm{E}-06$ & & & \\
\hline Fm 256 & 256.0918 & $1.576 E+02$ & minute & $9.46 E+03$ & $4.6588 \mathrm{E}+06$ & $2.1465 \mathrm{E}-07$ & & & \\
\hline $\mathrm{Fm} 257$ & 257.0951 & $1.005 E+02$ & day & $8.68 \mathrm{E}+06$ & $5.0536 \mathrm{E}+03$ & $1.9788 \mathrm{E}-04$ & & & \\
\hline Fm 258 & 258.0971 & $3.700 \mathrm{E}-04$ & second & $3.70 E-04$ & $1.1814 \mathrm{E}+14$ & $8.4646 \mathrm{E}-15$ & & & \\
\hline $\mathrm{Fm} \mathrm{259}$ & 259.1006 & $1.500 \mathrm{E}+00$ & second & $1.50 \mathrm{E}+00$ & $2.9028 \mathrm{E}+10$ & $3.4450 \mathrm{E}-11$ & & & \\
\hline $\mathrm{Fm}_{\mathrm{m}} 260$ & 260.0000 & $4.000 \mathrm{E}-03$ & second & $4.00 \mathrm{E}-03$ & $1.0848 \mathrm{E}+13$ & $9.2184 \mathrm{E}-14$ & & & \\
\hline$\overline{M d 246}$ & 246.0819 & $1.000 E+00$ & second & $1.00 E+00$ & $4.5846 \mathrm{E}+10$ & $2.1812 \mathrm{E}-11$ & & & \\
\hline Md 247 & 247.0818 & $1.120 E+00$ & second & $1.12 \mathrm{E}+00$ & $4.0768 \mathrm{E}+10$ & $2.4529 \mathrm{E}-11$ & & & \\
\hline Md 248 & 248.0829 & $7.000 \mathrm{E}+00$ & second & $7.00 \mathrm{E}+00$ & $6.4965 E+09$ & $1.5393 \mathrm{E}-10$ & & & \\
\hline$\overline{M d 249}$ & 249.0830 & $2.400 \mathrm{E}+01$ & second & $2.40 E+01$ & $1.8872 E+09$ & $5.2988 \mathrm{E}-10$ & & & \\
\hline$M d 250$ & 250.0845 & $5.200 E+01$ & second & $5.20 \mathrm{E}+01$ & $8.6753 E+08$ & $1.1527 \mathrm{E}-09$ & & & \\
\hline$\overline{M d 251}$ & 251.0849 & $4.000 \mathrm{E}+00$ & minute & $2.40 E+02$ & $1.8722 E+08$ & $5.3414 \mathrm{E}-09$ & & & \\
\hline$\overline{M d 252}$ & 252.0866 & $2.300 \mathrm{E}+00$ & minute & $1.38 \mathrm{E}+02$ & $3.2430 E+08$ & 3.0836 E-09 & & & \\
\hline Md 253 & 253.0873 & $6.000 E+00$ & minute & $3.60 \mathrm{E}+02$ & $1.2382 \mathrm{E}+08$ & $8.0760 \mathrm{E}-09$ & & & \\
\hline Md 254 & 254.0897 & $2.800 \mathrm{E}+01$ & minute & $1.68 E+03$ & $2.6429 \mathrm{E}+07$ & $3.7837 \mathrm{E}-08$ & & & \\
\hline$\overline{M d} 255$ & 255.0911 & $2.700 E+01$ & minute & $1.62 \mathrm{E}+03$ & $2.7300 E+07$ & $3.6630 \mathrm{E}-08$ & & & \\
\hline$\overline{M d 256}$ & 256.0941 & $7.810 \mathrm{E}+01$ & minute & $4.69 E+03$ & $9.4010 \mathrm{E}+06$ & $1.0637 \mathrm{E}-07$ & & & \\
\hline$\overline{M d ~ 257}$ & 257.0955 & $5.300 \mathrm{E}+00$ & hour & $1.91 E+04$ & $2.2999 E+06$ & $4.3481 \mathrm{E}-07$ & & & \\
\hline
\end{tabular}

WSRC-TR-99-00344

October, 1999

page 77 
Radionuclide Specific Data

\begin{tabular}{|c|c|c|c|c|c|c|c|c|c|}
\hline Isotope & $\begin{array}{l}\text { Atomic } \\
\text { Mass }\end{array}$ & Halflife & $\begin{array}{l}\text { Time } \\
\text { Units }\end{array}$ & $\mathbf{t}_{12}, \mathrm{sec}$ & Curies/ gram & Grams/ Curie & $\begin{array}{c}\text { Watts/Ci } \\
\text { (Ref.3) }\end{array}$ & $\begin{array}{c}\text { Individual }^{1} \\
\text { Fission } \\
\text { Product Yield } \\
\text { (Ref.4) }\end{array}$ & $\begin{array}{c}\text { Cumulative }^{1} \\
\text { Fission Product } \\
\text { Yield (Ref.4) }\end{array}$ \\
\hline Md 258 & 258.0984 & $5.150 E+01$ & day & $4.45 E+06$ & $9.8236 \mathrm{E}+03$ & $1.0180 \mathrm{E}-04$ & & & \\
\hline Md 258m & 258.0984 & $5.700 E+01$ & minute & $3.42 E+03$ & $1.2781 \mathrm{E}+07$ & $7.8241 \mathrm{E}-08$ & & & \\
\hline Md 259 & 259.1005 & $9.600 E+01$ & minute & $5.76 E+03$ & $7.5594 E+06$ & $1.3229 \mathrm{E}-07$ & & & \\
\hline Md 260 & 260.1036 & $3.180 E+01$ & day & $2.75 E+06$ & $1.5787 E+04$ & $6.3345 \mathrm{E}-05$ & & & \\
\hline No 250 & 250.0875 & $2.500 \mathrm{E}-04$ & second & $2.50 \mathrm{E}-04$ & $1.8044 E+14$ & $5.5419 \mathrm{E}-15$ & & & \\
\hline No 251 & 251.0890 & $8.000 \mathrm{E}-01$ & second & $8.00 \mathrm{E}-01$ & $5.6164 \mathrm{E}+10$ & $1.7805 E-11$ & & & \\
\hline No 252 & 252.0890 & $2.300 \mathrm{E}+00$ & second & $2.30 \mathrm{E}+00$ & $1.9458 \mathrm{E}+10$ & $5.1393 \mathrm{E}-11$ & & & \\
\hline No 253 & 253.0906 & $1.700 E+00$ & minute & $1.02 E+02$ & $4.3702 E+08$ & $2.2882 \mathrm{E}-09$ & & & \\
\hline No 254 & 254.0909 & $5.500 \mathrm{E}+01$ & second & $5.50 \mathrm{E}+01$ & $8.0728 \mathrm{E}+08$ & $1.2387 \mathrm{E}-09$ & & & \\
\hline No $254 m$ & 254.0909 & $2.800 \mathrm{E}-01$ & second & $2.80 E-01$ & $1.5857 \mathrm{E}+11$ & $6.3062 \mathrm{E}-12$ & & & \\
\hline No 255 & 255.0932 & $3.100 E+00$ & minute & $1.86 \mathrm{E}+02$ & $2.3777 E+08$ & $4.2057 \mathrm{E}-09$ & & & \\
\hline No 256 & 256.0943 & $2.910 E+00$ & second & $2.91 E+00$ & $1.5139 E+10$ & $6.6057 \mathrm{E}-11$ & & & \\
\hline No 257 & 257.0969 & $2.500 E+01$ & second & $2.50 E+01$ & $1.7553 E+09$ & $5.6972 \mathrm{E}-10$ & & & \\
\hline No 258 & 258.0982 & $1.200 \mathrm{E}-03$ & second & $1.20 \mathrm{E}-03$ & $3.6426 E+13$ & $2.7453 \mathrm{E}-14$ & & & \\
\hline No 259 & 259.1010 & $5.800 E+01$ & minute & $3.48 \mathrm{E}+03$ & $1.2512 \mathrm{E}+07$ & $7.9923 \mathrm{E}-08$ & & & \\
\hline No 260 & 260.1026 & $1.060 \mathrm{E}-01$ & second & $1.06 \mathrm{E}-01$ & $4.0919 E+11$ & $2.4438 \mathrm{E}-12$ & & & \\
\hline No 262 & 262.1075 & $5.000 \mathrm{E}-03$ & second & $5.00 \mathrm{E}-03$ & $8.6085 E+12$ & $1.1616 \mathrm{E}-13$ & & & \\
\hline $\operatorname{Lr} 253$ & 253.0953 & $1.300 E+00$ & second & $1.30 E+00$ & $3.4289 \mathrm{E}+10$ & $2.9164 \mathrm{E}-11$ & & & \\
\hline Lr 254 & 254.0966 & $1.300 \mathrm{E}+01$ & second & $1.30 E+01$ & $3.4153 E+09$ & $2.9280 \mathrm{E}-10$ & & & \\
\hline Lr 255 & 255.0968 & $2.200 E+01$ & second & $2.20 E+01$ & $2.0102 \mathrm{E}+09$ & $4.9745 E-10$ & & & \\
\hline Lr 256 & 256.0988 & $2.800 \mathrm{E}+01$ & second & $2.80 E+01$ & $1.5733 E+09$ & $6.3561 \mathrm{E}-10$ & & & \\
\hline Lr 257 & 257.0996 & $6.460 \mathrm{E}-01$ & second & $6.46 \mathrm{E}-01$ & $6.7927 E+10$ & $1.4722 \mathrm{E}-11$ & & & \\
\hline Lr 258 & 258.1019 & $3.900 E+00$ & second & $3.90 \mathrm{E}+00$ & $1.1208 \mathrm{E}+10$ & $8.9224 \mathrm{E}-11$ & & & \\
\hline $\operatorname{Lr} 259$ & 259.1030 & $6.100 E+00$ & second & $6.10 E+00$ & $7.1380 \mathrm{E}+09$ & $1.4010 \mathrm{E}-10$ & & & \\
\hline Lr 260 & 260.1056 & $1.800 \mathrm{E}+02$ & second & $1.80 E+02$ & $2.4097 \mathrm{E}+08$ & $4.1500 \mathrm{E}-09$ & & & \\
\hline Lr 261 & 261.1069 & $3.900 \mathrm{E}+01$ & minute & $2.34 E+03$ & $1.8465 E+07$ & $5.4157 \mathrm{E}-08$ & & & \\
\hline Lr 262 & 262.1097 & $3.600 \mathrm{E}+00$ & hour & $1.30 E+04$ & $3.3211 \mathrm{E}+06$ & $3.0110 \mathrm{E}-07$ & & & \\
\hline Rf 253 & 253.1007 & $1.800 E+00$ & second & $1.80 \mathrm{E}+00$ & $2.4763 E+10$ & $4.0382 E-11$ & & & \\
\hline Rf 254 & 254.1002 & 5.000 E- 04 & second & $5.00 \mathrm{E}-04$ & $8.8798 \mathrm{E}+13$ & $1.1262 \mathrm{E}-14$ & & & \\
\hline $\operatorname{Rf} 255$ & 255.1015 & $1.500 \mathrm{E}+00$ & second & $1.50 \mathrm{E}+00$ & $2.9483 E+10$ & $3.3918 \mathrm{E}-11$ & & & \\
\hline Rf 256 & 256.1012 & $6.700 \mathrm{E}-03$ & second & $6.70 \mathrm{E}-03$ & $6.5749 \mathrm{E}+12$ & $1.5209 \mathrm{E}-13$ & & & \\
\hline Rf 257 & 257.1031 & $4.700 E+00$ & second & $4.70 E+00$ & $9.3362 \mathrm{E}+09$ & $1.0711 \mathrm{E}-10$ & & & \\
\hline Rf 258 & 258.1036 & $1.200 \mathrm{E}-02$ & second & $1.20 \mathrm{E}-02$ & $3.6425 \mathrm{E}+12$ & $2.7454 \mathrm{E}-13$ & & & \\
\hline Rf 259 & 259.1056 & $3.100 \mathrm{E}+00$ & second & $3.10 E+00$ & $1.4046 \mathrm{E}+10$ & $7.1197 \mathrm{E}-11$ & & & \\
\hline Rf 260 & 260.1064 & $2.010 \mathrm{E}-02$ & second & $2.01 \mathrm{E}-02$ & $2.1579 E+12$ & $4.6342 E-13$ & & & \\
\hline
\end{tabular}

WSRC-TR-99-00344

October, 1999

page 78 


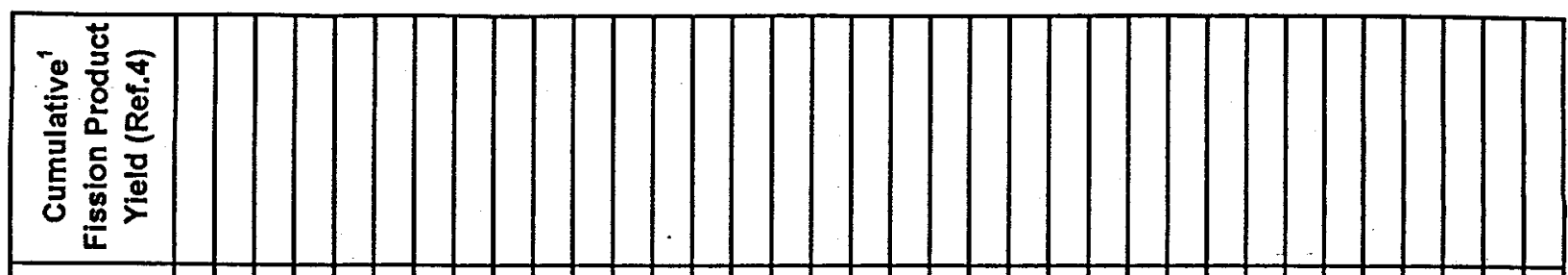

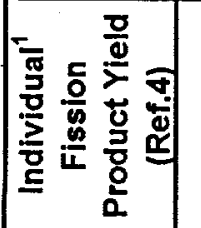

\section{空}

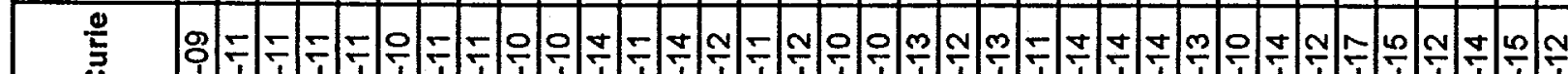

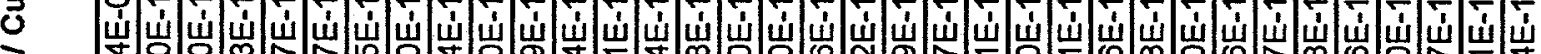

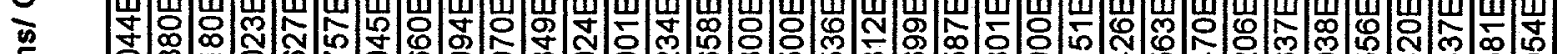

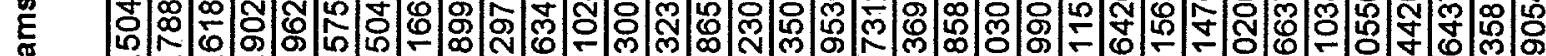

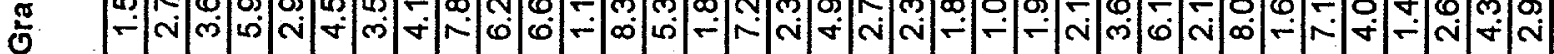

E

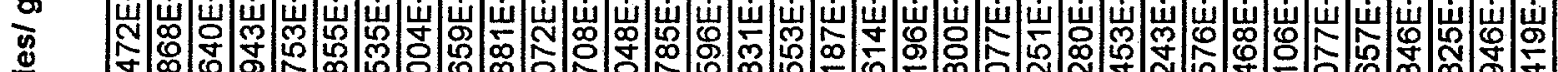
ᄂ

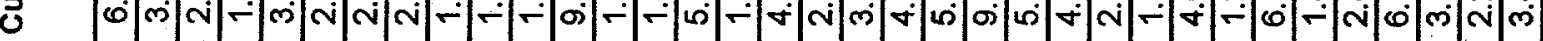

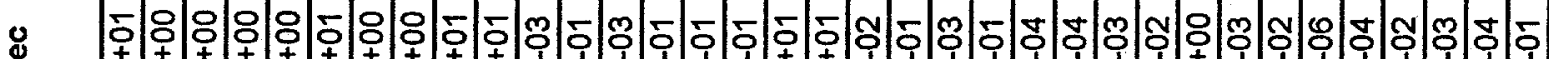

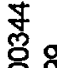
잉

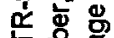
与。 它

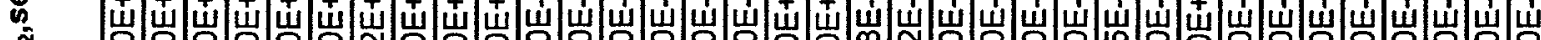

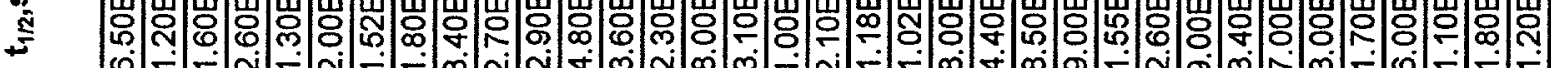

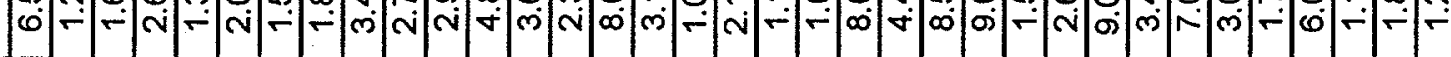

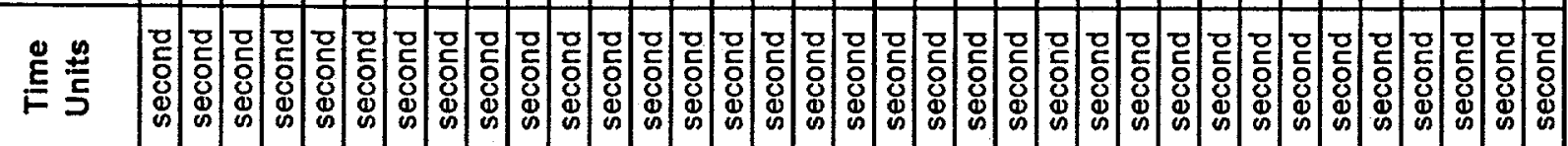

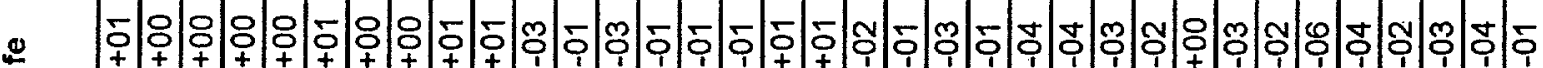

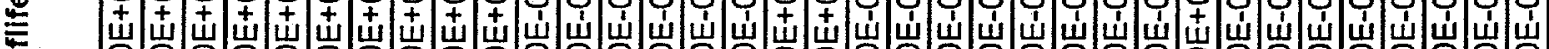

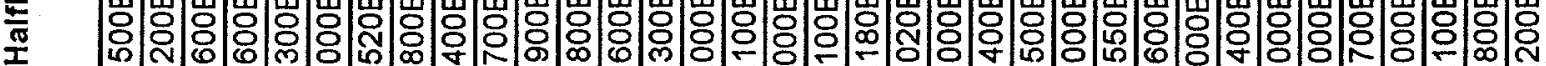

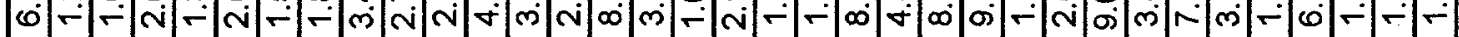

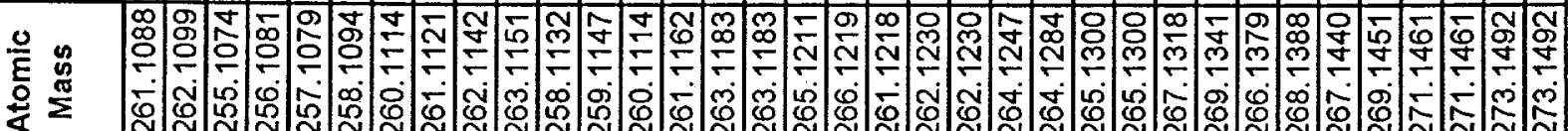

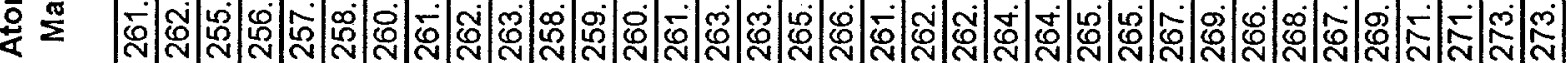

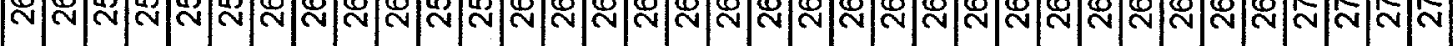

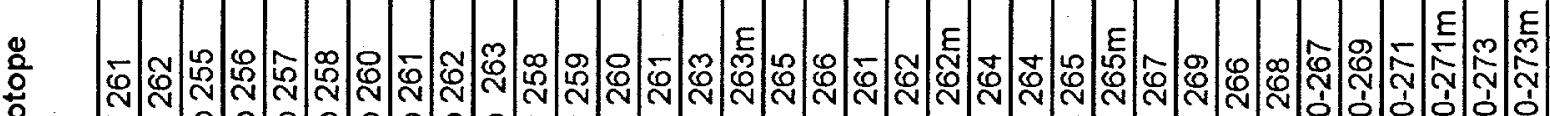

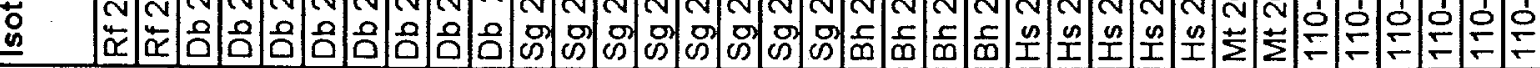


Radionuclide Specific Data

\begin{tabular}{|l|c|c|c|c|c|c|c|c|c|}
\hline Isotope & $\begin{array}{c}\text { Atomic } \\
\text { Mass }\end{array}$ & Halflife & $\begin{array}{c}\text { Time } \\
\text { Units }\end{array}$ & $\mathrm{t}_{1 / 2}, \mathbf{s e c}$ & Curies/gram & Grams/ Curie & $\begin{array}{c}\text { Watts/Ci } \\
\text { (Ref.3) }\end{array}$ & $\begin{array}{c}\text { Individual' } \\
\text { Fission } \\
\text { Product Yield } \\
\text { (Ref.4) }\end{array}$ & $\begin{array}{c}\text { Cumulative } \\
\text { Fission Product } \\
\text { Yield (Ref.4) }\end{array}$ \\
\hline $111-272$ & 272.1535 & $1.500 \mathrm{E}-03$ & second & $1.50 \mathrm{E}-03$ & $2.7636 \mathrm{E}+13$ & $3.6185 \mathrm{E}-14$ & & & \\
\hline $112-277$ & 277.0000 & $2.400 \mathrm{E}-04$ & second & $2.40 \mathrm{E}-04$ & $1.6970 \mathrm{E}+14$ & $5.8927 \mathrm{E}-15$ & & & \\
\hline $114-287$ & 287.0000 & $<0.5$ & second & $<0.5$ & & & & & \\
\hline $114-289$ & 289.0000 & $<0.5$ & second & $<0.5$ & & & & & \\
\hline $116-289$ & 289.0000 & $<0.5$ & second & $<0.5$ & & & & & \\
\hline $118-293$ & 293.0000 & $<0.5$ & second & $<0.5$ & & & & & \\
\hline
\end{tabular}

